\title{
Mecanismos para a Melhoria do Desempenho de Sistemas RFID Passivos
}

\author{
Rafael Perazzo Barbosa Mota
}

\section{TESE APRESENTADA}

$\mathrm{AO}$

Instituto DE MATEMÁtica e Estatística

DA

Universidade DE SÃo PAUlo

PARA

OBTENÇÃO DO TÍTULO

$\mathrm{DE}$

Doutor EM CIÊNCIAS

Programa: Programa de Pós Graduação em Ciência da Computação (PPG-CC)

Orientador: Prof. Dr. Daniel Macêdo Batista

Durante o desenvolvimento desta tese o autor recebeu auxílio financeiro do CNPq São Paulo, Novembro de 2015 


\title{
Mecanismos para a Melhoria do Desempenho de Sistemas RFID Passivos
}

\author{
Esta versão da tese contém as correções e alterações sugeridas \\ pela Comissão Julgadora durante a defesa da versão original do trabalho, \\ realizada em 23/11/2015. Uma cópia da versão original está disponível no \\ Instituto de Matemática e Estatística da Universidade de São Paulo.
}

Comissão Julgadora:

- Prof. Dr. Daniel Macedo Batista (orientador) - IME-USP

- Prof. Dr. Hélio Crestana Guardia - UFSCar

- Prof. Dr. Sérgio Donizetti Zorzo - UFSCar

- Prof. Dr. Gustavo Bittencourt Figueiredo - UFSCar

- Prof. Dr. Luciano Bernardes de Paula - UFSCar 


\section{Dedicatória}

Dedico este trabalho aos meus pais Ocian e Alessandra e à minha esposa Élida. 


\section{Resumo}

MOTA, R. P. B. Mecanismos para a Melhoria do Desempenho de Sistemas RFID Passivos. 2015. 160 f. Tese de Doutorado - Instituto de Matemática e Estatística, Universidade de São Paulo, São Paulo, 2015.

A Identificação por radiofrequência (Radio Frequency Identification - RFID) tem revolucionado a forma de identificar objetos, sendo usada desde aplicações de controle de estoques até o processo automatizado de pagamentos. Sua ampla aceitação e aplicabilidade tem estimulado pesquisadores a criar cada vez mais aplicações.

Um problema chave da RFID são as colisões que ocorrem na identificação por meio dos protocolos de acesso múltiplo. Como, na prática, um leitor precisa identificar várias etiquetas em sua área de cobertura, algumas etiquetas podem responder ao mesmo tempo o que gera colisões e desperdício de recursos. Por este motivo, torna-se de grande valor um estudo abrangente sobre como melhorar a identificação das etiquetas de modo a reduzir o número de colisões. Além disso, aspectos como consumo de energia e tempo necessário para identificação também devem ser levados em consideração, uma vez que a utilização cada vez maior de dispositivos alimentados à bateria tem sido observada na prática.

Esta tese investiga a categoria de protocolos anticolisão denominada Frame Slotted Aloha - FSA, pois é a categoria que possui maior potencial de utilização prática em sistemas RFID. Além disso, as diferentes métricas de análise de desempenho são também analisadas e categorizadas, uma vez que identificou-se que um conjunto de métricas devem ser observadas com o intuito de realizarem-se comparações justas com as propostas da literatura.

Descobriu-se que a maioria das propostas não levam em consideração os aspectos chave de tempo e energia, assim como a característica de ser fácil de implementar e baixa complexidade. Esta tese propõe quatro algoritmos que visam diminuir o consumo de energia e o tempo do processo de identificação das etiquetas mantendo-se as características de baixa complexidade e similaridade com o padrão atual EpcGlobal Classe 1 Geração 2 (C1G2). O primeiro mecanismo visa diminuir a quantidade de respostas desnecessárias em cenários de localização e rastreamento. Os demais consistem em três propostas de algoritmos anticolisão para sistemas RFID. Os dois primeiros diferem na forma como o tamanho inicial de quadro é definido e como as colisões são tratadas, representando evoluções progressivas em direção a um melhor desempenho. O terceiro considera a ocorrência do efeito captura, o que traz a necessidade de mudanças no funcionamento do algoritmo anterior. Resultados de simulação 
mostram que os quatro mecanismos podem melhorar propostas existentes sem aumento de complexidade, resultando consequentemente em diminuição de recursos desperdiçados. Além disso também foram desenvolvidos dois softwares de apoio aos mecanismos propostos: nsRFIDsim e jRFIDsim. O primeiro trata-se de um módulo para o simulador ns-2 que simula um sistema RFID passivo. O segundo implementa uma proposta de benchmark para avaliação de desempenho de algoritmos anticolisão para RFID, visando fornecer para a comunidade científica uma forma padronizada de avaliar este tipo de algoritmo.

Palavras-chave: RFID, Análise de Desempenho, Anticolisão, Consumo de Energia. 


\section{Abstract}

\section{MOTA, R. P. B. Efficient Mechanisms for Performance Improvement in Passive}

RFID Systems. 2015. 160 p. PhD Thesis - Instituto de Matemática e Estatística, Universidade de São Paulo, São Paulo, 2015.

The Radio Frequency Identification - RFID has revolutionized the way objects are identified, being used in several areas, from inventory control applications to automated payment process. Its wide acceptance and applicability has stimulated researchers to create more and more new applications.

One RFID key problem is the collisions on identification by means of multiple access protocols. Since generally, a reader needs to identify multiple tags in its coverage area, some tags tend to reply at the same time which can cause collision and waste of resources. For this reason a comprehensive study on how to improve the identification of tags in order to reduce the number of collisions becomes important. Additionally, issues such as energy consumption and time required for identification must also be taken into consideration, since an increasing use of battery-powered devices has been observed in practice.

This thesis investigates the category of anti-collision protocols called Frame Slotted Aloha - FSA, as it is the category that has the greatest potential for practical use in RFID systems. Further analysis of the different performance metrics are also analyzed and categorized, since it was identified that a set of metrics must be observed in order to carry out up fair comparisons with the proposals found on the literature.

It was found that most of the proposals do not consider the key aspects of time and energy as well as the characteristic of being easy to implement and having low complexity. This thesis proposes four algorithms aimed at reducing the consumption of energy and the time to identify the tags while maintaining the characteristics of low complexity and similarity to the current standard EPCglobal Class 1 Generation 2 (C1G2). The first mechanism aims to decrease the unnecessary answers in locating and tracking scenarios. The other ones consist of three proposals for anti-collision algorithms for RFID systems. They differ in how the initial size is defined and how collisions are resolved, representing progressive evolutions toward a better performance. The third one considers the occurrence of the capture effect, which brings the need for changes in the previous algorithm operation. Simulation results show that the four mechanisms can improve existing proposals without increasing complexity, resulting in consequent energy savings. In addition we have also developed two softwares 
to evaluate the proposed mechanisms: nsRFIDsim and jRFIDsim. The former is a module for the ns-2 simulator that simulates a passive RFID system. The second one implements a proposal for benchmark performance evaluation of anti-collision algorithms for RFID in order to provide to the scientific community a standardized way to assess this type of algorithm.

Keywords: RFID, Performance Evaluation, Anti-collision, Energy Consumption. 


\section{Lista de Figuras}

2.1 O Sistema RFID . . . . . . . . . . . . . . . . . . . . 10

2.2 Etiqueta RFID genérica. . . . . . . . . . . . . . . . . . 11

2.3 Leitor e Etiquetas RFID em dois formatos. . . . . . . . . . . . . . . . . . . . 11

2.4 Leitores RFID em diferentes formatos. . . . . . . . . . . . . . . . . . . . . . 14

2.5 O problema das colisões em sistemas RFID. . . . . . . . . . . . . . . 16

2.6 Taxonomia de protocolos/algoritmos anticolisão para sistemas RFID. . . . . 17

2.7 Exemplo de funcionamento do (D)FSA . . . . . . . . . . . . . . . . . . 19

2.8 Exemplo de efeito captura . . . . . . . . . . . . . . . . 25

3.1 Taxonomia de protocolos/algoritmos FSA para sistemas RFID. . . . . . . . . 28

3.2 Resumo do DFSA-2 . . . . . . . . . . . . . . . . . . . 30

3.3 Diagrama de estados das etiquetas. Adaptado de EPCglobal, GS1 Inc. (2015) 32

3.4 Troca de mensagens para identificar uma única etiqueta. . . . . . . . . . . . 34

3.5 Fluxograma da proposta de Chen (2014). . . . . . . . . . . . . 35

3.6 Fluxograma do FZE (Cui e Zhao, 2010). . . . . . . . . . . . . . . . . . . 39

3.7 Fluxograma da proposta de Tong et al. (2012). . . . . . . . . . . . . . . . 42

3.8 Fluxograma da teoria do paradoxo de aniversários. . . . . . . . . . . . . . . 43

3.9 Fluxograma da proposta de Yao et al. (2014) . . . . . . . . . . . . . . . . 44

3.10 Fluxograma do procedimento de estimação de Cui e Zhao (2009). . . . . . . 46

3.11 Fluxograma da proposta de Cui e Zhao (2009) . . . . . . . . . . . . . . 47

3.12 Fluxograma do Algoritmo BTSA-D. Baseado em Wu et al. (2013). . . . . . 51

3.13 Fluxograma do Algoritmo BTSA-A. Adaptado de Wu et al. (2013). . . . . . 51

3.14 Fluxograma da proposta de He e Wang (2013). . . . . . . . . . . . . . . . 52

3.15 Fluxograma da proposta de Wang et al. (2014). . . . . . . . . . . . . . . 54

4.1 Taxonomia de métricas de avaliação de desempenho. . . . . . . . . . . . . 60

4.2 Tráfego entre leitor e etiquetas de acordo com o tipo de slot. . . . . . . . . . 67

5.1 Máquina de estados das etiquetas para o mecanismo proposto . . . . . . . . . 73

5.2 Cenários modelados . . . . . . . . . . . . . . . . . . . . . . . . 74

5.3 Percentual de perdas x Quantidade de etiquetas para o Cenário 1 . . . . . . . . 77

5.4 Percentual de perdas x Quantidade de etiquetas para o Cenário $2 \ldots$. . . . . 78 
5.5 Quantidade média de Kilobytes transferidos no Cenário 1 . . . . . . . . . . . . 78

5.6 Quantidade média de Kilobytes transferidos no Cenário 2 . . . . . . . . . . . . . 79

5.7 Slots de atraso gerados a partir da utilização de diferentes algoritmos/mecanismos $\quad 80$

5.8 Diagrama de Classes do módulo RFID proposto . . . . . . . . . . . . . . . 82

5.9 Comparação de resultados para eficiência do sistema $\left(S_{e f}\right) \quad \ldots \quad \ldots$. . . . 88

5.10 Comparação de resultados para o total de slots $\left(T_{\text {slots }}\right) \ldots$. . . . . . . . 88

5.11 Comparação de resultados com e sem a aplicação do mecanismo de QoS . . . 89

5.12 Procedimento de estimativa . . . . . . . . . . . . . . . . . . 92

5.13 Melhor valor inicial de Q por número de etiquetas para diferentes valores de i e c 93

5.14 Sobrecarga do procedimento de estimativa para diferentes valores de i e c . . 94

5.15 Proposta NEDFSA . . . . . . . . . . . . . . . . . . 94

5.16 Média de Colisões por Slot . . . . . . . . . . . . . . . . . . . . 96

5.17 Resolução de colisões utilizando diferentes métodos . . . . . . . . . . . . . . 96

5.18 Eficiência do sistema . . . . . . . . . . . . . . . . . . . . . . . . 98

5.19 Total de slots . . . . . . . . . . . . . . . . . . . . . . . . . . . . 99

5.20 Redução de slots comparado ao Algoritmo Q . . . . . . . . . . . . . . . . . 100

5.21 Organização do jRFIDsim. . . . . . . . . . . . . . . . . . . . . 102

5.22 Diagrama de classes do jRFIDsim. . . . . . . . . . . . . . . . 103

5.23 Método DFSA genérico. . . . . . . . . . . . . . . . . . . . 104

5.24 Método de simulação geral. . . . . . . . . . . . . . . . . . . . . 105

5.25 Desempenho do jRFIDsim. . . . . . . . . . . . . . . 107

5.26 Eficiência do Sistema $(\mathrm{C} 1 \mathrm{G} 2)$. . . . . . . . . . . . . . . 108

5.27 Número total de slots utilizados (DBTSA) . . . . . . . . . . . . . . . . 108

5.28 Eficiência do Sistema (Lower Bound) . . . . . . . . . . . . . . . . . . . . . . 109

5.29 Eficiência do Sistema (Schoute) . . . . . . . . . . . . . . . . . . 109

5.30 IDFSA-IcolR: Fluxograma. . . . . . . . . . . . . . . . . . . . 112

5.31 Desempenho do procedimento de estimativa. . . . . . . . . . . . . . . . 117

5.32 Overhead (em slots) do procedimento de estimativa. . . . . . . . . . . 118

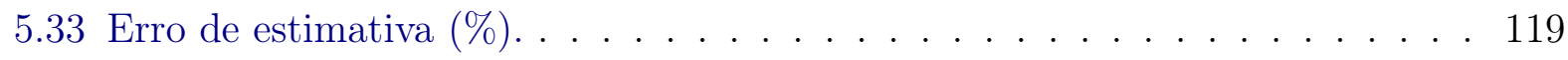

5.34 Eficiência do Sistema. . . . . . . . . . . . . . . . . . . . . . 123

5.35 Número total de slots utilizados. . . . . . . . . . . . . . . . . . . . . . 123

5.36 Tempo total de identificação. . . . . . . . . . . . . . . . . . . 124

5.37 Total de energia desperdiçada devido a colisões e slots vazios. . . . . . . . . 125

5.38 Ganhos na eficiência de uso do canal em relação a outras propostas. . . . . . 126

5.39 Eficiência do Sistema. . . . . . . . . . . . . . . . . . . . . . . . . 134

5.40 Total de slots. . . . . . . . . . . . . . . . . . . . . . . . 134

5.41 Eficiência de uso do canal $(\%) \ldots \ldots \ldots \ldots$. . . . . . . . . . . . . . . . . . . . . . . . . . .

5.42 Quantidade de colisões. . . . . . . . . . . . . . . . . . . . . 135

5.43 Slots desperdiçados. . . . . . . . . . . . . . . . . 136

5.44 Consumo de energia (Joules) . . . . . . . . . . . . . . 136 
5.45 Tempo total de identificação. . . . . . . . . . . . . . . . . . . . . 137 


\section{Lista de Tabelas}

2.1 Classificação quanto à fonte de energia (Klair et al., 2010) . . . . . . . . . 12

2.2 Classificação quanto à faixa de frequência de operação (Hanada e Kudou, 2013) 13

2.3 Protocolos para as diferentes frequências de operação de RFID . . . . . . . . 15

2.4 Descrição de acrônimos. . . . . . . . . . . . . . . . . . . . . . . 26

3.1 Tabela comparativa entre várias propostas da literatura. . . . . . . . . . . 58

4.1 Tempo de identificação (em ms) para diferentes tipos de slots. . . . . . . . . . 62

4.2 Parâmetros típicos do protocolo $\mathrm{C} 1 \mathrm{G} 2 \ldots \ldots$. . . . . . . . . . . . . 63

4.3 Métricas de avaliação de desempenho. . . . . . . . . . . . . . . . . . . . . 69

5.1 Redução do número de pacotes gerados, comparados com o Algoritmo Q . 79

5.2 Arquivos (M)odificados $/(\mathrm{C})$ riados . . . . . . . . . . . . . . . . 82

5.3 Formato do arquivo de trace . . . . . . . . . . . . . . . . . . . 84

5.4 Parâmetros do primeiro conjunto de simulações . . . . . . . . . . . . . . . . 86

5.5 Parâmetros do segundo conjunto de simulações . . . . . . . . . . . . . . . . . 87

5.6 Exemplo de tabela da métrica Eficiência do Sistema. . . . . . . . . . . . . 111

5.7 Probabilidades de efeito captura baseado na quantidade de etiquetas envolvidas na colisão.133

7.1 Desafios em aberto . . . . . . . . . . . . . . . . . . . 144 


\section{Lista de Algoritmos}

1 Algoritmo FSA: Operação do Leitor . . . . . . . . . . . . . . . . . . . 21

2 Algoritmo Q: Operação do Leitor . . . . . . . . . . . . . . . . . . . 30

3 Algoritmo Q: Operação das etiquetas . . . . . . . . . . . . . . . . . 31

4 Função de estimação de Eom-Lee: Operação do Leitor . . . . . . . . . . . . . . 38

$5 \quad$ Algoritmo BTSA-D: Operação do Leitor. Adaptado de (Wu et al., 2013) . . 49

6 Mecanismo implementado nas etiquetas para garantia de QoS . . . . . . 73

7 Algoritmo NEDFSA . . . . . . . . . . . . . . . . . 95

8 Função de resolução de colisões DFSA - CH-DFSA . . . . . . . . . . . . . . . 97

9 IDFSA-IcolR . . . . . . . . . . . . . . . . . . . . . . . . . . . . 113

10 Função de tratamento de slot de colisão. . . . . . . . . . . . . . . . . . . . 114

11 IDFSA-IcolR-EC: Operação principal do leitor . . . . . . . . . . . . . . . . . 129

12 IDFSA-IcolR-EC: Função de tratamento de slot de colisão. . . . . . . . . . . . 130

13 IDFSA-IcolR-EC: Operação da etiqueta . . . . . . . . . . . . . . . . . . . 130 


\section{Sumário}

Lista de Figuras $\quad$ vii

Lista de Tabelas $\quad$ xi

Lista de Algoritmos $\quad$ xiii

Lista de Abreviaturas $\quad$ xix

1 Introdução $\quad 1$

1.1 Motivação . . . . . . . . . . . . . . . . . . . . . . 3

1.2 Descrição do Problema . . . . . . . . . . . . . . . . . . . . . 4

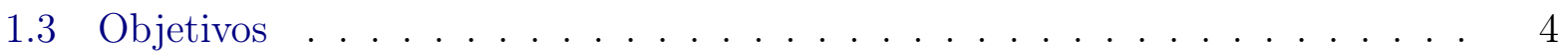

1.4 Contribuições . . . . . . . . . . . . . . . . . . . . . . . . 4

1.4.1 Publicações . . . . . . . . . . . . . . . . 6

1.5 Organização da Tese . . . . . . . . . . . . . . . . . . . . 8

2 Contextualização $\quad 9$

2.1 Identificação por Radiofrequência - RFID . . . . . . . . . . . . . . . . 9

2.2 Protocolos de Acesso ao meio . . . . . . . . . . . . . . . . . . . . . . 15

2.3 Resolução de Colisões em Sistemas RFID Passivos . . . . . . . . . . . . . . . 16

2.4 Probabilidade e Distribuição Binomial . . . . . . . . . . . . . . . . . 20

2.5 Simuladores RFID . . . . . . . . . . . . . . . . . . . . 23

2.6 Efeito Captura . . . . . . . . . . . . . . . . . . . . . . 25

2.7 Notações e acrônimos . . . . . . . . . . . . . . . . . . . . . . . . . 25

3 Protocolos anticolisão para sistemas RFID passivos $\quad 27$

3.1 Taxonomia . . . . . . . . . . . . . . . . . . . . . . . 28

3.2 DFSA baseado em potência de $2($ DFSA-2) . . . . . . . . . . . . . . 28

3.2.1 Algoritmo Q - C1G2 . . . . . . . . . . . . . . 29

3.2.2 Wang et al. (2009) . . . . . . . . . . . . . . . 33

3.2 .3 Chen (2014) . . . . . . . . . . . . . . . . 33

3.2.4 Chen (2015) . . . . . . . . . . . . . . . 35

3.3 DFSA Puro com estimação de backlog (PDFSA-b) . . . . . . . . . . . 36 
3.3.1 Zhu e Yum (2010) . . . . . . . . . . . . . . 38

3.3 .2 Cui e Zhao (2010) . . . . . . . . . . . . . . . . . . 39

3.3 .3 Tong et al. (2012) . . . . . . . . . . . . . . 40

3.3.4 Shakiba et al. (2014) . . . . . . . . . . . . . . 41

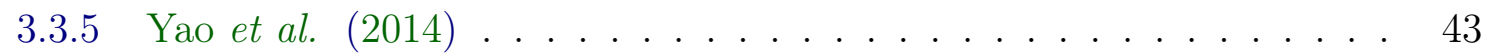

3.3.6 Lin et al. (2015) . . . . . . . . . . . . . . . . . 45

3.4 Binary Tree Slotted Aloha . . . . . . . . . . . . . . . . 45

3.4.1 Cui e Zhao (2009) . . . . . . . . . . . . . . 46

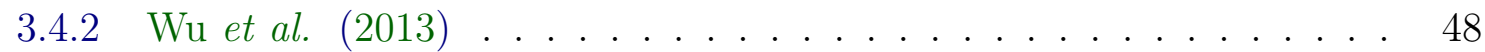

3.5 Híbridos . . . . . . . . . . . . . . . . . . . . . 51

3.5.1 He e Wang (2013) . . . . . . . . . . . . . . . 52

3.5.2 Wang et al. (2014) . . . . . . . . . . . . . . . 53

3.5.3 Xu e Chen (2015) . . . . . . . . . . . . . . 55

3.6 Propostas que lidam com efeito captura . . . . . . . . . . . . . . 56

3.7 Resumo . . . . . . . . . . . . . . . . . . . . . 57

4 Métricas de Avaliação de Desempenho $\quad 59$

4.1 Slots . . . . . . . . . . . . . . . . . . . . . 60

4.2 Tempo de identificação . . . . . . . . . . . . . . . . . . . 61

4.3 Energia . . . . . . . . . . . . . . . . . . . . 64

4.4 Tráfego . . . . . . . . . . . . . . . . . . . . . 65

4.5 Precisão . . . . . . . . . . . . . . . . . . . . . . 67

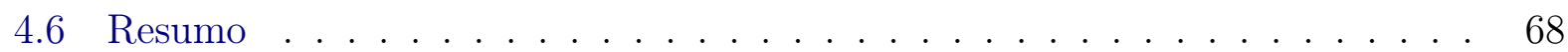

$\begin{array}{lll}5 & \text { Mecanismos propostos } & 71\end{array}$

5.1 Um Mecanismo para redução de respostas repetitivas em RFID . . . . . . . 72

5.1 .1 Mecanismo proposto . . . . . . . . . . . . . . . . . 72

5.1 .2 Cenários e experimentos . . . . . . . . . . . . . . . . . . . 74

5.1 .3 Resultados e discussão . . . . . . . . . . . . . . . . . . 76

5.1 .4 Conclusões parciais . . . . . . . . . . . . . . . . . 79

5.2 Um Módulo do ns-2 para simular Sistemas RFID Passivos . . . . . . . . . . 81

5.2.1 Detalhes do módulo RFID para ns-2 . . . . . . . . . . . . . . . . 81

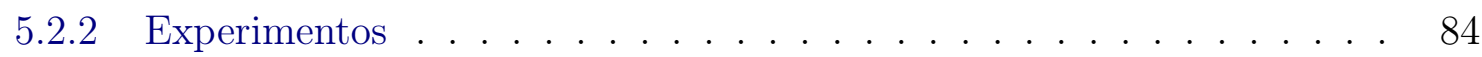

5.2 .3 Resultados e Validação . . . . . . . . . . . . . . . . . . . . . . . . 87

5.2 .4 Conclusões parciais . . . . . . . . . . . . . . . . . . . . 89

5.3 Um algoritmo DFSA estimado: NEDFSA . . . . . . . . . . . . . . . 91

5.3.1 Algoritmo proposto . . . . . . . . . . . . . . 91

5.3 .2 Resultados . . . . . . . . . . . . . . . . . . . . . . . . . . . . . . . 98

5.3 .3 Conclusões parciais . . . . . . . . . . . . . . . . . . . . . 100

5.4 Benchmark para avaliação de desempenho de sistemas RFID . . . . . . . . . 100 
5.4.1 Visão geral do jRFIDsim . . . . . . . . . . . . . . . . . . . . 102

5.4 .2 Desempenho do jRFIDsim . . . . . . . . . . . . . . . 106

5.4 .3 Validação do jRFIDsim . . . . . . . . . . . . . . . . . 106

5.4.4 Os testes de benchmark do jRFIDsim . . . . . . . . . . . . . . . . 110

5.4.5 Conclusões parciais . . . . . . . . . . . . . . . . . . . . 110

5.5 Um algoritmo eficiente em energia e tempo para RFID . . . . . . . . . . . 111

5.5.1 Análise de desempenho . . . . . . . . . . . . . . . . . . . 115

5.5.2 Parâmetros de simulação . . . . . . . . . . . . . . . . . . . . . 119

5.5.3 Simulações . . . . . . . . . . . . . . . . . . . . . 120

5.5.4 Resultados de simulação . . . . . . . . . . . . . . . . . . . 122

5.5.5 Conclusões parciais . . . . . . . . . . . . . . . 126

5.6 Um algoritmo anticolisão para sistemas RFID sob o efeito captura . . . . . . 127

5.6 .1 O IDFSA-IcolR-EC . . . . . . . . . . . . . . . . . . . . . . 128

5.6.2 Parâmetros de simulação . . . . . . . . . . . . . . . . . . . 131

5.6 .3 Simulações . . . . . . . . . . . . . . . . . . . . . . . . . . 132

5.6 .4 Resultados . . . . . . . . . . . . . . . . . . . 133

5.6 .5 Conclusões parciais . . . . . . . . . . . . . . . . . 137

6 Conclusões 139

7 Trabalhos Futuros $\quad 143$

7.1 Mobilidade. . . . . . . . . . . . . . . . . . . 143

7.2 Múltiplos leitores e ambientes densos . . . . . . . . . . . . . . . . 143

7.3 Interferência . . . . . . . . . . . . . . . . . . . . . . . . . . 144

$\begin{array}{lr}\text { A Exemplos } & 147\end{array}$

$\begin{array}{ll}\text { Referências Bibliográficas } & 151\end{array}$ 
XViii SUMÁRIO 


\title{
Lista de Abreviaturas
}

\author{
RFID Radio Frequency Identification - Identificação por radiofrequência \\ RSSF Redes de sensores sem fio \\ C1G2 Tipo de etiqueta Classe 1 Geração 2 \\ DFSA Dynamic Frame Slotted Aloha \\ UHF Ultra High Frequency \\ GPS Global Positioning System - Sistema de Posicionamento Global \\ WiMax Worldwide Interoperability for Microwave Access \\ MANET Mobile Ad-hoc Network
}




\section{Capítulo 1}

\section{Introdução}

A Identificação por Radiofrequência (RFID - Radio Frequency Identification) é uma tecnologia de identificação de objetos mais avançada que a tecnologia de código de barras tradicional. A RFID usa um campo magnético ou eletromagnético para identificação de objetos que não requer a necessidade de linha de visada para comunicação entre os objetos. Em contraste, os códigos de barras fazem uma leitura óptica e requerem linha de visada. Outra típica vantagem da RFID é a habilidade de identificação de vários objetos ao mesmo tempo (Finkenzeller et al., 2010). As etiquetas RFID são entidades que precisam ser fixadas nos objetos para permitir a identificação. Elas estão disponíveis em diferentes formatos, configurações e tamanhos. Além disso, os dados armazenados nas etiquetas podem ser alterados por um leitor (entidade que realiza as identificações), fato este impossível de ser realizado em códigos de barras. Estes importantes recursos, aliados com a redução de custos de implementação, vem acelerando a adoção da tecnologia em variados tipos de negócios. Aplicações do RFID incluem entre outros a prevenção de roubos de automóveis e mercadorias, a passagem automática em pedágios, o controle da cadeia de suprimentos, o controle de estoques, cuidados em saúde, localização e rastreamento, apenas para citar alguns (Dobkin, 2012).

Desde o início dos anos dois mil, a RFID vem ganhando destaque publicitário devido à sua adoção por grandes grupos como WalMart, Tesco e o Departamento de Defesa americano - DoD (Want, 2006). Estas organizações sustentam que a tecnologia RFID foi capaz de reduzir drasticamente os custos, sobrecarga de trabalho e perdas de produtos. De acordo com a IdTech, o valor movimentado pelo mercado de RFID pode atingir 27, 21 bilhões de 
dólares americanos em 2024 (Das e Harrop, 2014). Apenas no ano de 2014, de acordo com Das (2014), o mercado de etiquetas RFID passivas cresceu de 1,12 bilhões para 6, 9 bilhões de etiquetas consumidas. No mercado de varejo tem-se observado um crescimento anual de $39 \%$ nas vendas de leitores, etiquetas e aplicações, prevendo-se um crescimento de 738 milhões para 5, 409 bilhões de dólares movimentados (Swedberg, 2015).

Apesar de estar em estágio maduro de desenvolvimento, a tecnologia RFID ainda não é a principal forma de identificar objetos. Sistemas de código de barras e Código QR ainda são amplamente utilizadas, dado o baixíssimo custo dos mesmos (Campbell, 2011). Apesar da principal vantagem relacionada aos custos, as mesmas possuem como desvantagem a necessidade de linha de visada entre o objeto a ser identificado e o leitor e o requisito de que cada objeto precisa ser identificado um de cada vez. Muitas vezes a leitura é impossibilitada por pequenas falhas na impressão do código ou no posicionamento do leitor, o que pode gerar atrasos na identificação. Estes problemas não ocorrem na utilização de RFID (Finkenzeller et al., 2010), uma vez que não existe a necessidade de linha de visada, e problemas de leitura são raros. Adicionalmente os identificadores dos objetos podem ser lidos com velocidade muito superior a tecnologias como código de barras ou QrCode, pois segundo Namboodiri et al. (2012), sistemas RFID passivos podem identificar cerca de 600 objetos por segundo, sem a necessidade de fazer a leitura individual de cada um dos produtos. Apesar do custo superior em relação aos códigos de barra e QR, este cenário vem mudando rapidamente com a diminuição dos custos de produção das etiquetas (Waktola , 2015)

Um típico sistema RFID consiste de um leitor, uma ou mais etiquetas e uma aplicação para processamento dos dados (Klair et al., 2010). O leitor consiste de um dispositivo rico em recursos, com memória ampla e poder computacional adequado. O mesmo possui quatro funções principais: energizar as etiquetas passivas, gerar o sinal de portadora, difundir os pacotes e decodificar o sinal modulado. Para identificar as etiquetas o leitor energiza-as através da emissão de um campo de sinal eletromagnético que também contém o sinal de portadora a ser utilizado pelas etiquetas para enviar seus identificadores (IDs). O leitor decodifica as respostas recebidas para obter os IDs das etiquetas. Os IDs são enviados para um sistema computacional que será responsável por processar os dados recebidos de acordo 
com os requisitos das aplicações utilizadas. Quando um leitor precisa interagir com mais de uma etiqueta ao mesmo tempo, precisa-se utilizar algum protocolo ou algoritmo que lide com o múltiplo acesso, uma vez que as etiquetas respondem ao mesmo tempo às requisições dos leitores. Estes são chamados de algoritmos anticolisão (Dobkin, 2012; Finkenzeller et al., 2010).

Sistemas RFID tradicionais não foram projetados originalmente para operar com milhões e bilhões de nós, o que tende a ocorrer com a implementação da tecnologia em cenários como controle de estoques ou bibliotecas. Em RFID os algoritmos e protocolos anticolisão desempenham papel fundamental para garantir um desempenho adequado e desejável das aplicações (Wu et al., 2013). Apesar de várias propostas de mecanismos anticolisão tenham sido apresentadas ao longo dos anos, esta tese identificou que aspectos de consumo de energia e tempo não vem sendo adequadamente exploradas. Além disso a investigação de métodos para melhoramento do tamanho inicial de quadro também carece de maior exploração.

\subsection{Motivação}

Um problema chave que afeta o desempenho de sistemas RFID é o processo de identificação múltipla de um conjunto de etiquetas. Os leitores precisam lidar de forma eficiente com as colisões que ocorrem durante o processo de identificação, pois são elas as principais responsáveis pelo aumento do consumo de recursos e pela degradação do desempenho de identificação (Klair et al., 2009).

Até o momento da escrita desta tese, a maioria dos algoritmos anticolisão propostos exploram como melhorar a eficiência de identificação através do ajuste de cada um dos quadros gerados durante o processo (Chen, 2015). Diferentes abordagens com diferentes níveis de complexidade vem sendo exaustivamente investigados. A carência de trabalhos que visem a diminuição do consumo energético especificamente, motivou a exploração de uma abordagem que inicialmente mostra-se promissora: ajustar corretamente o tamanho inicial de quadro e através da análise da teoria do acesso aleatório, diminuir a quantidade de colisões de forma que as mesmas sejam menos prováveis, resultando em diminuição do tempo de identificação e consequentemente do consumo energético. 


\subsection{Descrição do Problema}

Esta tese analisa e propõe mecanismos eficientes para a identificação de etiquetas RFID de forma que o consumo energético e de tempo sejam reduzidos sem que haja a necessidade de aumentar a complexidade de implementação dos leitores atuais. Especificamente, estudamse os protocolos que tratam as colisões (Klair et al., 2010). Como as colisões resultam de respostas simultâneas de mais de uma etiqueta ao mesmo tempo (Hakeem et al., 2014), as mesmas causam o aumento dos atrasos de identificação, aumentando também o consumo de energia dos leitores.

\subsection{Objetivos}

Geral: Desenvolver mecanismos que melhorem o desempenho de sistemas RFID em relação a diferentes métricas.

\section{Específicos:}

- Aumentar o desempenho dos sistemas RFID sem aumento da complexidade do leitor;

- Diminuir o consumo de energia e de tempo de identificação;

- Propor uma padronização para avaliação de desempenho de protocolos/algoritmos anticolisão;

- Propor uma forma precisa de ajuste de tamanho inicial de quadro, permitindo o melhor aproveitamento dos recursos gastos durante o processo de identificação;

- Propor uma forma de lidar com o efeito captura, que é um fenômeno comum em redes sem fio que ocasiona uma incorreta identificação de sucesso em uma etiqueta.

\subsection{Contribuições}

Esta tese contribui para o avanço do estado da arte conforme listado a seguir:

- 1. Uma revisão bibliográfica (survey) de algoritmos anticolisão baseados em FSA. A ausência de trabalhos que abordem especificamente a categoria Frame 
Slotted Aloha - FSA motivou este estudo, uma vez que a categoria FSA possui o maior potencial de utilização prática futura. Apesar disso, alguns surveys relacionados e com abordagem mais generalista foram apresentados por Klair et al. (2010), Shih et al. (2006), Ullah et al. (2012) e Zhu e Yum (2010). A metodologia utilizada nesta tese baseia-se primeiramente na identificação das características chave de cada proposta e posteriormente na identificação da metodologia de avaliação do desempenho, como métricas utilizadas, cenários e softwares.

- 2. Uma revisão bibliográfica de métricas de avaliação de desempenho para algoritmos anticolisão de sistemas RFID. Após a análise e o estudo das diversas propostas da literatura, identificou-se que diferentes métricas são utilizadas para a apresentação da análise de desempenho. Propôs-se uma taxonomia e detalhou-se em profundidade como são calculadas cada uma destas métricas.

- 3. Um módulo RFID para o simulador de eventos discretos ns-2. Com o crescente aumento da relevância da Internet das Coisas, e devido à característica de heterogeneidade da mesma, desenvolveu-se um simulador da tecnologia RFID para avaliação de sistemas RFID. O módulo foi utilizado durante o desenvolvimento desta tese e pode ajudar pesquisadores da área a economizar tempo e padronizar a simulação de novas propostas baseadas na tecnologia RFID.

\section{- 4. Uma proposta de benchmark para avaliação de algoritmos anticolisão para} RFID. A ausência de padronização sobre como avaliar propostas de novos protocolos anticolisão para RFID motivou o desenvolvimento deste framework. Observou-se que diferentes autores utilizam as métricas mais convenientes. No entanto, este fato dificulta a correta comparação entre os métodos. Esta contribuição visa portanto ajudar a comunidade científica especializada a padronizar as formas de avaliação de desempenho.

- 5. Um mecanismo para redução de perda de pacotes para cenários de localização e rastreamento. O funcionamento dos leitores em situações e cenários de localização e rastreamento pode aumentar a perda de pacotes e aumentar o consumo 
energético. Pensando em reduzir este tipo de situação, foi proposto um mecanismo para lidar com o problema.

- 6. Dois mecanismos anticolisão para sistemas RFID: NEDFSA e IDFSAIcolR. Através da abordagem de melhor dimensionar o tamanho inicial de quadro e resolver as colisões no momento em que elas ocorrem, foram propostas duas soluções. A primeira envolve integrar técnicas baseadas nas propostas de Cui e Zhao (2009) e Wu et al. (2013). A segunda faz melhoramentos na estimativa do tamanho inicial de quadro aliada à análise da teoria do acesso aleatório para otimizar a resolução de colisões.

- 7. Um mecanismo anticolisão para funcionamento do ambiente sob influência do efeito captura: IDFSA-IcolR-EC. Baseado nas probabilidades de ocorrência do efeito captura, estendeu-se um dos algoritmos do item anterior para funcionar também nos ambientes de efeito captura, ou seja, ambientes mais próximos das situações reais.

\subsubsection{Publicações}

Até o momento da escrita desta tese, os seguintes artigos foram publicados:

- (Mota e Batista, 2013) Rafael Perazzo Barbosa Mota e Daniel Batista. Um Mecanismo para Garantia de QoS na Internet das Coisas com RFID. Em Proceedings of the SBRC 2013 (Simpósio Brasileiro de Redes de Computadores e Sistemas Distribuídos). Brasília, Brazil;

- (Perazzo Barbosa Mota e Batista, 2013) Perazzo Barbosa Mota, R.; Batista, D.M., An Ns-2 Module for Simulating Passive RFID Systems, High Performance Computing and Communications \& 2013 IEEE International Conference on Embedded and Ubiquitous Computing (HPCC/EUC), 2013 IEEE 10th International Conference on , vol., no., pp.2263,2270, 13-15 Nov. 2013 doi: 10.1109/HPCC.and.EUC.2013.325;

- (Perazzo Barbosa Mota e Batista, 2013) Perazzo Barbosa Mota, R.; Batista, D.M., A RFID QoS Mechanism for IoT Tracking Applications, Wireless and Pervasive Compu- 
ting (ISWPC), 2013 International Symposium on , vol., no., pp.1,4, 20-22 Nov. 2013 doi: 10.1109/ISWPC.2013.6707429;

- (Mota e Batista, 2014) Rafael Perazzo Barbosa Mota and Daniel M. Batista. 2014. A Dynamic Frame Slotted ALOHA Anti-collision Algorithm for the Internet of Things. In Proceedings of the 29th Annual ACM Symposium on Applied Computing (SAC '14). ACM, New York, NY, USA, 686-691. DOI=10.1145/2554850.2555003;

- (Mota, 2014) Rafael Perazzo Barbosa Mota. 2014. Student Research Abstract: Mechanisms to Ensure Quality of Service for the Internet of Things. In Proceedings of the 29th Annual ACM Symposium on Applied Computing (SAC '14). ACM, New York, NY, USA, 713-714. DOI=10.1145/2554850.2555189. (2a Colocação na premiação do Student Research Abstract).

- (Mota, 2015b) Perazzo Barbosa Mota, R.; Batista, D.M., An Easy to Implement and Energy-saving Anti-Collision Algorithm for RFID Systems, Application of Information and Communication Technologies (AICT), 2015 IEEE 9th International Conference on , vol., no., pp.367-372, 14-16 Oct. 2015. doi: 10.1109/ICAICT.2015.7338581.

- (Mota, 2015a) Perazzo Barbosa Mota, R.; Batista, D.M., Simulator and Benchmark for RFID Anti-Collision Evaluation, Application of Information and Communication Technologies (AICT), 2015 IEEE 9th International Conference on , vol., no., pp.614618, 14-16 Oct. 2015. doi: 10.1109/ICAICT.2015.7338634.

Os seguintes artigos foram gerados e estão em fase de submissão:

- RFID Anti-Collision Performance Metrics: A Survey. Computer Communications;

- RFID FSA anti-collision protocols: A Survey. Computer Communications;

- A New Energy Aware Aloha-based Anti-Collision Algorithm for RFID Systems. Plos One. Versão extendida;

- A Benchmark Software for RFID Anti-collision Algorithms. SoftwareX. Versão estendida. 
- IDFSA-IcolR-EC: A new anti-collision algorithm under capture effect. Communication Letters.

\subsection{Organização da Tese}

O restante desta tese está organizado da seguinte forma:

- Capítulo 2. Esse capítulo apresenta a contextualização sobre as características do sistema RFID e como a identificação das etiquetas é realizada.

- Capítulo 3. Esse capítulo apresenta uma revisão bibliográfica sobre as propostas de protocolos anticolisão disponíveis na literatura.

- Capítulo 4. Esse capítulo apresenta as principais categorias e métricas de análise de desempenho de algoritmos anticolisão para sistemas RFID.

- Capítulo 5. Esse capítulo apresenta os resultados obtidos ao longo do desenvolvimento desta tese com os novos mecanismos propostos e softwares desenvolvidos.

- Capítulo 6. Esse capítulo apresenta as conclusões desta tese.

- Capítulo 7. Esse capítulo apresenta os desafios em aberto e possibilidades de trabalhos futuros.

- Apêndice A. Esse apêndice apresenta exemplos práticos de aplicações de conceitos abordados ao longo desta tese. 


\section{Capítulo 2}

\section{Contextualização}

Este capítulo está organizado como a seguir: a seção 2.1 apresenta os conceitos fundamentais da tecnologia RFID, seguida pelas seções 2.2 e 2.3 que tratam dos conceitos de acesso ao meio e como o mesmo é abordado em sistemas RFID. As definições da teoria da probabilidade, que são amplamente estudadas e utilizadas em RFID, são apresentadas na seção 2.4. Finalmente, a seção 2.5 aborda a importância que os simuladores de rede possuem na pesquisa em protocolos anticolisão.

\subsection{Identificação por Radiofrequência - RFID}

A RFID é uma tecnologia voltada para a rápida identificação de objetos, embora nada impeça sua utilização para a identificação de seres vivos (Dobkin, 2012). RFID é utilizada principalmente na identificação automática de objetos, coletando informações sobre os mesmos e transferindo para uma aplicação central, podendo esta informação ser manipulada e transformada em inteligência de negócios (Wang et al., 2012). Apesar de ter se popularizado no começo dos anos 2000, o conceito por trás da RFID foi concebido nos anos 1940. Seu desenvolvimento remonta à época da segunda guerra mundial, no sistema de identificação de Amigos ou Inimigos (Friend or Foe) (Finkenzeller et al., 2010). A ideia nesse sistema era permitir, por exemplo, a identificação de aeronaves, determinando se elas eram amigas. O processo de consulta era realizado através de sinais modulados em frequências bem definidas e eram esperadas respostas padronizadas. Para isso as aeronaves amigas eram equipadas 
com transponders que tinham capacidade de interpretar os sinais recebidos e responder com seus identificadores. Caso as respostas não fossem as esperadas ou se não houvesse respostas, concluía-se que as aeronaves não eram amigas, embora isso não significasse que fossem inimigas (Violino, 2005). A tecnologia é utilizada tanto para uma simples aplicação de identificação quanto para aplicações complexas de rastreamento e localização (Wu et al., 2013).

A tecnologia RFID é utilizada para a construção de sistemas RFID. Em geral, sistemas RFID são formados por três componentes principais (Finkenzeller et al., 2010) listados a seguir e ilustrados na Figura 2.1.

- Etiqueta

- Leitor

- Aplicação

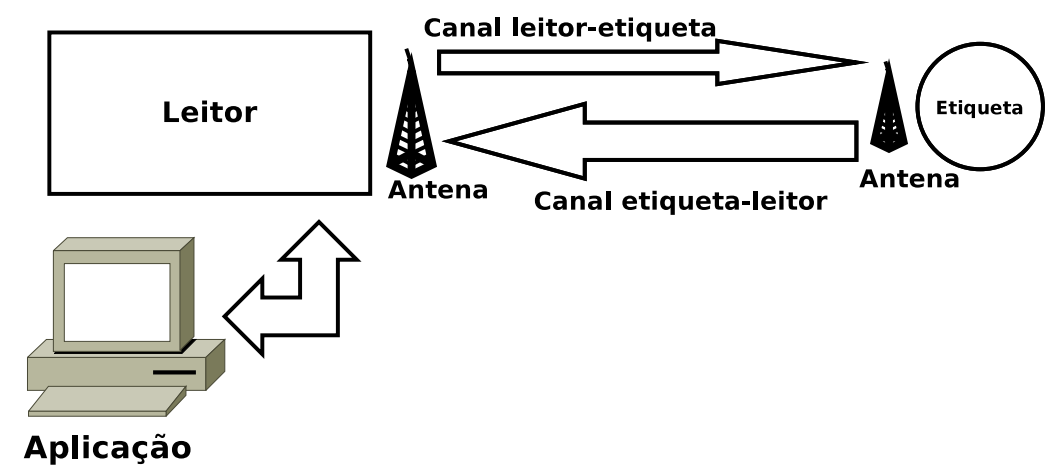

Figura 2.1: O Sistema RFID

As etiquetas são afixadas em cada elemento que será identificado no sistema. Elas estão disponíveis nos mais diversos formatos, tamanhos e materiais, dependendo do contexto da aplicação em que serão utilizadas. Basicamente são formadas por três partes principais: um microchip; uma antena para comunicação; e uma pequena memória (Finkenzeller et al. , 2010). A Figura $2.2^{1}$ apresenta o modelo genérico de etiqueta descrita anteriormente e a foto da Figura $2.3^{2}$ mostra um exemplo de duas etiquetas e um leitor. Pode-se notar que a etiqueta da esquerda (1) (Figura 2.3) apresenta formato de cartão, muito similar a um cartão de crédito convencional. A etiqueta abaixo da régua (3) é do tamanho de uma

\footnotetext{
${ }^{1}$ Figura extraída de http://www2.ministries-online.org/biometrics/rfidchip2.html

${ }^{2}$ Fotos extraídas do acervo pessoal do autor
} 
moeda, no formato de um pequeno chaveiro. Já o leitor é similar a uma unidade de memória usb (pendrive - Figura 2.3).

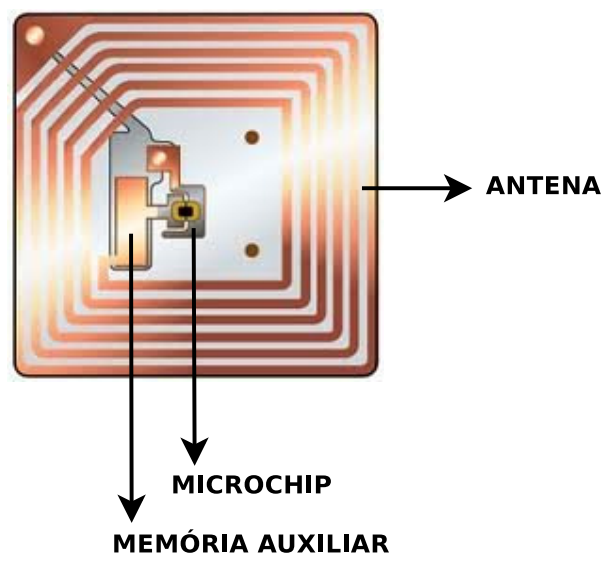

Figura 2.2: Etiqueta RFID genérica.

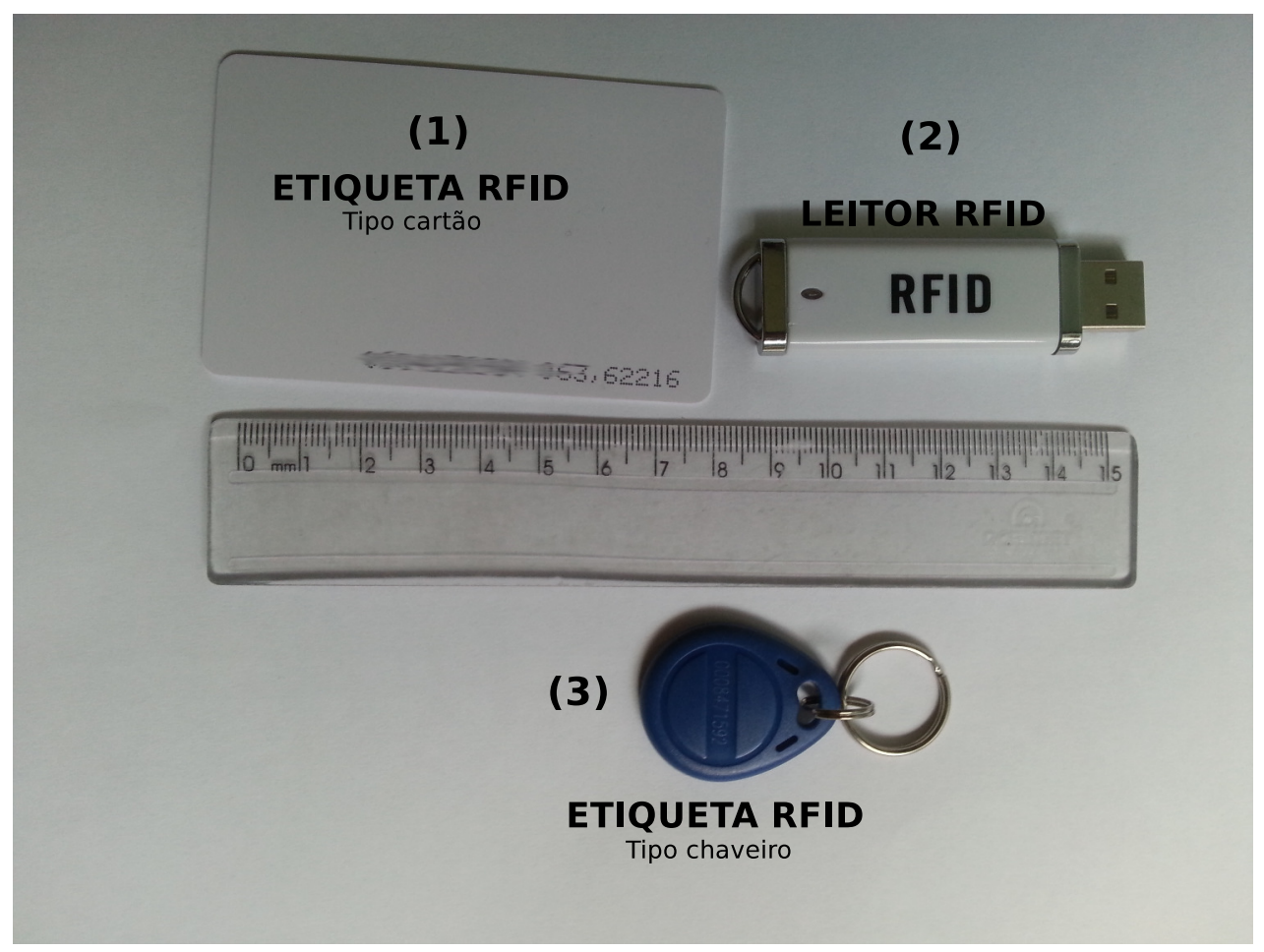

Figura 2.3: Leitor e Etiquetas RFID em dois formatos.

As etiquetas RFID podem ser classificadas de duas formas principais: quanto ao tipo de energia; e quanto à frequência de operação, conforme detalhado nas Tabelas 2.1 e 2.2 (Finkenzeller et al., 2010).

Etiquetas passivas são as mais utilizadas devido ao seu baixo custo de fabricação, quando comparadas com as demais. Enquanto etiquetas passivas podem custar cerca de dez centavos 
Tabela 2.1: Classificação quanto à fonte de energia (Klair et al., 2010)

\begin{tabular}{cl}
\hline Tipo de etiqueta & Características \\
\hline Passivas & $\begin{array}{l}\text { Não possuem fonte de energia própria, sendo energizadas } \\
\text { a partir do sinal do leitor. }\end{array}$ \\
Semi-Passivas & $\begin{array}{l}\text { Possuem fonte própria de energia, mas não podem ini- } \\
\text { ciar uma comunicação. }\end{array}$ \\
Ativas & $\begin{array}{l}\text { Possuem fonte própria de energia sendo possível também } \\
\text { iniciar a comunicação. }\end{array}$ \\
\hline
\end{tabular}

de dólar, uma etiqueta ativa pode chegar a cem dólares dependendo de suas características ${ }^{3}$. Por não possuir fonte própria de energia, a etiqueta passiva utiliza o campo eletromagnético emitido pelo sinal do leitor para se energizar. A comunicação sempre é iniciada pelo leitor, mesmo que a etiqueta ainda possua energia suficiente armazenada de uma comunicação anterior. As etiquetas semi-passivas possuem características presentes nas etiquetas passivas, como a impossibilidade de iniciar uma comunicação e características das etiquetas ativas como possuir fonte própria de energia. As etiquetas ativas são aquelas com fonte própria de energia possuindo também a característica de autonomia na comunicação, ou seja, elas podem iniciar por conta própria uma comunicação, podendo também possuir sensores integrados. Este tipo de etiqueta é geralmente utilizada em objetos de alto valor agregado (Finkenzeller et al., 2010).

Com relação à classificação quando à frequência, observa-se na Tabela 2.2 que quanto menor a frequência de operação menor o alcance. O que de fato definirá a escolha da frequência de operação é o contexto da aplicação. Por exemplo, etiquetas de Baixa Frequência são muito utilizadas em controle de acesso e identificação animal, por exemplo em um cenário onde funcionários de uma empresa aproximam a etiqueta do leitor, localizado na fechadura da porta. Etiquetas de Alta Frequência já adaptam-se melhor em cartões inteligentes. Como exemplo tem-se o controle de entrada e saída de alunos de uma sala de aula, onde cada aluno carrega um cartão no bolso e o leitor localizado na lateral da porta de entrada identifica todas as entradas e saídas. Já as de Ultra Alta Frequência (UHF) são melhor aplicadas a contextos de logística, rastreamento e localização. São as mais utilizadas comercialmente devido à aplicabilidade diversa além de usarem padronização bem definida (Finkenzeller et al.

\footnotetext{
${ }^{3}$ Informações extraídas de uma busca no sítio eletrônico http://www.aliexpress.com em Agosto de 2015.
} 
Tabela 2.2: Classificação quanto à faixa de frequência de operação (Hanada e Kudou, 2013)

\begin{tabular}{|c|c|}
\hline Tipo de etiqueta & Características \\
\hline Baixa Frequência & $\begin{array}{l}\text { Operam na faixa de frequências de } 125-134 \mathrm{kHz} \text {, al- } \\
\text { cançando em média } 50 \mathrm{~cm} \text { com taxa de transmissão de } \\
\text { até } 1 \mathrm{kbit} / \mathrm{s}\end{array}$ \\
\hline Alta Frequência & $\begin{array}{l}\text { Operam na faixa de frequências de } 13.56 \mathrm{MHz} \text {, alcan- } \\
\text { çando em média } 150 \mathrm{~cm} \text { com taxa de transmissão de até } \\
25 \mathrm{kbit} / \mathrm{s}\end{array}$ \\
\hline Ultra Alta Frequência & $\begin{array}{l}\text { Operam na faixa de frequências de } 860-960 \mathrm{MHz} \text {, al- } \\
\text { cançando em média de } 3 \mathrm{~m} \text { a } 20 \mathrm{~m} \text { com taxa de transmis- } \\
\text { são de até } 30 \mathrm{kbit} / \mathrm{s}\end{array}$ \\
\hline Micro-ondas & $\begin{array}{l}\text { Operam na faixa de frequências de } 2,45 G H z \text {, alcan- } \\
\text { çando em média } 10 \mathrm{~m} \text { com taxa de transmissão de até } \\
100 \mathrm{kbit} / \mathrm{s}\end{array}$ \\
\hline
\end{tabular}

, 2010) e alcance muito superior às demais citadas. Uma aplicação prática destes tipos de etiquetas está na abertura automática da cancela em postos de pedágios, onde as etiquetas são afixadas nos para-brisas dos automóveis e os leitores estão posicionados ao lado das cancelas. Finalmente, as etiquetas com maior taxa de transmissão e alcance são as que operam na frequência de micro-ondas, sendo estas também as de maior custo (Klair et al., 2010).

Os leitores são os responsáveis por solicitar informações das etiquetas (código de identificação). Um leitor envia energia através de ondas de rádio para as etiquetas e aguarda as respostas. As etiquetas detectam os comandos de requisição e respondem com a informação requerida, na maioria dos casos, seu código único de identificação, também chamado de código EPC (Eletronic Product Code - Código Eletrônico de Produto). Em sistemas simples, a energia enviada pelos leitores serve de gatilho "liga-desliga" para as etiquetas. No entanto, em sistemas mais complexos os leitores podem enviar até mesmo comandos de escrita para a memória das etiquetas (Finkenzeller et al., 2010). A Figura $2.4^{4}$ ilustra três diferentes tipos de leitores. Em (1) tem-se um leitor que opera na frequência UHF, com interface USB e alcance de pouco mais de $80 \mathrm{~cm}$. Em (2) ilustra-se um leitor UHF móvel, com alimentação de energia na forma de bateria e interface serial. O mesmo assemelha-se no formato a uma máquina leitora de cartões de créditos. Em (3) tem-se um exemplo de leitor UHF fixo,

\footnotetext{
${ }^{4}$ Fotos extraídas do sítio eletrônico http://www.aliexpress.com. Agosto de 2015
} 
geralmente instalado em paredes ou suportes especiais. O mesmo pode possuir alcance de 3 a 20 metros, dependendo do modelo, com interface também serial. Leitores que operam nas frequências 13,56 MHz e $125 \mathrm{KHz}$ geralmente possuem formato parecido ao mostrado na Figura 2.3 (1), podendo também aparecerem acoplados a outros dispositivos como uma fechadura ou entrada de uma porta, por exemplo (Finkenzeller et al., 2010).

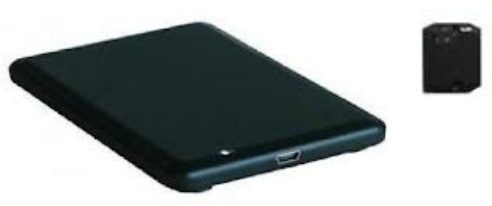

(1)

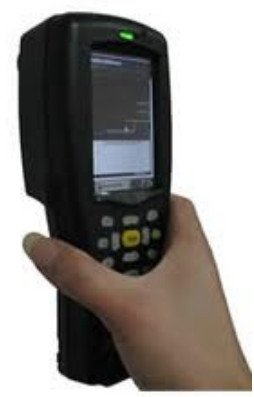

(2)

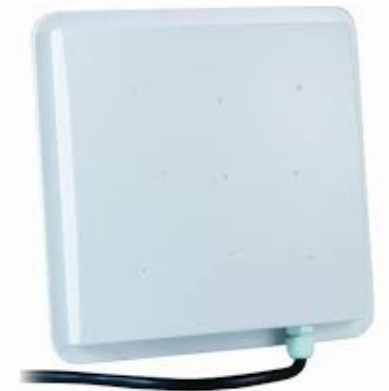

(3)

Figura 2.4: Leitores RFID em diferentes formatos.

Ao contrário das etiquetas, os leitores podem realizar tarefas de computação mais complexas, uma vez que podem estar acoplados fisicamente a sistemas computacionais robustos. Leitores com essa conexão física são chamados de leitores fixos. Quando não há a conexão com computadores os leitores são chamados de leitores móveis, que podem possuir limitações quanto à energia, o que limita tarefas computacionais a serem realizadas. Esta limitação pode ser minimizada por meio da interligação do leitor com um sistema computacional através de uma rede local, permitindo desta forma que tarefas complexas sejam realizadas fora do hardware do leitor (Dobkin, 2012).

Adicionalmente à frequência de operação escolhida, etiquetas e leitores precisam seguir um padrão de comunicação específico para tornar possível a comunicação entre ambos. Este padrão define a linguagem utilizada para a troca de mensagens. Os protocolos mais comuns e utilizados mundialmente são apresentados na Tabela 2.3 (Klair et al., 2010).

Leitores e etiquetas nunca são utilizados independentemente. Ambos fazem parte de um sistema, com características dependentes da aplicação. Os objetivos do Sistema Computacional é transformar os dados coletados das etiquetas em informações relevantes para o contexto da aplicação em uso. Por exemplo, em um contexto de Internet das Coisas, os identificadores coletados dos objetos podem ser transformados, pelo sistema computacional, 
Tabela 2.3: Protocolos para as diferentes frequências de operação de RFID

\begin{tabular}{cl}
\hline Tipo de etiqueta & Protocolo \\
\hline Baixa Frequência & ISO 18000-2 \\
Alta Frequência & ISO 18000-3 \\
Ultra Alta Frequência & ISO 18000-6 (Classe 1 Geração 2 - C1G2) \\
Micro-ondas & ISO 18000-4 \\
\hline
\end{tabular}

em informações como localização dos objetos dentro de uma organização, através de uma aplicação disponível na Internet. Dessa forma, o usuário final lida apenas com informações do tipo: Objeto A está na Sala $Y$, ao invés de lidar com o identificador numérico. Assim o Sistema Computacional exerce papel importante dentro do sistema RFID, uma vez que o mesmo faz o processamento dos dados coletados transformando-os em informações de alto nível para os usuários do sistema (Welbourne et al., 2009).

\subsection{Protocolos de Acesso ao meio}

Em qualquer rede de difusão, uma questão fundamental é determinar quem tem o direito de usar o canal quando há uma disputa por ele. Quando apenas um único canal está disponível, a determinação de quem vai ser o próximo a transmitir deve ser cuidadosamente definida. Os protocolos usados para determinar quem será o próximo em um canal de multiacesso pertencem a uma subcamada da camada de enlace de dados, conhecida como MAC (Medium Access Control - Controle de Acesso ao Meio) (Tanenbaum, 2010).

Em redes de computadores domésticas e corporativas, onde as estações estão equipadas com placas de rede Ethernet e/ou Wifi, o acesso ao meio é realizado por protocolos chamados de CSMA (Carrier Sense Multiple Access - Acesso Múltiplo com Detecção de Portadora). Em resumo, o protocolo CSMA define que as estações devem escutar o meio antes de iniciar a transmissão. Em algum momento, caso ocorra uma colisão, as estações terão a capacidade de detectá-la. Caso o canal esteja ocupado na tentativa de transmissão, a estação espera um tempo aleatório antes de uma nova tentativa (Tanenbaum, 2010). Os nós em questão precisam possuir capacidade computacional para realizar os passos descritos anteriormente. 
Por outro lado, dispositivos com pouco poder computacional e poucos recursos de hardware, como por exemplo as etiquetas RFID passivas, dispõem de limitados recursos, inviabilizando mecanismos como o CSMA de serem utilizados. Dessa forma, tem-se que etiquetas RFID de uso em massa (passivas) utilizam o método mais simples para acessar o meio: transmitir assim que houver necessidade (Finkenzeller et al., 2010).

\subsection{Resolução de Colisões em Sistemas RFID Passivos}

Quando há a presença de várias etiquetas sob o alcance de um leitor, conforme ilustrado na Figura 2.5, as mesmas comumente responderão as requisições simultaneamente, havendo a possibilidade de ocorrerem colisões (Wu et al., 2013). Como resultado das colisões que surgem durante a identificação das etiquetas há o desperdício de energia, largura de banda e tempo. Assim, para minimizar estes problemas, os leitores RFID devem implementar algum mecanismo anticolisão (Klair et al., 2010). Cenários futurísticos como a Internet das Coisas exigirão cada vez mais um melhor desempenho de todas as tecnologias envolvidas, especialmente o tratamento de colisões em sistemas RFID, pois, segundo Klair et al. (2010), esses mecanismos definem o desempenho global da tecnologia.

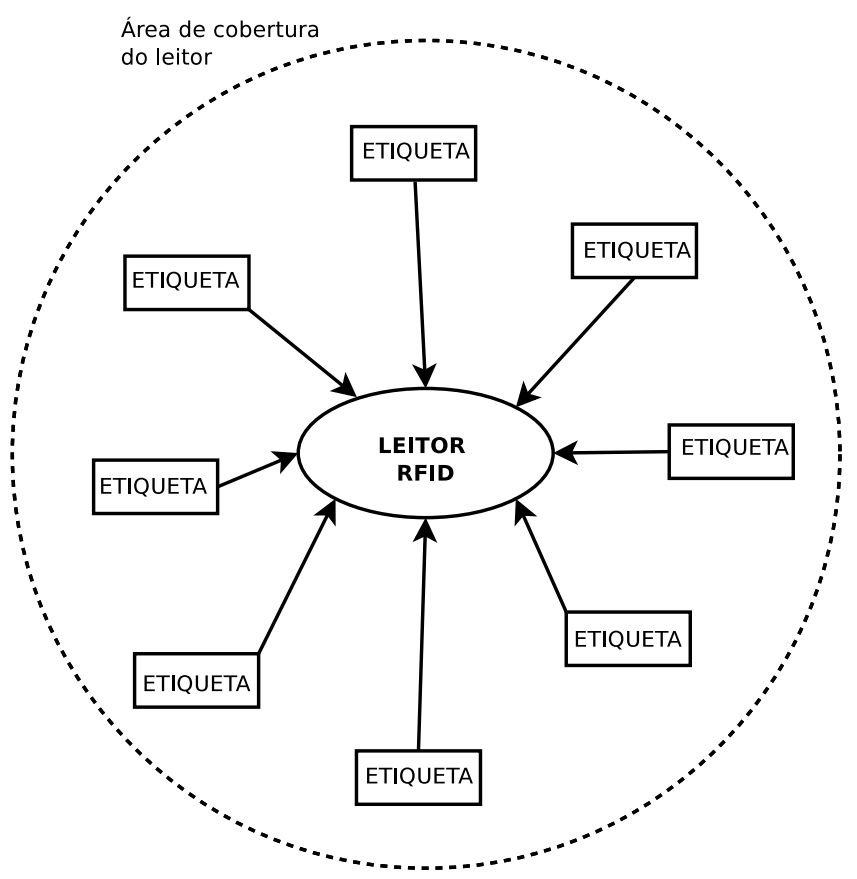

Figura 2.5: O problema das colisões em sistemas RFID.

Diferentemente de redes de computadores tradicionais como aquelas que seguem os pa- 
drões 802.3 e 802.11, os sistemas RFID possuem energia e poder computacional limitados (Finkenzeller et al., 2010), sendo inviável a implementação de mecanismos anticolisão como o CSMA/CD, que detecta colisões ou CSMA/CA, que emprega mecanismos para evitar as colisões. Dessa forma, em geral são utilizados mecanismos que seguem o padrão de divisão por tempo para o acesso ao meio, conhecidos como TDMA (Time Division Multiple Access - Acesso Múltiplo por Divisão de Tempo). Os mecanismos TDMA para RFID são divididos em dois grandes conjuntos principais: controlados pelas etiquetas ou controlados pelos leitores. A literatura especializada trata na maioria dos casos o segundo grande conjunto, sendo rara a utilização de mecanismos guiados pelas etiquetas, devido ao poder computacional limitado das mesmas (Klair et al., 2010). A Figura 2.6 apresenta a classificação dos principais algoritmos anticolisão guiados pelo leitor para sistemas RFID. As caixas com fundo cinza representam o escopo dos algoritmos de acesso ao meio desta tese.

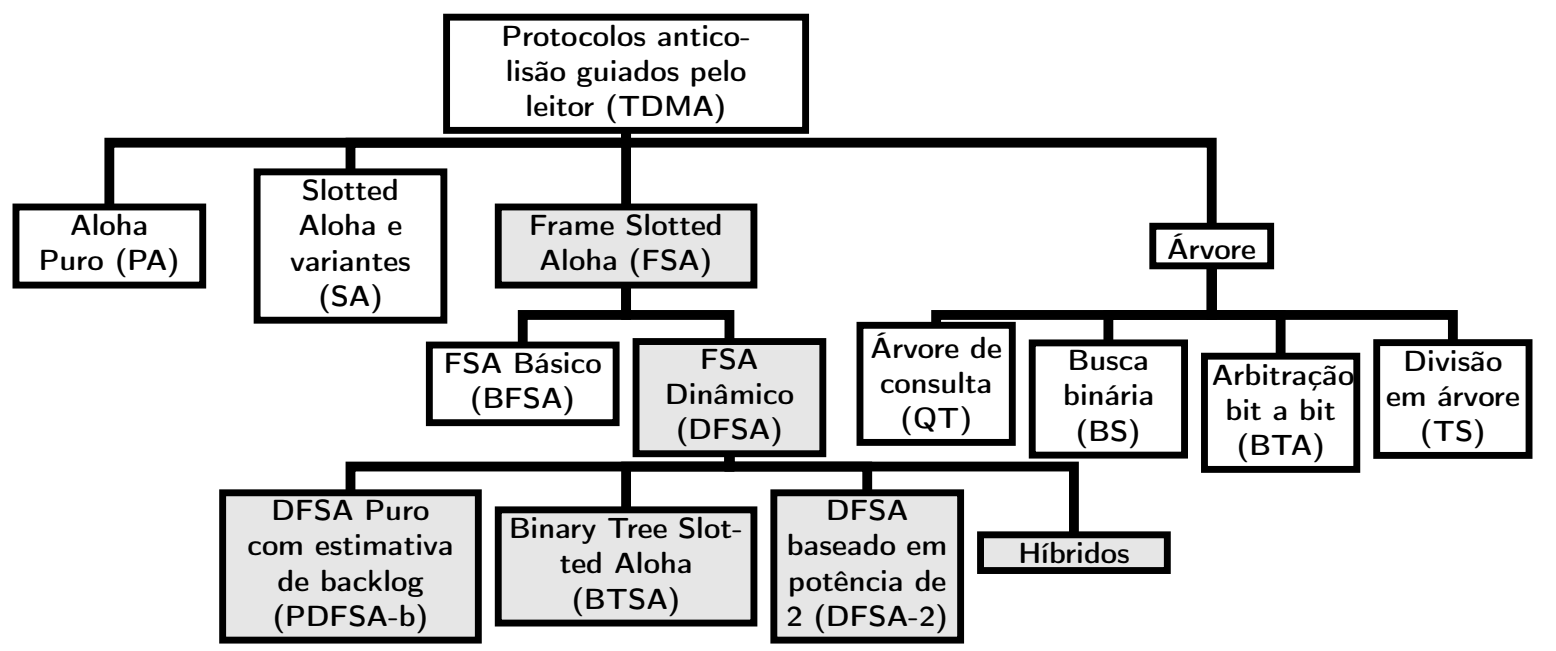

Figura 2.6: Taxonomia de protocolos/algoritmos anticolisão para sistemas RFID.

Em sistemas RFID Pure Aloha (PA) uma etiqueta responde com seu identificador (ID) após ser energizada pelo leitor. A mesma espera pela resposta do leitor que pode ser uma confirmação positiva (ACK), indicando que o ID foi recebido corretamente ou uma confirmação negativa (NACK), sinalizando uma colisão. Se duas ou mais etiquetas transmitem simultaneamente, uma colisão total ou parcial ocorre, sendo resolvida pelo leitor através da solicitação de que as etiquetas em questão retransmitam seus IDs após esperarem um tempo aleatório (Finkenzeller et al., 2010) (Klair et al., 2010).

Os mecanismos baseados em Slotted Aloha operam com as etiquetas enviando seus iden- 
tificadores em slots de tempo síncronos. Caso haja colisão em determinado slot, as etiquetas em colisão aguardam um tempo aleatório e tentam novamente a transmissão. O processo termina quando todas as etiquetas são identificadas (Schwartz, 1987), ou seja, quando não houver slots de colisão. Por outro lado, os algoritmos baseados em Frame Slotted Aloha (FSA) dividem o tempo em quadros com $n$ slots de tempo, no qual cada etiqueta escolhe um slot aleatoriamente. O leitor solicita informando o slot atual, um de cada vez, e as etiquetas respondem no momento em que o slot escolhido for chamado pelo leitor. Caso duas ou mais etiquetas escolham um mesmo slot, ocorre uma colisão. Quando todos os slots forem chamados, o processo é reiniciado com um novo quadro até que todas as etiquetas sejam identificadas. O que diferencia o Basic Frame Slotted Aloha (BFSA) do Dynamic Frame Slotted Aloha (DFSA) é respectivamente a manutenção ou não do tamanho do próximo quadro (Zhu e Yum, 2011) (Ullah et al., 2012) (Shih et al., 2006) (Klair et al., 2010).

Os algoritmos baseados em busca em árvores (Tree) (Capetanakis, 1979), também chamados de Splitting, funcionam através da divisão do conjunto de etiquetas em $x$ subgrupos, sendo $x>1$. O leitor inicialmente envia um pacote a todas as etiquetas em sua área de cobertura, gerando colisões. Neste momento as etiquetas selecionam um número aleatório entre 0 e $B-1$. Suponha que $B=2$, então as etiquetas selecionam 0 ou 1 . Assim, divide-se o conjunto de etiquetas em dois grupos, as que selecionaram 0 e as que selecionaram 1 . O leitor continua dividindo cada um dos ramos da árvore até que apenas uma etiqueta responda, quando o mesmo segue voltando até a raiz da árvore. Quando toda a árvore gerada for identificada, o processo termina. As diversas propostas diferem em qual estratégia utilizar para gerar a árvore e para dividir os nós em colisão. Essa categoria é computacionalmente mais complexa de se implementar e geralmente leva a um maior tempo de identificação quando comparada com os mecanismos baseados em ALOHA (Klair et al., 2010).

A Figura 2.7 mostra um exemplo detalhado do funcionamento básico do (D)FSA. Em I é mostrado como se faz o processo de identificação do conjunto de etiquetas sob o alcance do leitor. Este processo é dividido em $i$ quadros de tamanhos $\left(L_{i}\right)$, variáveis no caso do DFSA, e de tamanhos iguais no caso do BFSA . Cada quadro possui um tamanho $L_{i}$, que define a quantidade de slots de tempo. Um slot é definido como um intervalo de tempo que uma etiqueta escolhe para responder em determinado momento ao leitor. O progresso do processo 
de identificação é igual para cada um dos quadros: antes do leitor chamar o primeiro slot do quadro, cada uma das etiquetas escolhe aleatoriamente um slot no intervalo $[1, L]$ (ou $[0, L-1])$. Posteriormente o leitor envia requisições de identificação em frequências de cada um dos slots. Caso nenhuma etiqueta responda em um determinado slot $z$, ele é chamado de slot vazio, conforme mostrado em II. Já se mais de uma etiqueta responder dentro de um mesmo slot ele é chamado slot de colisão (em III). Na situação onde apenas uma etiqueta responde, ele é chamado de slot de sucesso, sendo a etiqueta que respondeu devidamente identificada (em IV) (He e Wang, 2013).
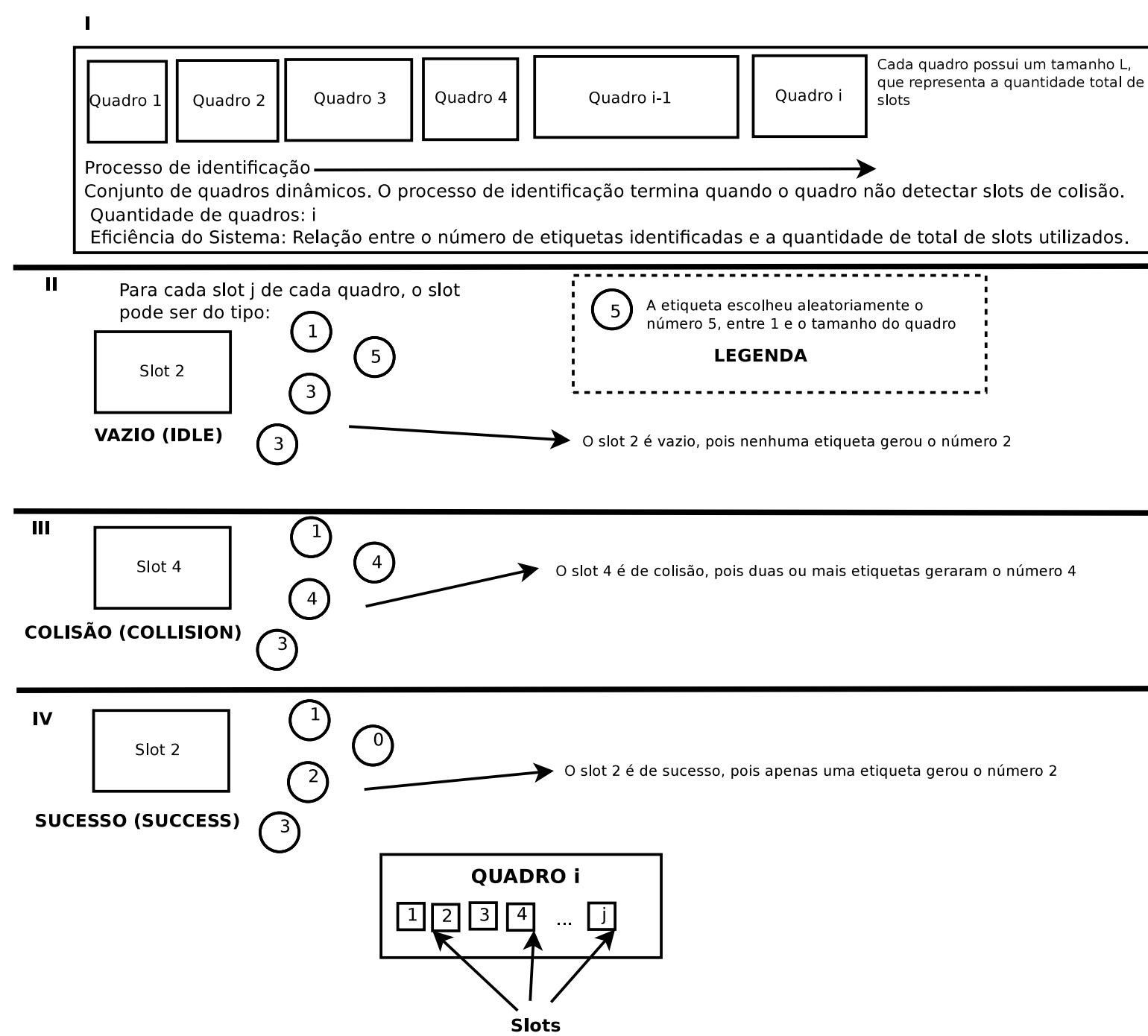

Figura 2.7: Exemplo de funcionamento do (D)FSA.

Os algoritmos Frame Slotted Aloha - FSA, destacados na Figura 2.6 seguem o funcionamento básico descrito pelo Algoritmo 1. Inicialmente define-se o tamanho do quadro chamado de $L$ (linha 2 do Algoritmo 1). As etiquetas escolhem aleatoriamente um slot para 
transmitir entre $[1, L]$. Posteriormente o leitor envia requisições a cada slot de tempo (linhas 7 e 8). Se ao final do quadro forem identificados slots de colisão (linhas 12 a 14 e 18 a 21), um novo quadro de tamanho $L$ é criado (linhas 18 a 21), as etiquetas em colisão escolhem um novo slot entre $[1, L]$ e o leitor reinicia o procedimento, até que nenhum slot de colisão seja identificado (laço das linhas 3 a 22). O desempenho do mecanismo FSA está diretamente relacionado com a proximidade do tamanho do quadro com a quantidade de etiquetas a serem identificadas. Como em geral a quantidade de etiquetas é desconhecida, a estimativa do tamanho inicial do quadro é um fator chave no desempenho global do mecanismo. Os mecanismos dinâmicos (DFSA) fazem um melhoramento em relação ao BFSA atacando o problema do tamanho dos quadros subsequentes. Os mecanismos Dynamic Frame Slotted Aloha - DFSA, a partir da quantidade de slots de colisão, fazem um cálculo estimando o número restante de etiquetas (linhas 19 e 20), para otimizar o quadro seguinte. Este cálculo é realizado após cada final de quadro (laço das linhas de 7 a 15) até que nenhum slot de colisão seja identificado (linhas 16 a 18). Assim, o cálculo do tamanho do quadro seguinte (backlog) possui papel chave no desempenho do processo de identificação já que quadros muito grandes em relação ao número de etiquetas gerarão muitos slots vazios e quadros muito pequenos em relação ao número de etiquetas gerarão muitas colisões.

\subsection{Probabilidade e Distribuição Binomial}

O desenvolvimento de protocolos anticolisão baseados em ALOHA fundamenta-se nos princípios e definições da teoria da probabilidade, que é o ramo da matemática concentrado nos fenômenos aleatórios, processos estocásticos e eventos não determinísticos. O correto estudo e entendimento destes conceitos leva várias propostas sobre como lidar com o problema das colisões em sistemas RFID, conforme mostra o Capítulo 3.

A probabilidade de que um evento ocorra exatamente $x$ vezes em $n$ tentativas independentes, com probabilidade de sucesso definida por $p$ e probabilidade de falha definida por $(q=(1-p))$ é dada pela Equação 2.1 (Spiegel et al., 2002).

$$
f(x)=P(X=x)=\left(\begin{array}{l}
n \\
x
\end{array}\right) \times p^{x} \times q^{n-x}
$$




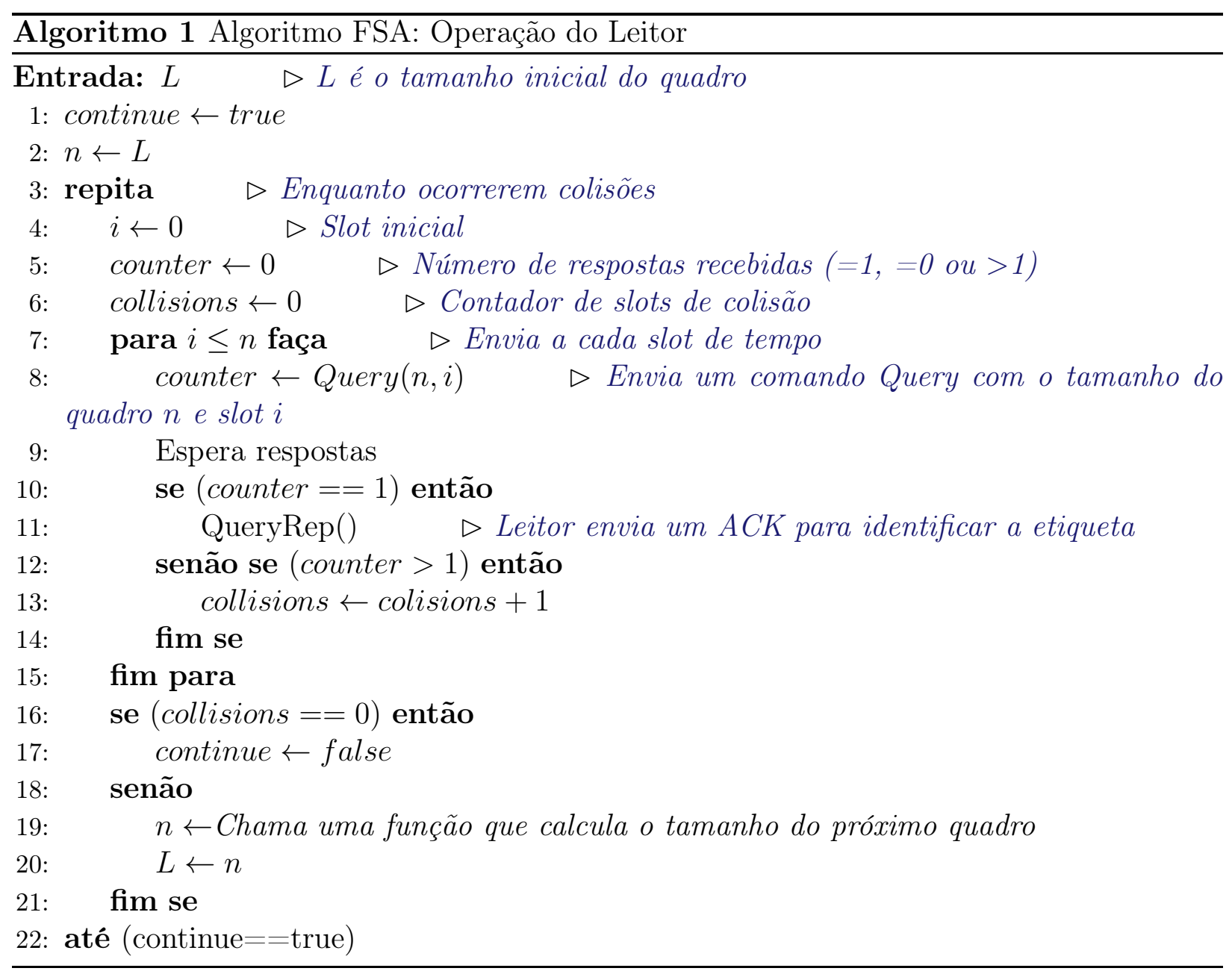

Seja $L$ o tamanho do quadro e $n$ a quantidade de etiquetas, então, tem-se que a ocupação do quadro por parte das etiquetas segue uma distribuição binomial com $n$ tentativas e probabilidade de sucesso de $\frac{1}{L}$ (Wu et al., 2013). A partir da definição de distribuição binomial (Equação 2.1), a Equação 2.2 define a probabilidade de que dentre as $n$ etiquetas, $Y$ delas selecionem o mesmo slot de um quadro de tamanho $L$.

$$
p(X=Y)=\left(\begin{array}{c}
n \\
Y
\end{array}\right) \times\left(\frac{1}{L}\right)^{Y} \times\left(1-\frac{1}{L}\right)^{n-Y}
$$

Wu et al. (2013) reescreve a Equação 2.2 com a notação expressa pela Equação 2.3.

$$
p(L, n, Y)=\left(\begin{array}{c}
n \\
Y
\end{array}\right) \times\left(\frac{1}{L}\right)^{Y} \times\left(1-\frac{1}{L}\right)^{n-Y}
$$

Lê-se a Equação 2.3 como: A probabilidade de que dentre as $n$ etiquetas, $Y$ selecionem o mesmo slot em um quadro de tamanho $L$. Dessa forma temos que as probabilidades de que um determinado slot seja de sucesso, vazio e de colisão seguem os Lemas 1, 2 e 3. 
Lema 1. Probabilidade de que determinado slot seja vazio (idle):

$$
p(X=0)=\left(1-\frac{1}{L}\right)^{n}
$$

A partir de $(2.2)$

Demonstração. A partir dos conceitos de distribuição binomial (Equação 2.2) temos que: $p(X=0)=\left(\begin{array}{l}n \\ 0\end{array}\right) \times\left(\frac{1}{L}\right)^{0} \times\left(1-\frac{1}{L}\right)^{n-0} \rightarrow p(X=0)=\left(1-\frac{1}{L}\right)^{n}$

Lema 2. Probabilidade de que determinado slot seja ocupado por uma única etiqueta (success):

$$
p(X=1)=\frac{n}{L} \times\left(1-\frac{1}{L}\right)^{n-1}
$$

A partir de (2.2)

Demonstração. $p(X=1)=\left(\begin{array}{c}n \\ 1\end{array}\right) \times \frac{1}{L}^{1} \times\left(1-\frac{1}{L}\right)^{n-1}$

$p(X=1)=n \times \frac{1}{L} \times\left(1-\frac{1}{L}\right)^{n-1} \Rightarrow \frac{n}{L} \times\left(1-\frac{1}{L}\right)^{n-1}$

Lema 3. Uma colisão ocorre quando 2 ou mais etiquetas selecionam o mesmo slot, ou seja, a probabilidade de que um slot seja de colisão é definida como:

$$
p(X>1)=(1-p(X=0)-p(X=1))
$$

A partir de $(2.2)$

Demonstração. Como existem apenas três tipos diferentes de slots, sucesso $(p(X=1))$, colisão $(p(X>1))$ e vazio $(p(X=0))$, podemos chegar à definição da probabilidade de um slot de colisão como a probabilidade de que não ocorram os outros dois tipos de slots.

Definição 1. Os valores esperados da quantidade de slots vazios, sucesso e colisão são definidos pelas Equações (2.4) (2.5),(2.6) e (2.7).

$$
\begin{gathered}
E(L, n, k)=L \times\left(\left(\begin{array}{l}
n \\
k
\end{array}\right) \times\left(\frac{1}{L}\right)^{k} \times\left(1-\frac{1}{L}\right)^{n-k}\right) \\
E(L, n, 0)=L \times\left(1-\frac{1}{L}\right)^{n} \\
E(L, n, 1)=L \times\left(n \times\left(\frac{1}{L}\right) \times\left(1-\frac{1}{L}\right)^{n-1}\right)
\end{gathered}
$$




$$
E(L, n, k)=L \times(1-p(L, n, 0)-p(L, n, 1))
$$

O exemplo A.0.6 do Apêndice A ilustra uma aplicação dos conceitos.

\subsection{Simuladores RFID}

A simulação de redes é uma ferramenta essencial no desenvolvimento e na avaliação de desempenho de redes de comunicação de dados, especialmente no emprego de novos protocolos e tecnologias (Bouras et al., 2013). Os chamados simuladores de redes permitem que uma rede de computadores arbitrária seja modelada através da especificação do comportamento dos nós e dos canais de comunicação. Por exemplo, quando deseja-se investigar um novo algoritmo/protocolo anticolisão para sistemas RFID, normalmente implementa-se o mesmo em um simulador. Posteriormente, o comportamento do protocolo pode ser estudado sob diferentes tipos de topologias e/ou cenários através da modificação de alguns parâmetros de simulação (Weingartner et al., 2009).

Em se tratando de simulação de sistemas RFID, algumas soluções existentes, como o RfidSim e Rifidi cumprem bem alguns objetivos específicos para avaliação de funcionamento de redes com leitores e etiquetas RFID (Weingartner et al., 2009) (Fritz et al. , 2012). A principal característica dos simuladores Rifidi (Zhang et al., 2012a) e RfidSim (Floerkemeier e Sarma, 2009) é a possibilidade de estudar o comportamento de um sistema RFID arbitrário com um número qualquer de etiquetas, seguindo o protocolo ISO/IEC 18000-6C (EPCglobal, GS1 Inc., 2015). O simulador Network Simulator 2 ou ns-2 não é específico para uma tecnologia em particular, podendo ser utilizado para o estudo de diversas tecnologias diferentes e até mesmo a integração entre as mesmas (Lessmann et al., 2008).

O protocolo de comunicação ISO/IEC 18000-6C foi implementado no simulador RfidSim (Floerkemeier e Sarma, 2009) para fins de avaliação e testes de sistemas RFID. A aplicação baseia-se no paradigma de eventos discretos. O principal objetivo do simulador é a avaliação da camada física e da camada de aplicação, permitindo a utilização de até 10.000 (dez mil) etiquetas. O autor valida a proposta através da comparação de parâmetros da camada física em simulações com valores reais. Apesar de apresentar-se como uma solução completa e 
eficiente, a mesma não é adequada para estudos e avaliação da camada de acesso ao meio, uma vez que o mesmo é adequado para simulação da camada de aplicação, sendo portanto não utilizada na literatura sobre mecanismos anticolisão.

O simulador Rifidi (Huebner et al., 2012) permite a virtualização de leitores de vários fabricantes existentes para o teste e avaliação de aplicações RFID. Está disponível publicamente desde 2006 no sítio da SourceForge ${ }^{5}$. A aplicação consiste de três ferramentas principais denominadas de emulador, designer e tag streamer. O emulador consiste de uma ferramenta gráfica que permite o controle e interação de leitores e etiquetas. O designer consiste de uma ferramenta para modelagem de cenários em 3D que não é mais suportada atualmente. A modelagem da aplicação é de responsabilidade também do emulador. O tag streamer é uma ferramenta de análise de desempenho que permite que seja feito um estudo do comportamento de uma aplicação RFID modelada. Apesar de cumprir bem o papel de simular aplicações RFID reais e modelar dispositivos físicos reais, o mesmo tem como objetivo principal a avaliação da camada de aplicação. Sua utilização com fins de avaliação e estudo do funcionamento das camadas inferiores como a camada de acesso ao meio (anticolisão) não é adequada.

O simulador ns-2, baseado em eventos discretos, é uma das aplicações mais utilizadas pela literatura especializada em avaliação de desempenho de redes de computadores. Desde sua publicação em 1996, tornou-se praticamente o padrão da simulação de redes. Este fato pode ser atribuído ao fato de que vários modelos como protocolos e geradores de tráfegos estão publicamente disponíveis no ns-2 (Weingartner et al., 2009). Apesar de possuir alguns problemas relacionados a escalabilidade, continua até hoje (2015), como sendo o simulador mais utilizado pelo meio acadêmico em diversas subáreas de redes de computadores sem fio como WiMax, MANET, Redes de Sensores Sem Fio - RSSF, Comunicações Via Satélite, entre várias outra áreas (Ou e He, 2013) (Verma et al., 2013) (Song et al., 2013) (Zhou et al. , 2013) (Kang et al., 2012) (Zhang et al., 2012b) (Freitag e da Fonseca, 2007) (Wang et al., 2010).

\footnotetext{
${ }^{5}$ http://sourceforge.net/projects/rifidi/ - Acessado em Agosto de 2015
} 


\subsection{Efeito Captura}

O efeito captura refere-se à correta identificação de uma etiqueta mesmo que ela esteja em um slot de colisão (Li e Wang, 2011) (Wu e Zeng, 2015). A Figura 2.8 ilustra esta situação. Os primeiros três slots são corretamente identificados como sucesso, colisão e vazio respectivamente . No slot de número quatro, três etiquetas respondem à requisição do leitor e o leitor identificou uma etiqueta como sucesso, ao invés de detectar uma colisão. Dessa forma, mecanismos tradicionais de estimativa de tamanho de quadro (protocolos PDFSAb) falham em funcionar corretamente, resultando em cálculos comprometidos (Li e Wang , 2011) (Wu e Zeng, 2015). Como resultado, uma nova abordagem para lidar com o problema deve ser estudada. Poucos trabalhos têm sido publicados para lidar com o fenômeno do efeito captura em protocolos FSA (Shin e Kim, 2009) (Natali et al., 2010) (Li e Wang, 2011) (Choi et al., 2012) (Wu e Zeng, 2015) . Portanto, o problema do efeito captura ainda requer muito estudo e pesquisa aprofundada, uma vez que o problema é muito comum na prática (Prodanoff, 2010).

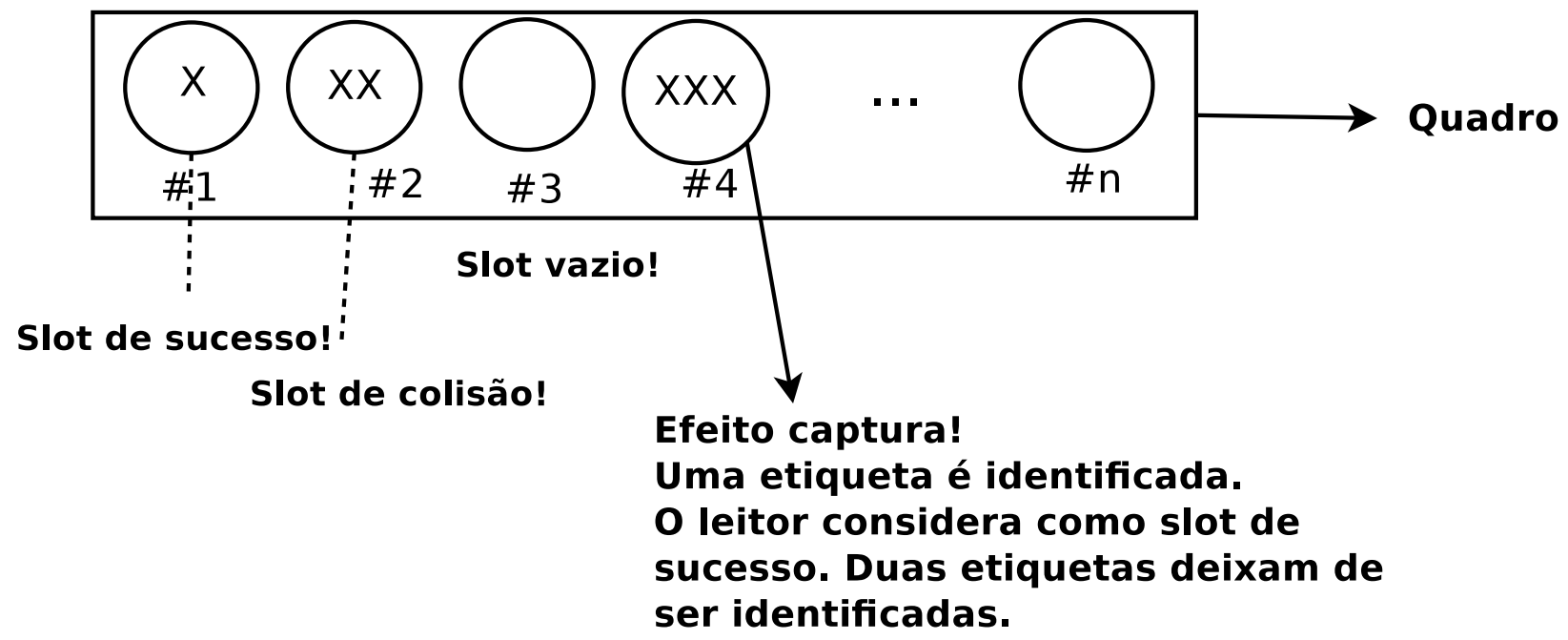

Figura 2.8: Exemplo de efeito captura

\subsection{Notações e acrônimos}

A Tabela 2.4 descreve os acrônimos e notações utilizadas no restante desta tese com o objetivo de identificar os parâmetros necessários para o detalhamento de cada uma das métricas de desempenho que serão apresentadas ao longo dos próximos capítulos. 
Tabela 2.4: Descrição de acrônimos.

\begin{tabular}{|c|c|}
\hline Notação & Descrição \\
\hline $\bar{n}$ & Número de etiquetas \\
\hline$S_{e f}$ & Eficiência do sistema $([0,1])$ \\
\hline$S_{e f}^{q}$ & Eficiência do sistema do algoritmo $Q([0,1])$ \\
\hline$S_{e f}^{X}$ & Eficiência do sistema do protocolo $\mathrm{X}$ (X pode ser qualquer protocolo) $(0-1)$ \\
\hline thr & Vazão (\%), ou eficiência do sistema na escala de $0-100$. \\
\hline$V$ & Tensão do leitor (Volts) \\
\hline$I$ & Corrente do leitor (Amperes) \\
\hline Total $_{\text {slots }}$ & $\begin{array}{l}\text { Quantidade total de slots utilizados durante a identificação do conjunto de etiquetas (times- } \\
\text { lots) }\end{array}$ \\
\hline suc ou $S$ ou $S_{1}$ & Número de slots de sucesso durante a identificação do conjunto de etiquetas (timeslots) \\
\hline col ou $C$ ou $S_{k}$ & Número de slots de colisão durante a identificação do conjunto de etiquetas (timeslots) \\
\hline$i d l$ ou $E$ ou $S_{0}$ & Número de slots vazios durante a identificação do conjunto de etiquetas (timeslots) \\
\hline$s u c_{i}$ ou $S_{i}$ & Número de slots de sucesso do quadro/ rodada $i$ (timeslots) \\
\hline $\operatorname{col}_{i}$ ou $C_{i}$ & Número de slots de colisão do quadro/ rodada $i$ (timeslots) \\
\hline$i d l_{i}$ ou $E_{i}$ & Número de slots vazios do quadro/ rodada $i$ (timeslots) \\
\hline frames & Número de quadros utilizados durante o processo de identificação do conjunto de etiquetas \\
\hline$G_{q}$ & Ganho percentual em relação ao algoritmo Q (\%) \\
\hline$B$ & Energia armazenada da bateria (Joules) \\
\hline$E_{\text {total }}$ & $\begin{array}{l}\text { Quantidade total de energia consumida durante o processo de identificação do conjunto de } \\
\text { etiquetas (Joules) }\end{array}$ \\
\hline$E_{\text {wasted }}$ & $\begin{array}{l}\text { Quantidade de energia desperdiçada devido a colisões e slots vazios durante o processo de } \\
\text { identificação do conjunto de etiquetas (Joules) }\end{array}$ \\
\hline$N_{\text {protocol }}$ & $\begin{array}{l}\text { Quantidade de vezes que um leitor móvel alimentado a bateria pode ler um conjunto de } \\
\text { etiquetas }\end{array}$ \\
\hline$T_{\text {total }}$ & $\begin{array}{l}\text { Latência. Tempo total de identificação necessário para identificar um conjunto de etiquetas } \\
\text { (segundos) }\end{array}$ \\
\hline$T_{\text {wasted }}$ & Latência desperdiçada devido a colisões e slots vazios (segundos) \\
\hline Bits total & Quantidade total de tráfego trocado entre etiquetas e leitores (bytes) \\
\hline$P_{\text {size }}^{\text {query }}$ & Tamanho do pacote Query (bits) \\
\hline$P_{\text {size }}^{\text {rnte }}$ & Tamanho do pacote $R N 16$ (bits) \\
\hline$P_{\text {size }}^{a c k}$ & Tamanho do pacote ACK (bits) \\
\hline$P_{\text {size }}^{i d}$ & Tamanho do identificador da etiqueta (bits) \\
\hline$P_{\text {size }}^{\text {queryep }}$ & Tamanho do pacote QueryRep (bits) \\
\hline $\begin{array}{l}\text { size } \\
P_{\text {size }}^{\text {quer Adjust }}\end{array}$ & Tamanho do pacote QueryAdjust (bits) \\
\hline Sppe size & Somatório dos pacotes trocados em relação aos slots de sucesso (bits) \\
\hline$S p_{\text {size }}^{\text {col }}$ & Somatório dos pacotes trocados em relação aos slots de colisão (bits) \\
\hline$S p_{s i z e}^{i d I^{c}}$ & Somatório dos pacotes trocados em relação aos slots vazios (bits) \\
\hline$S t b_{[a, b]}$ & Grau de estabilidade quando a quantidade de etiquetas varia de $a$ até $b(\%)$ \\
\hline$S_{\text {total }}^{a}$ & Quantidade total de slots utilizados durante a identificação de $a$ etiquetas (timeslots) \\
\hline & Percentual de etiquetas não identificadas devido ao efeito captura Wu e Zeng (2015) \\
\hline$n_{\text {missed }}$ & Número de etiquetas não identificadas devido ao efeito captura \\
\hline
\end{tabular}




\section{Capítulo 3}

\section{Protocolos anticolisão para sistemas}

\section{RFID passivos}

O problema das colisões em sistemas RFID pode se agravar devido ao aumento do número de etiquetas sob o alcance de um mesmo leitor, por exemplo em cenários ou aplicações envolvendo milhares de etiquetas por exemplo (Klair et al., 2010; Wu et al., 2013). As colisões resultam em desperdício de banda, energia e tempo de identificação. Para minimizar os impactos das mesmas, os leitores RFID precisam implementar mecanismos (algoritmos/protocolos) anticolisão. Dessa forma, este capítulo faz uma revisão abrangente do estado da arte sobre os protocolos anticolisão baseados em ALOHA, além de fornecer uma comparação sobre diferentes aspectos de cada uma das propostas analisadas.

O restante deste capítulo está organizado como a seguir: A Seção 3.1 descreve o mecanismo de funcionamento de cada um dos tipos de algoritmos anticolisão baseados em FSA, alvo desta tese, e suas respectivas diferenças principais. A Seção 3.2 detalha várias propostas que usam quadros de tamanho baseado em potência de 2, como por exemplo o atual padrão EPCGlobal para etiquetas classe 1 geração 2 (C1G2), seguida pelas Seções 3.3, 3.4 e 3.5 que tratam das categorias com cálculo de backlog, Binary Tree Slotted Aloha (BTSA) e híbridos. Em cada uma dessas seções inicia-se explicando os algoritmos mais citados de cada categoria e posteriormente subseções explicam os demais algoritmos. A Seção 3.6 encerra o capítulo apresentando as principais propostas que lidam com efeito captura. 


\subsection{Taxonomia}

Os protocolos FSA podem ser classificados em duas grandes categorias principais, conforme mostra a Figura 3.1. A diferença entre os dois grandes grupos é a possibilidade de alteração do tamanho dos quadros ao longo do processo de identificação. No BFSA, os quadros possuem tamanho fixo enquanto os DFSA implementam alguma estratégia para definição dos tamanhos dos quadros.

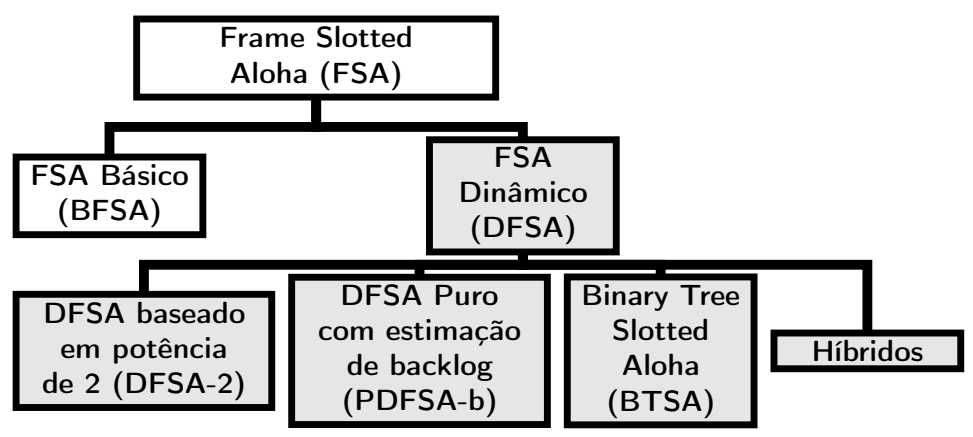

Figura 3.1: Taxonomia de protocolos/algoritmos FSA para sistemas RFID.

Na prática, apenas os classificados como DFSA são propostos e definidos na literatura, devido ao baixo desempenho oferecido pelos mecanismos BFSA. Os DFSA podem ser classificados em quatro sub-categorias principais: Os DFSA-2 dimensionam os quadros como uma potência de 2, permitindo uma forma mais rápida de aumentar ou diminuir o tamanho dos mesmos. No DFSA puro com estimação de backlog o algoritmo define ao final de cada quadro um novo tamanho para o próximo quadro. No BTSA, os slots de colisão são tratados separadamente por novos quadros de tamanho 2. Finalmente os híbridos implementam estratégias de quaisquer outras categorias.

\subsection{DFSA baseado em potência de 2 (DFSA-2)}

Protocolos de comunicação baseados em Aloha como SA (Slotted Aloha) ou FSA não são novos e foram concebidos desde as primeiras redes de computadores (Tanenbaum, 2010). Com a padronização pela EPCGlobal das etiquetas RFID mais utilizadas em diversas aplicações, foi proposto um algoritmo também baseado em FSA chamado de Algoritmo Q, ou C1G2 (EPCglobal, GS1 Inc., 2015) . Este algoritmo, por ser baseado em DFSA no qual os quadros são redimensionados dinamicamente, utiliza a seguinte estratégia para determinar 
quais etiquetas respondem em que momento, e para definir o tamanho dos quadros. Todo tamanho de quadro é definido como uma potência de dois, por padrão iniciando em 16, ou seja, tem-se o valor inicial de $Q$, o expoente da potência de 2 igual a 4 e calculamos o tamanho do quadro segundo a Equação 3.1.

A Figura 3.2 ilustra genericamente como funciona um algoritmo DFSA-2 (Arjona et al., 2015). Após as etiquetas selecionarem um slot aleatório entre $\left[0,2^{Q}-1\right]$, o leitor consulta o slot 0 . Em caso de uma única resposta, tem-se um sucesso. O leitor não modifica o valor de $Q$ e todas as demais etiquetas decrementam o valor de seus slots selecionados. Em caso de mais de uma resposta, ou seja, uma colisão, o leitor utiliza alguma estratégia para aumentar o valor de $Q$, depois envia este novo valor às etiquetas que devem selecionar outro slot aleatório. O envio de um novo valor de $Q$ também ocorre em caso de nenhuma resposta, com a diferença que neste caso o leitor decrementa o valor de $Q$. O algoritmo permanece sendo executado até que o valor de $Q$ atinja o valor zero. A ideia por trás das mudanças ou manutenções do valor de Q é que se há uma única resposta, então o tamanho do quadro está bem próximo da quantidade real de etiquetas, motivo pelo qual o valor de $\mathrm{Q}$ deve ser mantido. Se houve colisão é porque o tamanho do quadro está pequeno, por isso o valor de Q é incrementado. Caso houve um slot ocioso é porque provavelmente o tamanho do quadro está muito maior do que a quantidade de etiquetas, motivo pelo qual o valor de Q é decrementado.

\subsubsection{Algoritmo Q - C1G2}

Os Algoritmos 2 e 3 formalizam o funcionamento do Algoritmo Q (EPCglobal, GS1 Inc., 2015).

Inicialmente, define-se além do valor inteiro de $Q$, um valor de ponto flutuante também para $Q$, denominado $Q_{f p}$ (Algoritmo 2 - linha 2). Uma constante denominada de $c$ também deve ser definida no intervalo de $[0,1,0,5]$ (Algoritmo 2 - Entrada). Esta constante acelera ou atrasa o redimensionamento do tamanho do quadro. O valor padrão é definido como 0,3 , 


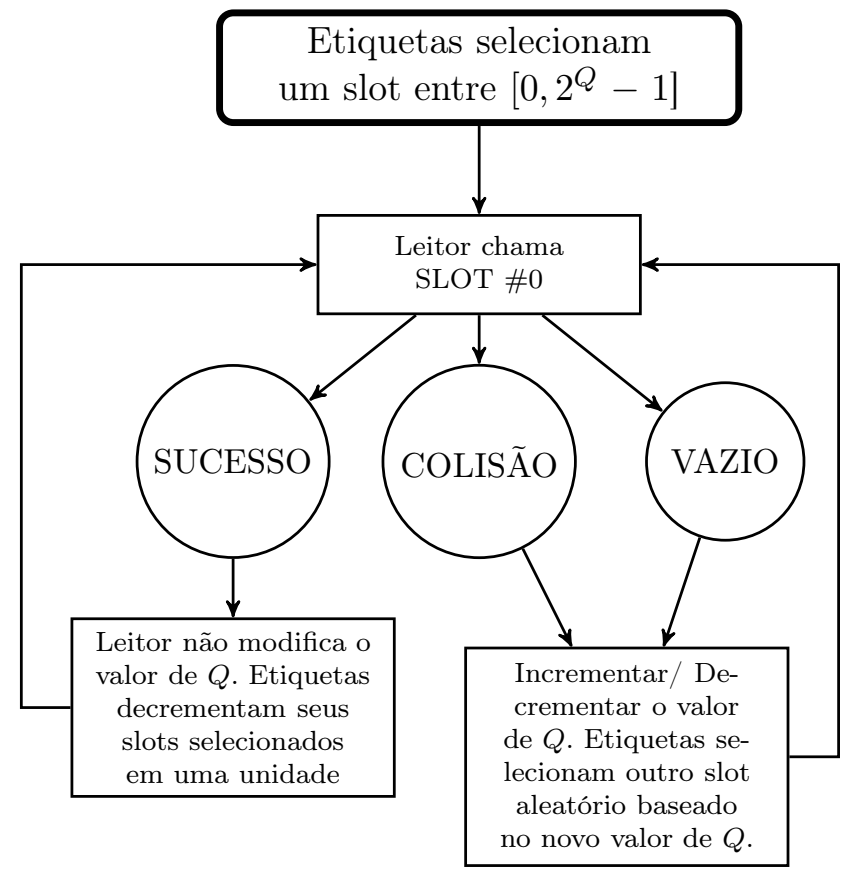

Figura 3.2: Resumo do DFSA-2.

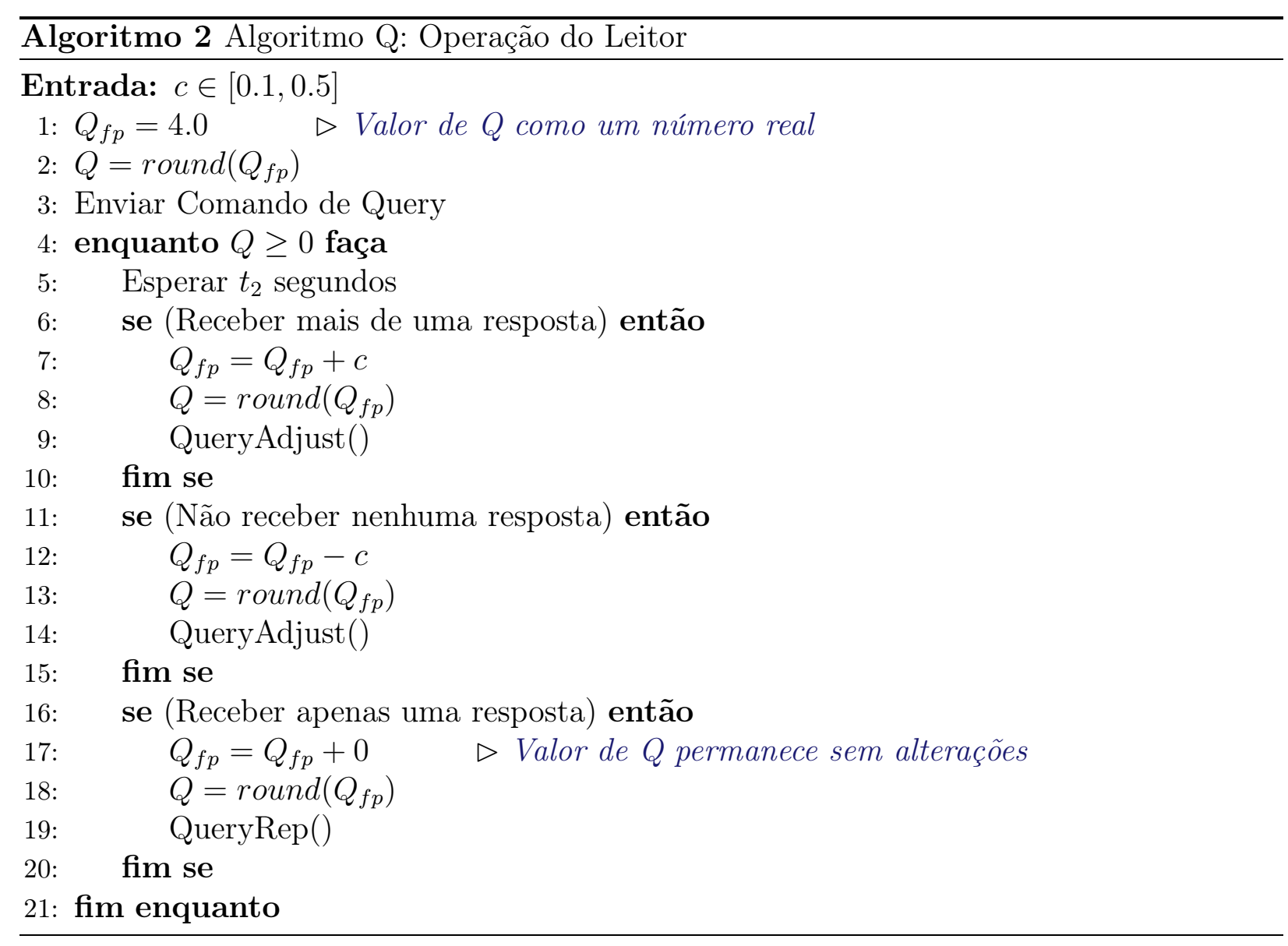

o que faz com que o algoritmo repita pelo menos uma vez o processo antes de redimensionar para um novo tamanho. Wu et al. (2013) utiliza valores de $c$ baixos quando $Q$ também é baixo, e alto quando $Q$ é alto. Antes de enviar o primeiro comando de requisição (Algoritmo 


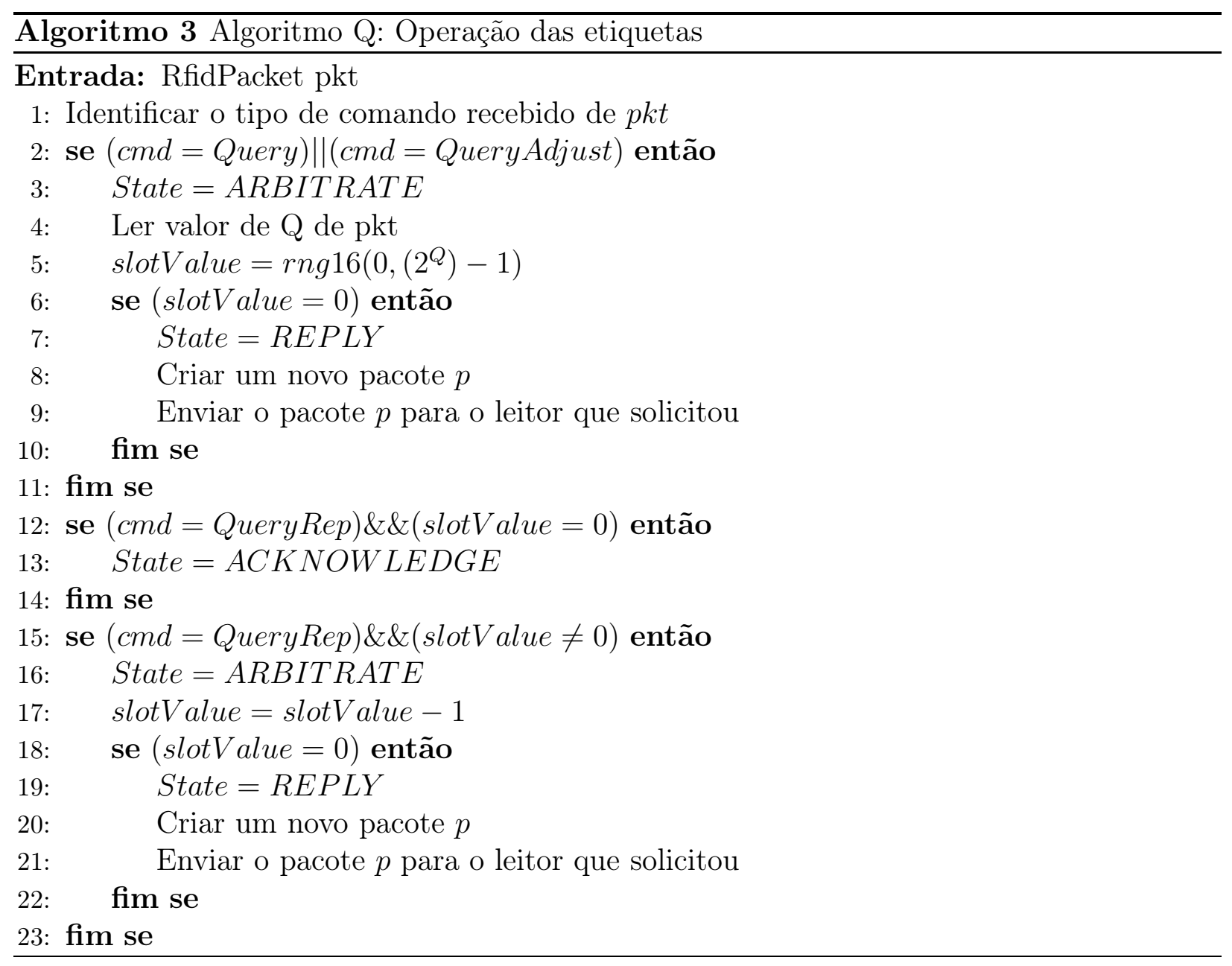

2 - linha 3), define-se $Q$ sendo igual ao inteiro mais próximo de $Q_{f p}$ (Algoritmo 2 - linha 2), que inicialmente será o mesmo valor. Posteriormente o leitor envia o comando Query (Algoritmo 2 - linha 3), que é responsável por iniciar o processo de identificação. As etiquetas ao receberem um comando Query ou QueryAdjust geram um número aleatório entre $\left[0,\left(2^{Q}\right)-1\right]$ (Algoritmo 3 - linhas 2 e 5) e mudam para o estado ARBITRATE (Algoritmo 3 - linha 3), conforme o diagrama de estados apresentado na Figura 3.3.

As etiquetas que gerarem o número 0 respondem imediatamente (Algoritmo 3 - linhas 6-10), mudando o estado para REPLY (Algoritmo 3 - linha 7 ou 19), e as demais não fazem nada. Caso o leitor receba mais de uma (Algoritmo 2 - linhas 6-10) ou nenhuma resposta (Algoritmo 2 - linhas 11-15) após $t_{2}$ segundos, o valor de $Q_{f p}$ é modificado de acordo com as Equações 3.2 e 3.3 respectivamente (Algoritmo 2 - linhas 12-13 e 17-18).

$$
Q_{f p}=\min \left(15, Q_{f p}+c\right)
$$




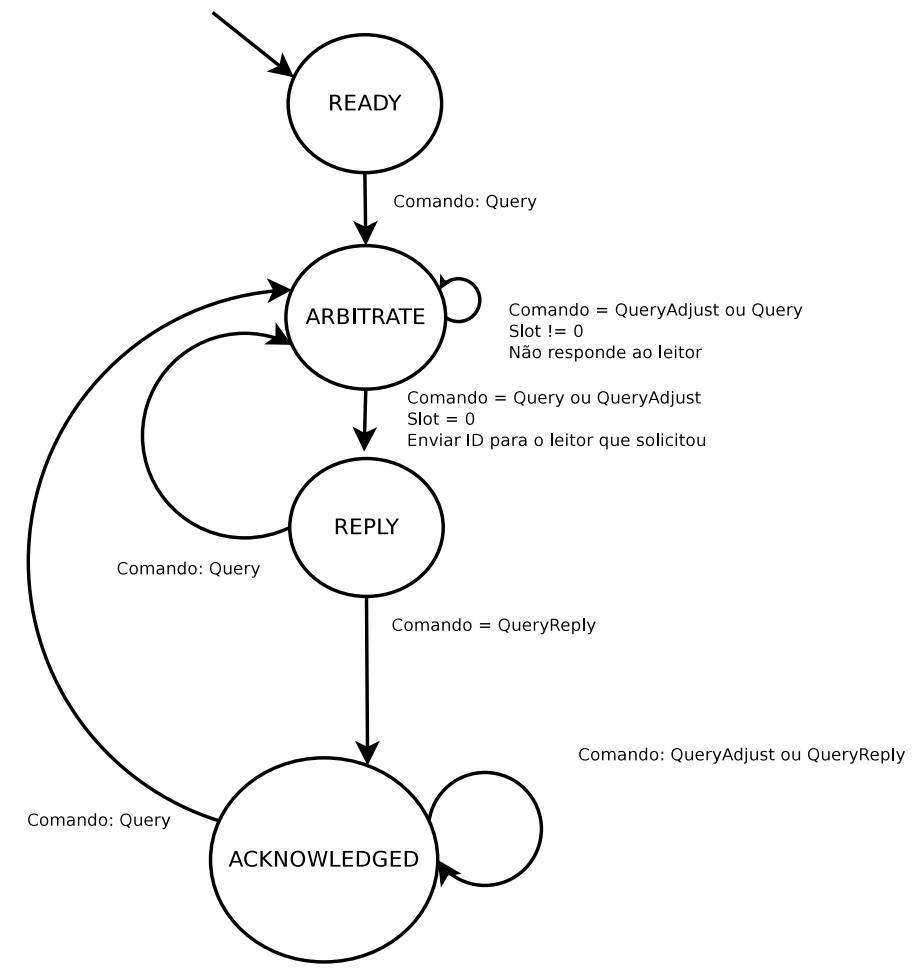

Figura 3.3: Diagrama de estados das etiquetas. Adaptado de EPCglobal, GS1 Inc. (2015)

$$
\begin{gathered}
Q_{f p}=\max \left(0, Q_{f p}-c\right) \\
Q=\operatorname{round}\left(Q_{f p}\right)
\end{gathered}
$$

O padrão definido em (EPCglobal, GS1 Inc., 2015) estabelece que o valor de $Q$ deve estar no intervalo $[0,15]$, justificando os limites das equações supracitadas. Posteriormente, em ambos os casos, o leitor envia um novo comando denominado QueryAdjust para que o tamanho do quadro seja ajustado (Algoritmo 2 - linhas 9 e 14) e o processo é reiniciado com um novo valor de $Q$, definido a partir da Equação 3.4 (Algoritmo 2 - linhas 13 e 18). Se apenas uma etiqueta responder ao leitor (Algoritmo 2 - linhas 16-20), a mesma é identificada através de um ACK com o comando QueryRep (Algoritmo 2 - linha 19), mudando para o estado ACKNOWLEDGED (Algoritmo 3 - linha 13) e as demais etiquetas decrementam em uma unidade seus números de slot gerados (Algoritmo 3 - linhas 15-17). O valor de $Q$ permanece inalterado (Algoritmo 2 - linha 17). O procedimento é reiniciado e termina quando o valor de $Q$ tornar-se igual a 0 (Algoritmo 2 - laço da linha 4) (Arjona et al., 2015; Cerciello et al., 2014). A Figura 3.3 ilustra o diagrama de estados das etiquetas baseado nos 
comandos recebidos e valor dos slots.

A Figura 3.4a detalha a troca completa de mensagens quando apenas uma etiqueta é identificada. O leitor envia inicialmente um dos três comandos (Query, QueryAdjust ou QueryRep) na mensagem 1. A etiqueta responde com um número aleatório denominado RNG16 em 2. Em 3 o leitor pede a confirmação para a etiqueta enviando um ACK com o RNG16 recebido. Se a confirmação do leitor estiver correta, a etiqueta envia seu identificador. Os demais passos correspondem a comandos avançados de LEITURA/ESCRITA, fora do escopo dos algoritmos anticolisão. Os Algoritmos 2 e 3 formalizam a versão simplificada deste procedimento, correspondente à Figura 3.4b, onde o leitor envia um comando de Query em 1, recebe o identificador da etiqueta em 2 e em 3 confirma o recebimento com um ACK. Como a troca de mensagens na identificação de uma única etiqueta não é levada em consideração para fins de avaliação de desempenho, os algoritmos supracitados utilizam a versão simplificada.

\subsubsection{Wang et al. (2009)}

Wang et al. (2009) apresentou uma proposta de um algoritmo $Q$ otimizado que determina os melhores valores de $Q$ de acordo com a quantidade aproximada de etiquetas restantes no processo de identificação, melhorando assim as métricas por eles definidas. A estimativa da quantidade de etiquetas não é definida, podendo ser utilizada alguma proposta da literatura como a apresentada por Kodialam e Nandagopal (2006). O algoritmo opera através da definição de um novo valor de $Q$ ao fim de cada identificação de uma etiqueta, aplicando a fórmula $Q=\log _{2} n$, onde $n$ representa a quantidade de etiquetas ainda não identificadas. O novo valor de $Q$ é informado às etiquetas que selecionam novamente um novo slot e o algoritmo $Q$ padrão é reiniciado. A proposta apresenta um pequeno melhoramento em relação ao Algoritmo Q (aproximadamente 5\% em relação a quantidade de etiquetas lidas por segundo) uma vez que a estratégia de atualização do valor de $Q$ é melhorada.

\subsubsection{Chen (2014)}

Chen (2014) propôs um novo algoritmo considerado de fácil implementação em dispositivos com baixos recursos de hardware aliado a uma eficiência do sistema (relação entre o 


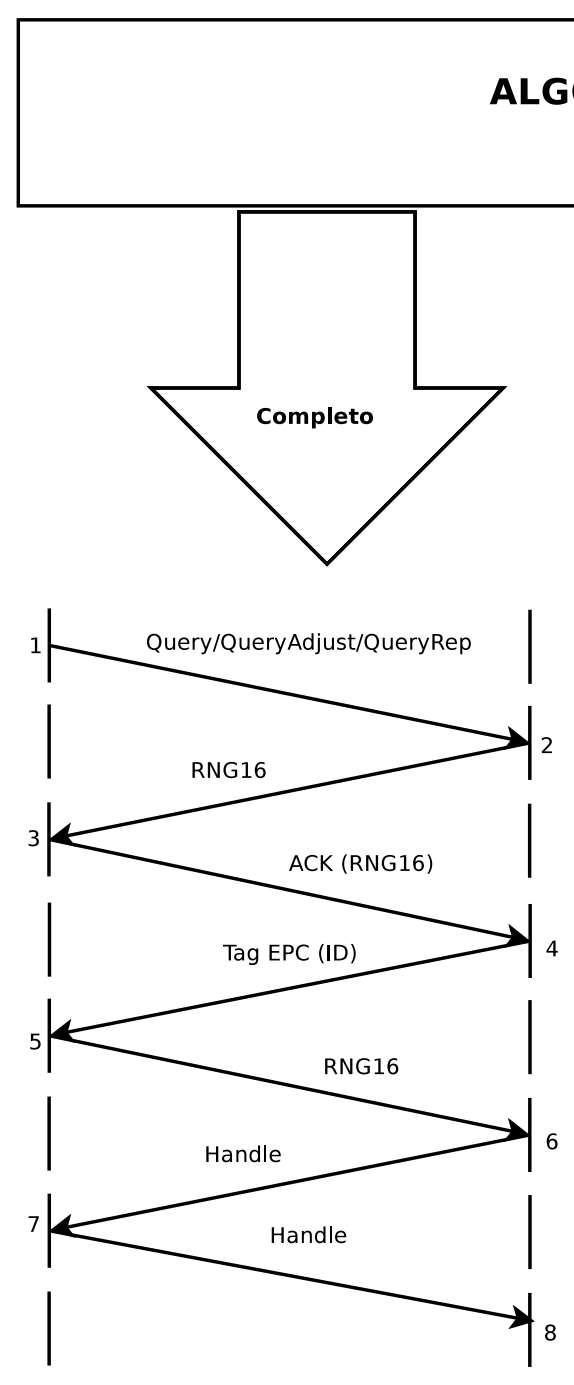

(a)

\section{ALGORITMO ANTI-COLISÃO Algoritmo Q}
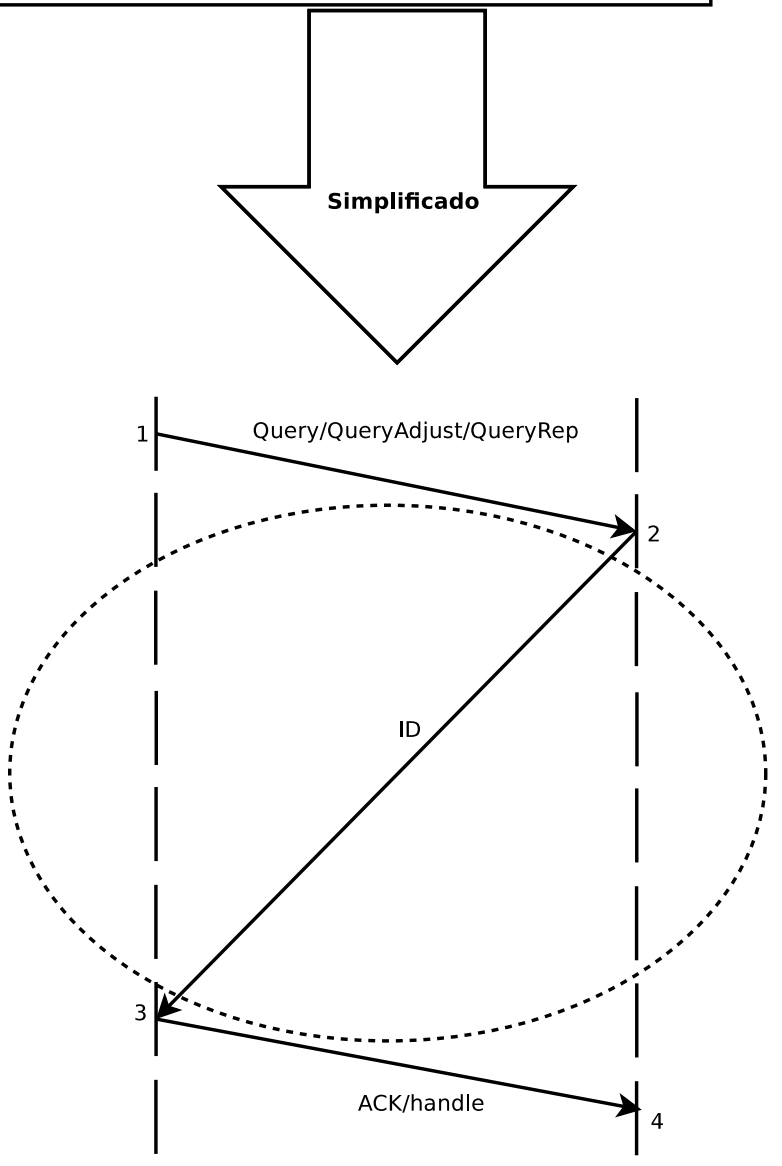

(b)

Figura 3.4: Troca de mensagens para identificar uma única etiqueta.

número de etiquetas e a quantidade total de slots utilizados, também chamada de normalized throughput) próxima ao máximo teórico de $0.368\left(e^{-1}\right)$ (Schoute, 1983). Assim como Wang et al. (2009), Chen (2014) atacou o problema da atualização correta do valor de $Q$. O fluxograma do mecanismo proposto está definido na Figura 3.5 (Chen, 2014).

O leitor inicia enviando um comando Query para todas as etiquetas (PASSO 1), que corresponde ao tamanho do quadro $2^{Q}$. Ao final do slot $i$, o leitor conta o número de slots vazios $E_{i}$, de sucesso $S_{i}$ e de colisão $C_{i}$. Depois o tamanho estimado da quantidade de etiquetas é calculado (PASSO 2) através da Equação 3.5.

$$
n^{\prime}=\left(S_{i}+k \times C_{i}\right) \times \frac{L}{i}
$$




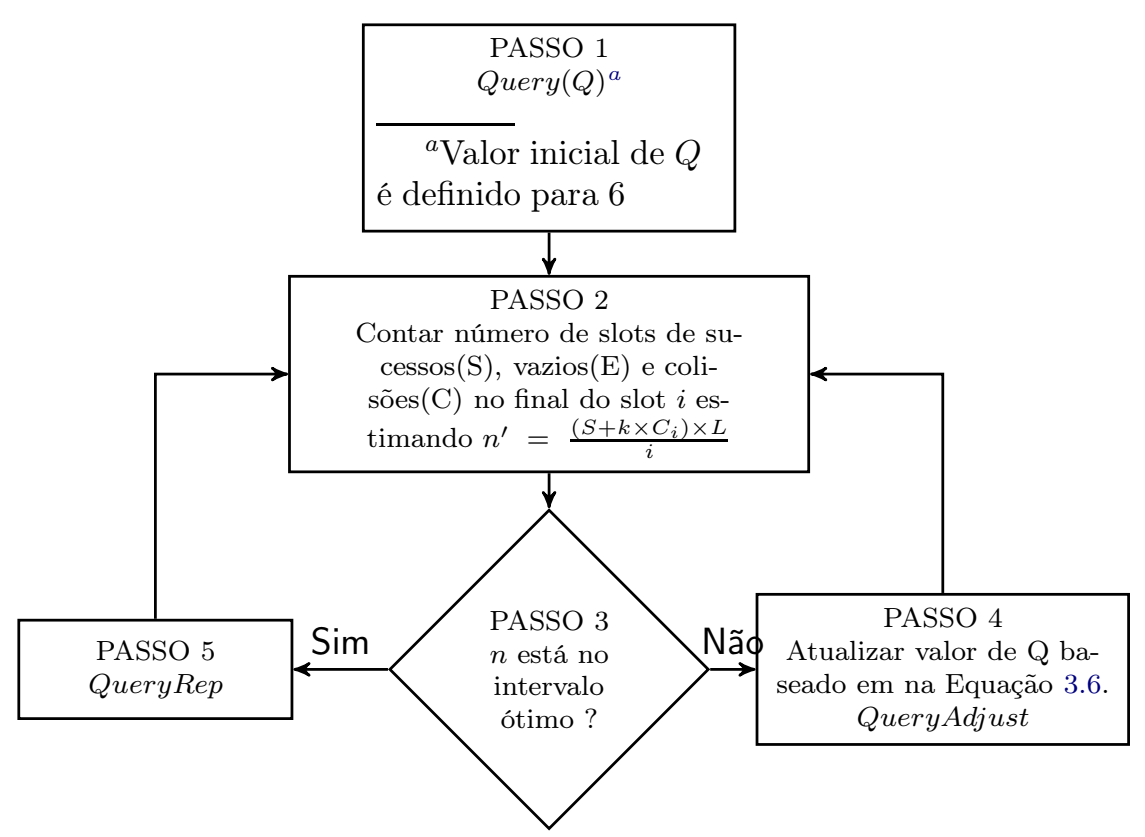

Figura 3.5: Fluxograma da proposta de Chen (2014).

$$
\text { backlog }=n^{\prime}-S_{i}
$$

Onde $k$ é um coeficiente e $L$ é o tamanho do quadro. Uma vez que pelo menos duas etiquetas estão envolvidas em uma colisão, o coeficiente $k$ pode ser definido como 2 , mas os autores utilizam 2,39, de acordo com Schoute (1983), que será detalhado na Seção 3.3. Comparado a outros métodos de estimação apresentados na Seção 3.3, o método de Chen (2014) é simples e fácil de implementar, pois envolve apenas operações aritméticas simples. O algoritmo segue verificando se o valor estimado está no intervalo ótimo (PASSO 3), ou seja, se o tamanho do quadro está adequado à quantidade estimada de etiquetas não identificadas $\left(n^{\prime}\right)$. Caso negativo, o valor de $Q$ será atualizado de acordo com a Equação 3.6 e as etiquetas selecionarão outro slot aleatório (PASSO 4). Caso positivo, o leitor chama o próximo slot (PASSO 5). A principal diferença deste algoritmo é a possibilidade de ajustar o tamanho do quadro antes do final do mesmo, o que leva à um melhoramento aproximado de $3 \%$ do desempenho em relação à métrica de vazão.

\subsubsection{Chen (2015)}

Chen (2015) propôs mais um algoritmo anti-colisão de simples implementação baseado na escolha do melhor tamanho de quadro $(a)$ em relação à razão entre a duração (em tempo) 
de um slot de colisão e um slot vazio, representado por $x$ na Equação 3.7.

$$
a=-0.0097 x^{2}+0.273 x+0.7707
$$

A proposta assim como o algoritmo anterior do mesmo autor (Chen, 2014), conta a ocorrência dos três tipos possíveis de slots no i-ésimo slot e calcula o melhor valor de $Q$ de acordo com a Equação 3.9. Onde $n$ representa a quantidade estimada de etiquetas, $S$ e $C$ o número de slots de sucesso e colisão respectivamente e $a$ o melhor valor baseado na razão entre a duração em tempo dos slots de colisão e vazio, valor calculado através da Equação 3.7.

$$
\begin{gathered}
n=\frac{(S+2.39 \times C) L}{i} \\
Q_{o p t}=\operatorname{round}\left(\log _{2}(a \times n)\right)
\end{gathered}
$$

Resultados de simulação mostraram que a métrica $C_{e f}$, eficiência de uso do canal, atinge valores próximos a $83 \%$, o que representa um ganho de $5 \%$ a $10 \%$ em relação a outras propostas DFSA típicas. Já a métrica $\operatorname{Tags}_{\text {sec }}$, métrica que mede a quantidade de etiquetas identificadas por segundo, atinge valores aproximados de 400 etiquetas por segundo, valor este que supera o valor de 300 que é o desejável por aplicações de logística e controle de estoques (Finkenzeller et al., 2010).

\subsection{DFSA Puro com estimação de backlog (PDFSA-b)}

Diversas propostas estão descritas na literatura acerca do cálculo do tamanho do próximo quadro (backlog) . O mais simples e chamado de limite inferior (Lower Bound - LB) calcula o tamanho do próximo quadro baseado na quantidade de slots de colisão $(\mathrm{col})$ utilizando a Equação 3.10. A equação surgiu a partir do principio básico de que se ocorreu uma colisão, pelo menos duas etiquetas colidiram, por isso é chamada de "limite inferior". Schoute (1983) , após extenso estudo analítico, chegou a conclusão de que um valor ideal para cálculo do backlog seria multiplicar o número de colisões pela constante 2,39 , sendo o backlog calculado 
a partir da Equação 3.11.

$$
\begin{gathered}
\text { backlog }=2 * \operatorname{col} \\
\text { backlog }=2.39 * \text { col }
\end{gathered}
$$

Eom e Lee (2010) propuseram uma fórmula interativa que calcula o backlog a partir do tamanho do quadro anterior (L), quantidade de colisões (col) e quantidade de sucessos (suc) (linhas 5 a 9 do Algoritmo 4). A Equação 3.14 calcula o backlog a partir das Equações 3.13 e 3.12, que encontram-se provadas analiticamente em (Eom e Lee, 2010). O Algoritmo 4 formaliza a função interativa para cálculo do backlog (laço das linhas 4 a 13). O critério de parada é definido como 0,01 como padrão, podendo ser modificado conforme desejado. O mesmo representa a diferença entre os dois últimos valores calculados pela Equação 3.13. O valor inicial de $y_{1}$ é 2 (linha 2), o que significa o número mínimo de etiquetas em colisão em determinado slot.

$$
\begin{gathered}
b_{\text {prox }}=\frac{\text { backlog }}{y_{1} * \operatorname{col}+\text { suc }} \\
y_{\text {prox }}=\frac{1-e^{\frac{-1}{b_{\text {prox }}}}}{b_{\text {prox }} *\left(1-\left(1+\frac{1}{\text { brox }}\right) * e^{\frac{-1}{b_{\text {prox }}}}\right)} \\
L=y_{\text {prox }} * \operatorname{col}
\end{gathered}
$$

Andrade e Goncalves (2013) propuseram modificações para otimizar a proposta de Vogt (2002) originando os algoritmos baseados em DFSA IV-I e IV-II (Improved Vogt I e Improved Vogt II). Os autores trabalharam otimizando os pontos onde o algoritmo Vogt original poderia ser melhorado, como nos casos em que todos os slots do quadro estejam em colisão. Assim como a proposta de Eom e Lee (2010), o algoritmo IV-I é iterativo, e possui desempenho equivalente ou um pouco superior. Já o algoritmo IV-II não possui características iterativas e seu desempenho é um pouco superior ao IV-I, superando em média Eom-Lee e Vogt. Por realizar muito processamento, devido a característica iterativa, ambos algoritmos 


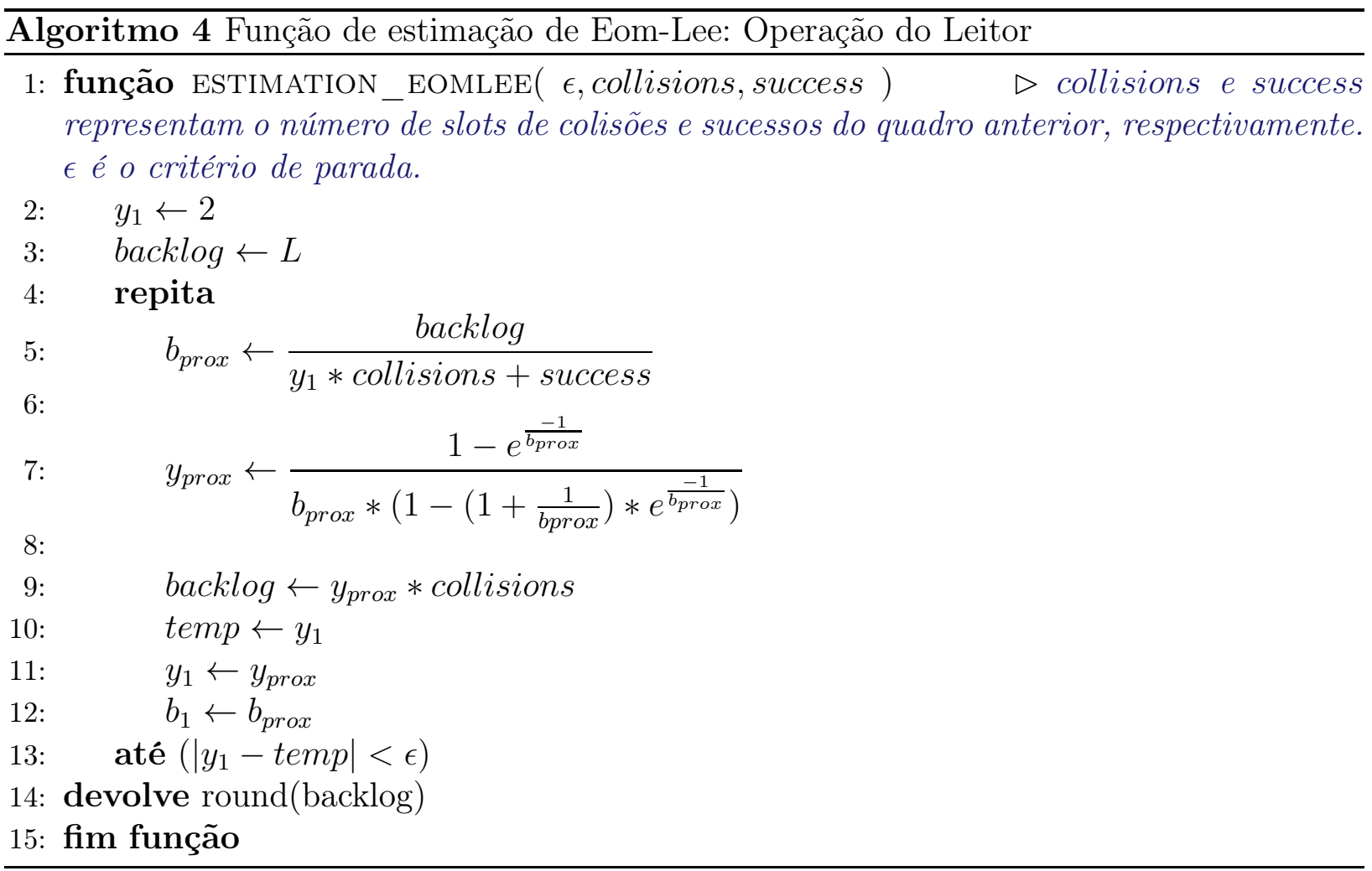

de Eom-Lee e IV-I podem ter a aplicação prática inviabilizada em leitores móveis, devido ao aumento do gasto de energia em comparação com propostas de implementação mais simples (Klair et al., 2009). As próximas subseções descrevem outras propostas importantes e relevantes para fins de comparação com os resultados desta tese.

\subsubsection{Zhu e Yum (2010)}

Zhu e Yum (2010) propuseram um método para cálculo de um backlog ótimo com o objetivo de melhorar o desempenho geral do processo de identificação das etiquetas. Os autores modelaram o processo de leitura como uma cadeia de Markov. Os tamanhos de quadros podem ser calculados analiticamente ou através de um procedimento recursivo. Apesar dos ganhos obtidos, cerca de $6 \%$ de aumento de vazão, o mesmo não é indicado para aplicações reais, conforme afirmação dos próprios autores, porque a proposta requer grande poder computacional e de armazenamento, assim como o conhecimento prévio da quantidade de etiquetas. 


\subsubsection{Cui e Zhao (2010)}

Cui e Zhao (2010) propuseram um algoritmo com o objetivo de fornecer uma estimação confiável da quantidade de etiquetas no menor tempo possível. Eles resolveram o seguinte problema: Dado um erro $\epsilon$ e uma probabilidade $p$ para um conjunto de $n$ etiquetas, como obter uma estimação $n^{\prime}$ tal que $\left|\left(n-n^{\prime}\right) / n<\epsilon\right|$ com probabilidade maior que $p$. A proposta melhora o algoritmo de Kodialam e Nandagopal (2006) e é chamado de Fast Zero Estimiation - (FZE). A Figura 3.6 ilustra o funcionamento do algoritmo como um fluxograma.

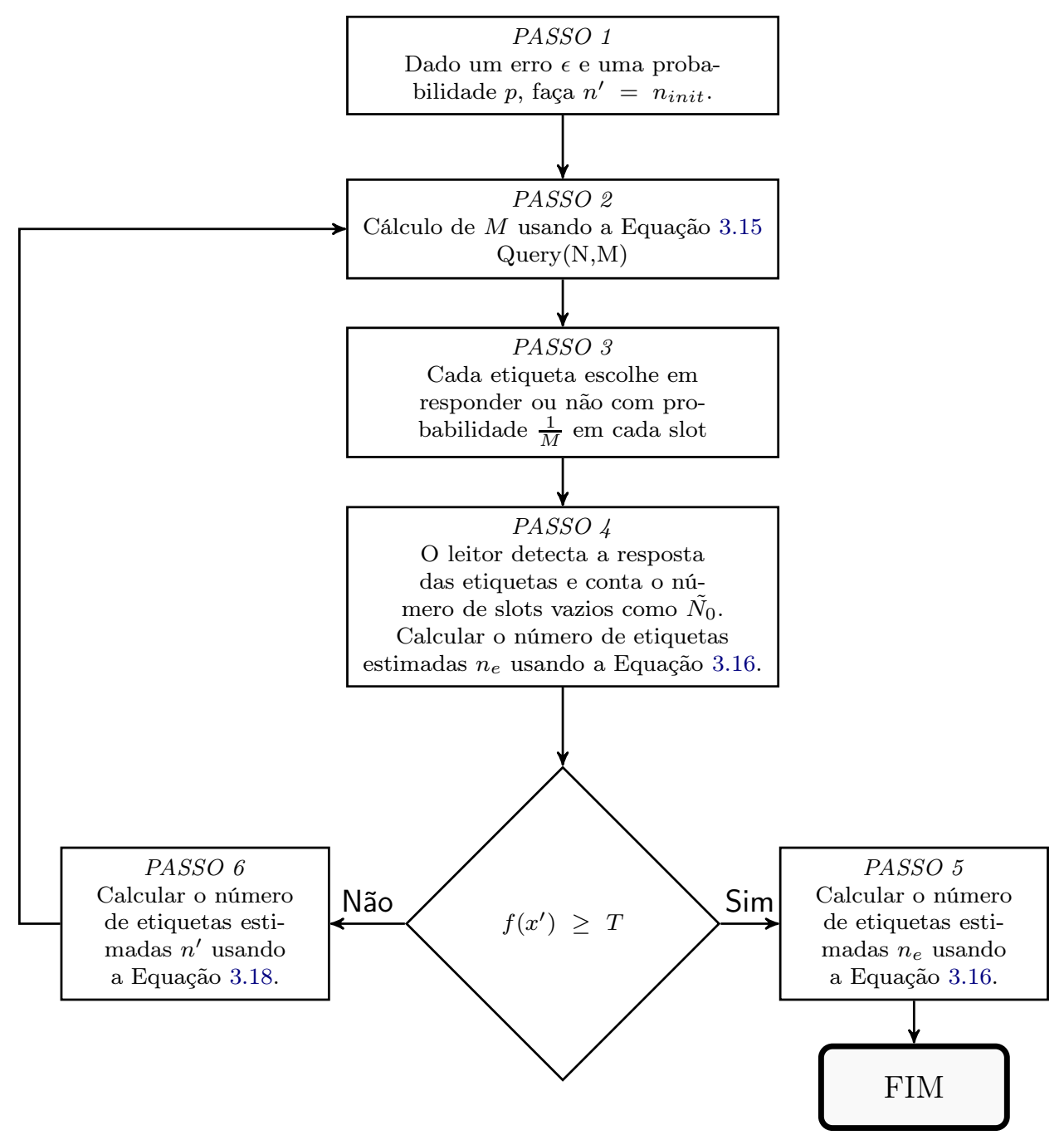

Figura 3.6: Fluxograma do FZE (Cui e Zhao, 2010).

O algoritmo inicia definindo o tamanho inicial de quadro $n_{\text {init }}(\mathrm{PASSO} 1)$. Posteriormente o valor de $M$ é calculado (Equação 3.15), a probabilidade de resposta das etiquetas é enviada com o valor $N^{1}$ para todas as etiquetas (PASSO 2). Ao receberem a requisição, cada etiqueta escolhe responder ou não com uma probabilidade $\frac{1}{M}$ em cada slot (PASSO 3). O leitor detecta

\footnotetext{
${ }^{1}$ Tamanho atual do quadro
} 
as respostas das etiquetas e conta o número de slots vazios como $\tilde{N}_{0}$. O leitor então calcula o valor estimado do número de etiquetas conforme Equação 3.16 (PASSO 4). Se $x^{\prime}=\frac{N}{\tilde{N}_{0}}$ satisfaz a inequação $f\left(x^{\prime}\right) \geq T$ (ver Equação 3.17) significa que o resultado é confiável e então o leitor utiliza $n_{e}$ como o valor estimado do número de etiquetas e o processo termina (PASSO 5). Caso contrário, $n^{\prime}$ é calculado de acordo com a Equação 3.18 (PASSO 6) e o leitor inicia um novo ciclo de leitura, voltando ao PASSO 2. Apesar do algoritmo proposto reduzir o tempo de estimação em torno de $66 \%$ em relação ao Algoritmo Q, pois o mesmo utiliza slots de tempo muito curtos, o mesmo não é adequado para a maioria das aplicações práticas, onde o prévio conhecimento do número de etiquetas é desconhecido.

$$
\begin{gathered}
M=\frac{1}{1-\sqrt[n^{\prime}]{\frac{1}{\widehat{x}}}} \\
n_{e}=\frac{\log \frac{\tilde{N}_{0}}{N}}{\log \left(1-\frac{1}{M}\right)} \\
f(x)=\frac{\log x}{\sqrt{x-1}} \\
n^{\prime}= \begin{cases}16 n^{\prime}, & \text { if } n_{e}=\infty \\
n_{e}, & \text { caso contrário }\end{cases}
\end{gathered}
$$

\subsubsection{Tong et al. (2012)}

Tong et al. (2012) propuseram um algoritmo chamado de Bayesian Estimation DFSA Anti-Collision Algorithm (BEAC) para sistemas RFID. A abordagem faz uso não apenas dos dados do quadro atual mas também dos quadros anteriores, uma vez que é realizado um ajuste dinâmico do tamanho dos quadros baseado na teoria Bayesiana. Resultados de simulação mostraram que a estratégia pode estimar a quantidade de etiquetas de forma mais precisa, melhorando o desempenho geral da identificação, quando comparado com outras propostas da literatura como Schoute (1983) e Vogt (2002).

O algoritmo, ilustrado no fluxograma da Figura 3.7, inicia através da determinação do tamanho inicial do quadro com 64 slots(PASSO 1). Após a leitura de todos os slots do 
primeiro quadro, o leitor inicia a distribuição de probabilidades (PASSO 2) definindo o limite inferior para $(S+2 \times C)^{2}$. Já o limite superior é definido como o valor máximo de $n$ que faz $P(E \cap S) \geqslant\left(10^{-5}\right)^{3}$. Se nenhuma colisão é detectada após o primeiro quadro o algoritmo termina. Caso contrário o leitor deve coletar a quantidade de cada tipo de slots (PASSO 3) do quadro atual para estimar o número de etiquetas. Isto é feito pelo cálculo da probabilidade $P(n \mid I)$ onde $I$ denota a evidência de $E, S, C$, de acordo com a teoria Bayesiana. Finalmente, no PASSO 4, o tamanho do próximo quadro é ajustado de acordo com a Equação 3.19, onde $P_{t+1}(n-S)$ representa a distribuição de probabilidades inicial da quantidade de etiquetas no começo do quadro $(t+1)$. Os ganhos em desempenho da métrica vazão, com relação ao Algoritmo $Q$, atingem valores próximos a $3 \%$.

$$
E_{t+1}(n)=\frac{\sum_{n_{\min }}^{n_{\max }}(n-S) \times P_{t+1}(n-S)}{\sum_{n_{\min }}^{n_{\max }} P_{t+1}(n-S)}
$$

\subsubsection{Shakiba et al. (2014)}

Shakiba et al. (2014) propuseram uma nova forma de estimação da quantidade de etiquetas baseada na teoria de probabilidades do paradoxo de aniversários. Na teoria da probabilidade, o problema do paradoxo de aniversários procura pela probabilidade de que ao menos duas pessoas façam aniversário dentro de um universo de $n$ pessoas. Esta teoria assume que cada dia do ano possui igual probabilidade de aniversário e que todos os aniversários são independentes. Substituindo as pessoas pelas etiquetas e os dias pelos slots os autores estimaram a quantidade de etiquetas de acordo com a Equação 3.22. A Figura 3.8 resume a proposta através de um fluxograma.

Os resultados indicam alta precisão e pequeno erro de estimação, fazendo com que a utilização dos recursos de rede seja mais eficiente. A estimação do backlog utiliza os valores calculados nas Equações 3.20 e 3.21 para estimar a quantidade de etiquetas não identificadas com a Equação 3.22, onde $C_{k}$ corresponde ao número de slots de colisão, $C_{1}$ o número de

\footnotetext{
${ }^{2} S$ : quantidade de slots de sucesso e $C$ de colisão

${ }^{3} E$ : quantidade de slots vazios
} 


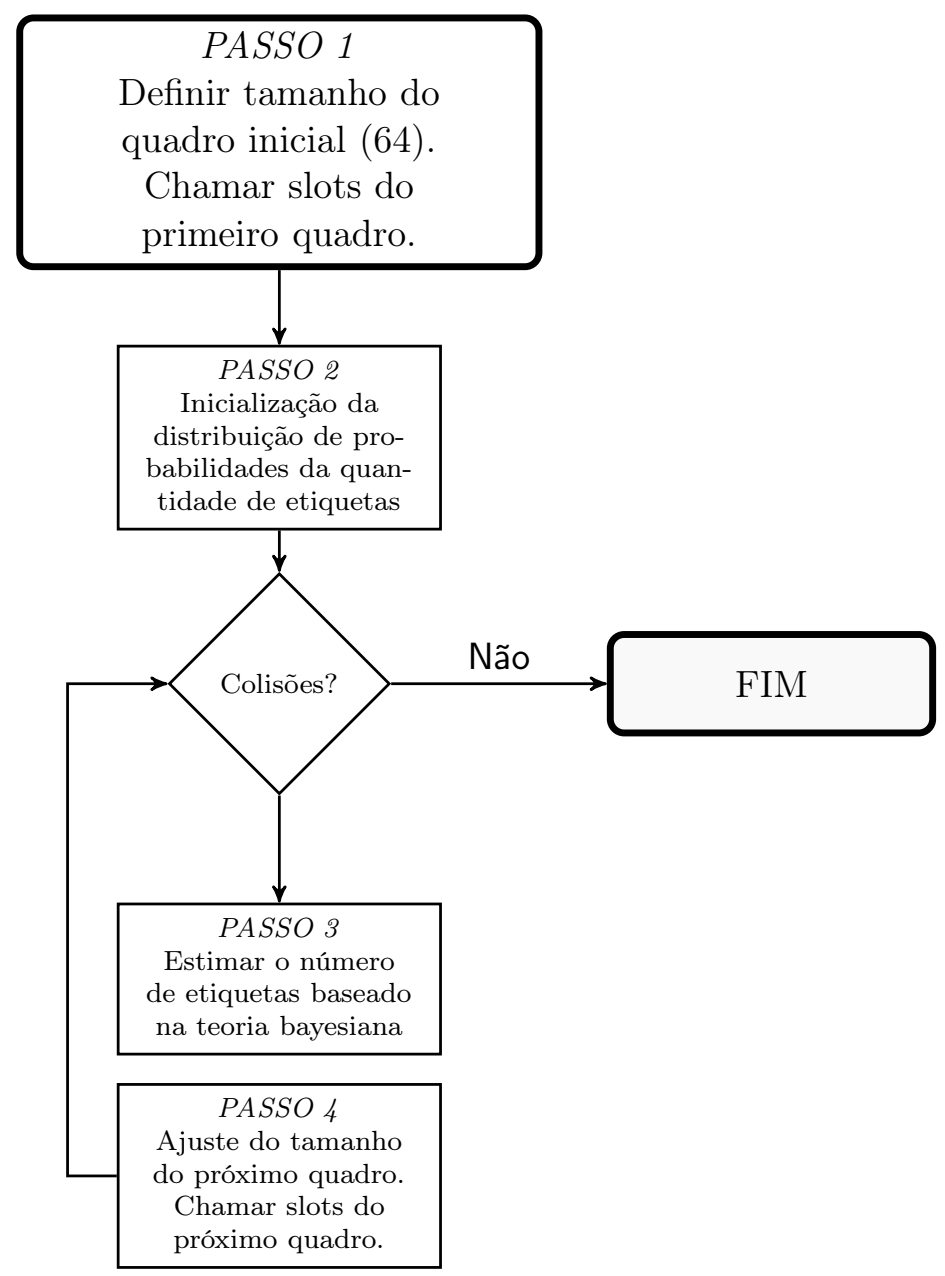

Figura 3.7: Fluxograma da proposta de Tong et al. (2012).

slots de sucesso e $N$ é o tamanho do quadro atual. O valor $n_{\text {est }}$ corresponde ao backlog que será utilizado para ajustar o tamanho do próximo quadro.

$$
\begin{gathered}
E_{n}=C_{k}+C_{1} \\
\alpha=\frac{N-1}{N} \\
n_{e s t}=\frac{\log \left(1-(1-\alpha) E_{n}\right)}{\log \alpha}
\end{gathered}
$$

O algoritmo opera seguindo o padrão de funcionamento do mecanismo DFSA-b utilizando a Equação 3.22 ao calcular o tamanho do próximo quadro. Resultados de simulação mostraram que a proposta reduz a quantidade total de slots utilizados em aproximadamente 10,99\%, 17,45\%, e 87,74\% em comparação com os mecanismos Lower Bound (Vogt, 


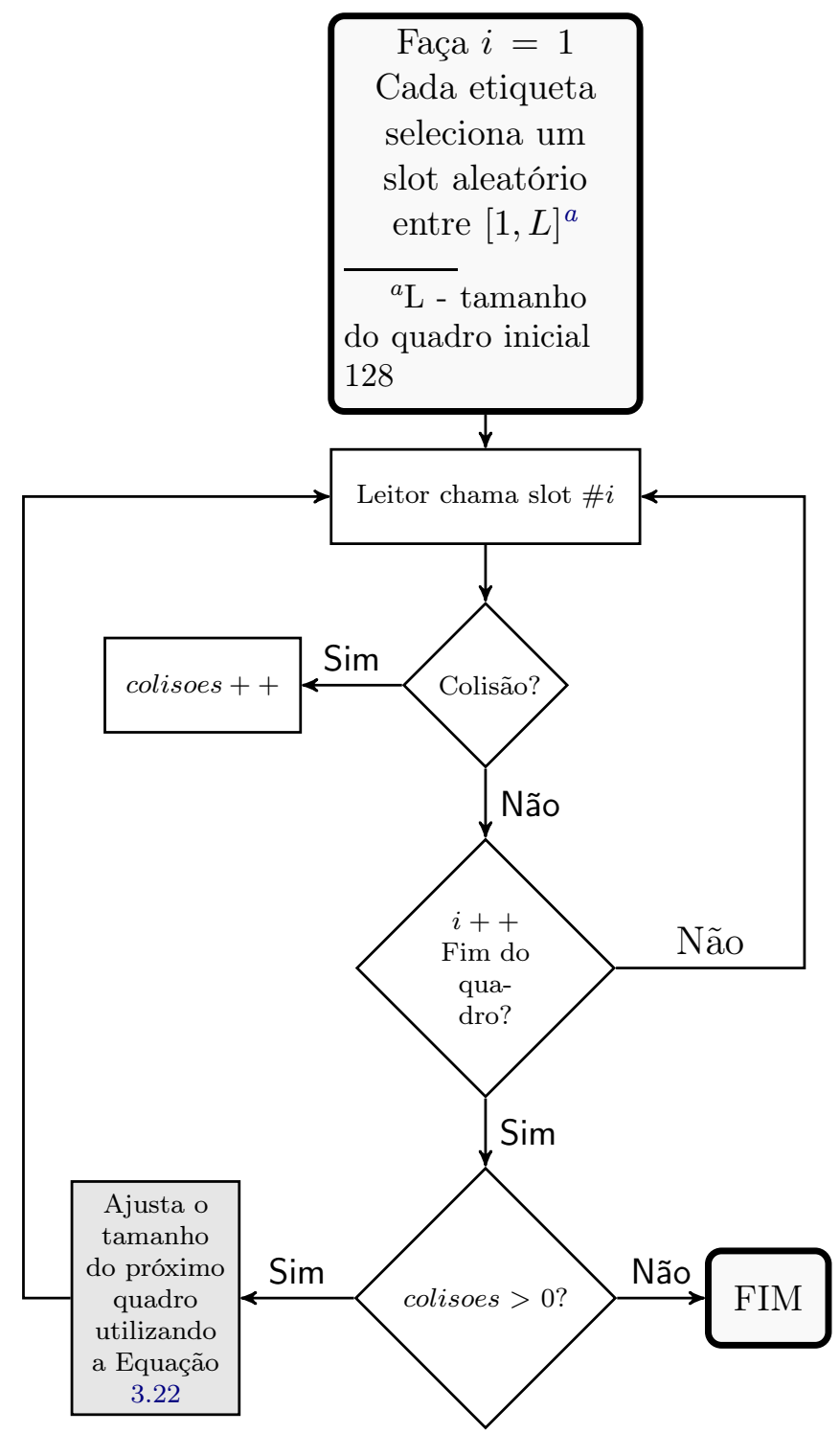

Figura 3.8: Fluxograma da teoria do paradoxo de aniversários.

2002), Schoute(Schoute, 1983), and Vogt(Vogt, 2002), respectivamente, quando tem-se 1000 etiquetas.

\subsubsection{Yao et al. (2014)}

Yao et al. (2014) propuseram um protocolo de identificação de etiquetas, denominado FAST. O fluxograma resumido de funcionamento do protocolo é mostrado na Figura 3.9.

O protocolo inclui um mecanismo de tolerância a colisões (Collision Tolerant Mechanism - CTM) que identifica o número de etiquetas nos slots de colisão. Quando a etiqueta seleciona um slot a mesma envia uma assinatura aleatória conhecida. Quando mais de 1 etiqueta escolhem o mesmo slot e transmitem suas assinaturas ao mesmo tempo, uma colisão 


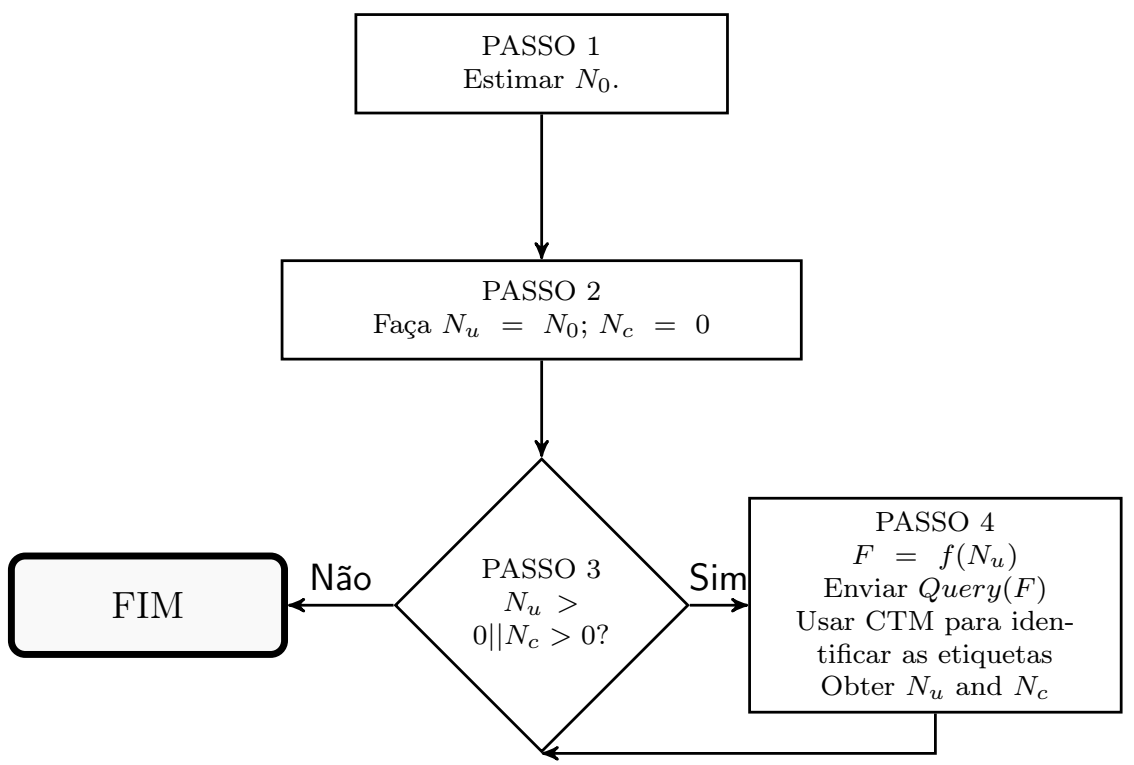

Figura 3.9: Fluxograma da proposta de Yao et al. (2014).

é detectada. Ao identificar a colisão, o leitor inicia a detecção de todas as assinaturas das respostas recebidas através de um processo de detecção de assinaturas utilizando correlação cruzada, através da qual as assinaturas podem ser detectadas corretamente, mesmo quando há interferência da colisão. Então o leitor solicita que as etiquetas enviem seus identificadores sequencialmente através de uma série de comandos de confirmação, nos quais cada assinatura é utilizada como parâmetro, permitindo que cada etiqueta identifique se a requisição é destinada a ela. Este mecanismo permite que o leitor identifique todas as etiquetas envolvidas no determinado slot.

O leitor inicia fazendo uma estimação da quantidade de etiquetas presentes em sua área de cobertura. denotado como $N_{0}$ (PASSO 1), e considera o número de etiquetas não identificadas como $N_{u}=N_{0}$ (PASSO 2); em outras palavras, ele inicializa a contagem do número de slots de colisão como $N_{c}=0$. Então ele inicia uma série de quadros até ambos $N_{u}=0$ e $N_{c}=0$. Como $N_{u}$ pode não ser a quantidade atual de etiquetas não identificadas, ele adicionalmente checa se $N_{c}=0$ (PASSO 3) para finalizar o processo de identificação. Em cada quadro, o leitor primeiro determina o tamanho de quadro, denominado de $F$, utilizando a função $f\left(N_{u}\right)$ (PASSO 4). O leitor então envia um comando $Q u e r y(F)$ e utiliza o procedimento $C T M$ para identificar as etiquetas. Ambos $N_{u}$ e $N_{c}$ são ajustados dinamicamente no final de cada quadro, de acordo com as informações obtidas dos slots de colisão do quadro atual (PASSO 4). Resultados de simulação mostraram que o mecanismo pode melhorar o desempenho em 
relação a quantidade de etiquetas lidas por segundo em até $25 \%$ com relação ao Algoritmo Q.

\subsubsection{Lin et al. (2015)}

Lin et al. (2015) propuseram o mecanismo denominado Prefix Assisted Frame Slotted Aloha Approach (PA-FSA), com o objetivo de desenvolver uma forma mais precisa e rápida de estimar a quantidade de etiquetas não identificadas em relação aos mecanismos existentes. Ao responder uma solicitação do leitor, cada etiqueta anexa um prefixo ao parâmetro "RN16" (número aleatório de 16 bits) de resposta. No prefixo, cada etiqueta independentemente e aleatoriamente seleciona um bit e faz o bit correspondente como ativo (valor 1). Através da sincronização dos prefixos de múltiplas respostas e contando o número de bits ativos nos prefixos sobrepostos, o leitor pode prever quantas etiquetas sob sua cobertura estão respondendo em determinado momento, melhorando significativamente a precisão da estimação mesmo quando o tamanho da população está fora do alcance de algoritmos tradicionais FSA. Em resumo, o algoritmo proposto calcula a estimação resolvendo um problema de otimização definido na Equação 3.23.

$$
N_{\text {est }}=\arg \min _{N_{t}}\left\{\Delta\left(N_{t} \mid N_{t} \in\left[\left(N_{t}\right)_{l b}\right]\right),+\infty\right\}
$$

onde $N_{\text {est }}$ representa o valor estimado da quantidade de etiquetas, $N_{t}$ corresponde ao palpite do valor da quantidade total de etiquetas e $\left(N_{t}\right)_{l b}$ é o limite inferior de $N_{t}$. Como o nível de complexidade da proposta não é aplicável a implementações práticas, não replicou-se nesta tese os algoritmos e equações do protocolo, que podem ser encontrados em (Lin et al. , 2015). Resultados de simulação mostraram que a proposta atinge ganhos de até $14 \%$ em relação ao Algoritmo Q com relação a métrica de eficiência de uso do canal.

\subsection{Binary Tree Slotted Aloha}

Algoritmos da categoria BTSA possuem o princípio de criar quadros com dois slots ao se depararem com slots de colisão no quadro principal. As subseções a seguir detalham as 
principais propostas da literatura.

\subsubsection{Cui e Zhao (2009)}

Cui e Zhao (2009) melhoraram o Algoritmo Q através de ajustes no funcionamento do mesmo aliados a uma função que faz uma estimativa razoável do valor inicial de $Q$. A proposta é chamada de Parâmetro Q Modificado - PQM (Modified Q Parameter). A Figura 3.10 ilustra o fluxograma do algoritmo de estimação proposto.

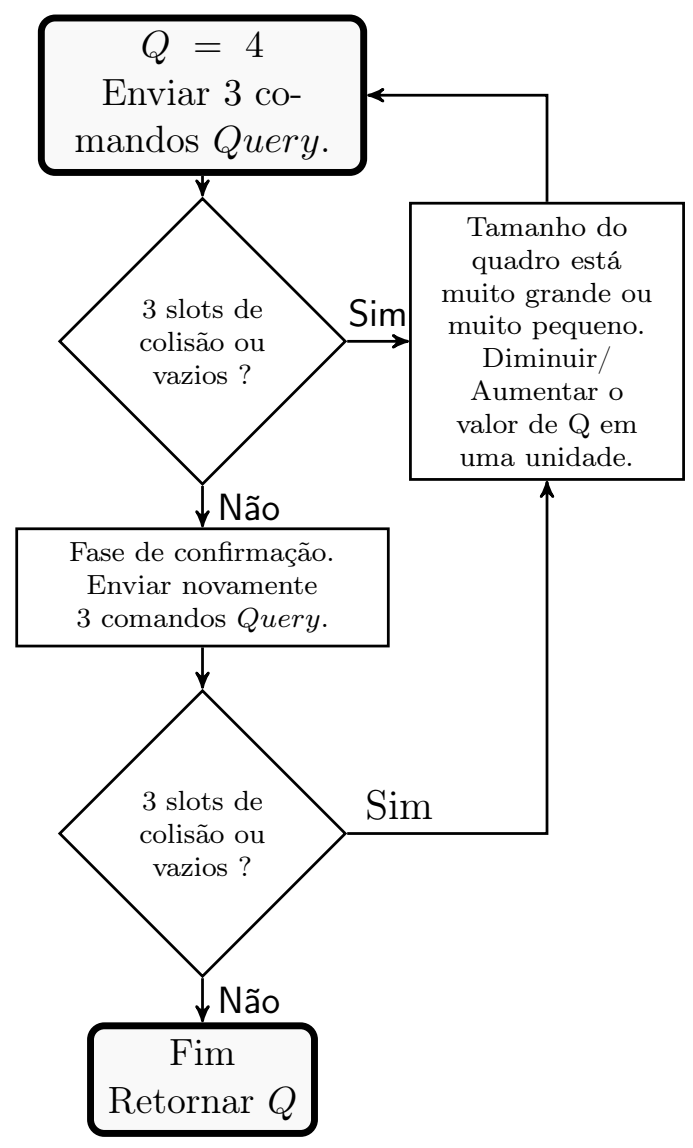

Figura 3.10: Fluxograma do procedimento de estimação de Cui e Zhao (2009).

O procedimento de estimação inicia enviando três comandos Query para as etiquetas. Após cada comando o leitor identifica o tipo de resposta recebido: sucesso, colisão ou vazio. Caso todos os comandos gerem colisão, o leitor deve aumentar em uma unidade o valor de $Q$, pois $Q$ é provavelmente um valor pequeno para a quantidade de etiquetas. Já se todos os comandos gerarem slots vazios o valor de $Q$ é decrementado em uma unidade, pois o valor de $Q$ é provavelmente alto. Em ambos os casos, o processo é reiniciado após o incremento ou decremento de $Q$. Caso contrário, o procedimento entra na fase de confirmação. Esta fase 
visa comprovar que o tamanho de $Q$ está adequado. O leitor envia novamente três comandos Query. Caso o número de slots de colisão e vazio sejam diferente de três o procedimento retorna o valor de $Q$ atual gravado em FinalQ e termina. Caso contrário o valor de $Q$ é novamente modificado (incremento ou decremento) e o procedimento volta ao início. Uma completa análise matemática levou o autor a chegar no valor três, ou seja, enviar três vezes o mesmo comando e confirmar novamente com três vezes o mesmo comando.

Após a estimação do valor inicial de $Q$, o algoritmo anticolisão proposto é iniciado, conforme mostra a Figura 3.11.

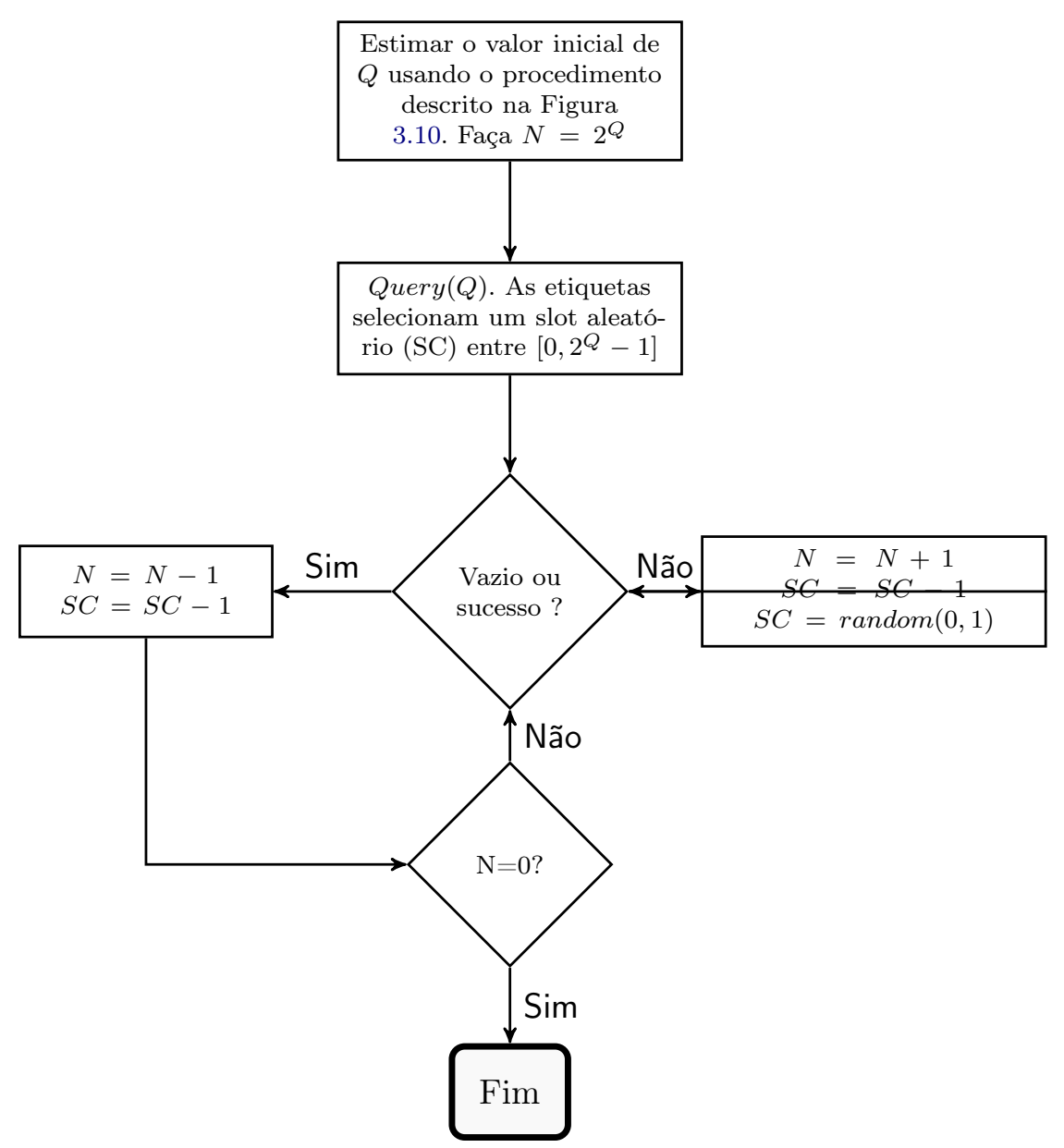

Figura 3.11: Fluxograma da proposta de Cui e Zhao (2009).

O leitor grava o valor $2^{Q}$ em $N$ e envia o comando inicial Query com o valor inicial de Q. Caso apenas uma ou nenhuma etiqueta responda então o parâmetro N é decrementado em uma unidade e um comando QueryRep é enviado, fazendo com que todas as etiquetas decrementem seus slots em uma unidade. O procedimento é encerrado quando $N$ for igual a 0. Ocorrendo novamente a situação de uma ou nenhuma resposta das etiquetas repete-se 
os passos anteriores. No caso de colisão, o valor de $N$ é incrementado em uma unidade, um novo comando QueryRep é enviado, fazendo com que as etiquetas em colisão selecionem um novo número aleatório de acordo com a Equação 3.24 e as demais decrementem seus slots em uma unidade. Este tratamento para as colisões resulta na separação das etiquetas em colisão em dois diferentes conjuntos reduzindo significativamente a probabilidade de uma nova colisão futura, de acordo com o autor. A proposta eleva o desempenho em aproximadamente $18 \%$ com relação à métrica vazão em comparação ao Algoritmo Q, conforme resultados de simulação.

$$
S C=\operatorname{random}(0,1)
$$

\subsubsection{Wu et al. (2013)}

Proposto em 2013, o BTSA (Binary Tree Slotted Aloha) de Wu et al. (2013) modifica o Algoritmo Q originando três algoritmos denominados de BTSA Dinâmico (BTSA-D) (Dynamic BTSA), BTSA Adaptativo (BTSA-A) (Adaptative BTSA) e BTSA Dividido (Splitting BTSA) (BTSA-S). As duas primeiras propostas (BTSA Dinâmico e BTSA Adaptativo) são baseadas no Algoritmo Q padrão definido em (EPCglobal, GS1 Inc., 2015), no entanto introduzem várias mudanças que elevam significativamente o desempenho em relação ao original. O BTSA-D, formalizado pelo Algoritmo 5, inicia definindo o valor de $Q$ inicial com o valor 4, 0 (linha 1).

O leitor então envia um comando QueryAdjust com o valor de $Q$ (linha 2). Caso nenhuma etiqueta ou mais de uma responda então o valor de $Q$ é modificado de acordo com as Equações 3.25 e 3.26 respectivamente (linhas 5-11).

$$
\begin{aligned}
& Q=\max (Q-1,0) \\
& Q=\min (Q+1,15)
\end{aligned}
$$

O leitor volta a enviar um novo comando QueryAdjust com o novo valor de $Q$ (linhas 8 e 11). Caso contrário, se apenas uma etiqueta responder (linha 12), o valor de $Q$ perma- 


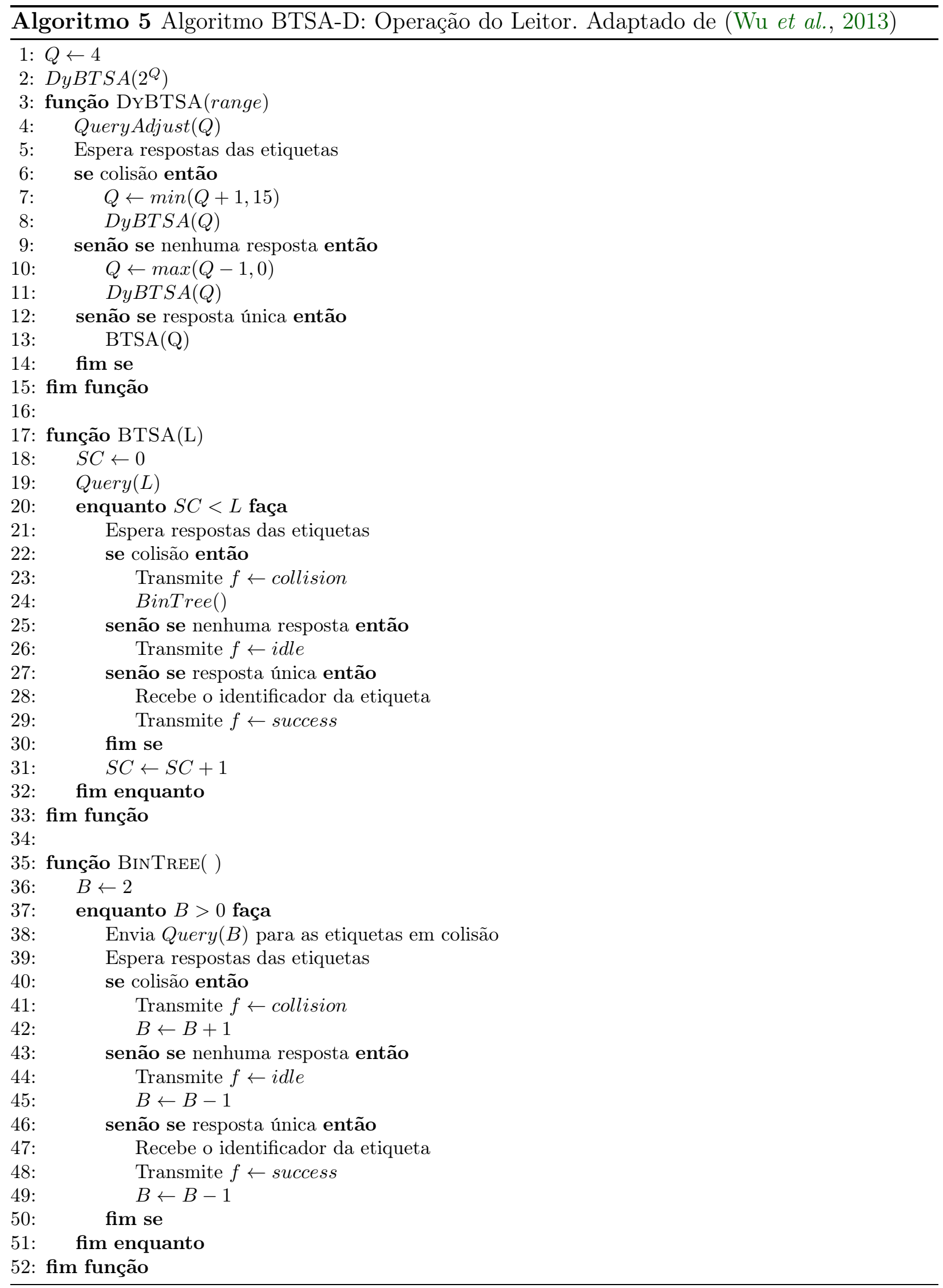

nece inalterado e o leitor chama o procedimento BTSA (linha 13) que inicialmente envia um comando Query (linha 18) com o valor atual de $L$ (que recebe o $Q$ atual) (linha 17). 
Posteriormente uma variável para a contagem de slots denominada SC (Slot Counter) é inicializada com valor 0 (zero) (linha 18). O leitor então espera as respostas das etiquetas (linha 21). Caso apenas uma etiqueta responda (linhas 27-30), o leitor envia um ACK identificando esta etiqueta (linhas 28-29). Se nenhuma etiqueta responder (linha 25), o leitor informa que o slot foi vazio (linha 26). Caso contrário, o leitor chama o método BinTree (linha 24) que identificará todas as etiquetas em colisão antes de continuar o procedimento BTSA. O algoritmo BinTree funciona basicamente como o Algoritmo Q padrão com as diferenças do $Q$ inicial tendo valor 2 (linha 36) e $c$ igual a 1. Quando todas as etiquetas em colisão são identificadas, continua-se o procedimento BTSA que vai para a próxima repetição do laço (laço das linhas 20-32), terminando quando o contador $S C$ for igual a $L$ (linha 20).

A Figura 3.12 mostra o Fluxograma de funcionamento do BTSA-D. Apesar de possuir desempenho superior ao Algoritmo Q, a implementação prática do BTSA-D pode ser prejudicada devido a grande quantidade de alterações que necessitam ser realizadas nos leitores e etiquetas em relação à algoritmos tradicionais como DFSA baseados em Schoute (1983), Eom e Lee (2010) e baseado no padrão definido em EPCglobal, GS1 Inc. (2015). As alterações são necessárias porque o Algoritmo Q pertence a categoria DFSA baseado em potência de 2, que é significativamente diferente de um BTSA.

O Algoritmo denominado BTSA-A possui funcionamento simular ao Algoritmo Q padrão com uma pequena alteração no lado da operação do leitor, conforme mostrado na Figura 3.13. Na situação em que ocorre um slot de colisão, as etiquetas nesta situação são imediatamente identificadas através do mesmo procedimento BinTree proposto em BTSA-D. As demais operações são iguais ao padrão original. A principal vantagem do BTSA-A para o BTSA-D está relacionada à quantidade de modificações a serem realizadas na operação do leitor, particularmente na situação onde ocorrem colisões. O desempenho é equivalente ao BTSA-D (Wu et al., 2013). Detalhes de implementação e funcionamento do BTSA-S podem ser encontrados em (Wu et al., 2013) devido ao mesmo ser significativamente diferente dos demais e utilizar técnicas diferentes do DFSA, foco desta tese. 


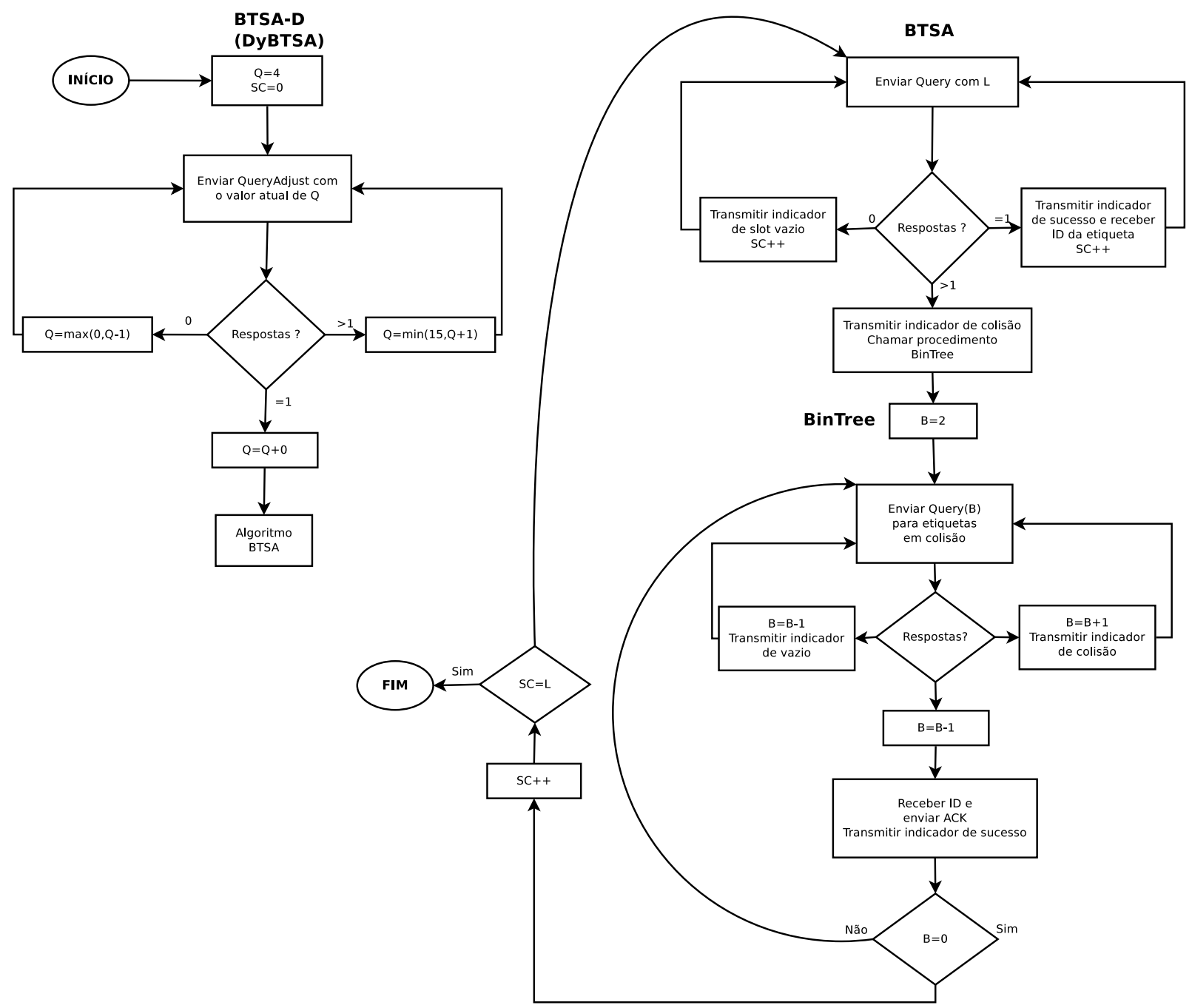

Figura 3.12: Fluxograma do Algoritmo BTSA-D. Baseado em Wu et al. (2013).

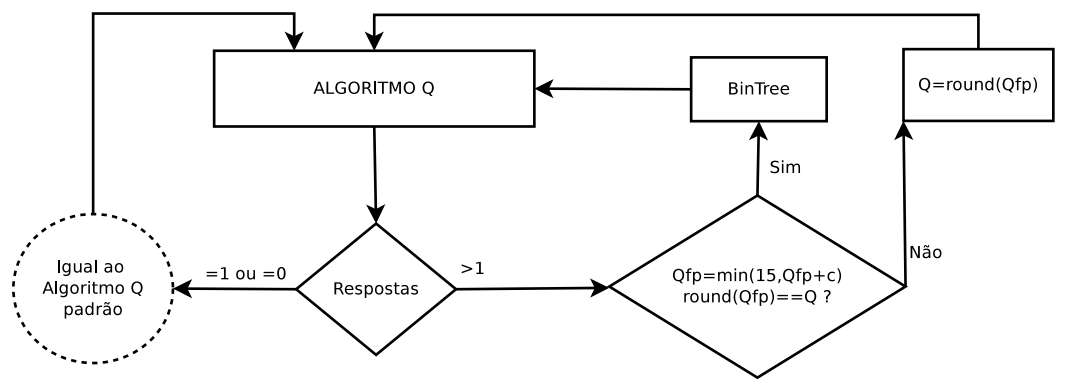

Figura 3.13: Fluxograma do Algoritmo BTSA-A. Adaptado de Wu et al. (2013).

\subsection{Híbridos}

Algoritmos anticolisão híbridos mesclam técnicas de outras categorias como PDFSA-b, DFSA-2 e BTSA em alguma fase de seu funcionamento. Em geral buscam obter o melhor de cada técnica. 


\subsubsection{He e Wang (2013)}

He e Wang (2013) propuseram um algoritmo anticolisão híbrido para sistemas RFID passivos. A proposta possui dois objetivos: 1) eliminar os slots vazios de um quadro; 2) processar imediatamente as colisões que são detectadas em determinado slot através da alocação de novos slots no final do quadro. Assume-se que o tamanho do quadro é aproximadamente igual ao número de etiquetas. Este fato não acontece na maioria dos cenários práticos. O fluxograma da Figura 3.14 resume o funcionamento do algoritmo.

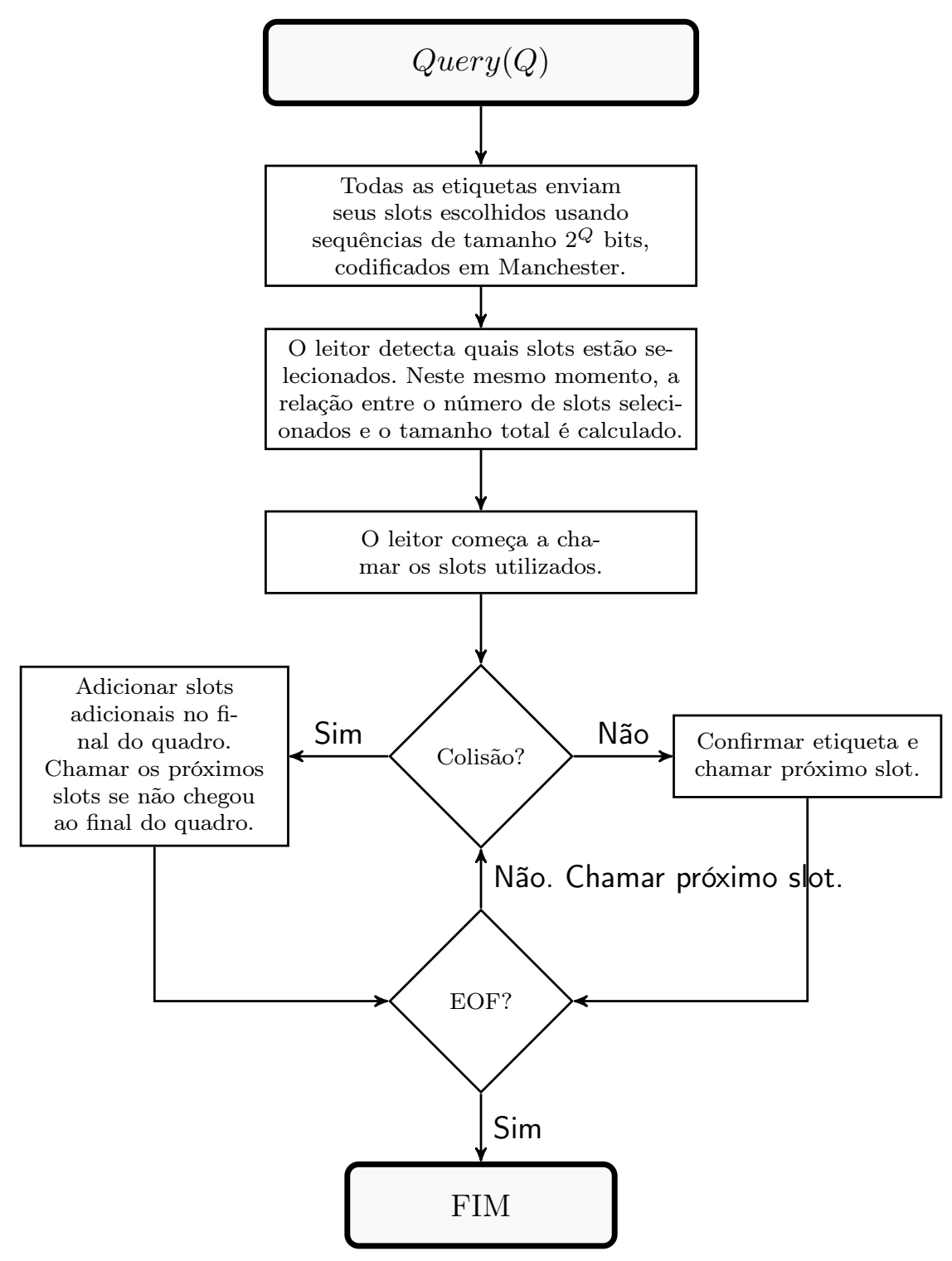

Figura 3.14: Fluxograma da proposta de He e Wang (2013).

O leitor inicia consultando as etiquetas com o comando Query $(Q)$ (informando o tamanho do quadro $2^{Q}$ ). Cada etiqueta seleciona um slot aleatório entre 1 e $2^{Q}$. O slot selecionado é codificado em Manchester e enviado ao leitor. Com esta informação o leitor identifica quais 
slots estão selecionados, permitindo assim a eliminação dos slots vazios. Então o leitor inicia a chamada de cada um dos slots, um por um. Se uma colisão é detectada, o leitor adiciona dois slots adicionais no final do quadro e as etiquetas em colisão do slot atual selecionam aleatoriamente um desses novos slots adicionais. O leitor continua chamando cada um dos slots restantes até o final do quadro (EOF - End Of Frame). Cada slot de colisão gera dois novos slots adicionais no final do quadro. Resultados de simulação mostraram que a proposta pode melhorar o desempenho em aproximadamente $25 \%$ quando comparado ao Algoritmo Q em relação a métrica total de slots.

\subsubsection{Wang et al. (2014)}

Wang et al. (2014) propuseram um novo algoritmo anticolisão baseado na proposta EDFSA (Lee et al., 2005), chamado de Group Improved Enhanced Dynamic Frame Slotted Aloha (GroupIEDFSA), ilustrado na Figura 3.15.

A proposta foi desenvolvida para aplicações onde a quantidade de etiquetas é previamente conhecida, como supermercados, linhas de produção entre outros. Neste algoritmo, quando o número de etiquetas é muito maior que o tamanho de máximo de quadro permitido, as etiquetas são divididas em grupos e apenas um único grupo é selecionado para responder por vez. Após a primeira rodada de identificação, o número esperado de etiquetas não identificadas é adicionado ao número de etiquetas de um grupo e comparado com 354. Se este número é menor que $354^{4}$, um novo grupo mesclado é criado; caso contrário o conjunto de etiquetas não identificadas será identificado separadamente.

O leitor inicia definindo o tamanho inicial do quadro e realizando a primeira rodada de identificação. Após a primeira rodada. é possível obter o valor esperado da quantidade de etiquetas não identificadas pela Equação 3.29, que por sua vez necessita dos resultados das Equações 3.27 e 3.28 (PASSO 1). O leitor compara então este valor com 354 para determinar se este conjunto precisa ser dividido em grupos. Se o número for maior do que 354, as etiquetas precisam ser reagrupadas (PASSO 2). Posteriormente o leitor envia o valor $m$, onde $m$ é o número de grupos para que as etiquetas escolham um valor aleatório $([0, m-1])$. O grupo 0 será o próximo a ser chamado. Os demais grupos entram em estado de espera.

\footnotetext{
${ }^{4}$ Esta quantidade foi selecionada para manter a eficiência do sistema acima de $34.6 \%$.
} 


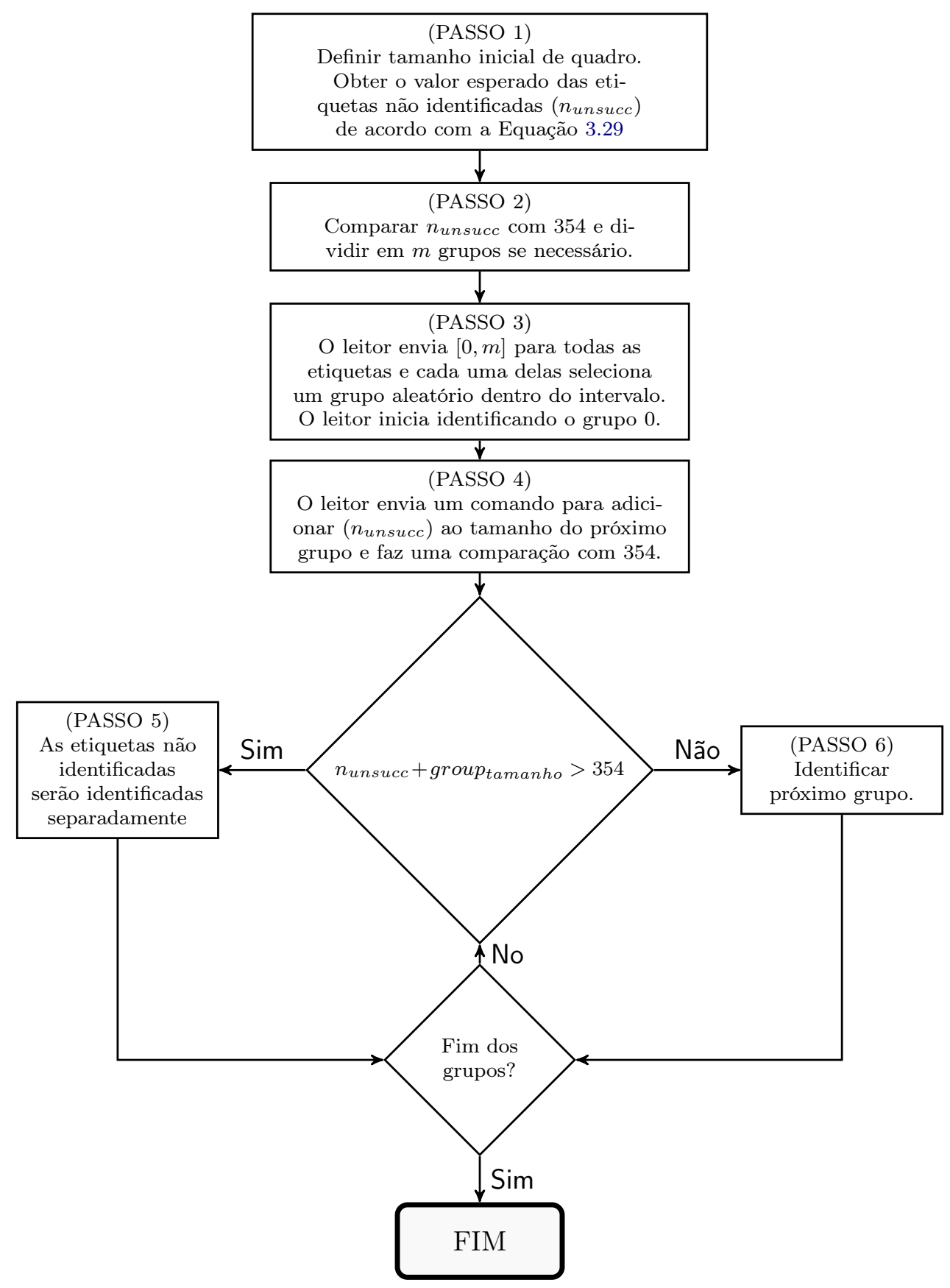

Figura 3.15: Fluxograma da proposta de Wang et al. (2014).

Então, ao final da rodada, é possível obter o valor esperado da quantidade de etiquetas não identificadas. O leitor envia um comando para que este número seja adicionado ao tamanho do próximo grupo e comparado com 354 (PASSO 4). Caso esta soma seja maior, as etiquetas não identificadas serão identificadas separadamente (PASSO 5). Caso contrário as etiquetas não identificadas serão mescladas com o próximo grupo e o leitor inicia a próxima rodada (PASSO 6). O processo termina quando todos os grupos são identificados. Resultados de simulação mostram que o GroupIEDFSA supera o desempenho com relação ao EDFSA em 
pelo menos $17 \%$ aproximadamente, quando levada em consideração a métrica tempo de identificação.

$$
\begin{gathered}
p_{\text {succ }}=\left(\begin{array}{c}
n \\
1
\end{array}\right) \times \frac{1}{f} \times\left(1-\frac{1}{f}\right)^{n-1} \\
n_{\text {succ }}=f \times p_{\text {succ }} \\
n_{\text {unsuc }}=n-n_{\text {succ }}
\end{gathered}
$$

\subsubsection{Xu e Chen (2015)}

Xu e Chen (2015) propuseram um melhoramento da proposta de He e Wang (2013) denominado Improved RFID Anti-collision Algorithm (IAA). O algoritmo inicialmente detecta a distribuição dos slots para saber quais foram selecionados, eliminando os vazios $\left(S_{0}\right)$. Posteriormente, o número de etiquetas é estimado ( $\tilde{n}$ - Equação 3.30) baseado na proposta de Khandelwal et al. (2006). Após estimar $\tilde{n}$, o leitor pode calcular a quantidade de slots de sucesso (Equação 3.31) e de colisão (Equação 3.32). Posteriormente, calcula-se a média de etiquetas em cada slot de colisão através da Equação 3.33. O arredondamento do logaritmo de $\tilde{x}$ é calculado conforme Equação 3.34. O valor de $q$ representa a quantidade de slots adicionais que serão adicionados ao final do quadro para identificação das etiquetas em colisão. Neste ponto o algoritmo difere da proposta de He e Wang (2013) e resultados de simulação mostram que o algoritmo de Xu e Chen (2015) pode alcançar ganhos de até 33\% em relação a He e Wang (2013).

$$
\begin{gathered}
\tilde{n}=\frac{\ln \left(\frac{S_{0}}{L}\right)}{\ln \left(1-\frac{1}{L}\right)} \\
S_{1}=\tilde{n}\left(1-\frac{1}{L}\right)^{\tilde{n}-1} \\
S_{k}=L-S_{0}-S_{1}
\end{gathered}
$$




$$
\begin{gathered}
\tilde{x}=\frac{\tilde{n}-S_{1}}{S_{k}} \\
q=\operatorname{ceil}\left(\log _{2} \tilde{x}\right)
\end{gathered}
$$

Apesar de atingir eficiência aproximada de 0.55, maior que o máximo teórico de 0.43 do BTSA, os autores partem do princípio de que é possível identificar a distribuição de escolha dos slots, o que em situações reais não é possível (Wu et al., 2013) (Finkenzeller et al., 2010).

\subsection{Propostas que lidam com efeito captura}

A proposta denominada de GBT (General Binary Tree) (Lai e Hsiao, 2010) é uma proposta baseada em árvore que lida com o efeito captura. Para evitar que etiquetas tornem-se ocultas, o leitor envia o identificador da etiqueta decodificada para todo o conjunto de etiquetas. Dessa forma as etiquetas não identificadas no slot onde o efeito captura ocorreu podem participar da próxima rodada de identificação. Apesar de identificar todas as etiquetas, este processo produz um número excessivo de colisões, prejudicando o desempenho do processo.

A mesma estratégia utilizada pelo GBT, é utilizada pelo Algoritmo Q, o que também resulta em colisões excessivas. Maguire e Pappu (2009) propuseram um algoritmo para otimizar o algoritmo Q. O mesmo considera a ocorrência do efeito captura e permite que as etiquetas ocultas selecionem um novo slot em rodadas subsequentes. Seu mecanismo de funcionamento baseia-se no cálculo de backlog considerando-se que a quantidade de etiquetas e a probabilidade de efeito captura são valores conhecidos, fato este que não corresponde a cenários reais.

Wu e Zeng (2015) estenderam a proposta descrita em (Wu et al., 2013) para o ambiente do efeito captura. Assim como as propostas anteriores, os mesmos consideram a probabilidade de efeito captura como constante em quaisquer situações, o que não corresponde à realidade (Bekkali et al., 2015; Sanchez e Smith, 1999; Zorzi, 1997). 


\subsection{Resumo}

A Tabela 3.1 resume características dos trabalhos relacionados descritos neste capítulo. A coluna Taxonomia classifica a proposta de acordo com a classificação apresentada no início deste capítulo. A coluna Thr apresenta a métrica eficiência do sistema, que será detalhada no próximo capítulo, pois trata-se da métrica mais universalmente adotada pela maioria dos autores da literatura. Considerou-se o máximo valor obtido desta métrica apresentada por cada proposta. Estes valores podem apresentar variações de acordo com a quantidade de etiquetas. Já a coluna Complexidade considera a seguinte classificação: Alta quando o algoritmo usa recursão, logaritmos ou combinações; Média quando o algoritmo inclui funções interativas numéricas sem as operações consideradas como alta; Baixa se o algoritmo necessita apenas de operações fundamentais. A coluna Estável analisa se o mecanismo é sensível a quantidade de etiquetas, ou seja, se a métrica eficiência do sistema converge para um valor constante. Finalmente a coluna Energia/ Tempo sinaliza se a proposta analisa estes aspectos de alguma forma no artigo.

Analisando a Tabela 3.1 pode-se perceber que a eficiência do sistema (Thr) varia de 0.32 , para o caso da proposta de Schoute (1983) até 0.6 para a proposta de Xu e Chen (2015). Observa-se também que a maioria dos mecanismos mais eficientes possuem complexidade de média para alta, sem também levar em consideração aspectos importantes de tempo e energia. Outro aspecto importante que pode ser observado é a ausência de mecanismos que melhor dimensionem o tamanho inicial de quadro, com exceção a proposta de (Cui e Zhao, 2009). Dessa forma, esta tese visou suprir estas lacunas observadas na literatura a fim de se propor novos mecanismos de acesso ao meio para sistemas RFID, conforme será mostrado no Capítulo 5. A categoria híbrida pela possibilidade de unir características de outras categorias foi a escolhida nas propostas desta tese. 
Tabela 3.1: Tabela comparativa entre várias propostas da literatura.

\begin{tabular}{|c|c|c|c|c|c|}
\hline Proposta & Taxonomia & Thr & Complexidade & Estável & $\begin{array}{l}\text { Energia/ } \\
\text { Tempo }\end{array}$ \\
\hline Schoute (1983) & PDFSA-b & 0,32 & Baixa & Não & Não \\
\hline EPCglobal, GS1 Inc. (2015) & DFSA-2 & 0,34 & Baixa & Sim & Não \\
\hline Cui e Zhao (2009) & DFSA-2 & 0,41 & Média & Sim & Não \\
\hline Wang et al. (2009) & DFSA-2 & 0,36 & Média & Não & Não \\
\hline Cui e Zhao (2010) & PDFSA-b & - & Média & Não & Sim \\
\hline Eom e Lee (2010) & PDFSA-b & 0,34 & Alta & Não & Não \\
\hline Zhu e Yum (2010) & PDFSA-b & 0,36 & Alta & Não & Não \\
\hline Wu e Zeng (2010) & PDFSA-b & - & Alta & Não & Sim \\
\hline Tong et al. (2012) & PDFSA-b & 0,355 & Alta & Não & Não \\
\hline He e Wang (2013) & HYB & 0.45 & Média & Não & Não \\
\hline Wu et al. (2013) & BTSA & 0,41 & Média & Sim & Não \\
\hline Mota e Batista (2014) & HYB & 0,39 & Baixa & Sim & Não \\
\hline Wang et al. (2014) & HYB & - & Média & Não & Sim \\
\hline Yao et al. (2014) & PDFSA-b & $\cong 0.418$ & Alta & Não & Não \\
\hline Shakiba et al. (2014) & PDFSA-b & 0,40 & Média & Não & Sim \\
\hline Chen (2014) & DFSA-2 & 0,35 & Baixa & Não & Não \\
\hline Xu e Chen (2015) & HYB & 0,6 & Alta & Não & Não \\
\hline Chen (2015) & DFSA-2 & - & Média & Sim & Sim \\
\hline Lin et al. (2015) & PDFSA-b & 0,42 & Alta & Não & Sim \\
\hline
\end{tabular}




\section{Capítulo 4}

\section{Métricas de Avaliação de Desempenho}

Neste capítulo, as principais métricas de análise de desempenho de algoritmos anticolisão adotadas na literatura são revisadas e categorizadas. A medição de desempenho de algoritmos anticolisão, como tempo de identificação, consumo de energia, perda de pacotes entre outros é uma tarefa desafiadora, devido à heterogeneidade de medidas adotadas por diferentes autores, além da diversidade de características e requisitos dos mais diversos cenários de aplicações. Dessa forma, a ausência de padronização na literatura sobre como avaliar o desempenho dos protocolos/algoritmos anticolisão propostos, torna de grande relevância o estudo apurado e abrangente sobre o assunto.

A análise de desempenho de protocolos anticolisão para sistemas RFID baseia-se em cinco categorias principais, conforme ilustrado na Figura 4.1: slots, tempo, energia, tráfego e precisão. A categoria slots está detalhada na Seção 4.1 e representa o tipo mais utilizado na literatura, pois baseia-se na contagem dos slots $^{1}$ utilizados no conjunto de quadros gerados no processo de identificação. Já a categoria tempo, descrita na Seção 4.2, visa medir os diversos tempos gastos no processo de identificação e é dependente dos hardwares envolvidos. O consumo de energia, abordado na Seção 4.3, está diretamente ligado ao tempo, porém com particularidades próprias, também dependentes dos hardwares envolvidos. O tráfego é analisado na Seção 4.4 e tem como objetivo contabilizar a troca de mensagens entre leitor e etiquetas, também durante a identificação. Finalmente, a precisão, na Seção 4.5, visa medir quão precisos são os algoritmos em relação às estimativas que os mesmos fazem em relação

\footnotetext{
${ }^{1}$ Desperd: Slots desperdiçados.
} 
ao tamanho da população de etiquetas.

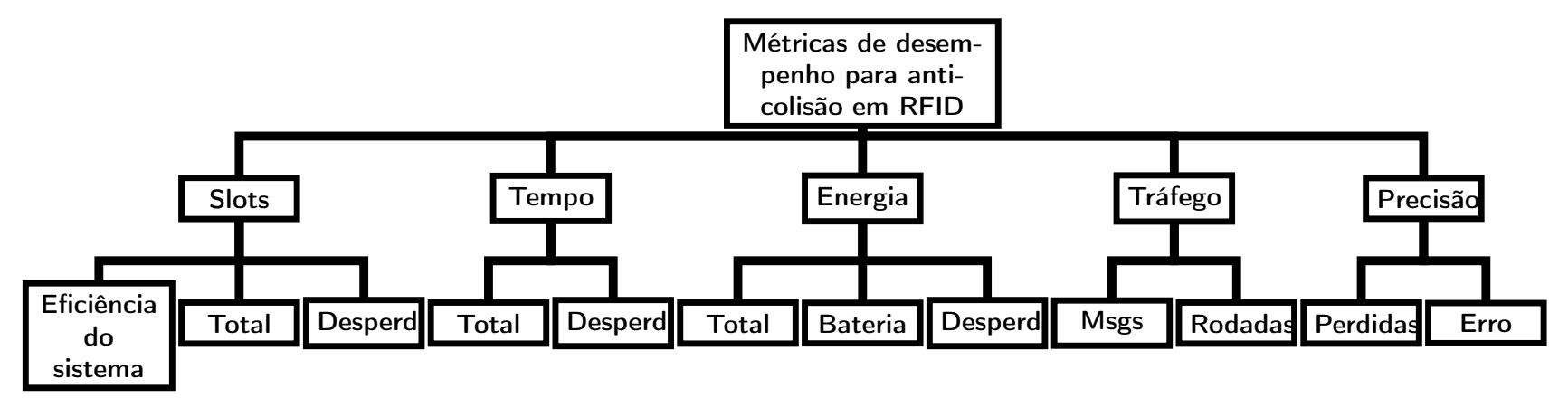

Figura 4.1: Taxonomia de métricas de avaliação de desempenho.

\subsection{Slots}

A categoria slots é a mais amplamente utilizada na avaliação de desempenho de sistemas RFID pois universalizam as medidas de desempenho dos mecanismos anticolisão, independente da implementação, facilitando as comparações entre as propostas. Apesar disso as métricas das demais categorias podem tornar-se importantes a depender dos objetivos da aplicação. Além disso, muitas propostas podem obter desempenhos semelhantes neste tipo de métrica, porém vantagens em outro tipo.

Com o objetivo de melhor entendimento desta categoria, precisa-se relembrar como funciona o FSA. Em resumo, o leitor gera um conjunto de quadros até que todas as etiquetas sejam identificadas (ou nenhuma colisão seja detectada no último quadro). Cada quadro consiste de um número específico de slots. Cada etiqueta não identificada escolhe no final de cada quadro, um slot aleatório para responder. Quando o leitor consulta um slot pode-se observar três tipos de resposta: sucesso, se apenas uma etiqueta respondeu; colisão, se mais de uma etiqueta respondeu; vazio, se nenhuma resposta foi detectada. Colisões e slots vazios significam que nenhuma etiqueta foi identificada, consistindo em desperdício de recurso. Dessa forma, se estes tipos de slots aumentam, o desempenho geral diminui. Com o número total de slots utilizados, pode-se definir as principais métricas baseadas em slots, conforme mostrado nas Equações 4.1, 4.2 e 4.3.

$$
\text { Total }_{\text {slots }}=\sum c o l+s u c+i d l
$$


A Equação 4.1 representa o somatório de todos os tipos de slots utilizados. Esta métrica isoladamente não é fácil de ser interpretada, mas é a métrica base para obtenção de outras métricas de maior facilidade de entendimento, como por exemplo a eficiência do sistema, mostrada na Equação 4.2. A métrica $S_{e f}$ é a mais utilizada para avaliar o desempenho de protocolos anticolisão em sistemas RFID, pois pode ser facilmente interpretada como um valor entre 0 e 1 ou um percentual. Apesar de ser a métrica mais universalmente utilizada, em muitos casos não é a mais adequada, pois não leva em consideração aspectos importantes da atualidade como tempo e energia consumida.

$$
S_{\text {ef }}=\frac{\text { suc }}{\text { Total }_{\text {slots }}}
$$

Como a interpretação de percentuais é rápida e fácil na maioria das aplicações, propõe-se uma nova métrica denominada $G_{q}$, conforme mostra a Equação 4.3 (ganho em relação ao Algoritmo Q (EPCglobal, GS1 Inc., 2015)) para medir o percentual de ganhos em desempenho em relação ao padrão C1G2 definido em EPCglobal, GS1 Inc. (2015). Dessa forma pode-se rapidamente medir quão melhor é um determinado algoritmo em comparação com o atual padrão utilizado na prática.

$$
G_{q}=\left|\frac{S_{e f}^{q}-S_{e f}^{X}}{S_{e f}^{q}}\right|
$$

A partir do exemplo A.0.1 pode-se observar que a utilização do algoritmo de Mota e Batista (2014) para identificar um conjunto de 3100 etiquetas, produziu uma eficiência do sistema de $\cong 41.2 \%$, que representa um ganho em relação ao algoritmo $\mathrm{Q}$ de $\cong 21.2 \%$.

\subsection{Tempo de identificação}

O tempo de identificação é uma das métricas mais facilmente interpretadas por usuários e desenvolvedores, uma vez que é uma métrica utilizada em vários aspectos do dia a dia. A contagem de slots não leva em consideração os diferentes custos de cada tipo. Por exemplo, a duração de tempo de um slot é usualmente utilizada em simulações como 1 ms (milissegundo) (considerando uma velocidade de $100 \mathrm{Kbps}$ e identificador de 96 bits). Além disso, os 
tempos dos slots variam dependendo se são de sucesso, colisão ou vazios (Namboodiri et al., 2012). A Tabela 4.1 mostra estas três diferentes classificações quanto ao tempo dos slots. A segunda coluna representa a medida de tempo observada em leitores baseados no protocolo proprietário I-CODE ${ }^{2}$ (Li et al., 2010). As colunas três e quatro possuem valores baseados no padrão EPCGlobal C1G2 para sistemas UHF passivos (EPCglobal, GS1 Inc., 2015). A coluna dois é baseada em valores práticos quando utilizado um leitor do tipo SkyeTek SkyeModule M9 Compact Flash RFID operando em 860-960 MHz com velocidade de $40 \mathrm{kbps}$ (Namboodiri et al., 2012). Finalmente a coluna três mostra as equações teóricas, descritas em EPCglobal, GS1 Inc. (2015), utilizadas para calcular os tempos dos slots (Chen, 2015).

Tabela 4.1: Tempo de identificação (em ms) para diferentes tipos de slots.

\begin{tabular}{|c|c|c|c|}
\hline Tipo de Slot & Li et al. (2010) & Namboodiri et al. (2012) & Chen (2015) \\
\hline Sucesso $\left(t_{s u c}\right)$ & 0.8 & 8 & $2 \times\left(T_{1}+T_{2}\right)+T_{R N 16}+T_{E P C+P C+C R C 16}=1375 \mu \mathrm{s}$ \\
\hline Colisão $\left(t_{c o l}\right)$ & 0.8 & 1.9 & $\left(T_{1}+T_{2}\right)+T_{R N 16}=337.5 \mu \mathrm{s}$ \\
\hline Vazio $\left(t_{i d l}\right)$ & 0.4 & 0.61 & $\left(T_{1}+T_{3}\right)=67.5 \mu \mathrm{s}$ \\
\hline
\end{tabular}

Segundo Namboodiri et al. (2012), as proporções entre os tempos de outros leitores em conformidade com o padrão EPC C1G2, são equivalentes. Dessa forma chegamos a uma proporção média de tempo entre os slots de colisão e vazios conforme as Equações 4.4, 4.5 e 4.6 para sistemas HF(Li et al., 2010), UHF-1(Namboodiri et al., 2012) e UHF-2(Chen , 2015) respectivamente.

$$
\begin{aligned}
& t_{i d l}=50 \% t_{c o l} \\
& t_{i d l}=32 \% t_{c o l} \\
& t_{i d l}=20 \% t_{c o l}
\end{aligned}
$$

Os parâmetros usuais utilizados por leitores baseados no C1G2 são apresentados na Tabela 4.2 (Chen, 2015; EPCglobal, GS1 Inc., 2015). Utilizando estes valores é possível calcular os tempos teóricos de cada tipo de slot de acordo com a coluna 3 da Tabela 4.1.

Sejam $t_{c o l}, t_{s u c}, t_{i d l}$ os tempos de duração de um slot de colisão, sucesso e vazio respecti-

\footnotetext{
${ }^{2}$ http://www.nxp.com/documents/data_sheet/SL092030.pdf
} 
Tabela 4.2: Parâmetros típicos do protocolo C1G2

\begin{tabular}{|c|c|}
\hline Parâmetro & Valor \\
\hline Taxa de transmissão & 80kbps \\
\hline $\begin{array}{l}R T_{\text {cal }} \text {, ou tempo de calibração do leitor- } \\
\text { etiqueta }\end{array}$ & $31.25 \mu \mathrm{s}$ \\
\hline TRate, ou taxa de transmissão das etiquetas & $125,80,64.5,31.25,15.625 \mathrm{Kbps}$ \\
\hline$L F$ ou frequência de sinal de sub-portadora & $160 K h z$ \\
\hline$T_{p r i}$ ou intervalo de repetição de pulso & $\frac{1}{L F}$ \\
\hline $\begin{array}{l}T_{1} \text { ou tempo entre a solicitação do leitor e uma } \\
\text { imediata resposta de etiqueta }\end{array}$ & $\operatorname{Max}\left\{R T_{\text {cal }}, 10 * T_{\text {pri }}\right\}=62.5 \mu \mathrm{s}$ \\
\hline$T_{2}$ ou tempo de resposta de uma etiqueta & $5 \times T_{p r i}=62.5 \mu s$ \\
\hline $\begin{array}{l}T_{3} \text { ou tempo de espera depois de } T_{1} \text { antes de } \\
\text { transmitir outro comando }\end{array}$ & $5 \times T_{p r i}=5 \mu s$ \\
\hline Tempo de uma Query $\left(T_{q}\right)$ & $412.5 \mu \mathrm{s}$ \\
\hline Tempo do RN16 $\left(T_{R N 16}\right)$ & $212.5 \mu \mathrm{s}$ \\
\hline Tempo do ACK $\left(T_{A C K}\right)$ & $337.5 \mu \mathrm{s}$ \\
\hline $\begin{array}{lcl}\text { Tempo } & \text { do } & \text { EPC } / I D / C R C 16 \\
\left(T_{E P C+P C+C R C 16}\right) & \end{array}$ & $912.5 \mu \mathrm{s}$ \\
\hline
\end{tabular}

vamente, chega-se ao tempo total gasto na identificação do conjunto de etiquetas $T_{\text {total }}$.

$$
T_{\text {total }}=t_{\text {suc }} \times n+t_{c o l} \times c o l+t_{i d l} \times i d l
$$

Alguns autores (Chen, 2015; Shakiba et al., 2014; Wu et al., 2010) propõem uma métrica que combinam a eficiência do sistema $\left(S_{e f}\right)$ com tempo de identificação, denominada de Channel Usage Efficiency, ou eficiência de uso do canal $\left(C_{e f}\right)$. A métrica além de utilizar a quantidade de cada tipo de slot utiliza os tempos de cada um. A Equação 4.8 define o cálculo da métrica, que representa a relação entre o tempo dos slots de identificação e o tempo total.

$$
C_{e f}=\frac{t_{s u c} \times s u c}{\left(t_{c o l} \times c o l+t_{i d l} \times i d l+t_{s u c} \times s u c\right)}
$$

Chen (2015) utilizou uma métrica denominada de eficiência em economia de tempo (time-saving efficiency) que visa comparar os ganhos percentuais entre dois algoritmos. A métrica é definida conforme a Equação 4.9. Os parâmetros $T_{1}$ e $T_{2}$ são os tempos requeridos pelos algoritmos comparados, ou seja, está-se calculando os ganhos percentuais do algoritmo 
2 em relação ao algoritmo 1.

$$
T S_{e f}=\frac{T_{1}-T_{2}}{T_{1}} \times 100 \%
$$

Finalmente, a métrica mais facilmente interpretada e usualmente utilizada pelos fabricantes, a taxa de leitura de etiquetas por segundo é apresentada pela Equação 4.10, calculada como a relação entre o tempo gasto para identificar o conjunto de $n$ etiquetas $\left(T_{\text {total }}\right)$ e a quantidade de etiquetas $(n)$.

$$
\text { Tags }_{\text {sec }}=\frac{T_{\text {total }}}{n}
$$

\section{$4.3 \quad$ Energia}

O hardware de um leitor RFID é basicamente formado por uma unidade microcontroladora embutida (embedded micro-controller unit - $M C U$ ) e um circuito de leitura. As MCUs podem processar instruções em altas velocidades e baixo consumo de energia, por exemplo, 1 nJ por instrução (Klair et al., 2010). Para o correto entendimento do consumo de energia de um leitor durante o processo de identificação de um conjunto de etiquetas, precisa-se entender como o consumo energético é medido em equipamentos elétricos em geral. O principal parâmetro para o cálculo do consumo de energia é o tempo gasto durante a operação, denotado por $T_{\text {total }}$ na Equação 4.7. No caso específico de leitores RFID, este é o tempo gasto para identificar o conjunto de etiquetas, de acordo com a Equação 4.7. O parâmetro adicional é a potência do equipamento $(P)$. Para o cálculo da potência precisa-se saber a tensão elétrica (Volts) e a corrente elétrica (Amperes) no modo leitura do equipamento, para que o parâmetro $P$ (Equação 4.11) seja adequadamente obtido. Com o tempo de operação $\left(T_{\text {total }}\right)$ e a potência do equipamento $(P)$ pode-se calcular a energia consumida $E_{\text {total }}$ (Watts) através da Equação 4.12. De forma similar pode-se calcular a energia desperdiçada mudando $T_{\text {total }}$ por $T_{\text {wasted }}$ conforme mostra a Equação 4.13. Em geral, o valor de $P$ pode ser encontrado na descrição dos equipamentos.

$$
P=V \times I
$$




$$
\begin{gathered}
E_{\text {total }}=P \times T_{\text {total }} \\
E_{\text {wasted }}=P \times T_{\text {wasted }}
\end{gathered}
$$

Outra métrica interessante que pode ser derivada da Equação 4.12 é o número de etiquetas que pode ser lida pelo leitor durante a duração da bateria $\left(N_{\text {protocol }}\right)$. Para isso precisase primeiramente calcular a energia armazenada da bateria $(B)$. A Equação 4.14 mostra o cálculo de $B$. Primeiramente precisa-se obter dois valores usualmente informados pelo fabricante: o valor da capacidade em mAh e a voltagem (em geral impressos na própria bateria). Aplica-se então a Equação 4.14. Após calcular B (Joules) pode-se calcular o valor da métrica $N_{\text {protocol }}$, onde $E_{\text {total }}$ é a energia consumida para identificar uma ou um conjunto de etiquetas. O Exemplo A.0.7 do Apêndice A ilustra a situação.

$$
\begin{gathered}
B=\left(\frac{(m A h \times V)}{1000}\right) \times 3600 \\
N_{\text {protocol }}=\frac{B}{E_{\text {total }}}
\end{gathered}
$$

A tarefa de quantificar o consumo de energia e a quantidade de máxima de leituras pode tornar-se de grande utilidade para desenvolvedores e implementadores de aplicações práticas, o que torna estas métricas relevantes no estudo e avaliação de algoritmos e mecanismos anticolisão para sistemas RFID.

\subsection{Tráfego}

Antes de descrever as equações que medem os valores relacionados ao tráfego, precisa-se primeiramente entender como funciona a troca de mensagens durante o processo de identificação das etiquetas. Os leitores RFID enviam comandos para as etiquetas que estão sob sua área de cobertura para obter seus identificadores. Em sistemas passivos, apenas os leitores iniciam a comunicação. O leitor pode comunicar-se com as etiquetas utilizando três 
operações básicas: Select, Inventory, e Access. Como esta tese é especificamente relacionada ao processo de identificação (anticolisão), então o alvo serão os comandos relacionados ao processo que fazem parte da operação inventory.

O leitor inicia a fase de inventory transmitindo um comando Query para as etiquetas, usualmente informando o tamanho do quadro e outras informações específicas de cada protocolo. O leitor então recebe uma resposta denominada $R N 16$. Se esta resposta for recebida corretamente o leitor então devolve uma confirmação (ACK) após receber o ID da etiqueta. Em caso de um RN16 corrompido o leitor detecta uma colisão. Caso nenhum RN16 seja detectado então tem-se um slot vazio. A Figura 4.2 mostra resumidamente o fluxo de comandos trocados entre leitores e etiquetas em três possíveis situações: sucesso, colisão e vazio. Os comandos pontilhados são utilizados quando o protocolo é baseado em DFSA-2 enquanto os tracejados baseiam-se em PDFSA-b ou BTSA (ver Capítulo 3).

Com a informação de como os comandos são trocados, torna-se possível estimar o tráfego gerado (em bytes) durante o processo de identificação das etiquetas. Mesmo que o protocolo utilize outros comandos, pode-se facilmente obter novas equações para estimar o tráfego gerado. Baseado no número de slots, a Equação 4.16 é uma fórmula generalizada, a Equação 4.17 aplica-se para os tipos de protocolos baseados em PDFSA-b e BTSA e a Equação 4.18 é aplicada a protocolos DFSA-2. Os valores apresentados nas equações a seguir são baseados nas definições apresentadas em EPCglobal, GS1 Inc. (2015).

$$
\begin{aligned}
\text { Traffic } c_{\text {bytes }} \cong & \frac{\left(\text { suc } \times S p_{\text {size }}^{\text {suc }}\right)+\left(\text { col } \times S p_{\text {size }}^{\text {col }}\right)+\left(i d l \times S p_{\text {size }}^{\text {suc }}\right)}{8} \\
& \cong \frac{156 \times s u c+38 \times \mathrm{col}+22 \times i d l}{8} \\
& \cong \frac{160 \times s u c+47 \times \mathrm{col}+31 \times i d l}{8}
\end{aligned}
$$

Outra métrica que pode ser útil em várias situações é o número de rodadas geradas, em outras palavras, a contagem do número de quadros utilizados (frames). 
SLOT DE SUCESSO

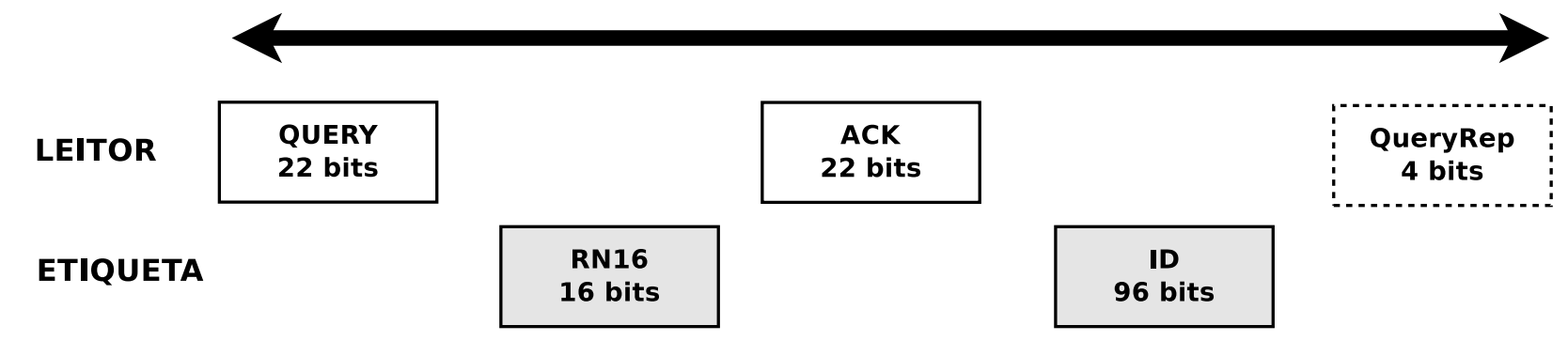

SLOT DE COLISÃO
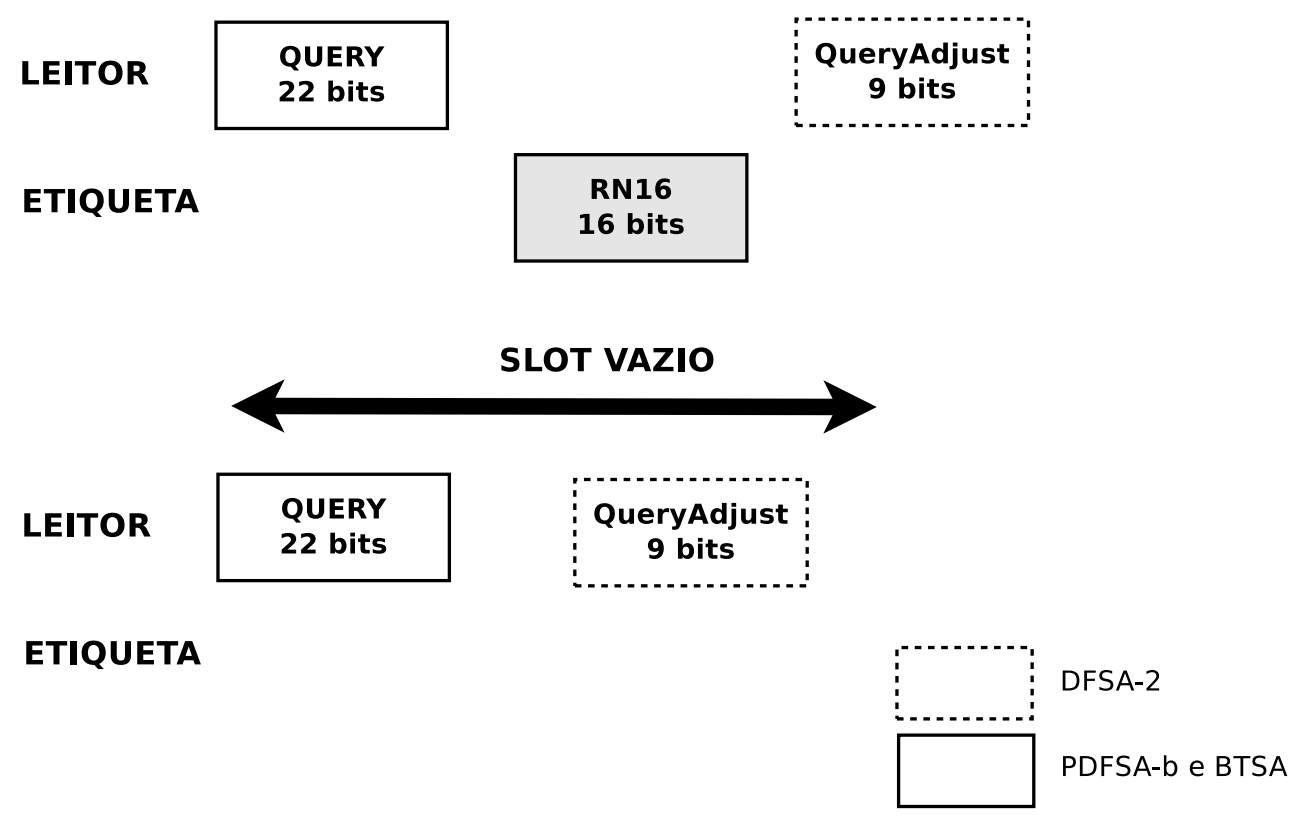

Figura 4.2: Tráfego entre leitor e etiquetas de acordo com o tipo de slot.

\subsection{Precisão}

A primeira métrica de precisão relacionada ao processo de identificação de etiquetas é relacionada com a taxa de identificação. Em outras palavras, é uma métrica que visa saber quantas etiquetas foram devidamente identificadas dentro do conjunto. Para ser utilizado na prática, um algoritmo anticolisão deve possuir este valor igual a 100\%. Caso contrário, pode-se ter a situação em que um leitor de um supermercado não identifica todos os itens do carro de compras, gerando prejuízos ao estabelecimento, por exemplo (Wu e Zeng, 2015). As Equações 4.19 (taxa de sucesso) e 4.20 (taxa de insucesso) mostram como calcular estes valores.

$$
R_{s}=\frac{s u c}{n} \times 100 \%
$$




$$
R_{m}=\frac{n_{\text {missed }}}{n} \times 100 \%
$$

A segunda métrica de precisão proposta visa avaliar quão precisos são os mecanismos de estimação do número de etiquetas para a definição dos tamanhos dos quadros. Neste caso, uma taxa de $100 \%$ não faz-se necessária pois é um parâmetro usado apenas para cálculo dos tamanhos dos quadros. O cálculo é similar ao $R_{s}$. A Equação 4.21 apresenta esta métrica como a relação entre o número de etiquetas estimadas $\left(n^{\prime}\right)$ e a quantidade real de etiquetas $(n)$. Então o resultado é multiplicado por 100 para obter-se o percentual. Quanto mais preciso for o mecanismo, maior serão várias das métricas de desempenho. No entanto a complexidade para chegar-se a valores mais precisos podem diminuir tal aumento de eficiência (Chen, 2014).

$$
E s t_{a c c}=\frac{n^{\prime}}{n} \times 100
$$

\subsection{Resumo}

A Tabela 4.3 resume as principais métricas adotadas na literatura. As métricas mais usualmente utilizadas na literatura concentram-se nas que lidam de alguma forma com a quantidade de slots utilizados como as métricas das linhas 1,2 e 3. Apesar de serem as mais utilizadas, estas métricas não levam em consideração os tempos de cada tipo de slot, tornando importantes as métricas das linhas 5,6 e 7, pois as mesmas consideram as diferenças entre cada tipo de slot. Apesar de importantes, muitas vezes as métricas que necessitam dos tempos (em milésimos de segundos) dos slots não são fáceis de serem obtidas, devido a grande variedade de tipos de leitores. Apesar desta dificuldade, ao londo do desenvolvimento desta tese, observou-se que em alguns casos, alguns algoritmos possuem desempenhos equivalentes na métrica eficiência do sistema, porém desempenhos diferentes quando observada alguma métrica relacionada ao tempo, como o consumo de energia, por exemplo. Dessa forma, os resultados obtidos no próximo capítulo levaram em consideração não apenas as métricas mais utilizadas que são as relacionadas à contagem dos slots, mas também as relacionadas com o tempo, como as mostradas nas linhas 6-11, permitindo dessa forma uma comparação 
mais justa, abrangente e aprofundada.

Tabela 4.3: Métricas de avaliação de desempenho.

\begin{tabular}{|c|c|c|c|}
\hline Linha & Métrica & Notação & Equação \\
\hline 1 & Eficiência do sistema & $S_{e f}$ & $\frac{n}{\overline{\text { Total } l_{\text {slots }}}}$ \\
\hline 2 & Total slots & Total $_{\text {slots }}$ & $\sum c o l+s u c+i d l$ \\
\hline 3 & Slots Desperdiçados & Total $_{\text {wasted }}$ & $\sum(c o l+i d l)$ \\
\hline 4 & Ganho comparado ao C1G2 & $G_{q}$ & $\frac{S_{e f}^{q}-S_{e f}^{X}}{S_{e f}^{q}}$ \\
\hline 5 & Latência & $T_{\text {total }}$ & $\left(t_{s u c} \times n\right)+\left(t_{c o l} \times c o l\right)+\left(t_{i d l} \times i d l\right)$ \\
\hline 6 & Tempo desperdiçado & $T_{\text {wasted }}$ & $\left(t_{c o l} \times c o l\right)+\left(t_{i d l} \times i d l\right)$ \\
\hline 7 & Eficiência de uso do canal & $C_{e f}$ & $\frac{t_{s u c} \times s u c}{\left(t_{c o l} \times c o l+t_{i d l} \times i d l+t_{s u c} \times s u c\right)}$ \\
\hline 8 & Eficiência de economia de tempo & $T S_{e f}$ & $\frac{T_{1}-T_{2}}{T_{1}} \times 100 \%$ \\
\hline 9 & Etiquetas por segundo & Tags $_{s e c}$ & $\frac{T_{\text {total }}}{n}$ \\
\hline 10 & Consumo de energia total & $E_{\text {total }}$ & $V \times I \times T_{\text {total }}$ \\
\hline 11 & Consumo de energia desperdiçado & $E_{\text {wasted }}$ & $V \times I \times T_{\text {wasted }}$ \\
\hline $\begin{array}{l}12 \\
13 \\
14\end{array}$ & $\begin{array}{l}\text { Número total de bytes transmitidos } \\
\text { Taxa de insucesso (perda) } \\
\text { Taxa de sucesso }\end{array}$ & $\begin{array}{c}\text { Trafficbytes } \\
R_{m} \\
R_{s}\end{array}$ & $\begin{array}{c}\left((s u c+c o l) \times P_{\text {size }}^{t}\right)+\left(S_{\text {total }} \times P_{\text {size }}^{r}\right) \\
\frac{n_{\text {missed }}}{n} \times 100 \% \\
\frac{s u c}{n} \times 100 \%\end{array}$ \\
\hline 15 & Precisão de estimação & Estacc & $\frac{n^{\prime}}{n} \times 100$ \\
\hline
\end{tabular}




\section{Capítulo 5}

\section{Mecanismos propostos}

Este capítulo apresenta os resultados obtidos durante o desenvolvimento desta tese. Cada uma das subseções apresenta um resultado explicando a metodologia e resultados obtidos.

Um aspecto chave observado no Capítulo 3 é a ausência de mecanismos eficientes em pontos como o consumo de energia e tempo de identificação. A maioria das propostas da literatura não levam em consideração também a importância da correta definição do tamanho de quadro inicial. Dessa forma, para suprir estas ausências, são propostos dois algoritmos que diminuem o consumo de energia e o tempo em relação ao padrão C1G2: NEDFSA e IDFSA-IcolR, um mecanismo que diminui a perda de pacotes em cenários com etiquetas em movimento (Welbourne et al., 2009) e um algoritmo anticolisão para funcionamento em ambientes sob o efeito captura, IDFSA-IcolR-EC. Além disso são propostas duas aplicações para a avaliação de desempenho em sistemas RFID: nsRFIDsim, um módulo para o simulador de eventos discretos ns-2 e o jRFIDsim, uma suíte de benchmark para avaliação e comparação de novos algoritmos com o estado da arte.

A organização das seções segue a ordem cronológica com que os softwares e mecanismos foram desenvolvidos. A Seção 5.1 descreve um mecanismo redutor de perda de pacotes em cenários com mobilidade. A Seção 5.2 apresenta o nsRFIDsim. Já a Seção 5.3 apresenta o NEDFSA, seguido pelo jRFIDsim na Seção 5.4 e pelos algoritmos IDFSA-IcolR e IDFSAIcolR-EC nas Seções 5.5 e 5.6 respectivamente. 


\subsection{Um Mecanismo para redução de respostas repetitivas em RFID}

O objetivo desta seção é apresentar um mecanismo de QoS para cenários com mobilidade dos objetos cujos nós sejam etiquetas RFID e que sejam sensíveis a perda de pacotes. O mecanismo proposto teve seu desempenho avaliado por meio de experimentos simulados no simulador ns-2, modificado com a inclusão do módulo apresentado na Seção 5.2, e os resultados confirmam a eficácia do mesmo. Por exemplo, em um cenário, a perda de pacotes que era de $10 \%$ a $42 \%$ foi reduzida para menos de $3 \%$ quando o mecanismo foi utilizado. $\mathrm{O}$ mecanismo foi também avaliado quando utilizado o Algoritmo Q e o BTSA, apresentando ganhos médios no intervalo [6\%,37\%], com relação a quantidade reduzida de tráfego.

O conteúdo a ser apresentado a seguir com a proposta do mecanismo e a sua análise de desempenho difere dos trabalhos relacionados encontrados na literatura porque os experimentos simulam cenários RFID reais, com o ns-2 e quantidade de etiquetas variáveis, o que possibilita uma análise mais aprofundada do mecanismo de QoS proposto.

\subsubsection{Mecanismo proposto}

Conforme Welbourne et al. (2009), o excesso de pacotes trocados entre leitores e etiquetas RFID afeta significativamente a escalabilidade da rede e também as garantias de requisitos de QoS das aplicações. O mecanismo proposto visa reduzir essa quantidade de pacotes e pode ser aplicado a quaisquer mecanismos anticolisão propostos da literatura.

O mecanismo proposto tem como objetivo garantir a qualidade dos serviços oferecidos em cenários de localização e rastreamento, reduzindo as taxas de perdas de pacotes. Uma característica comum pode ser observada nestas situações: as etiquetas não precisam responder a todas requisições do leitor. O fato é justificado pois as etiquetas apenas precisam responder requisições vindas de leitores diferentes da requisição anterior, ou seja, o nó não precisa informar a localização, se a mesma não foi alterada. O mecanismo proposto está detalhado no Algoritmo 6. A Figura 5.1 ilustra a máquina de estados das etiquetas.

Observa-se que a etiqueta possui três estados: (i) "Esperando": a etiqueta está esperando uma requisição ou recebeu uma requisição do mesmo leitor que possui armazenado em sua 


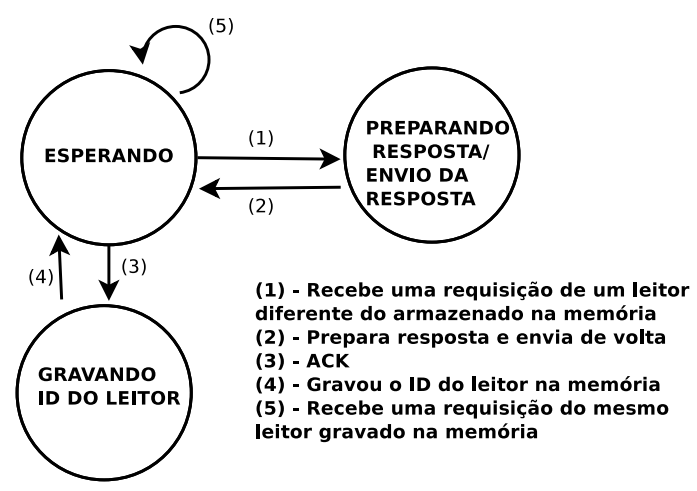

Figura 5.1: Máquina de estados das etiquetas para o mecanismo proposto

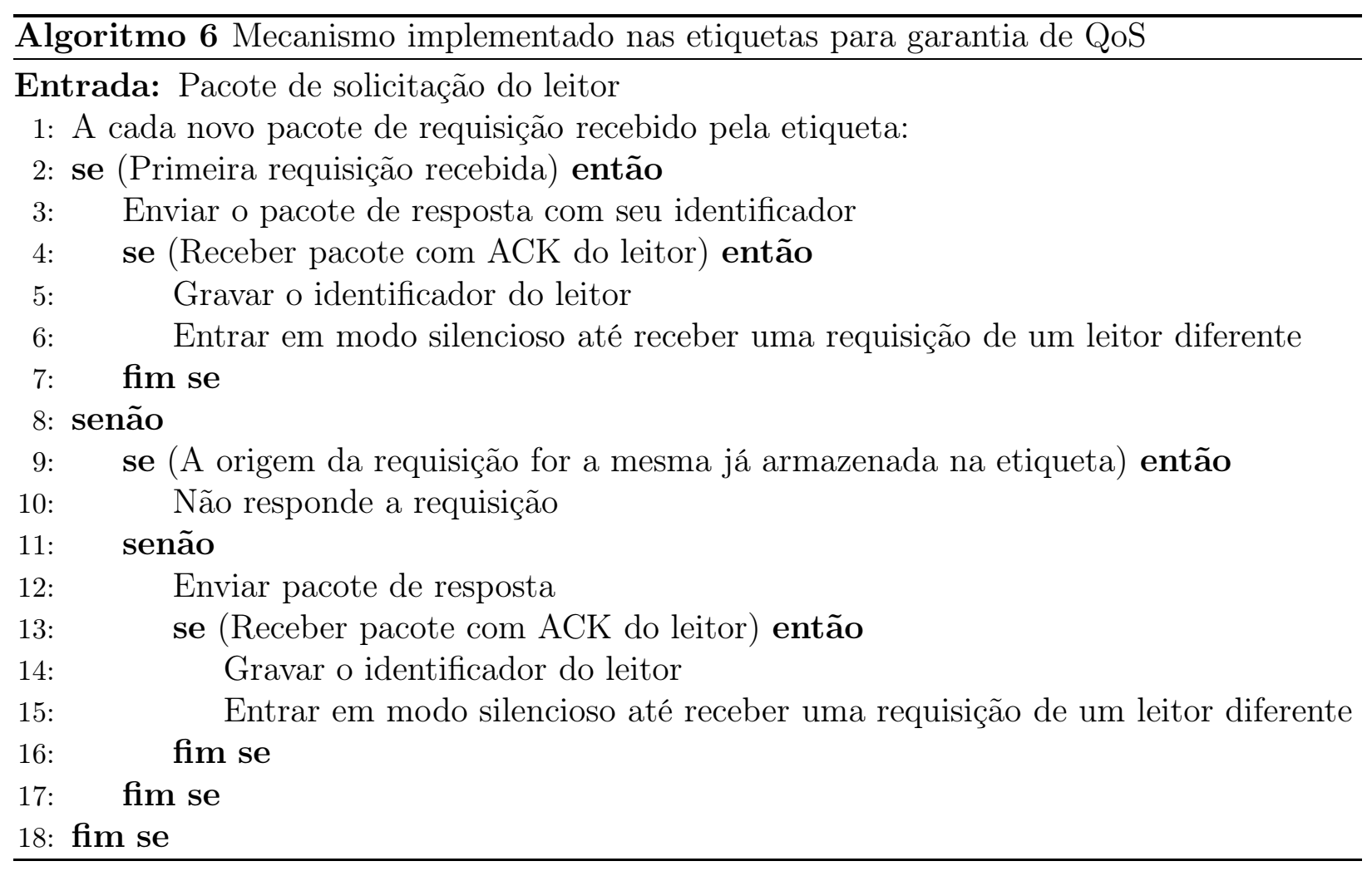

memória. Caso a etiqueta receba uma requisição de um leitor diferente a mesma passa para o estado (ii)"preparação e envio de resposta", e volta ao estado "esperando". Caso o leitor receba a resposta corretamente, o mesmo envia um ACK de confirmação para etiqueta, que no estado "esperando" passa para o estado (iii) "gravando ID do leitor" onde é armazenado o ID do novo leitor, e posteriormente volta-se ao estado "esperando". O Algoritmo 6, para implementação nas etiquetas realiza exatamente os passos descritos pelo diagrama de estados da Figura 5.1, ou seja, a etiqueta verifica a origem da requisição (linhas 2 e 9) e responde apenas se a origem for diferente do ID já armazenado na memória (linhas 3 e 12). Ao responder, a etiqueta grava a nova ID do leitor apenas ao receber um ACK de confirmação (linhas 13 a 15). 


\subsubsection{Cenários e experimentos}

A fim de avaliar o desempenho do mecanismo proposto na Seção 5.1.1, dois cenários reais com dispositivos RFID foram simulados. A lista abaixo descreve os dois cenários.

- Um cenário que simula uma sala de aula, com turmas de 30 a 430 alunos variando de 50 em 50 onde os alunos permanecem na sala durante a primeira aula (cinquenta minutos); saem para o intervalo (20 minutos); voltam para a segunda aula (cinquenta minutos); e saem do prédio onde se localiza a sala de aula. Foram utilizados três leitores, um na sala de aula, outro no pátio do intervalo e o último na saída do prédio. O cenário está ilustrado na Figura 5.2a;

- Um cenário com cinco leitores, representando parte de uma feira de exposições, e quantidade de nós entre 50 e 1050, variando de 100 em 100, com posições e movimentos aleatórios. Os nós representam tanto expositores quando visitantes da feira. Há diversos leitores distribuídos pela feira e os nós estão frequentemente se movimentando pelas áreas de cobertura dos diversos leitores. A Figura 5.2b ilustra o cenário.

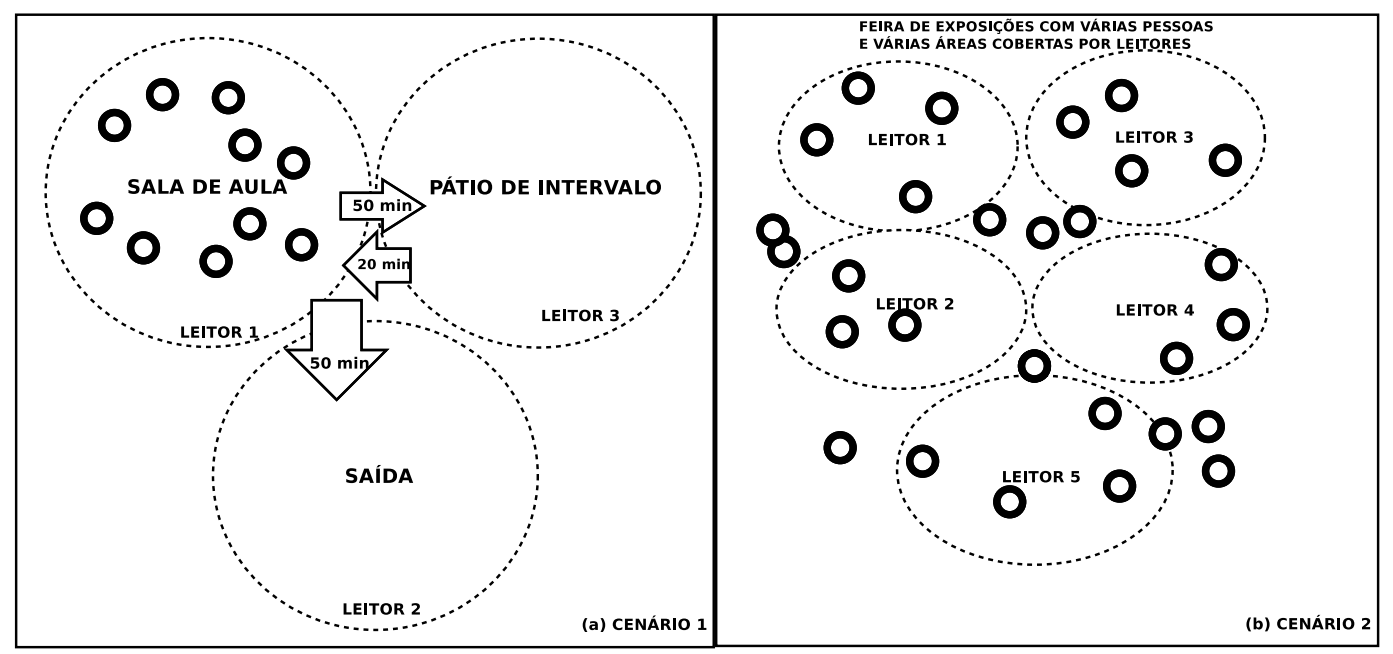

Figura 5.2: Cenários modelados

Os seguintes parâmetros foram levados em consideração:

- O tempo do experimento para o cenário (i) foi de cento e dez minutos, com leituras enviadas a cada três segundos. Para o cenário (ii) simulou-se trinta minutos, com solicitações a cada cinco segundos. 
- O mecanismo RTS/CTS - Request to Send / Clear to Send - (Requisição para enviar / Livre para enviar) e o protocolo de roteamento foram desabilitados por não fazerem parte do padrão de comunicação RFID (Finkenzeller et al., 2010). Estes parâmetros foram ajustados a partir do protocolo de acesso ao meio, simulando assim o MAC do sistema RFID.

- A potência de transmissão - Pt foi diminuída a fim de alcançar valores próximos dos reais, de um a dez metros, com os parâmetros Pt_0.28 $(\mathrm{mW})$ e RXThresh_ (potência minima para que os pacotes sejam recebidos pelos receptores) 2.12249e-07 (W).

- As taxas de transmissão dos canais leitor-etiqueta e etiqueta-leitor foram definidas como 9Kbps e 128Kbps (Finkenzeller et al., 2010), respeitando valores reais.

Cada cenário foi executado vinte vezes para cada quantidade de nós. O percentual de perda de pacotes para cada simulação foi calculado através da média aritmética entre as vinte medições, conforme a Equação 5.1. A Equação 5.2 mostra a fórmula de cálculo do tráfego gerado (quantidade de Kilobytes transferidos) pelas respostas das etiquetas, no sentido etiquetaleitor. As simulações foram realizadas em um servidor equipado com processador Intel Core i7-2700K 3,5GHz, 16GB de memória RAM e 1TB de espaço em disco rodando o sistema operacional Debian GNU/Linux versão 6.0.

$$
T p=\frac{\sum_{i=1}^{20}\left(\frac{\sum_{j=1}^{q l}\left(\frac{\sum_{k=1}^{s}\left(\frac{d_{k}}{d_{k}+r_{k}}\right)}{s}\right)}{q l}\right)}{20}
$$

$$
Q t=\frac{\sum_{i=1}^{20}\left(\frac{\left(d_{i}+r_{i}\right) * 8}{1000}\right)}{20}
$$


onde:

- $\mathrm{Tp}=$ Percentual de taxa de perda de pacotes $(0-1)$;

- Qt = Quantidade, em Kilobytes, de tráfego gerado pelas etiquetas aos leitores;

- $d_{i}=$ Quantidade de pacotes descartados na simulação $i$;

- $r_{i}=$ Quantidade de pacotes recebidos na simulação $i$;

- $\mathrm{i}=$ Número da simulação;

- $\mathrm{ql}=$ Quantidade de leitores;

- $\mathrm{s}=$ Quantidade de requisições;

- $d_{k}=$ Quantidade de pacotes descartados na requisição $k$;

- $r_{k}=$ Quantidade de pacotes recebidos na requisição $k$;

- 8 - Tamanho em bytes do pacote RFID.

\subsubsection{Resultados e discussão}

As Figuras 5.3 e 5.4 apresentam gráficos que mostram o percentual de perda de pacotes em função da quantidade de nós para os cenários modelados. São apresentadas as médias das medições e os intervalos de confiança com confiança de 95\%. Duas curvas são apresentadas em cada gráfico. Uma delas, identificada como "Cenário 1 sem mecanismo" apresenta os resultados sem a implementação do mecanismo proposto no Algoritmo 6. A outra curva, identificada como "Cenário 1 com mecanismo" apresenta os resultados com a implementação do mecanismo proposto no Algoritmo 6.

Observa-se no Cenário 1 (Figura 5.3) que o percentual de perdas de pacotes permanece constante quando a turma varia entre 30 e 180 alunos, mantendo uma taxa de perdas abaixo de 5\%. Este fato é justificado pela característica do leitor que consegue lidar bem com estas quantidades de nós. Percebe-se também que após 230 alunos, a taxa de perda, quando o mecanismo proposto não é usado, cresce significativamente, caracterizando que o leitor está no limite de sua capacidade de leituras, gerando perdas que podem inviabilizar a implantação 
do cenário. Ainda na Figura 5.3 pode-se observar que o mecanismo de QoS proposto levou os índices de perdas para um valor constante baixo, independente da quantidade de etiquetas, viabilizando a implementação do cenário sem que haja a necessidade de aquisição de novos leitores. Comparando o percentual de perdas no cenário com 430 nós observa-se que houve uma diminuição de aproximadamente $95 \%$ quando o mecanismo de QoS foi utilizado.

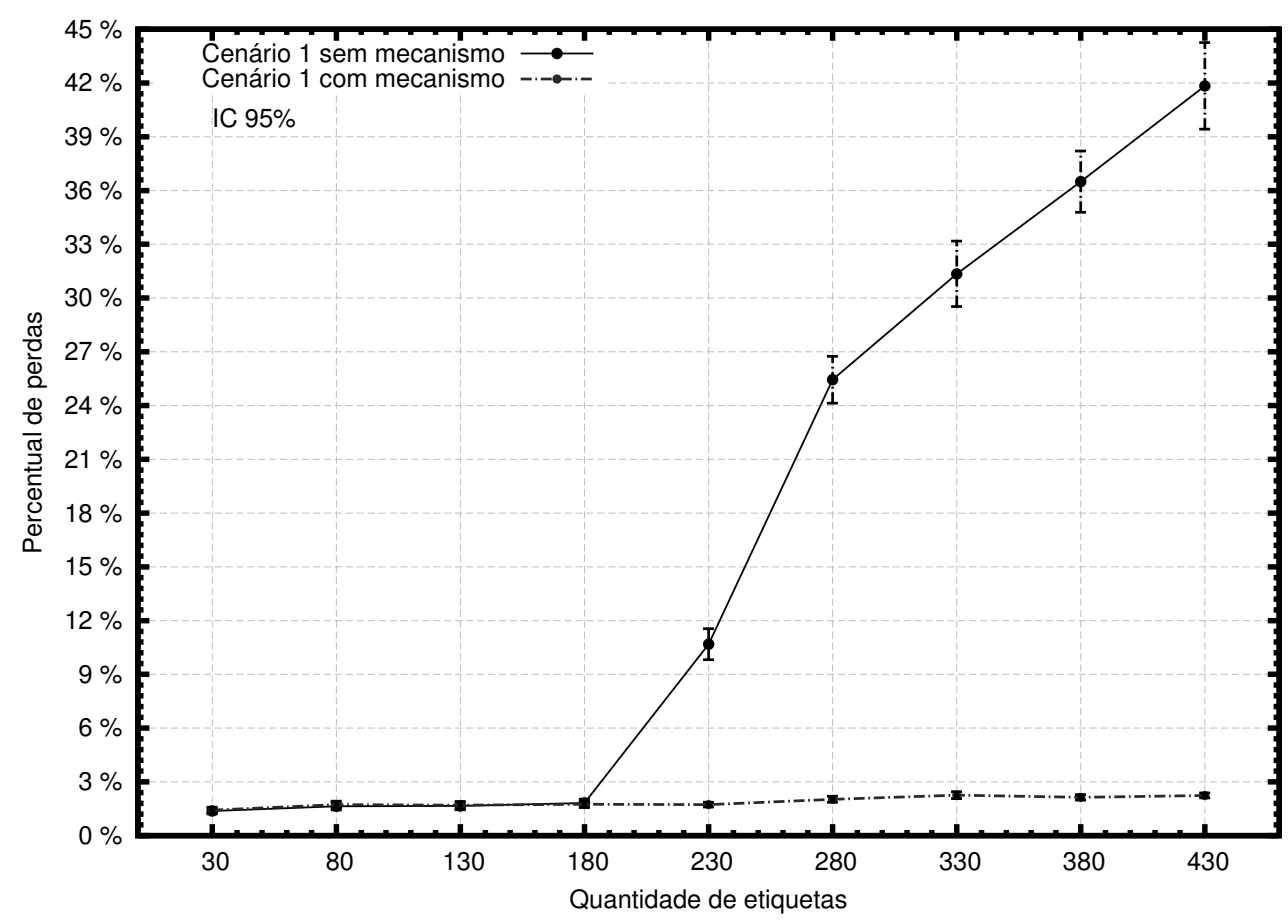

Figura 5.3: Percentual de perdas $x$ Quantidade de etiquetas para o Cenário 1

O Cenário 2, que simula uma feira de exposições, onde diversas pessoas movimentam-se aleatoriamente por vários locais diferentes, revela pequenos percentuais de perdas até os 450 nós, conforme Figura 5.4. Quando o mecanismo proposto não foi utilizado, as perdas chegaram a quase $18 \%$ quando haviam até 1050 etiquetas. O mecanismo proposto mostrouse bastante eficiente, pois assim como no Cenário 1, a curva permanece praticamente com valor constante pequeno. Comparando os resultados quando haviam 1050 etiquetas, observase que a utilização do mecanismo levou a uma redução de $81 \%$ na porcentagem de perdas de pacotes.

As Figuras 5.5 e 5.6 apresentam a quantidade de Kilobytes transferidos na rede nos Cenários 1 e 2 respectivamente. Como era de se esperar, a implementação do mecanismo de QoS reduz significativamente a quantidade de bytes na rede, o que trouxe como consequência a redução na taxa de perdas. Por exemplo, no Cenário 1, com 430 nós, a quantidade de bytes 


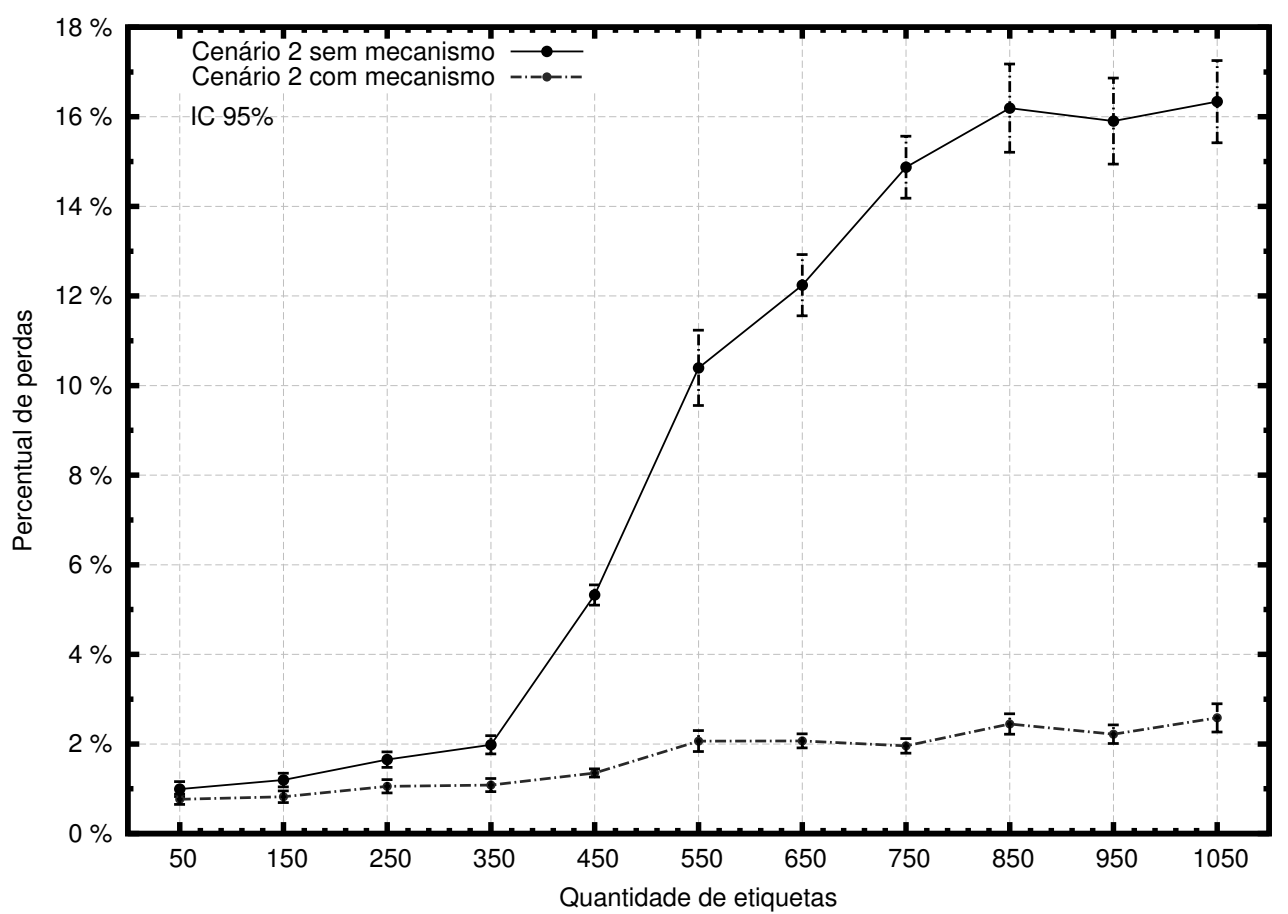

Figura 5.4: Percentual de perdas $x$ Quantidade de etiquetas para o Cenário 2

foi reduzida em 84\%. No cenário 2, a redução com a quantidade de 1050 etiquetas, foi de $63 \%$.

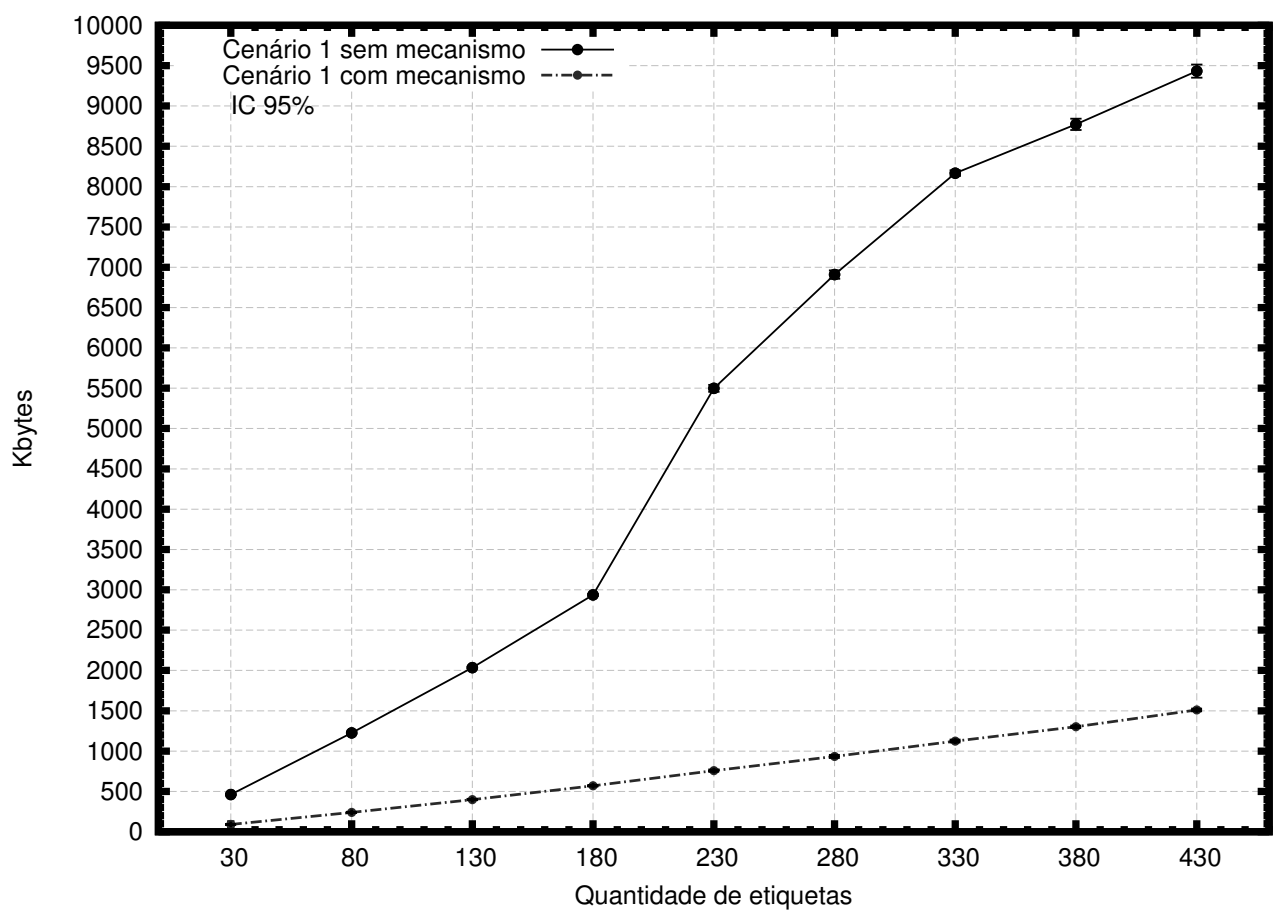

Figura 5.5: Quantidade média de Kilobytes transferidos no Cenário 1

O mecanismo proposto também foi avaliado em comparação com o Algoritmo Q e o BTSA, um algoritmo proposto em 2013 e comprovadamente eficiente em diferentes métricas. 


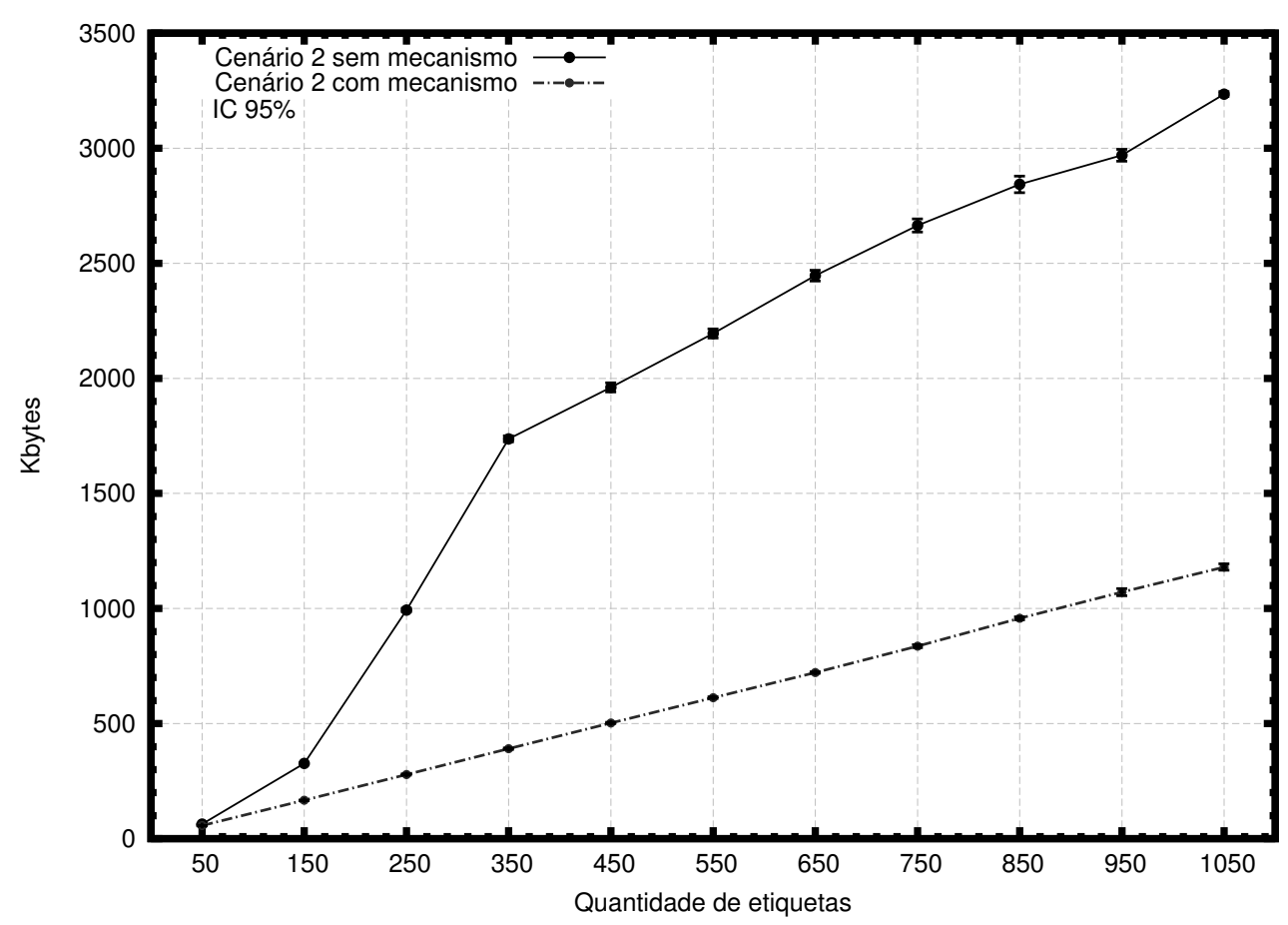

Figura 5.6: Quantidade média de Kilobytes transferidos no Cenário 2

Os resultados de simulação comprovaram que o mecanismo proposto é capaz de reduzir o número de pacotes gerados até mesmo quando comparado com o BTSA, conforme ilustra a curva de cor preta contínua da Figura 5.7. A Tabela 5.1 apresenta a diferença média máxima quando aplicados o Algoritmo BTSA e o mecanismo proposto, comparado ao Algoritmo Q. Por exemplo, quando tem-se 500 etiquetas, o BTSA reduz a quantidade de pacotes gerados em até $33 \%$, enquanto o mecanismo proposto reduz em até $44 \%$, ou seja, com diferença de $11 \%$ a favor do mecanismo proposto.

Tabela 5.1: Redução do número de pacotes gerados, comparados com o Algoritmo $Q$

\begin{tabular}{clll}
\hline Número de etiquetas & $\begin{array}{l}\text { Ganho médio } \\
\text { (BTSA) }\end{array}$ & $\begin{array}{l}\text { Ganho Médio } \\
\text { nismo Proposto) }\end{array}$ & $\begin{array}{c}\text { (Meca- Diferença } \\
\text { (BTSo }\end{array}$ \\
\hline $\mathbf{1 0 0}$ & $\cong 37 \%$ & $\cong 39 \%$ & $\cong 2 \%$ \\
$\mathbf{3 0 0}$ & $\cong 31 \%$ & $\cong 41 \%$ & $\cong 10 \%$ \\
$\mathbf{7 0 0}$ & $\cong 33 \%$ & $\cong 44 \%$ & $\cong 9 \%$ \\
$\mathbf{9 0 0}$ & $\cong 30 \%$ & $\cong 39 \%$ & $\cong 17 \%$ \\
\hline
\end{tabular}

\subsubsection{Conclusões parciais}

Esta seção propôs e avaliou o desempenho de um mecanismo de QoS para cenários sensíveis a perda de pacotes. Os resultados mostraram que o mecanismo implementado 


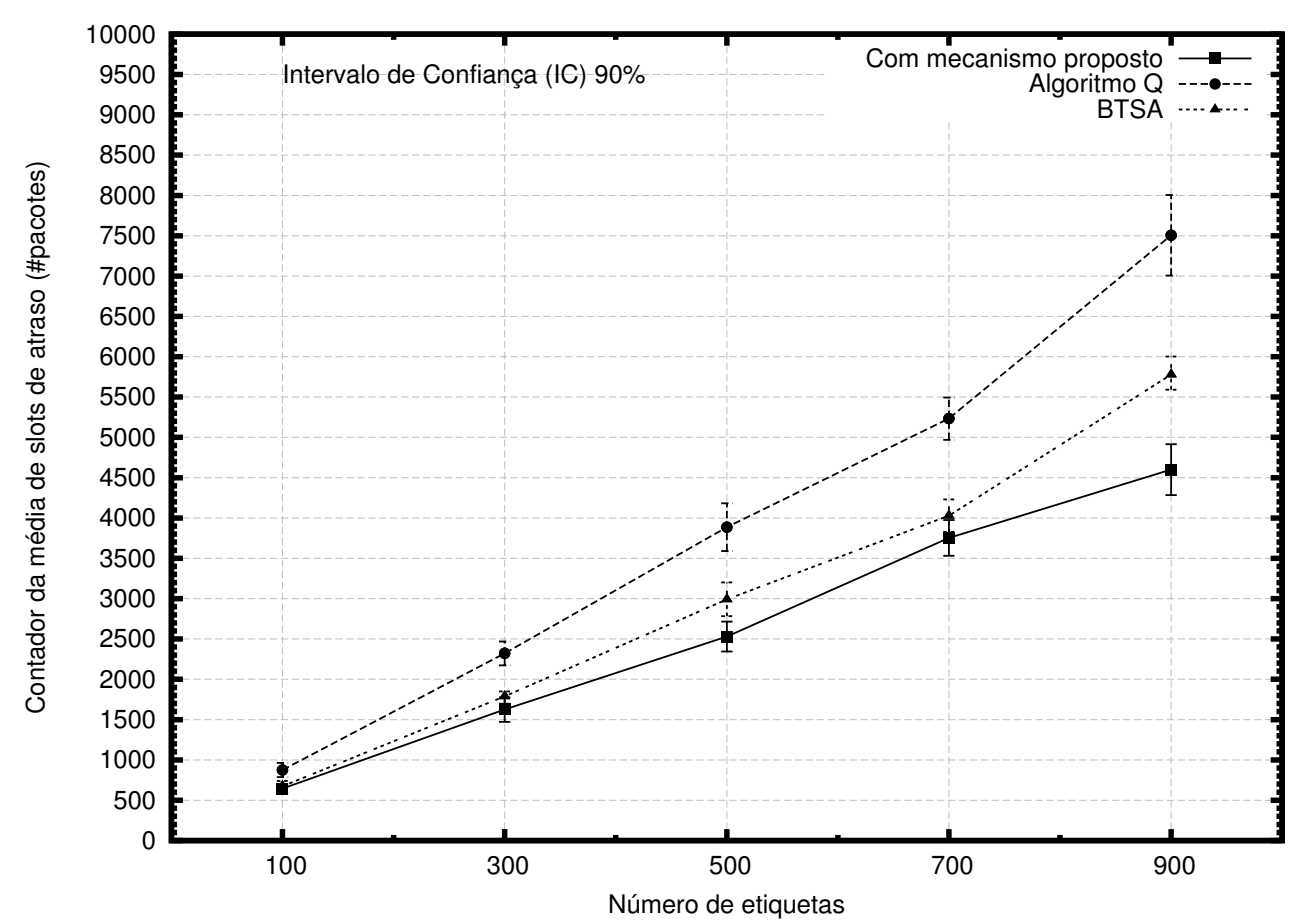

Figura 5.7: Slots de atraso gerados a partir da utilização de diferentes algoritmos/mecanismos

consegue diminuir a taxa de perdas, assim como a quantidade de Kilobytes transferidos na rede. Os padrões de movimentação justificam o padrão constante quando o mecanismo é utilizado. Como não trata-se de um mecanismo anticolisão, as métricas avaliadas não possuem relação com as apresentadas no Capítulo 4.

Dada a crescente utilização de RFID na prática, como por exemplo a utilização em passaportes e uniformes escolares ${ }^{1}$ no Brasil, pode-se observar a importância da pesquisa realizada, visto que os cenários analisados, cada vez mais, farão parte da rotina das pessoas, com grandes quantidades de etiquetas se movimentando e tendo que ser identificadas rapidamente pelos leitores.

Trabalhos científicos sobre o mecanismo foram publicados em (Mota e Batista, 2013; Perazzo Barbosa Mota e Batista, 2013).

\footnotetext{
${ }^{1}$ http://www.dpf.gov.br/servicos/passaporte/passaporte-eletronico/ - Acessado em Agosto de 2015 http://www.congressorfid.com.br/entrevista-edilson/ - Acessado em Agosto de 2015
} 


\subsection{Um Módulo do ns-2 para simular Sistemas RFID Passivos}

No início da pesquisa notou-se a necessidade de um ambiente de simulação para sistemas RFID passivos para que fosse possível avaliar as métricas dos novos mecanismos propostos. O estudo dos softwares existentes (Seção 2.5) mostrou que eles não eram suficientes para as necessidades desta tese porque os mesmos foram desenvolvidos para atender a camada de aplicação e não a camada de acesso ao meio. O foco da implementação do módulo são as abordagens probabilísticas dos mecanismos anticolisão como o Algoritmo Q (EPCglobal, GS1 Inc., 2015), Schoute (Schoute, 1983), Eom-Lee (Eom e Lee, 2010) e um mecanismo para garantia de QoS que será explicado na Seção 5.1.

O módulo foi validado através de comparações com resultados publicados anteriormente. Foram comparados os principais parâmetros pesquisados, baseados no padrão ISO/IEC 18000-6C (EPCglobal, GS1 Inc., 2015), como a Eficiência do Sistema (Sef) e a quantidade total de slots utilizados durante todo o processo de identificação $\left(T_{\text {slots }}\right)$.

\subsubsection{Detalhes do módulo RFID para ns-2}

O módulo foi desenvolvido na versão 2.35 do simulador ns-2. O mesmo foi modelado para seguir não apenas o padrão ISO/IEC 18000-6C, mas também outros algoritmos anticolisão como Schoute (Schoute, 1983) e Eom-Lee (Eom e Lee, 2010) e suas variações. O modelo de mobilidade presente no ns-2 também pode ser utilizado nos cenários RFID. O escopo do módulo baseia-se na parte relacionada ao acesso múltiplo do meio compartilhado. A simulação do processo de identificação foi simplificada a fim de concentrar apenas os pacotes relevantes para avaliação de desempenho dos algoritmos anticolisão, conforme mostrado na Figura 3.4 do Capítulo 3. A Tabela 5.2 mostra as inclusões (C) e modificações (M) de arquivos na estrutura de diretórios do ns-2, enquanto que a Figura 5.8 ilustra o diagrama de classes do módulo proposto. Em resumo, acrescentaram-se três classes principais que modelam os agentes Leitores, Etiquetas e o Pacote RFID trocado entre ambos. Os arquivos modificados mostrados na Tabela 5.2 permitiram a inclusão das classes criadas, assim como seus parâmetros de configuração. 
Tabela 5.2: Arquivos (M)odificados/(C)riados

\begin{tabular}{ccl}
\hline Arquivo & $(\mathbf{C})$ riado/(M)odificado & Comentários \\
\hline apps/rfidReader. $\{\mathbf{c c}, \mathbf{h}\}$ & $\mathrm{C}$ & Definições da classe Leitor \\
apps/rfidTag. $\{\mathbf{c c}, \mathbf{h}\}$ & $\mathrm{C}$ & Definições da classe Etiqueta \\
apps/rfidPacket. $\{\mathbf{c c}, \mathbf{h}\}$ & $\mathrm{C}$ & Definições da classe Pacote \\
trace/cmu-trace.cc & $\mathrm{M}$ & $\begin{array}{l}\text { Inclusão das informações RFID } \\
\text { no arquivo de trace }\end{array}$ \\
tcl/lib/ns-default.tcl & $\mathrm{M}$ & $\begin{array}{l}\text { Inclusão dos parâmetros dos lei- } \\
\text { tores e etiquetas }\end{array}$ \\
common/packet.h & $\mathrm{M}$ & Inclusão do pacote RFID \\
Makefile.in & $\mathrm{M}$ & $\begin{array}{l}\text { Inclusão das classes criadas para } \\
\text { compilação em conjunto com todo } \\
\end{array}$ \\
& & o ns-2 \\
\hline
\end{tabular}
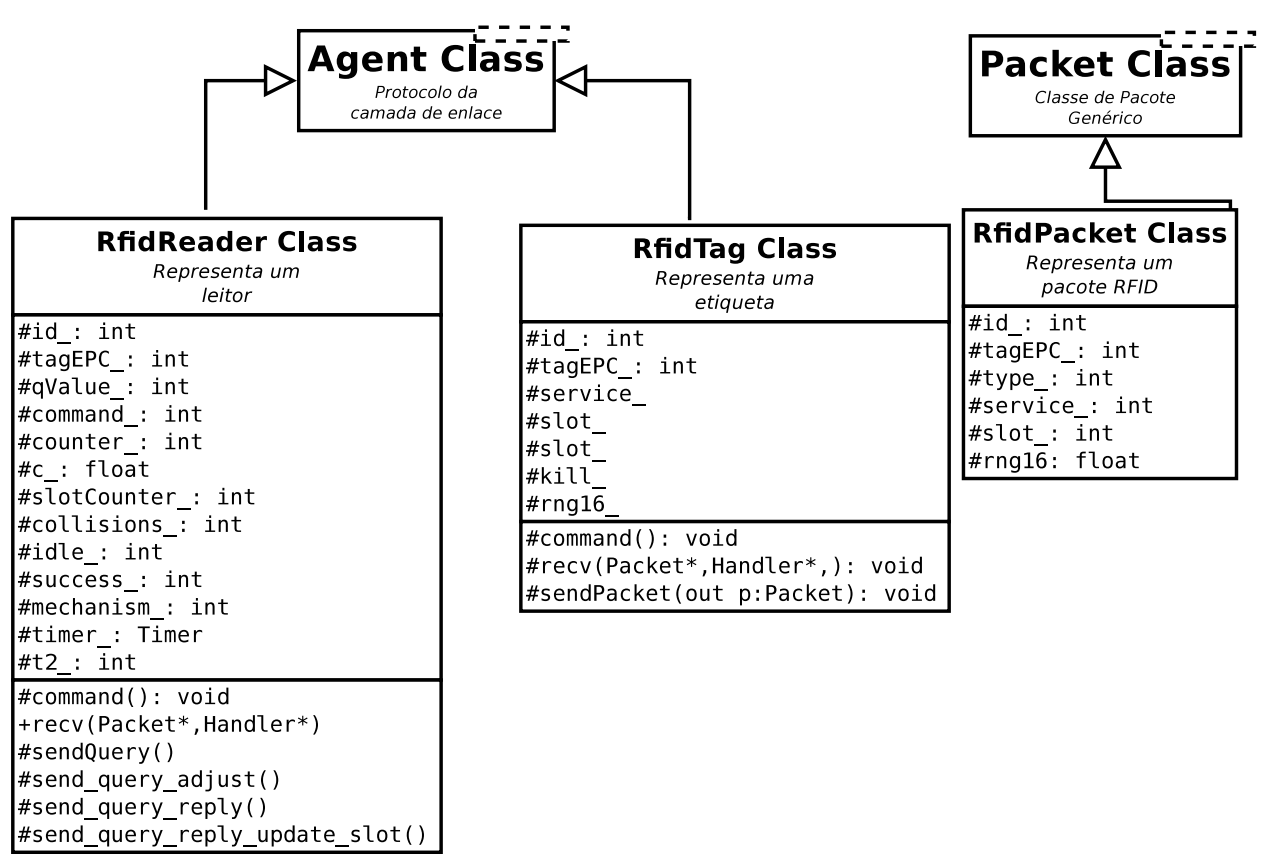

Figura 5.8: Diagrama de Classes do módulo RFID proposto

\section{Agente RfidReader - Leitor}

O Agente que modela um leitor RFID implementa as principais funções relacionadas aos comandos Query utilizados para a identificação de um conjunto de etiquetas, utilizando diferentes algoritmos anticolisão propostos. Os principais parâmetros das propostas podem também ser alterados visando uma completa exploração de cada uma das propostas existentes. Entre estes parâmetros pode-se citar o tamanho inicial do quadro, valor inicial da variável Q, entre outros. A implementação foi realizada utilizando-se seis grupos de variáveis conforme mostrado na Figura 5.8:

- Identificação: Armazena a identificação do leitor e etiquetas (id_e tagEPC_); 
- Parâmetros relacionados ao padrão ISO/IEC 18000-6C: Trata de todos os parâmetros relacionados ao algoritmo Q como o valor de Q (qValue_), tipo de comando (command_ $)$, contador de respostas (counter_) e tempo de espera (t_2_);

- Contadores: Utilizados para contabilizar todos os tipos de slots como as colisões (collisions_), sucessos (success_), vazios (idle_) e total (slotCounter_);

- métodos padrão do protocolo ISO/IEC 18000-6C: Implementação dos comandos Query, QueryAdjust e QueryRep utilizados para identificar a população de etiquetas (send_);

- Métodos de envio e recepção: Utilizado para implementar o envio e recepção de pacotes $(\operatorname{recv}()$ e command ()$)$;

- Comandos de QoS: Implementados para prover mecanismos de Qualidade de Serviço (mechanism_e timer_).

\section{Agente RfidTag - Etiqueta}

O Agente que implementa as etiquetas foi modelado para operar de forma passiva, sendo assim impossibilitado de iniciar uma comunicação com os leitores. A estrutura interna implementada inclui os recursos básicos como gerador de números aleatórios, memória opcional e estado, assim como recursos adicionais como memória destinada à implementação de QoS.

\section{Agente RfidPacket - Pacote}

Esta classe foi desenvolvida de forma a facilitar a análise do arquivo de trace gerado, uma vez que o trace é gerado a partir de informações extraídas da troca de pacotes entre leitores e etiquetas. O pacote RFID modelado provê todas as informações relacionadas aos parâmetros necessários para a correta análise dos protocolos anticolisão como total de slots utilizados, quantidades parciais de slots de colisão, vazio e sucesso assim como tipo de comando e direção do fluxo de cada mensagem. A modelagem visa permitir que os utilizadores possam analisar o comportamento dos algoritmos anticolisão sob diferentes aspetos, principalmente com relação ao desempenho, visto que o mesmo é analisado através da quantidade e tipos de slots gerados. 


\section{Arquivo de trace}

O arquivo de trace é um dos recursos mais importantes do ns-2, pois o mesmo permite a análise posterior da simulação. A Tabela 5.3 mostra o formato do arquivo de trace gerado após a simulação de um cenário RFID. Todos os itens da tabela precisaram ser incluídos no módulo proposto.

Tabela 5.3: Formato do arquivo de trace

\begin{tabular}{|c|c|c|}
\hline Flag & Valor & Comentários \\
\hline $\mathbf{s} / \mathbf{r}$ & Envio (s) ou recepção (r) & $\begin{array}{l}s \text { indica o envio de um pacote e } r \text { a recepção } \\
\text { de um pacote }\end{array}$ \\
\hline$-\mathrm{t}$ & Tempo do evento & Tempo exato da ocorrência do evento \\
\hline$-\mathrm{Zt}$ & Tipo de fluxo & $\begin{array}{l}0 \text { representa o fluxo leitor para etiqueta e } 1 \\
\text { etiqueta para leitor }\end{array}$ \\
\hline$-\mathrm{Zi}$ & ID do nó & $\begin{array}{l}\text { Identificador (ID) da etiqueta ou leitor que } \\
\text { está enviando/recebendo o pacote }\end{array}$ \\
\hline$-\mathrm{Zs}$ & Nó origem & Identifica quem enviou o pacote \\
\hline$-\mathrm{Zd}$ & Nó destino & Identifica quem recebeu o pacote \\
\hline$-\mathrm{Zc}$ & Tipo de comando & $\begin{array}{l}0 \text { - Query; } 1 \text { - QueryAdjust; } 2 \text { - QueryRep; } 3 \text { - } \\
\text { Reader ACK; } 4 \text { - Reader NAK; } 5 \text { - Resposta } \\
\text { da etiqueta }\end{array}$ \\
\hline$-\mathrm{Zq}$ & Tipo de serviço & $\begin{array}{l}\text { Representa o tipo de comunicação que está } \\
\text { sendo realizado: } 2 \text { - Algoritmo Q puro; } 3 \text { - } \\
\text { Algoritmo Q puro com QoS Seção } 5.14 \text { - } \\
\text { Schoute; } 5 \text { - Eom-Lee }\end{array}$ \\
\hline$-\mathrm{Zr}$ & Número aleatório gerado & $\begin{array}{l}\text { Número aleatório gerado pelas etiquetas ba- } \\
\text { seado na fórmula: } r=\operatorname{random}\left(0,2^{Q}-1\right)\end{array}$ \\
\hline$-\mathrm{Zv}$ & Valor de Q & Valor atual de Q do leitor \\
\hline$-\mathrm{Zz}$ & Contador de slots & $\begin{array}{l}\text { Conta o número total de slots incluindo co- } \\
\text { lisões, vazios e sucessos }\end{array}$ \\
\hline$-\mathrm{Zc}$ & Contador de slots de colisão & $\begin{array}{l}\text { Conta o número total de colisões geradas du- } \\
\text { rante todo o processo de identificação }\end{array}$ \\
\hline$-\mathrm{Zi}$ & Contador de slots vazios & $\begin{array}{l}\text { Conta o número total de slots vazios gerados } \\
\text { durante todo o processo de identificação }\end{array}$ \\
\hline$-\mathrm{Zs}$ & Contador de slots de sucesso & $\begin{array}{l}\text { Conta o número total de slots de sucesso ge- } \\
\text { rados durante todo o processo de identifica- } \\
\text { ção }\end{array}$ \\
\hline$-\mathrm{Ss}$ & ID da sessão & $\begin{array}{l}\text { Número da sessão (cada novo comando } \\
\text { Query gera uma nova sessão) }\end{array}$ \\
\hline
\end{tabular}

\subsubsection{Experimentos}

Foram conduzidos dois conjuntos de experimentos para a avaliação do módulo. O primeiro visou verificar a adequação ao padrão definido em (EPCglobal, GS1 Inc., 2015). As 
simulações avaliaram o funcionamento do Algoritmo Q através dos parâmetros mais avaliados na literatura (Baloch e Pendse, 2013; Li et al., 2009; Namboodiri et al., 2012; Wang et al., 2007), como $S_{\text {ef }}$ e $T_{\text {slots }}$. O segundo conjunto de experimentos teve como objetivo comparar o mecanismo de QoS apresentado na Seção 5.1 com o Algoritmo Q puro e a proposta de Wu et al. (2013). As Equações 5.3, 5.4 e ?? mostram como foram calculados os valores médios para cada uma das medições. Nos dois primeiros casos foram realizados 100 experimentos e calculados a média e o intervalo de confiança. A Equação 5.3 calcula a média de 100 medições do parâmetro $S_{e f}$, sendo cada uma das medições é a razão entre a quantidade de etiquetas identificadas pelo total de slots utilizados durante todo o processo de identificação. O parâmetro $T_{\text {slots }}$ também é calculado através da média das 100 medições realizadas, onde cada medição é a soma simples da quantidade de slots utilizados.

$$
\begin{aligned}
S_{\text {ef }}= & \frac{\sum_{i=1}^{100} \frac{s u c_{i}}{s u c_{i}+c o l_{i}+i d l_{i}}}{100} \\
T_{\text {slots }}= & \frac{\sum_{i=1}^{100} s u c_{i}+\operatorname{col}_{i}+i d l_{i}}{100}
\end{aligned}
$$

onde

- $i$ - Iteração;

- $c o l_{i}$ - Número de slots de colisão da iteração $i$;

- $i d l_{i}$ - Número de slots vazios da iteração $i$;

- $s u c_{i}-$ Número de slots de sucesso da iteração $i$;

- time $_{i}$ - Tempo de identificação (em $\left.m s\right)$.

Os parâmetros de simulação para o cálculo das Equações 5.3 e 5.4 são mostrados na Tabela 5.4.

O segundo conjunto de experimentos introduz o conceito de Slots de Atraso - (Delay Slots)), definido pela Equação 5.5. Este parâmetro representa a soma dos slots que atrasam 
Tabela 5.4: Parâmetros do primeiro conjunto de simulações

\begin{tabular}{cl}
\hline Parâmetro & Valor \\
\hline Cenário & Identificação de um conjunto de etiquetas \\
Tempo de simulação & 10 segundos \\
Número de etiquetas & 50 até 1850 \\
Passos & De $50 \mathrm{em} 50$ \\
Número de leitores & 1 \\
Posição inicial das etiquetas & Aleatório (dentro da zona de cobertura do leitor) \\
Movimentação & Sem movimentação \\
Quantidade de solicitações do leitor & Uma solicitação \\
Classe geradora de números aleatórios & Random::uniform $\left(0,2^{q}-1\right)$ \\
Valor Q inicial & 4 \\
Constante $c$ & 0.3 \\
Tempo de espera por respostas $\left(T_{2}\right)$ & 1 ms (de acordo com Namboodiri et al. (2012)) \\
Número de Iterações $S_{\text {ef }}$ e $T_{\text {slots }}$ & 100 \\
\hline
\end{tabular}

o processo de identificação, ou seja, os vazios e de colisão, para cada um dos leitores do cenário. Este parâmetro foi avaliado para verificar a redução dos mesmos quando aplicado o mecanismo proposto na Seção 5.1, comparado com o Algoritmo Q Puro e o BTSA de Wu et al. (2013). A relevância da análise deste parâmetro está relacionada ao consumo de energia por parte dos leitores, uma vez que este consumo está diretamente relacionado com a quantidade de slots de atraso presentes na comunicação entre leitores e etiquetas (Klair et al., 2009). Os parâmetros utilizados neste conjunto de simulações são apresentados na Tabela 5.5.

$$
T_{\text {delay }}=\sum_{i=1}^{r} \sum_{j=1}^{m}\left(\operatorname{col}_{i, j}+i d l_{i, j}\right)
$$

onde

- $i$ - Leitor $i$;

- $r$ - Número de Leitores;

- $j$ - Sessão (requisição) $j$;

- $m$ - Número de sessões durante toda a simulação;

- $\operatorname{col}_{i, j}$ - Número de slots de colisão do leitor $i$ durante a sessão $j$; 
- $i d l_{i, j}$ - Número de slots vazios do leitor $i$ durante a sessão $j$;

- $T_{\text {delay }}$ - Número total de slots de atraso durante toda a simulação;

Tabela 5.5: Parâmetros do segundo conjunto de simulações

\begin{tabular}{cl}
\hline Cenário & 3 compartimentos com um leitor cobrindo cada um deles \\
Tempo de simulação & 1800 segundos \\
Quantidade de etiquetas & 100 até 900 \\
Número de Leitores & 3 \\
Passos & de 200 em 200 \\
Posição inicial & Aleatória (0-30 metros x 0-15 metros) \\
Padrão de movimentação & Random Way Point ${ }^{2}(0-30$ metros x 0-15 metros) \\
Quantidade de requisições & Cada leitor envia uma solicitação por minuto \\
Topologia & $30 x 15$ (metros) \\
Velocidade dos nós & $0-1,5$ (metros/segundo) \\
\hline
\end{tabular}

Ambos conjuntos de simulações foram realizadas em um servidor equipado com processador Intel Core i7-2700K 3,5GHz, 16GB de RAM e 1TB de espaço em disco, executando o sistema operacional Debian GNU / Linux versão 6.0.

\subsubsection{Resultados e Validação}

Esta seção apresenta os resultados das simulações utilizadas para a validação do módulo RFID proposto. A Figura 5.9 mostra a medida do desempenho do sistema $\left(S_{e f}\right)$ comparada com outros resultados publicados. A curva do módulo proposto encontra-se entre a medida mínima de Sanchez M e Ramos R (2012) e a medida máxima de Wu et al. (2013), apresentando valor aproximado de $\cong 0.32$. O aspecto importante a ser observado é a característica próxima de um valor constante da curva. As variações devem-se principalmente à função geradora de números aleatórios utilizada por cada uma das simulações. Nem todas as curvas apresentam resultados para o intervalo do número de etiquetas entre 50 e 1850, pois algumas publicações utilizaram intervalos diferentes. Dessa forma as curvas estão plotadas em intervalos diferentes umas das outras.

A Figura 5.10, assim como a Figura 5.9, apresenta resultados importantes para validação do módulo. A principal característica desta curva, que mede a quantidade total de pacotes trocados durante a identificação, segundo Chen (2012), Sanchez M e Ramos R (2012) e Baloch e Pendse (2013) é o aspecto linear da mesma. Pode-se observar que a curva do 


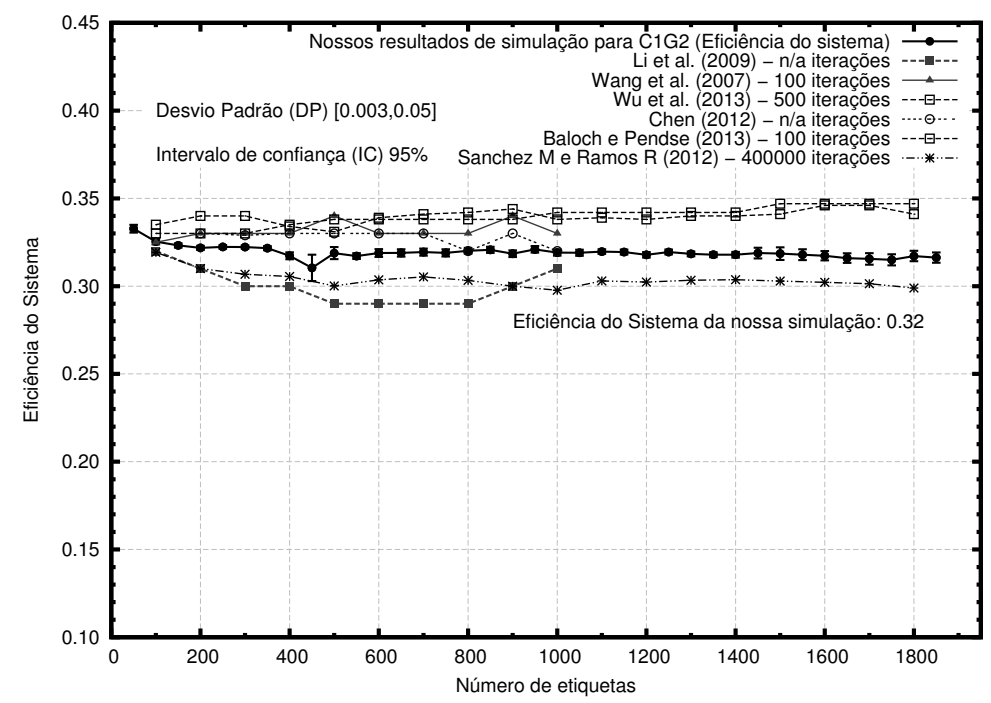

Figura 5.9: Comparação de resultados para eficiência do sistema $\left(S_{\text {ef }}\right)$

módulo proposto apresenta esta aparência, assim como as demais apresentadas. Este fato, juntamente com o aspecto próximo do constante do gráfico anterior comprova a fidelidade dos resultados do módulo com outros resultados apresentados na literatura.

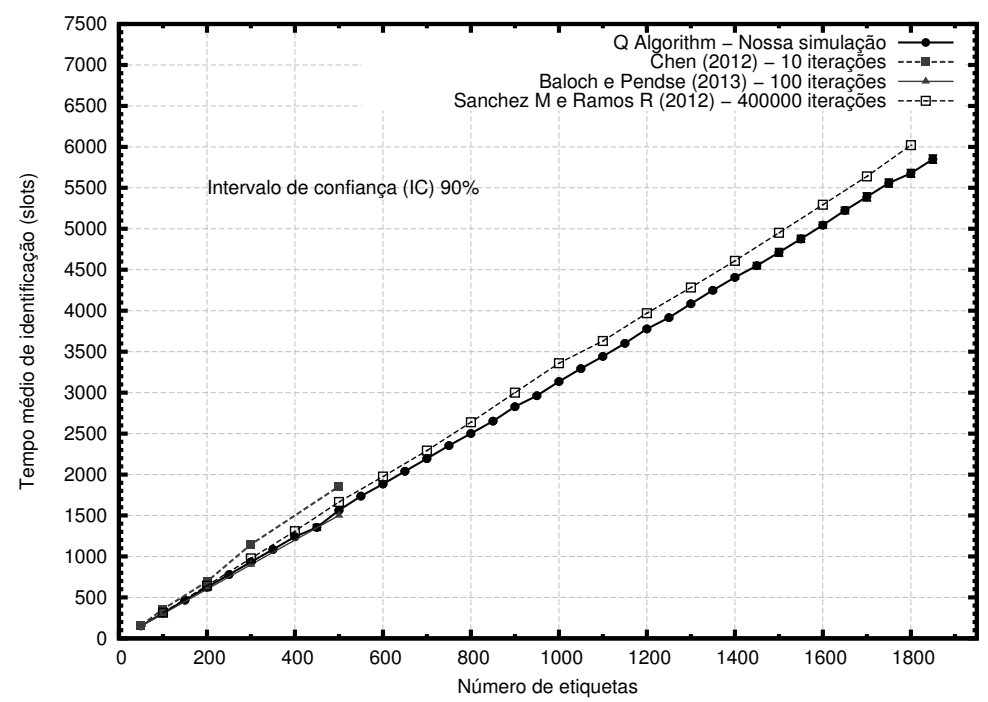

Figura 5.10: Comparação de resultados para o total de slots $\left(T_{\text {slots }}\right)$

Finalmente a Figura 5.11 apresenta os resultados obtidos quando aplicado o mecanismo de QoS proposto na Seção 5.1, comparado com a utilização do Algoritmo Q Puro e com o BTSA de Wu et al. (2013). Pode-se observar que a aplicação do mecanismo em um cenário de rastreamento e localização obtêm ganhos relevantes mesmo quando comparados com o BTSA. O ganho obtido pode variar de $10 \%$ a $40 \%$ e $6 \%$ a $37 \%$ quando comparado ao Algoritmo Q padrão e ao BTSA respectivamente, quando o número de etiquetas varia de 
100 a 900.

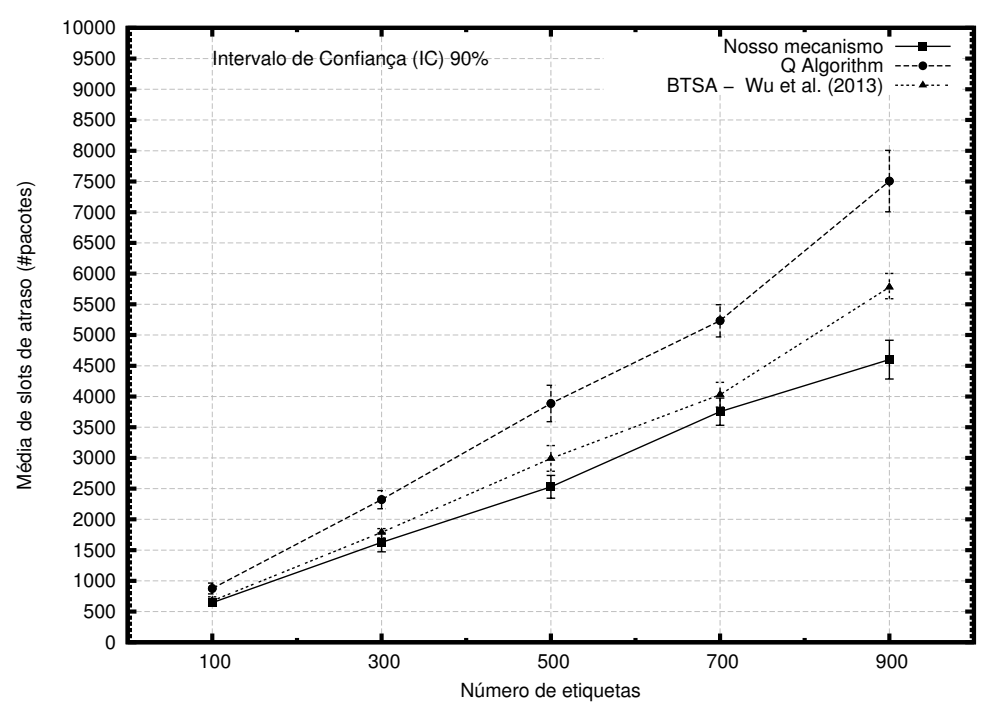

Figura 5.11: Comparação de resultados com e sem a aplicação do mecanismo de QoS

Em resumo, os resultados apresentados nesta seção foram obtidos para validação do módulo desenvolvido e os mesmos confirmam algumas observações apresentadas na literatura (Wu et al., 2013) (Sanchez M e Ramos R, 2012) (Baloch e Pendse, 2013) (Chen, 2012):

- $T_{\text {slots }}$ apresenta curva com característica linear quando o número de etiquetas aumenta;

- $S_{\text {ef }}$ possui aspecto próximo de valor constante, mesmo quando aumentamos a quantidade de etiquetas.

\subsubsection{Conclusões parciais}

Esta seção apresentou um novo módulo RFID para o simulador ns-2. O módulo introduz uma plataforma de simulação para desenvolvimento de cenários RFID. O módulo implementa a parte anticolisão do protocolo ISO/IEC 18000-6C para etiquetas passivas e aproveita o modelo de mobilidade já presente no ns-2. O mesmo também suporta outros algoritmos DFSA como Schoute e Eom-Lee. Por ter sido projetado como uma extensão ao ns-2, sua possibilidade de expansão é facilitada, uma vez que várias outras tecnologias sem fio já encontram-se implementadas nativamente no ns-2. Experimentos foram realizados para avaliar a implementação da parte anticolisão do protocolo ISO/IEC 18000-6C. Os resultados indicam que o módulo funciona de acordo com os requerimentos do padrão produzindo 
resultados similares a outros resultados mostrados na literatura tanto por via de simulação quanto com resultados analíticos. O módulo proposto pode beneficiar pesquisadores nas áreas de redes RFID, especialmente aqueles que desejam aprofundar-se em QoS.

O módulo, batizado de nsRFIDSim está disponível sob a licença GPL no sítio do Centro de Competência em Software Livre - $\mathrm{CCSL}^{3}$ do Instituto de Matemática e Estatística da USP. Um trabalho científico sobre ele foi publicado em (Perazzo Barbosa Mota e Batista , 2013).

${ }^{3}$ http://ccsl.ime.usp.br/pt-br/project/nsrfidsim-0. Acessado em Agosto de 2015. 


\subsection{Um algoritmo DFSA estimado: NEDFSA}

Nesta seção é apresentado um algoritmo anticolisão para sistemas RFID, chamado NEDFSA (Novel Estimated Dynamic Frame Slotted Aloha). Através de resultados de simulação, com a utilização do módulo para o simulador ns-2 apresentado na Seção 5.2, observou-se que o NEDFSA melhora em 14\% a eficiência do sistema em relação ao algoritmo Q. Uma das principais vantagens do NEDFSA é que poucas modificações necessitam ser realizadas para implementação prática. A eficiência do NEDFSA não é apenas melhor que as principais propostas existentes, mas também equivalente à proposta de Wu et al. (2013) que é reconhecidamente eficiente.

Em resumo, nesta seção são apresentadas:

- A proposta de um novo algoritmo anticolisão baseado em DFSA, o NEDFSA, que aumenta a eficiência do sistema em até 14\% em comparação ao Algoritmo Q;

- Uma análise abrangente dos vários parâmetros utilizados pelas propostas existentes, como quantidade de colisões por slot, tamanho inicial de quadros, e estimativa do número de etiquetas presentes na área de cobertura do leitor.

As principais vantagens do NEDFSA estão na estimativa inicial eficiente da quantidade de etiquetas, tendo como base uma potência de 2, similar ao realizado pelos algoritmos BTSA, o fato de lidar com colisões no momento em que elas acontecem.

\subsubsection{Algoritmo proposto}

Como o algoritmo proposto baseia-se em uma estimativa inicial da quantidade de etiquetas, o mesmo inicia sua operação fazendo este cálculo, de acordo com o fluxograma mostrado na Figura 5.12. O primeiro passo zera os contadores de slots de colisão (collisions), sucesso (success) e vazios (idle). O passo seguinte faz o envio de $i$ solicitações do comando QueryEst, que visa verificar o tipo do slot de tempo (colisão, sucesso ou vazio). Caso a quantidade de slots de colisão ou vazios seja igual a $i$, aumenta-se ou diminui-se o valor de Q em $c$ unidades e o procedimento é reiniciado com este novo valor de Q. Caso contrário, uma nova rodada 
de envio de $i$ comandos QueryEst é realizada para confirmar se o valor de Q está adequadamente estimando a quantidade de etiquetas. Caso a confirmação seja positiva (colisões e vazios diferente de $i$ ) estima-se a quantidade de etiquetas como $2^{Q}$. Senão aumenta-se ou diminui-se o valor de $\mathrm{Q}$ em $c$ unidades e todo o processo é reiniciado. Antes da continuação do projeto do algoritmo NEDFSA, analisou-se o comportamento do procedimento de estimativa sob diferentes valores de $i$ e $c$.

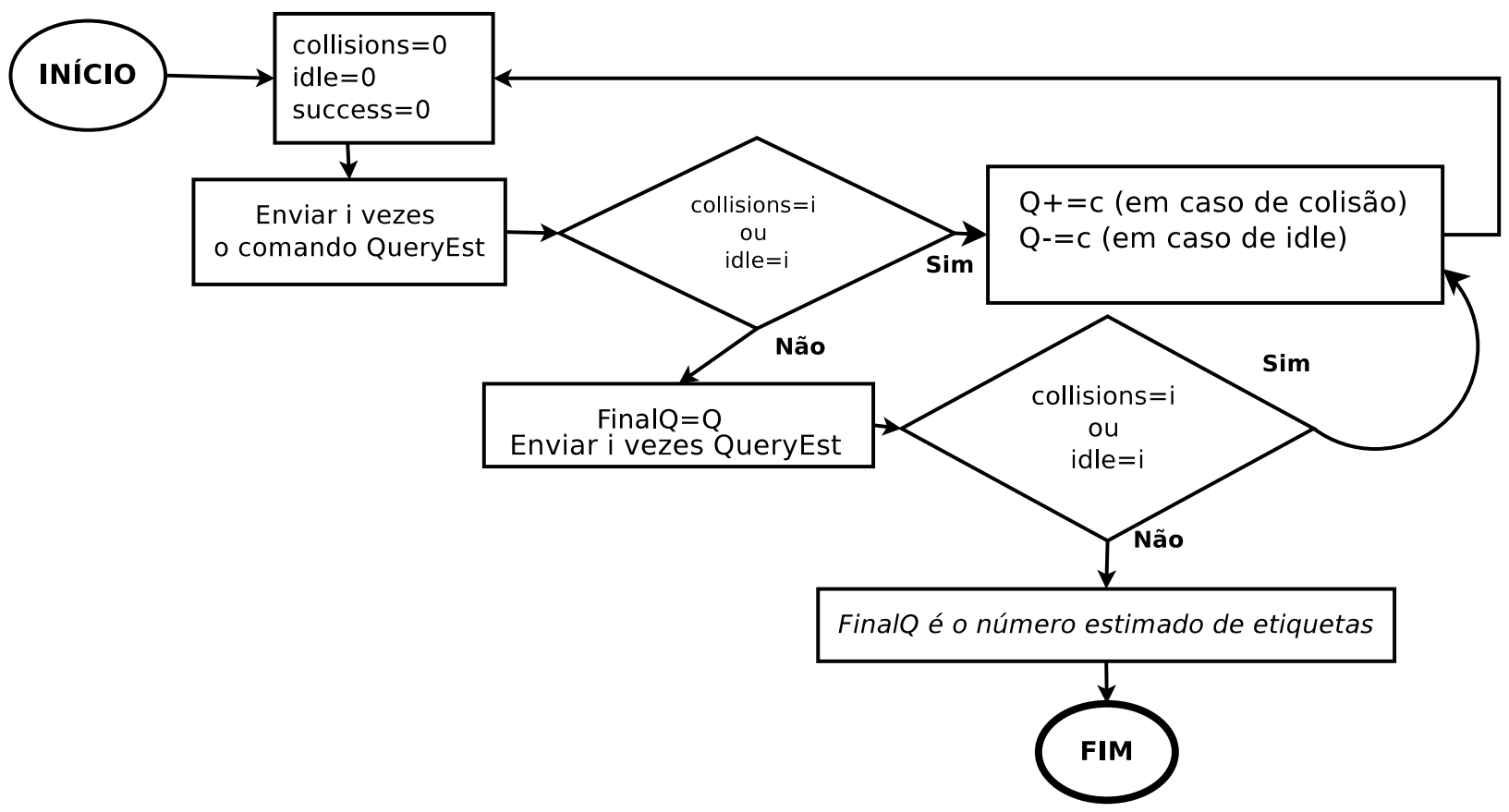

Figura 5.12: Procedimento de estimativa

Segundo Cui e Zhao (2009), os valores ideais para $i$ e $c$ são 3 e 1 respectivamente. Foram realizados experimentos de simulação para verificar estes valores. Os resultados são apresentados nas Figuras 5.13 e 5.14. Na Figura 5.13 são mostrados os resultados de estimativas do número de etiquetas dos pares de parâmetros $i$ e $c$. Por exemplo, quando a quantidade de etiquetas é igual a 100, todas as curvas estimam o valor de Q entre 6 e 7 (entre 64 e 128 etiquetas). Já quando a quantidade de etiquetas cresce, por exemplo a partir de 1600, as curvas referentes ao par $(i, c)$ de valores $(3,1)$ e $(5,1)$ tendem a estimar Q em 11 (2048 etiquetas) enquanto as demais curvas tendem a estimar Q em 10 (1024 etiquetas). Ou seja, no primeiro caso estima-se mais etiquetas e no segundo menos etiquetas.

A estimativa de menos etiquetas do que o número real gera um número maior de colisões a serem resolvidas. Além disso, o overhead de mensagens trocadas durante a estimativa é maior principalmente quando a quantidade de etiquetas é pequena (por exemplo de 100 a 


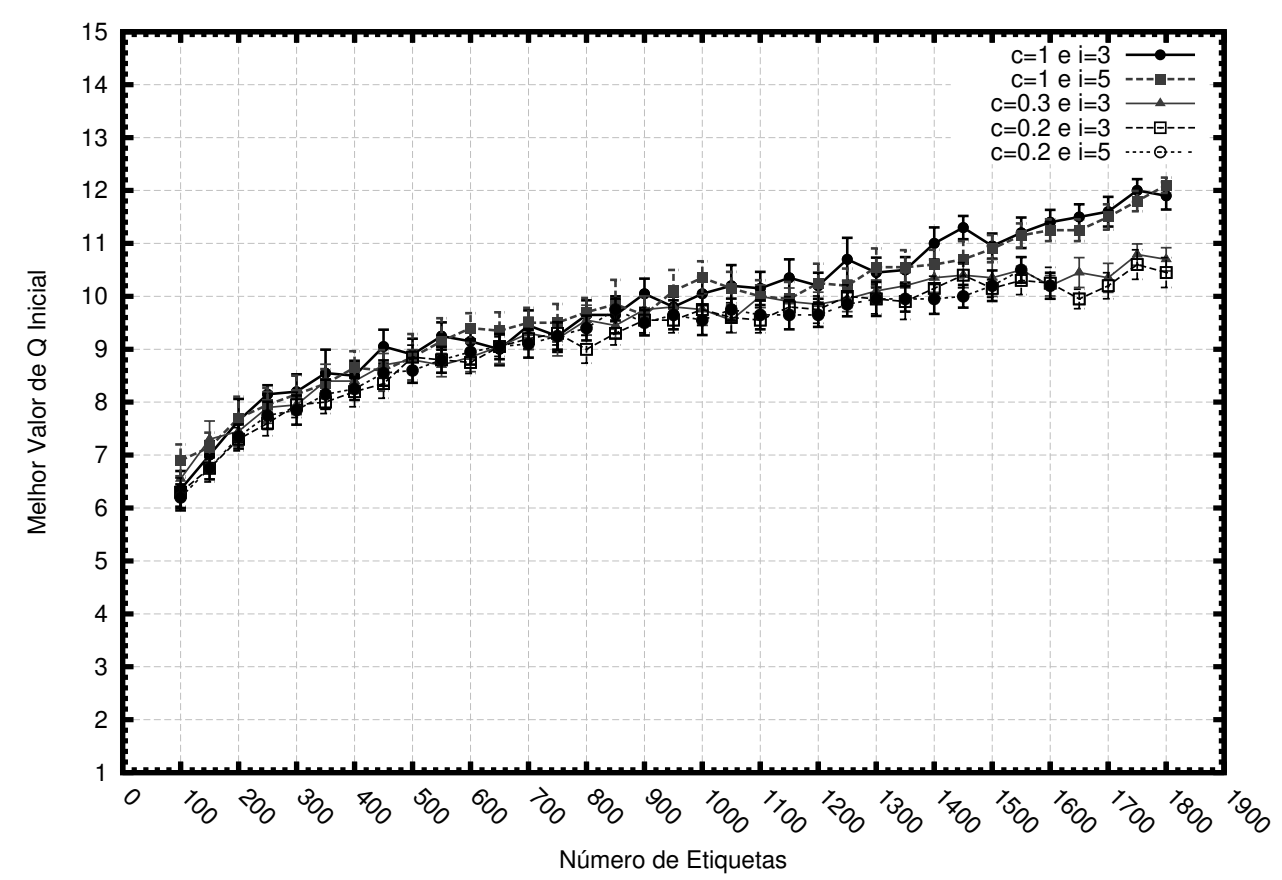

Figura 5.13: Melhor valor inicial de $Q$ por número de etiquetas para diferentes valores de $i$ e $c$

1000). A Figura 5.14 mostra o overhead gerado pela estimativa com a utilização de diferentes valores para $i$ e $c$. Quando temos 300 etiquetas, por exemplo, o overhead da estimativa é de aproximadamente 16\%. Este número significa que a estimativa gera aproximadamente 16\% a mais de mensagens trocadas durante todo o processo de identificação. Já quando o número de etiquetas é 1800 observa-se que o overhead de todos os pares de parâmetros fica abaixo de $3 \%$. A partir do overhead gerado e do valor estimado, decidiu-se utilizar para o NEDFSA os parâmetros propostos por Cui e Zhao (2009) de $i=3$ e $c=1$. Com estes valores tem-se overhead menor e estima-se uma quantidade maior de etiquetas, independente do número real de etiquetas, o que gera menos colisões a serem resolvidas.

O NEDFSA opera inicialmente estimando o valor inicial do número de etiquetas, como uma potência de 2, conforme ilustra a Figura 5.15 e o Algoritmo 7.

Após a estimativa do tamanho inicial do quadro $(L)$, com parâmetros $(i, c)$ igual a $(3,1)$ (linha 1), definem-se os contadores de respostas recebidas (linha 3), quantidade de colisões (linha 4) e slot inicial (linha 2). Como os parâmetros $i=3$ e $c=1$ estimam quantidades maiores de etiquetas, multiplicou-se o valor de $L$ pela constante 0,67 para minimizar os efeitos da estimativa em valores mais altos, reduzindo assim a quantidade de slots a serem percorridos. Posteriormente, o laço das linhas 5-15, realiza a requisição de respostas para 


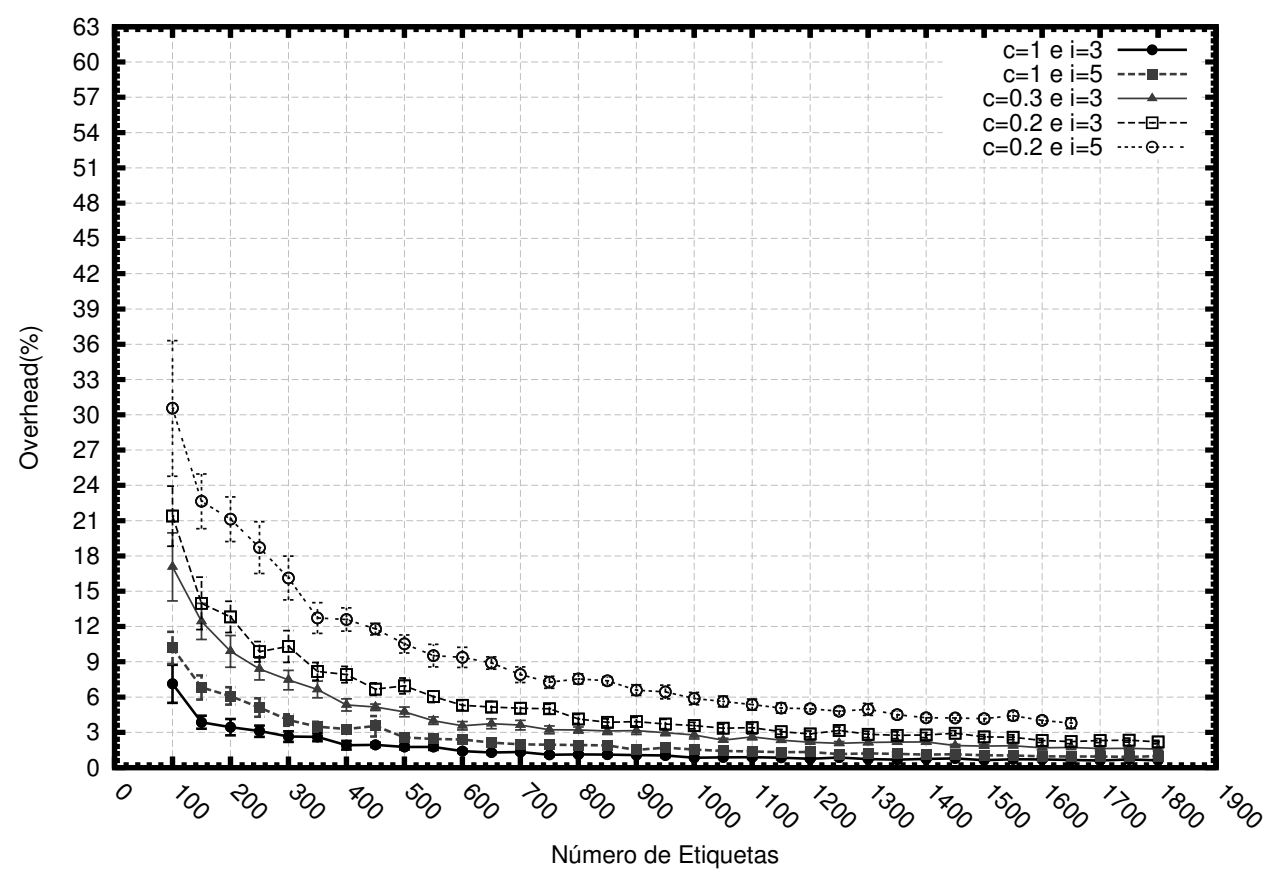

Figura 5.14: Sobrecarga do procedimento de estimativa para diferentes valores de $i$ e $c$

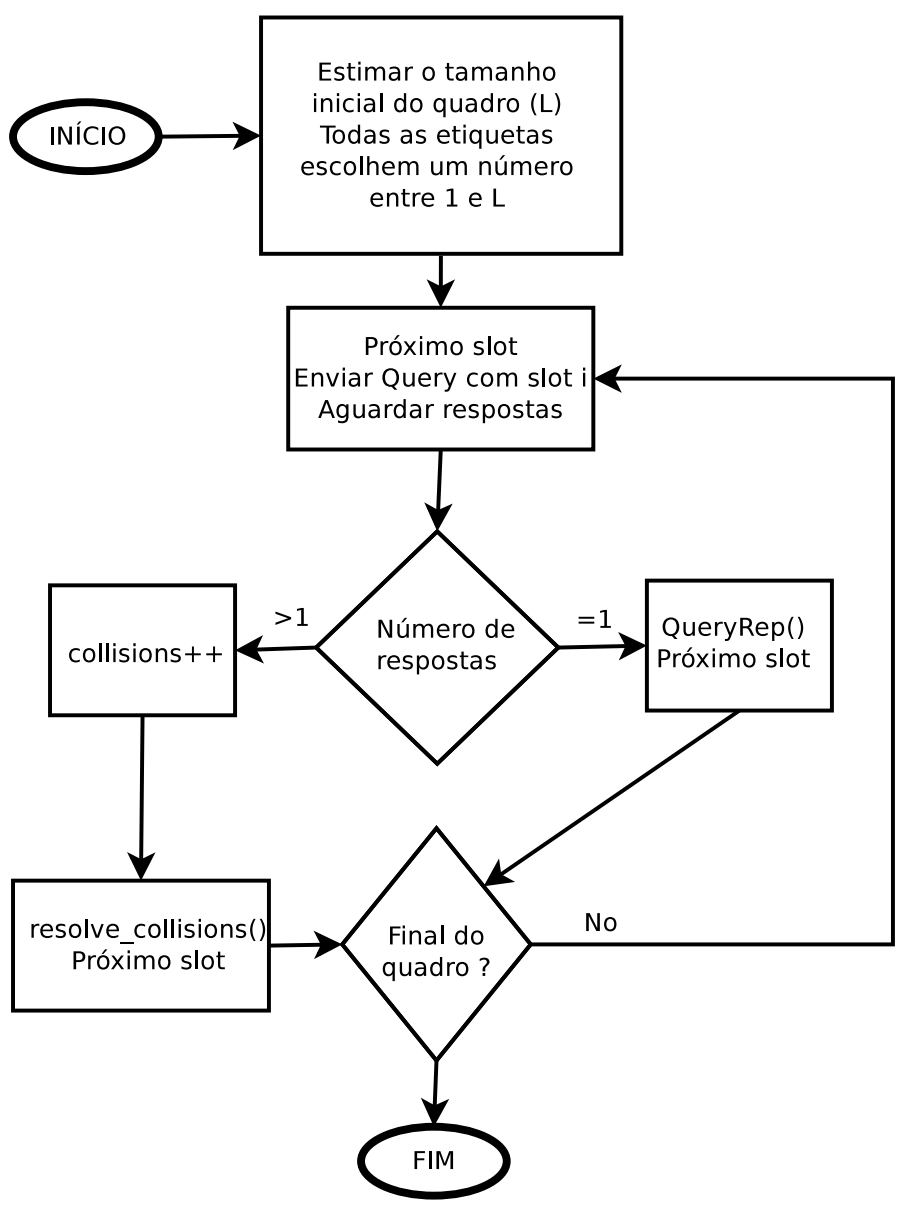

Figura 5.15: Proposta NEDFSA

cada um dos slots, iniciando em 1 e terminando em $L$. Em cada slot o leitor envia um comando Query (linha 6) e espera respostas (linha 7). Caso apenas uma etiqueta responda, 


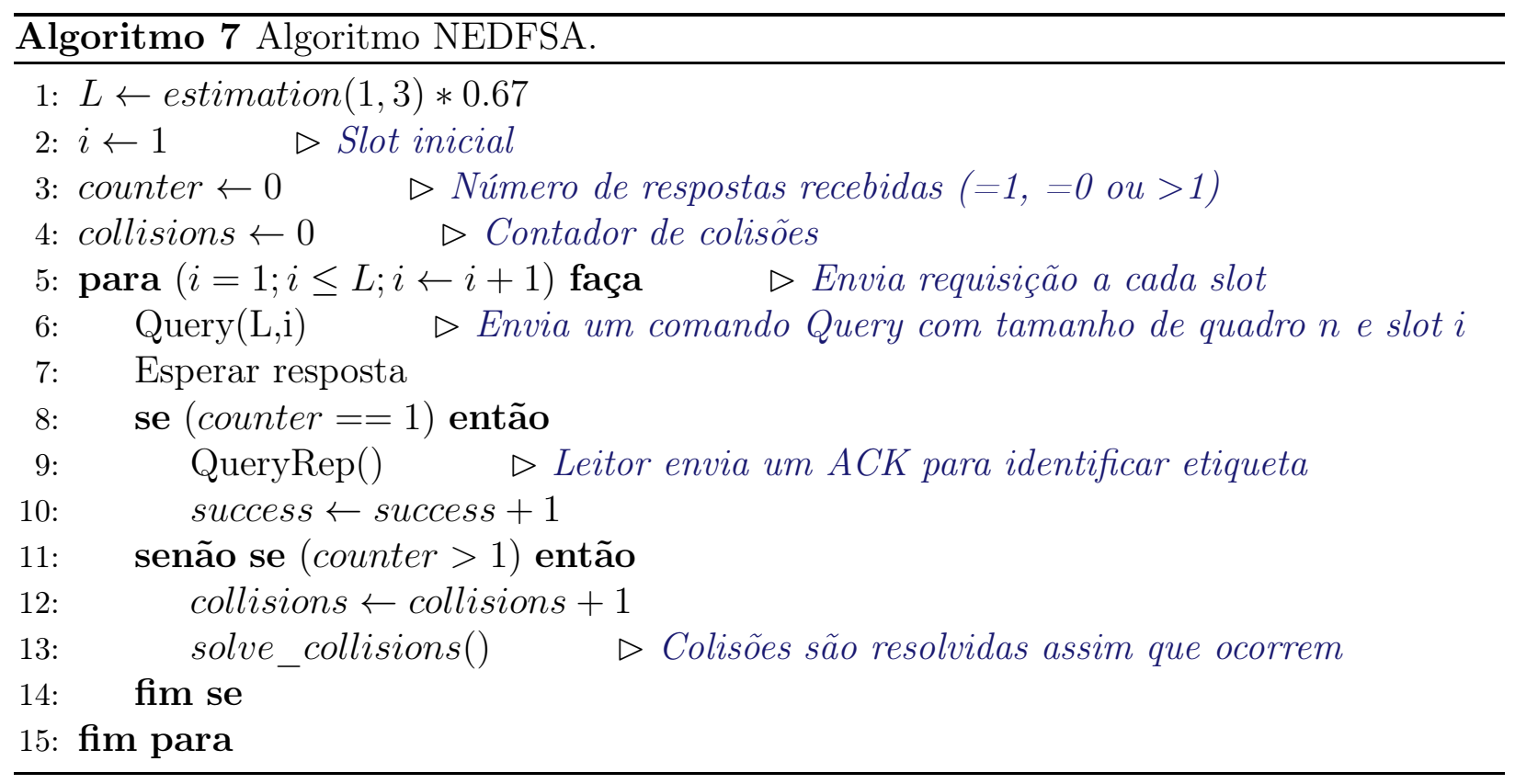

o leitor faz a identificação da mesma (linhas 8-10). Caso mais de uma etiqueta responda, é chamado o procedimento solve_collisions() (linhas 11-13) que será descrito posteriormente. Após a resolução das colisões no slot atual, o algoritmo passa para o próximo slot e repete o procedimento.

Antes da definição do algoritmo de resolução de colisões, foram realizados experimentos para verificação da quantidade média de respostas por slot de colisão. A Figura 5.16 mostra os percentuais da quantidade de etiquetas que disputam um slot nas quase 89000 observações. Mais de $85 \%$ dos slots de colisão ocorreram entre 2 ou 3 etiquetas.

A partir do resultado obtido anteriormente, foram realizados novos experimentos, para resolução de colisões, quando a quantidade de etiquetas em colisão varia de 2 a 8 , utilizandose diferentes algoritmos DFSA propostos, conforme mostrado na Figura 5.17.

Em todos os casos, independente da quantidade de etiquetas em colisão, o Algoritmo Q possui desempenho inferior. Quando temos 2 ou 3 etiquetas em colisão, os melhores algoritmos são o de Eom-Lee e Mota. Para a quantidade maior que 3 etiquetas em colisão tem-se praticamente desempenhos simulares entre as propostas. Para utilização junto ao NEDFSA escolheu-se a proposta de Mota, pois a mesma possui desempenho equivalente à de Eom-Lee e com a vantagem de não incluir rotinas iterativas para obtenção do valor do tamanho do próximo quadro. A proposta para cálculo do backlog de Mota é similar a de Schoute, apresentada na Seção 3.3 com a diferença que Mota utiliza a constante 2.62 


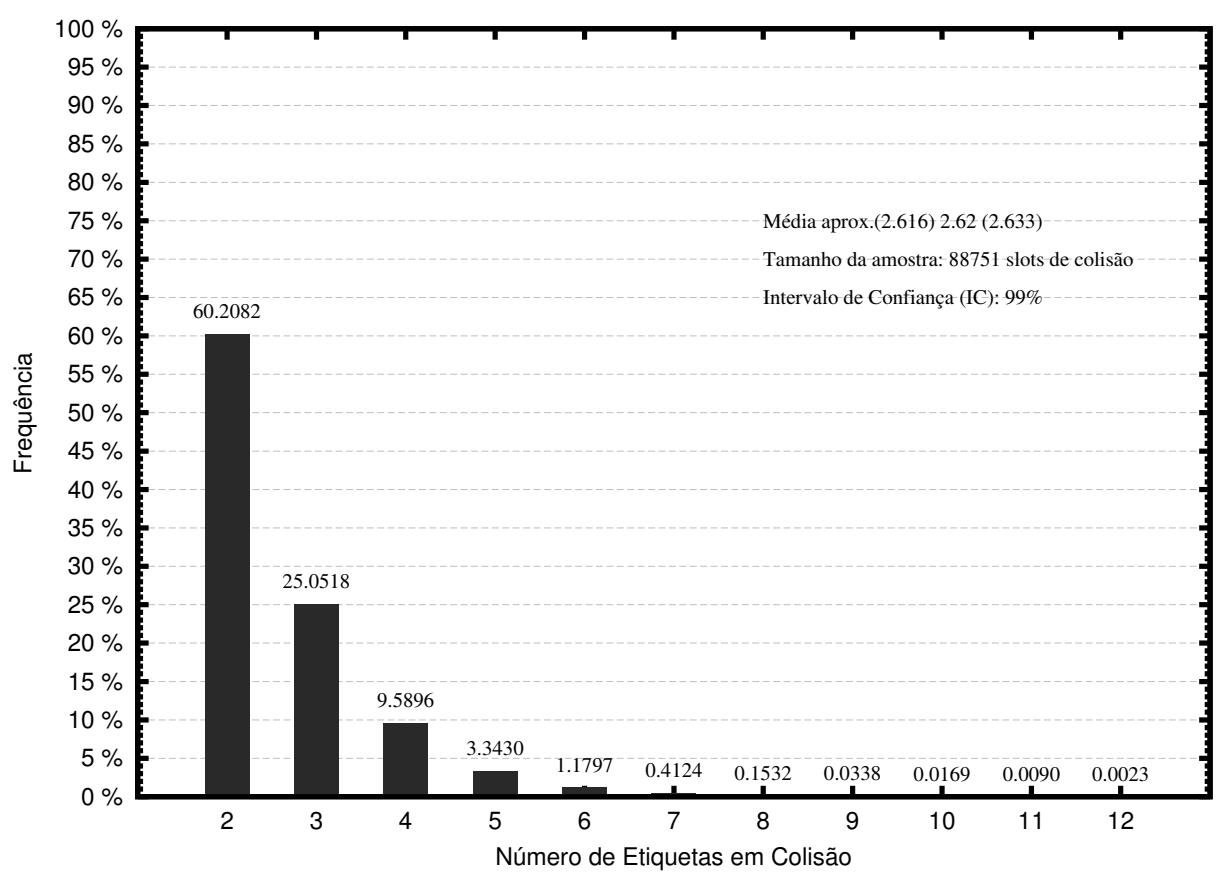

Figura 5.16: Média de Colisões por Slot

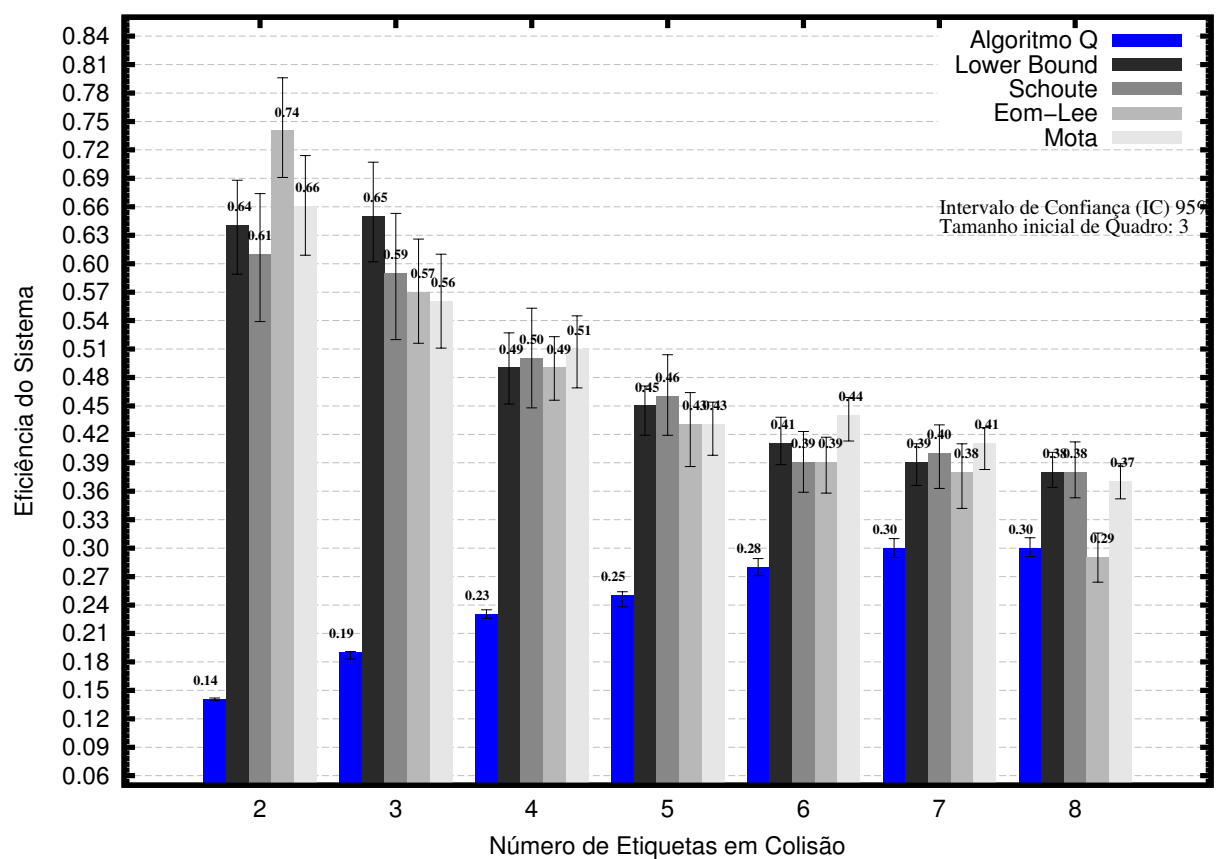

Figura 5.17: Resolução de colisões utilizando diferentes métodos

ao invés de 2.39. O valor 2.62 foi gerado a partir da média da quantidade de etiquetas em colisão por slot, conforme mostrado anteriormente na Figura 5.16.

Após os experimentos anteriores, chegou-se na definição completa do procedimento de resolução de colisões, de acordo com o Algoritmo 8.

Inicialmente, define-se o tamanho inicial do quadro com valor igual a 3 (inteiro mais 


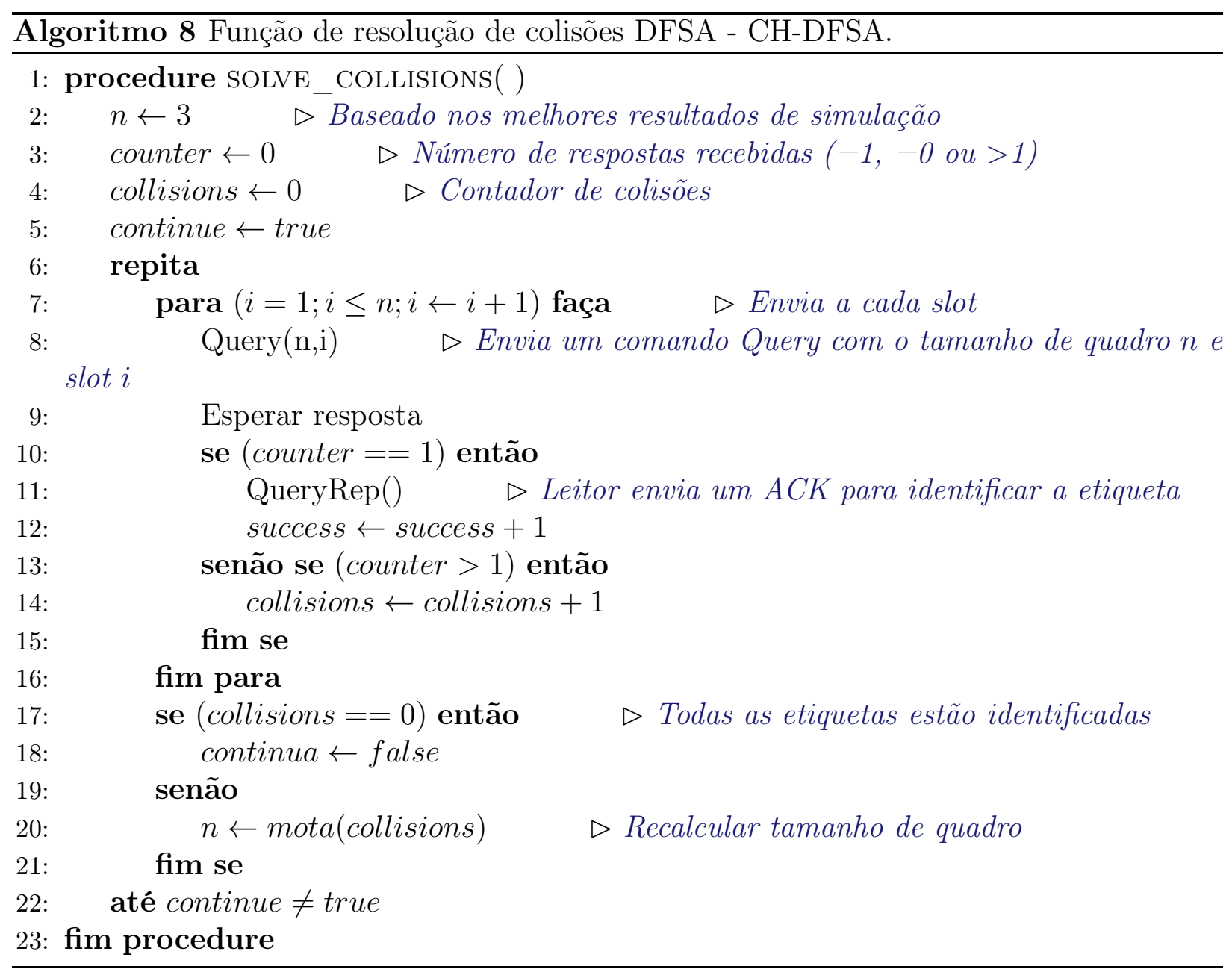

próximo da média 2.62) (linha 2). Posteriormente são definidos os contadores de respostas recebidas (linha 3) e de colisões (linha 4). O laço das linhas de 7-16 realiza o funcionamento padrão de um algoritmo DFSA, onde para cada slot é enviado um comando Query (linha 8) e espera-se as respostas (linha 9). Caso o número de respostas seja igual a 1 (linha 10), identifica-se a mesma com um comando QueryRep (linha 11). Caso mais de uma etiqueta responda (linha 13), incrementa-se o contador de colisões. Ao término do laço, temos o fim do primeiro quadro. Se nenhuma colisão ocorreu, então todas as etiquetas estão identificadas (linhas 17-18), e o algoritmo é encerrado (linhas 18 e 22). Caso contrário, calcula-se um novo tamanho de quadro a partir da quantidade de colisões, seguindo o algoritmo de Mota (linhas 19-20) e o laço das linhas 7-16 é reiniciado. O algoritmo termina quando não ocorrerem colisões dentro de um quadro. 


\subsubsection{Resultados}

Foram realizadas simulações para avaliação de desempenho dos algoritmos propostos, utilizando-se o nsRFIDsim apresentado na Seção 5.2. O objetivo dos experimentos foi a comparação de resultados publicados na literatura com os resultados obtidos pelos algoritmos propostos. Os parâmetros avaliados foram a eficiência do sistema $\left(S_{e f}\right)$ e a quantidade total de slots utilizados durante todo o processo de identificação, incluindo os slots gastos no procedimento inicial de estimativa do número de etiquetas. Os resultados foram obtidos após 20 simulações, sendo extraída a média e intervalo de confiança. Todos os experimentos foram realizados em um servidor equipado com processador Intel Core i7-2700K 3,5GHz, 16GB de RAM, 1TB de espaço em disco e sistema operacional Debian GNU/Linux versão 6.0 .

A Figura 5.18 apresenta a eficiência do sistema quando utilizados diferentes algoritmos anticolisão, como Schoute (Schoute, 1983) (com tamanho inicial de quadro em 128), (Eom e Lee, 2010) (com tamanho inicial de quadro em 128), Algoritmo Q (EPCglobal, GS1 Inc. , 2015) e o proposto nesta seção, o NEDFSA.

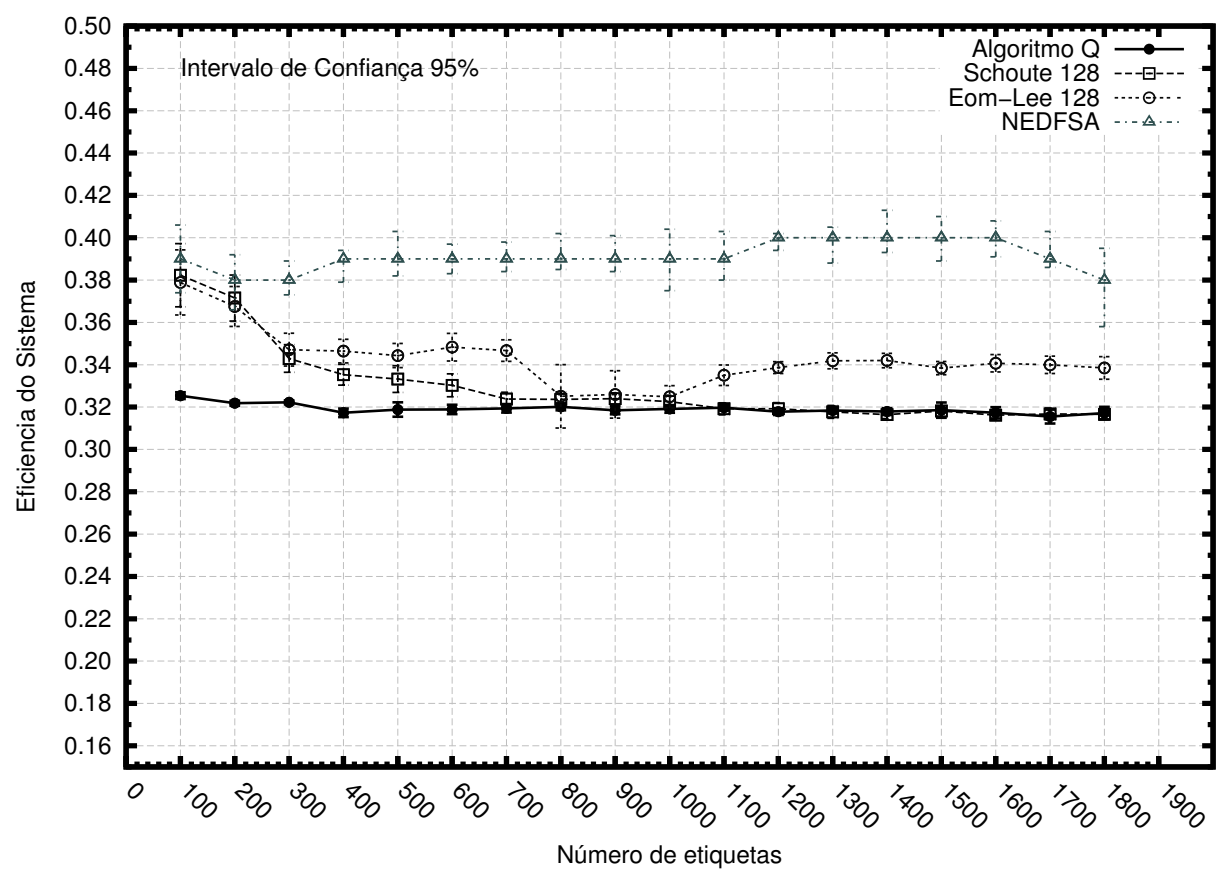

Figura 5.18: Eficiência do sistema

A quantidade de etiquetas nos experimentos variou de 100 a 1800 . Considerando os intervalos de confiança, o $S_{\text {ef }}$ médio do NEDFSA atingiu 39\%. Quando a quantidade de 
etiquetas é baixa, entre 100 e 200, o desempenho do NEDFSA é similar ao de Schoute, fato este justificado pelo tamanho inicial do quadro de 128 para Schoute, o que maximiza o desempenho do mesmo. Já quando a quantidade ultrapassa 300 etiquetas, o NEDFSA obtêm um ganho claramente superior a todos os demais.

A Figura 5.19 mostra a quantidade de slots utilizados durante todo o processo de identificação e reforça os resultados apresentados na Figura 5.18.

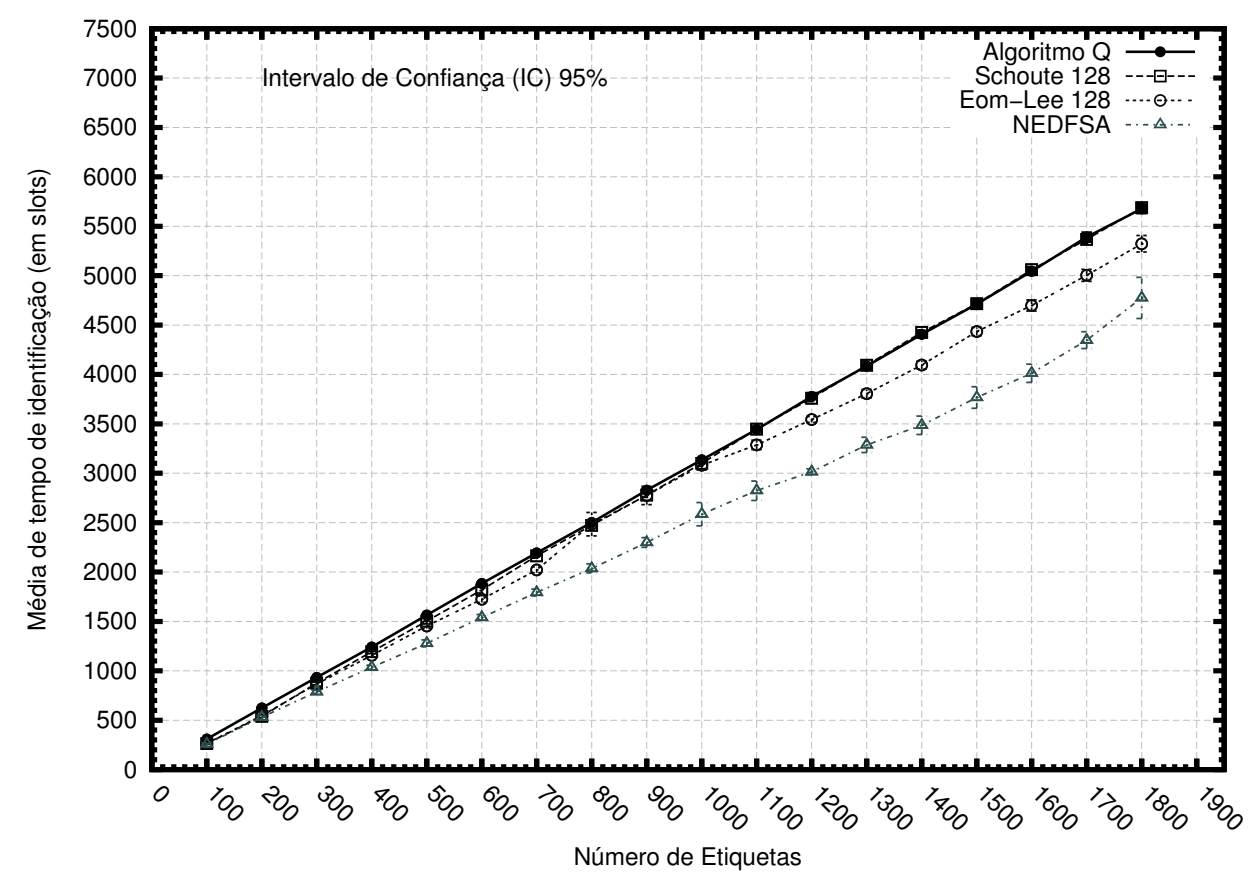

Figura 5.19: Total de slots

Todas as curvas apresentam-se próximas de uma função linear. Quanto menor o ângulo melhor o desempenho. Este gráfico também mostra claramente o melhor desempenho do NEDFSA.

Já a Figura 5.20 ilustra a redução percentual do número de slots utilizados de todas as propostas, comparadas ao Algoritmo Q. Resultados negativos significam redução na quantidade de slots e consequentemente melhoria de desempenho.

Observa-se que para quaisquer quantidades de etiquetas, o NEDFSA reduz a quantidade de slots necessários para a identificação de todas as etiquetas. Por exemplo, quando a quantidade de etiquetas é 800, temos redução do número de slots de aproximadamente $14 \%$ quando utilizado o NEDFSA, 1,12\% para Eom-Lee e 0,6\% para Schoute. O gráfico da Figura 5.19111 ilustra as vantagens do NEDFSA, pois para todos os valores do eixo $x$ o 


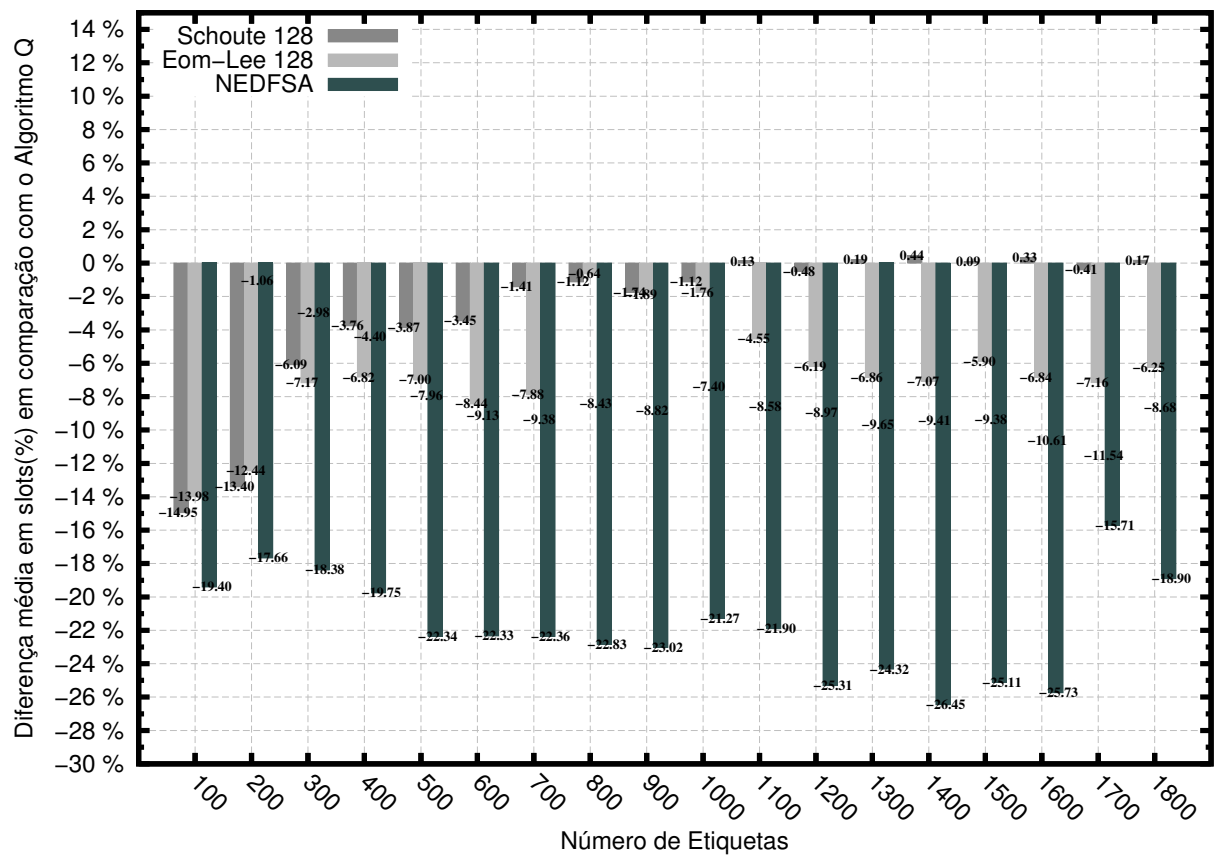

Figura 5.20: Redução de slots comparado ao Algoritmo $Q$

NEDFSA possui redução significativamente maior que os demais.

\subsubsection{Conclusões parciais}

O algoritmo proposto para o funcionamento do NEDFSA não apenas apresentou desempenho melhor que o Algoritmo Q, como também em relação às propostas de Schoute (1983) e Eom e Lee (2010). O mesmo também evita a complexidade adicionada pela proposta de Eom e Lee (2010). Além disso, uma vez que o processo de estimativa é realizado uma única vez, e com overhead baixo, somando-se ao fato da resolução imediata de colisões, o mesmo não é afetado pela quantidade de etiquetas. Quando a quantidade de objetos aumenta ou diminui significativamente, a eficiência não diminui.

\subsection{Benchmark para avaliação de desempenho de siste- mas RFID}

Conforme observado nos Capítulos 3 e 4, existem diversos mecanismos anticolisão propostos para RFID e existem diversas métricas que podem ser usadas para classificar quais desses mecanismos são os melhores para um dado cenário. A proposta de novos algoritmos 
sempre exige uma comparação com aqueles existentes. Embora o simulador nsRFIDSim seja útil para simular cenários realistas em que os nós se movimentam, em certas situações não há necessidade de tais simulações, bastando considerar que as etiquetas estejam ou não estejam no alcance dos leitores. Isso permite avaliar muitas das métricas apresentadas no Capítulo 4. Um simulador que permita facilmente a comparação de diversos algoritmos em relação a diversas métricas e que seja desenvolvido desde o início pensando em ser extensível, a fim de permitir a inclusão de novos algoritmo, portanto é algo ausente na literatura de mecanismos para sistemas RFID. Assim, esta seção apresenta o jRFIDSim, um simulador com escopo na camada de acesso ao meio, ou seja, no processo de identificação das etiquetas. O jRFIDSim foi desenvolvido com o objetivo de poder ser usado como benchmark para avaliação de desempenho de novos mecanismos para sistemas RFID. Ele já possui implementados o padrão EPCGlobal C1G2 (EPCglobal, GS1 Inc., 2015), os algoritmos de Schoute (Schoute, 1983), Eom-Lee (Eom e Lee, 2010), o NEDFSA (Seção 5.3), o Lower Bound (Vogt, 2002) e o DBTSA (Wu et al., 2013). Além dos mecanismos descritos anteriormente, implementouse também um mecanismo teórico denominado ODFSA, que representa uma situação em que o leitor conhece previamente a quantidade de etiquetas e sempre ajusta o tamanho dos quadros de acordo com a quantidade de etiquetas restantes. Apesar de não ser possível na prática, o ODFSA pode ser útil para fins de comparação. A Figura 5.21 ilustra a organização do jRFIDsim.

A suíte proposta está dividida em duas partes:uUm simulador escrito em JAVA e scripts de benchmark escritos em linguagem R. O simulador precisa ser executado primeiramente com o fim de gerar dados para serem analisados pelos scripts em R. O usuário precisa informar quais algoritmos devem ser avaliados como entrada e o simulador gera a contagem de slots (sucesso, colisão e vazio) como saída. Esses dados são convertidos em um conjunto de dados e o script em $\mathrm{R}$ calcula várias métricas relacionadas a energia, tempo e tráfego. Finalmente, a utilização das informações calculadas podem fornecer comparações gerais sobre os algoritmos selecionados como gráficos e tabelas.

A principal vantagem da utilização do jRFIDsim por pesquisadores da área é que apenas será necessário implementar o novo método no simulador, uma vez que todas as demais tarefas são realizadas pelo módulo de benchmark. Este fato também evita que vários métodos 


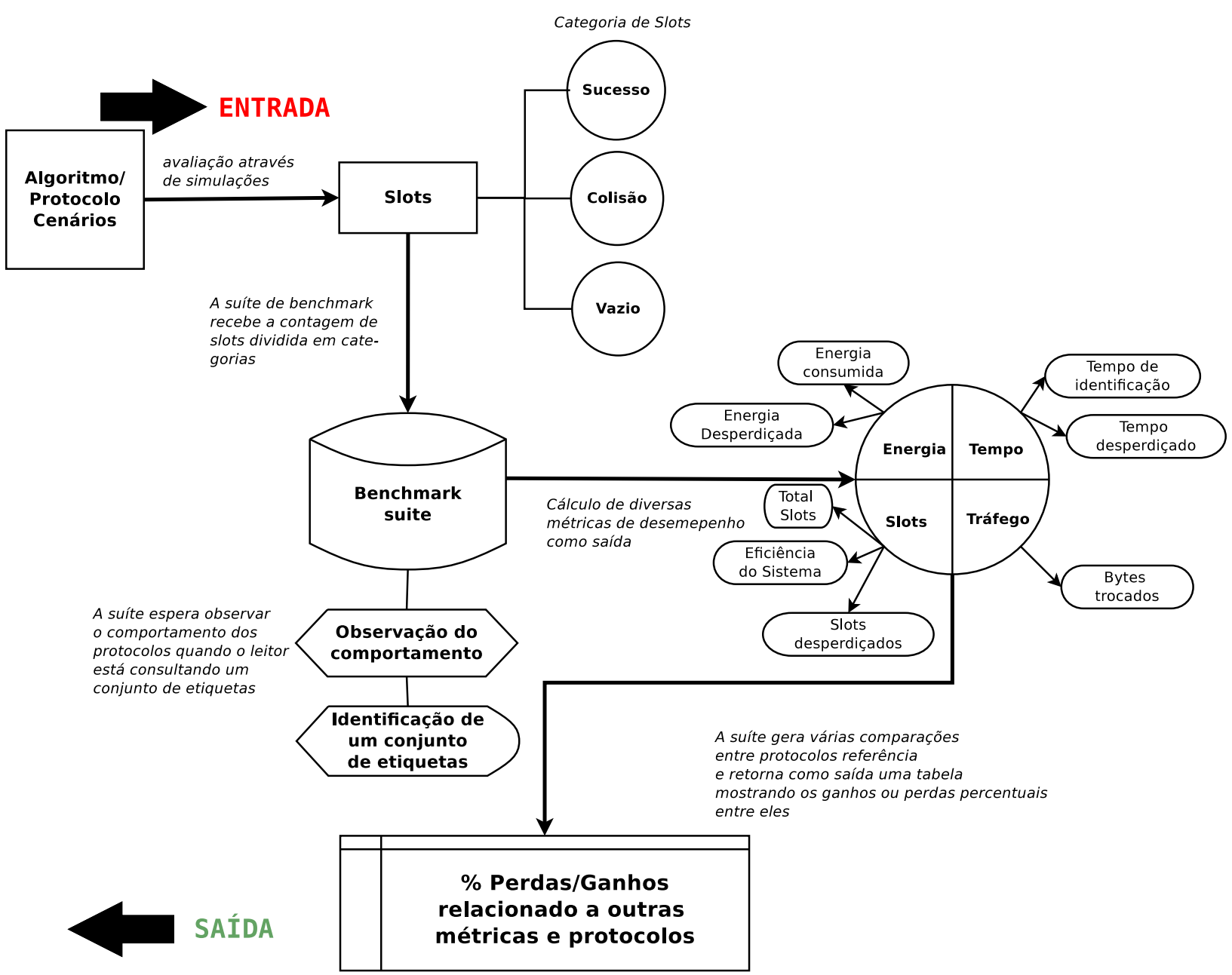

Figura 5.21: Organização do jRFIDsim.

da literatura precisem ser repetidamente implementados. O usuário pode também controlar diversos parâmetros como a quantidade inicial e final de etiquetas, número de repetições, intervalos de confiança e tamanhos iniciais de quadros, que são os principais parâmetros utilizados na literatura (Wu et al., 2013).

\subsubsection{Visão geral do jRFIDsim}

O jRFIDsim, ilustrado na Figura 5.22, modela a camada de acesso ao meio de sistemas RFID passivos baseado em várias propostas da literatura, permitindo a simulação de cenários com diferentes quantidades de etiquetas.

Como o objetivo é a avaliação da eficiência dos algoritmos anticolisão, o simulador não é baseado em eventos discretos. Ao invés disso, apenas a quantidade de etiquetas pode ser variável, permitindo assim que a simulação seja realizada de forma mais rápida e eficiente 


\begin{tabular}{|l|}
\multicolumn{1}{|c|}{ Simulator } \\
\hline \#tags: ArrayList<Tag> \\
\#slot: ArrayList<Slot> \\
\#idl: int \\
\#col: int \\
\#suc: int \\
\hline \#initData()( ) : void \\
\#initTagList(): void \\
\#initCurrentFrame(): void \\
\#sendQuery(): void \\
\#prepareSlots(): void \\
\#identifyTags(): void \\
\#identifyTagsInCollision(): void \\
\#finalizeFrame(): void \\
\#standardDFSA(): void \\
\#startDFSA(): void
\end{tabular}

\begin{tabular}{|l|}
\hline \multicolumn{1}{|c|}{ Tag } \\
\hline \#code: int \\
\#rng16: int \\
\#state: int \\
\hline +setRng16(up:int): void \\
+updateRng16(value: int): void \\
+getRn16(): int \\
\hline
\end{tabular}

\begin{tabular}{|c|}
\hline Slot \\
\hline -tags: ArrayList<Tag $>$ \\
\hline +addTag(code: Tag) : void \\
\hline
\end{tabular}

Figura 5.22: Diagrama de classes do jRFIDsim.

para este objetivo específico, tornando-a mais rápida do que se a mesma fosse realizada com o nsRFIDSim por exemplo. No final da simulação são gerados traces de resultados que poderão ser utilizados para a análise de diversas métricas de desempenho sobre cada um dos métodos simulados, como por exemplo a vazão do método, tempo de identificação e consumo de energia.

\section{A Classe Simulator}

A classe Simulator modela as características de um sistema RFID, composto por um leitor e um conjunto de etiquetas (atributo tags). O leitor por sua vez possui um conjunto de quadros, cada quadro sendo formado por um conjunto de slots (atributo slot). Além disso, o leitor implementa contadores para cada tipo de slot gerado (atributos col,idl e suc). Como o foco do simulador é a avaliação de mecanismos anticolisão baseados no padrão DFSA, o método standardDFSA implementa genericamente este funcionamento, independente do algoritmo específico a ser simulado. A Figura 5.23 descreve o método standardDF SA. O laço das linhas 2 a 10 representa as operações realizadas em um quadro específico do processo de identificação e é repetido enquanto houverem slots de colisão. Na linha 3 a operação de preparação do quadro é realizada, como a disponibilização dos slots que poderão ser escolhidos pelas etiquetas. Já o comando sendQuery simula o comando Query dos leitores 
(linha 4), operação que informa os possíveis slots que poderão ser escolhidos pelas etiquetas. Neste momento cada uma das etiquetas seleciona aleatoriamente um slot para responder. Na linha 6, o comando prepareSlots aloca as etiquetas a cada um dos slots disponíveis. A partir deste ponto, o leitor inicia a chamada de cada um dos slots, conforme o comando identifyTags da linha 7. Para cada slot é computado se o mesmo é do tipo sucesso (etiqueta identificada), colisão (adiciona ao contador de colisões) ou vazio (adiciona ao contador de vazios). Finalmente o método finalizeFrame (linha 8) é chamado para verificar se é o momento de definir um novo quadro ou de finalizar o processo.

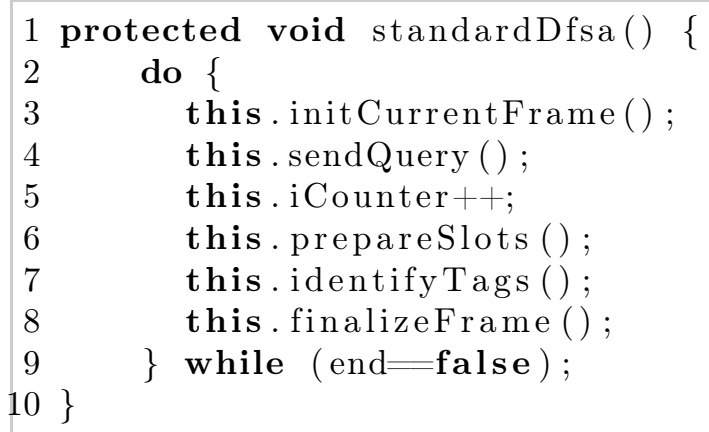

Figura 5.23: Método DFSA genérico.

Algoritmos baseados no padrão PDFSA-b podem ser facilmente adicionados através da modificação do método finalizeFrame, que é o momento da definição do próximo quadro. Para algoritmos que não baseiam-se no funcionamento do PDFSA-b, devem ser adicionados novos métodos específicos, porém com o aproveitamento dos demais métodos já implementados.

Finalmente, a Figura 5.24 apresenta o método que realiza a simulação como um todo do algoritmo desejado. Baseado nos parâmetros definidos pelo usuário no momento da execução da simulação, o método realiza o processo de identificação para vários conjuntos de etiquetas, com a possibilidade de várias repetições para chegar-se a uma média (laço da linha 3). O condicional das linhas 6 a 8 chama o método anteriormente apresentado standardDFSA para o caso de algoritmos baseados em PDFSA-b. Caso contrário é definido uma condição para cada novo algoritmo(linhas 9 a 29). As linhas 35 a 40 realizam as operações estatísticas para cada tipo de slots observados, com o objetivo de gerar no final a média com os respectivos intervalos de confiança. Dessa forma, para a adição de novos algoritmos a serem simulados, deve-se também modificar o método startDFSA para inclusão do mesmo dentro 
do laço principal do simulador.

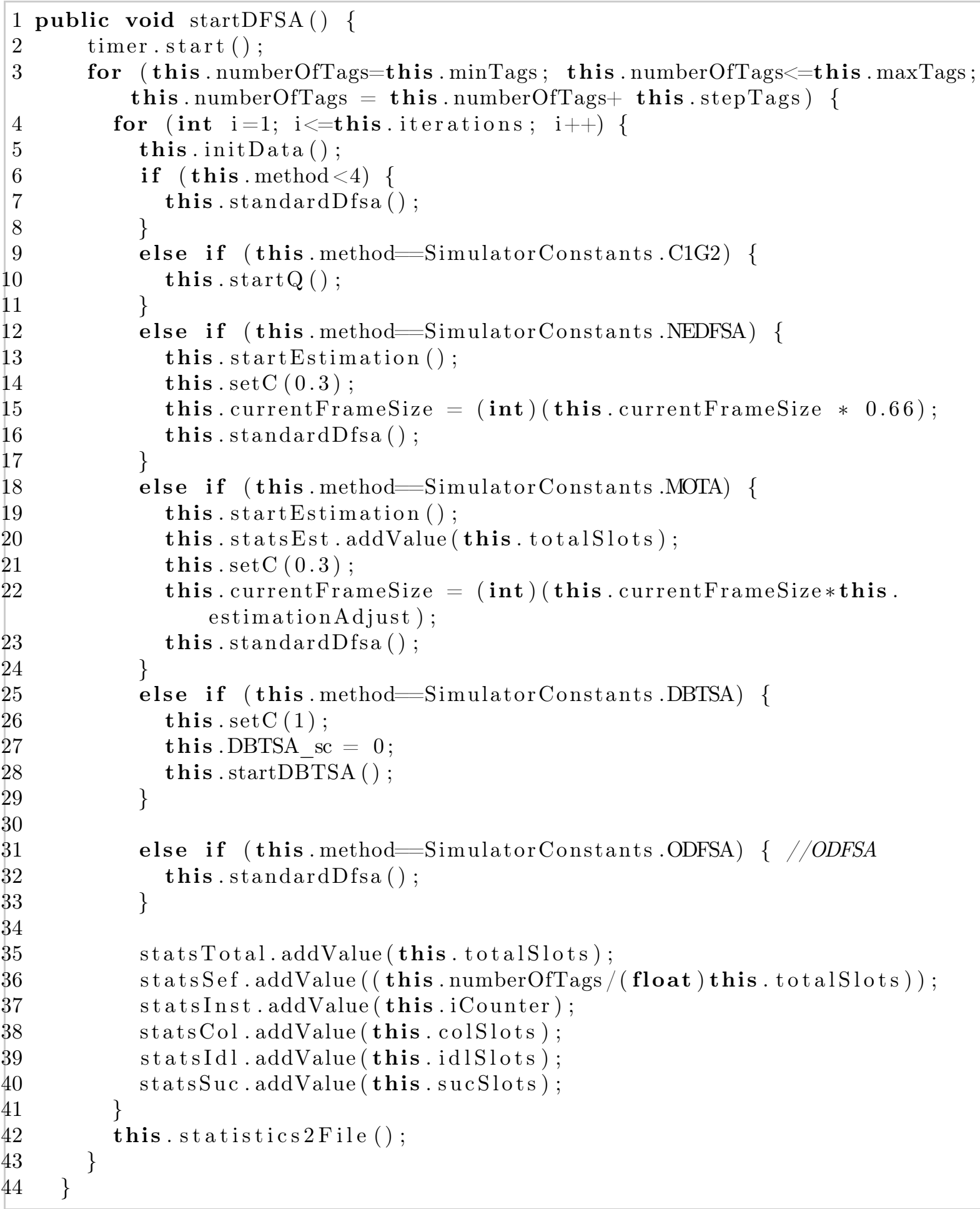

Figura 5.24: Método de simulação geral.

\section{As Classes Tag e Slot}

A classe Tag, que representa uma etiqueta possui em sua estrutura interna os elementos essenciais de uma etiqueta real, como seu código identificador (code), seu slot aleatório 
(rng16) e seu estado (state). Já a classe Slot implementa um vetor que guarda o conjunto de etiquetas que o selecionaram para responder e um método que adiciona uma etiqueta a si mesmo.

\subsubsection{Desempenho do jRFIDsim}

A Figura 5.25 ilustra os tempos de simulação para cada um dos algoritmos implementados no jRFIDsim. Foram considerados parâmetros típicos adotados na literatura para avaliação de propostas anticolisão para sistemas RFID. Estes parâmetros típicos envolvem uma quantidade de etiquetas variando de 100 a 1000 com intervalos de 100, intervalo de confiança de $95 \%$, e mil repetições para cada algoritmo.

O tempo médio para este tipo de simulação fica em torno de um minuto, exceto para o algoritmo Q que por tratar-se de um método que gera novos quadros a cada nova rodada de identificação, tem seu tempo de simulação bem mais elevado devido ao custo desta criação contínua de novos quadros. Quando comparado a simuladores de eventos discretos como o nsRFIDsim (Seção 5.2) e o RFIDsim (Floerkemeier e Sarma, 2009) este tempo pode chegar a ser quase vinte vezes mais rápido, pois os mesmos não foram desenvolvidos para avaliar apenas o ponto específico do mecanismo de acesso ao meio, permitindo a simulação também de outras variáveis como mobilidade e integração com outras tecnologias.

\subsubsection{Validação do jRFIDsim}

A avaliação de confiabilidade do jRFIDsim foi realizada através da simulação computacional, com o objetivo de comparar-se os resultados experimentais com os analíticos obtidos por Eom e Lee (2010); Namboodiri et al. (2012); Schoute (1983); Wu et al. (2013). Cada simulação foi realizada individualmente em 1000 repetições, e as médias dos resultados das 1000 repetições foram calculadas. A Figura 5.26 mostra que as linhas dos modelos experimental e analítico estão quase que sobrepostas, garantindo a confiabilidade do simulador (Namboodiri et al., 2012).

Na Figura 5.27 compara-se o número total de slots utilizados na identificação de etiquetas utilizando o mecanismos DBTSA. Pode- se observar que, assim como na análise da 


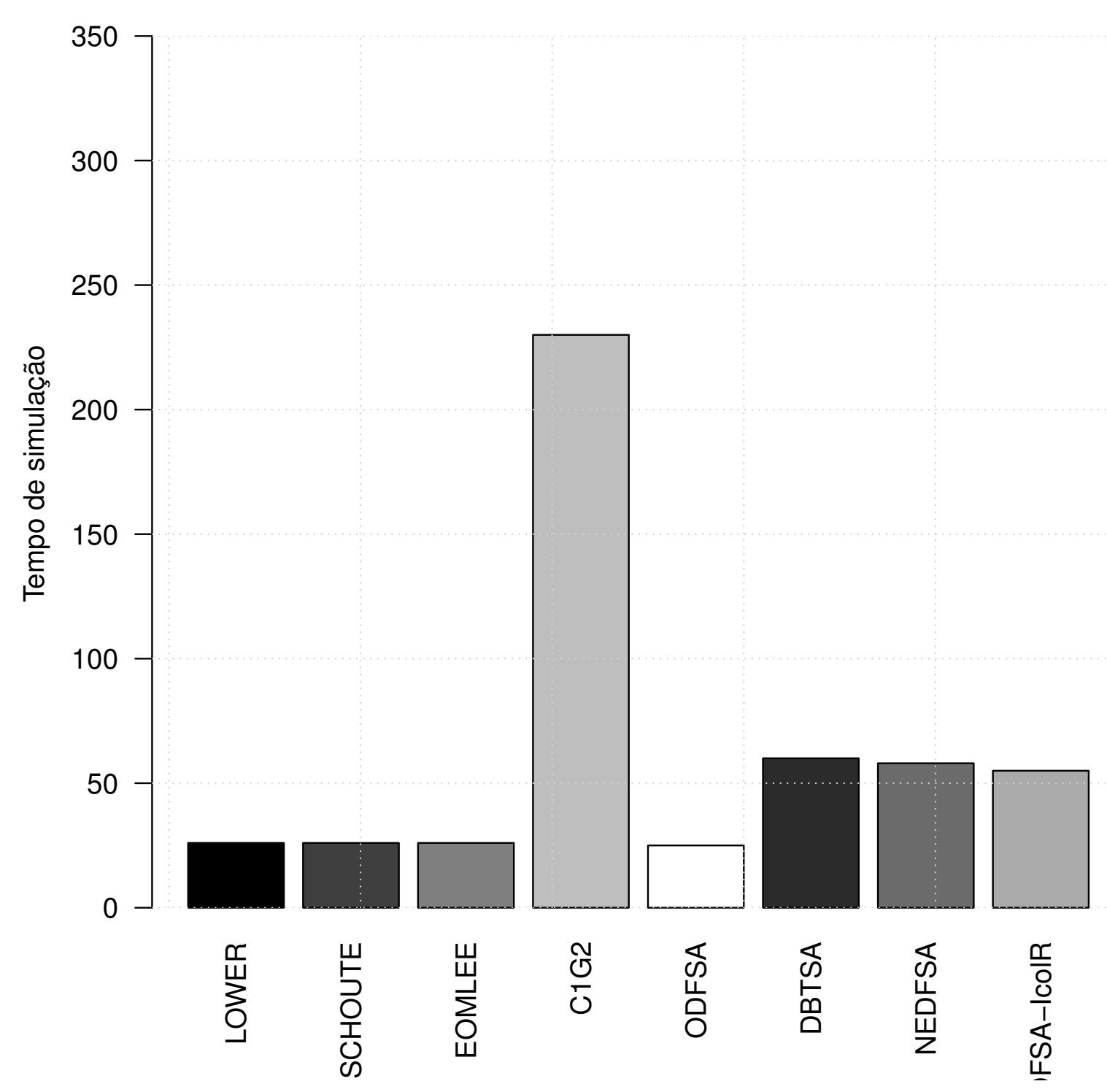

Figura 5.25: Desempenho do jRFIDsim.

eficiência do sistema para o Algoritmo Q, os resultados das simulações estão de acordo com os resultados analíticos.

As Figuras 5.28 e 5.29 fornecem a métrica $S_{e f}$ quando a quantidade de etiquetas varia de 100 até 5200 para os algoritmos Lower Bound (Vogt, 2002) e Schoute (Schoute, 1983), respectivamente. Assim como os resultados mostrados nas Figuras 5.26 e 5.27, as curvas dos modelos analítico e experimental estão quase que sobrepostas.

Os resultados apresentados validam a confiabilidade do simulador uma vez que o comportamento do mesmo está dentro do esperado (Floerkemeier e Sarma, 2009). 


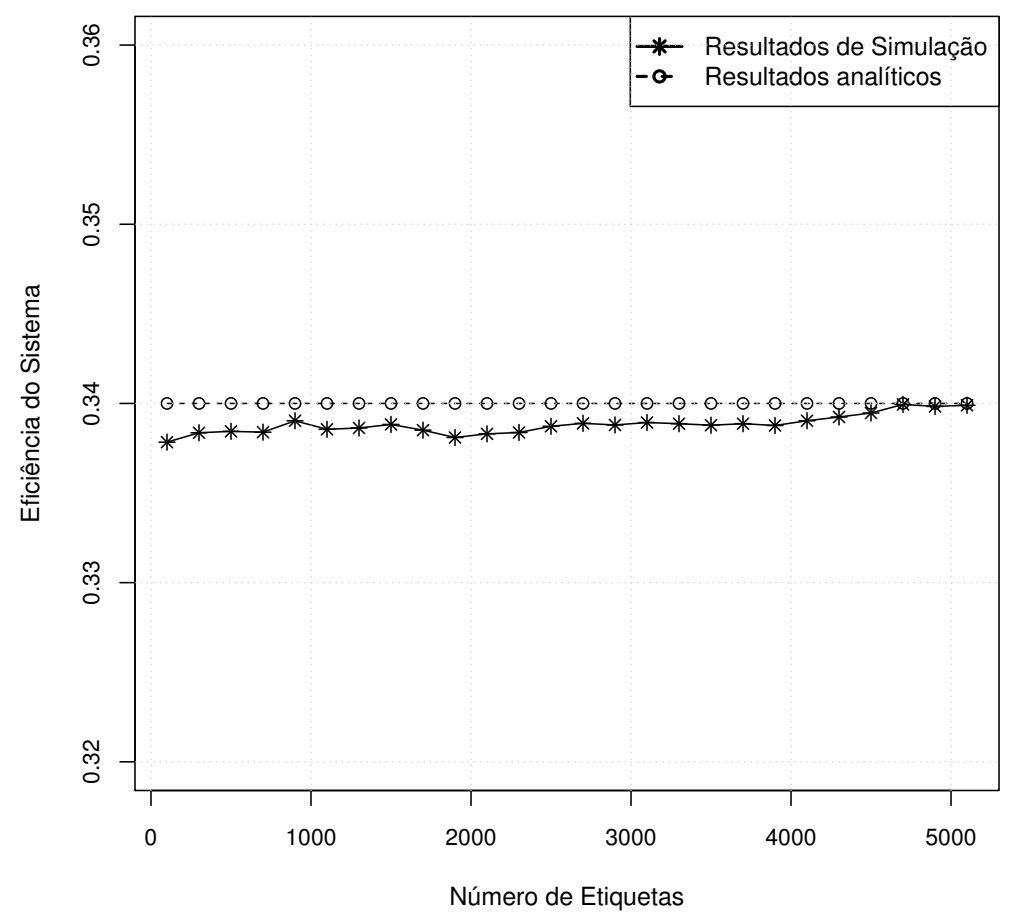

Figura 5.26: Eficiência do Sistema (C1G2).

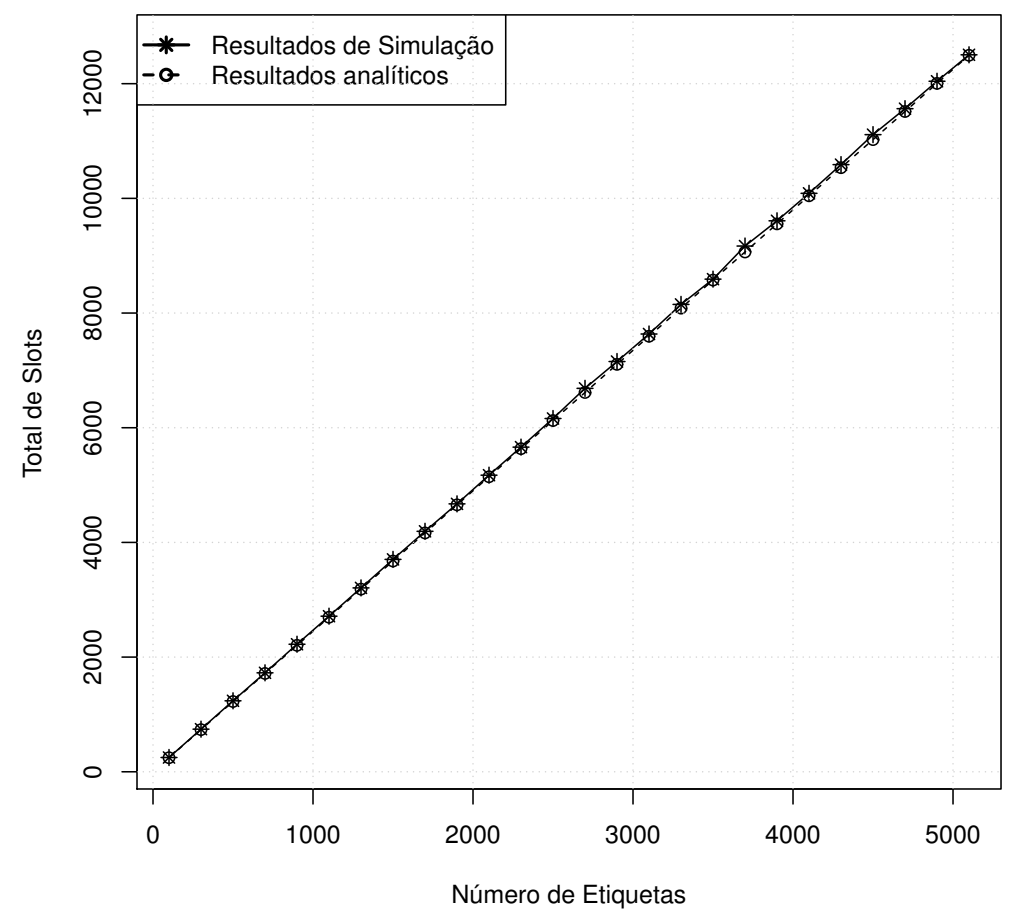

Figura 5.27: Número total de slots utilizados (DBTSA). 


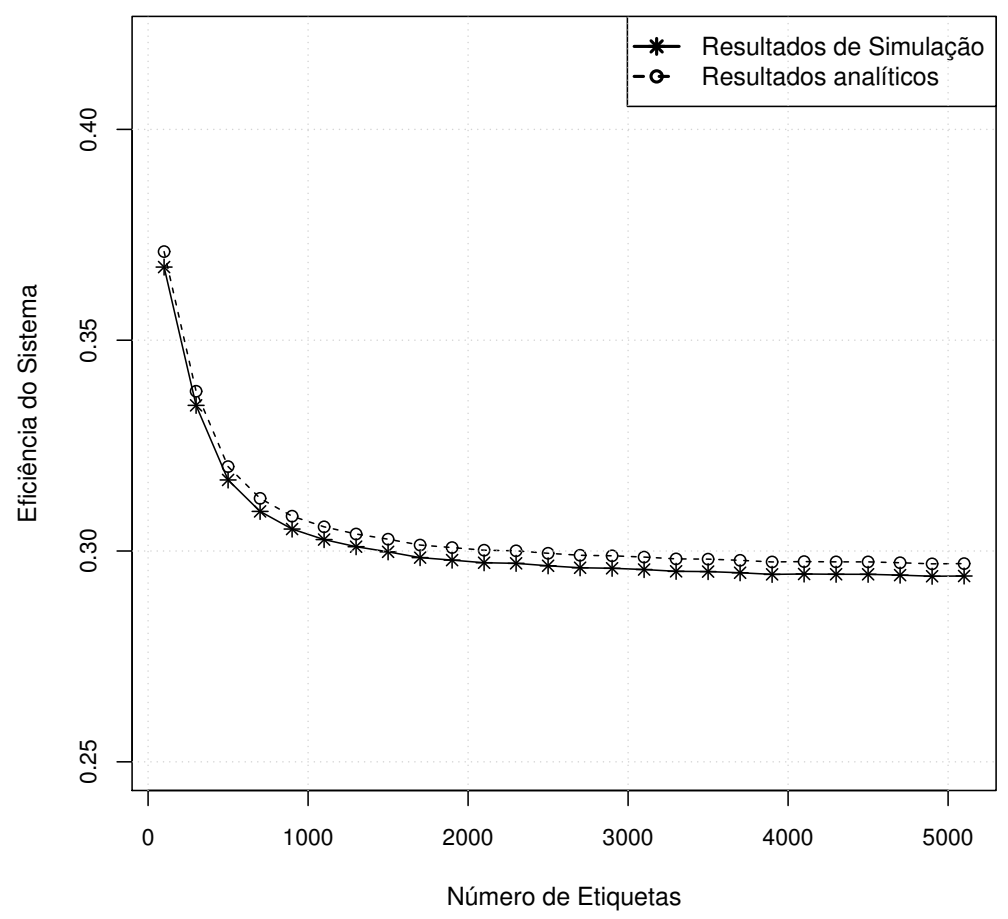

Figura 5.28: Eficiência do Sistema (Lower Bound).

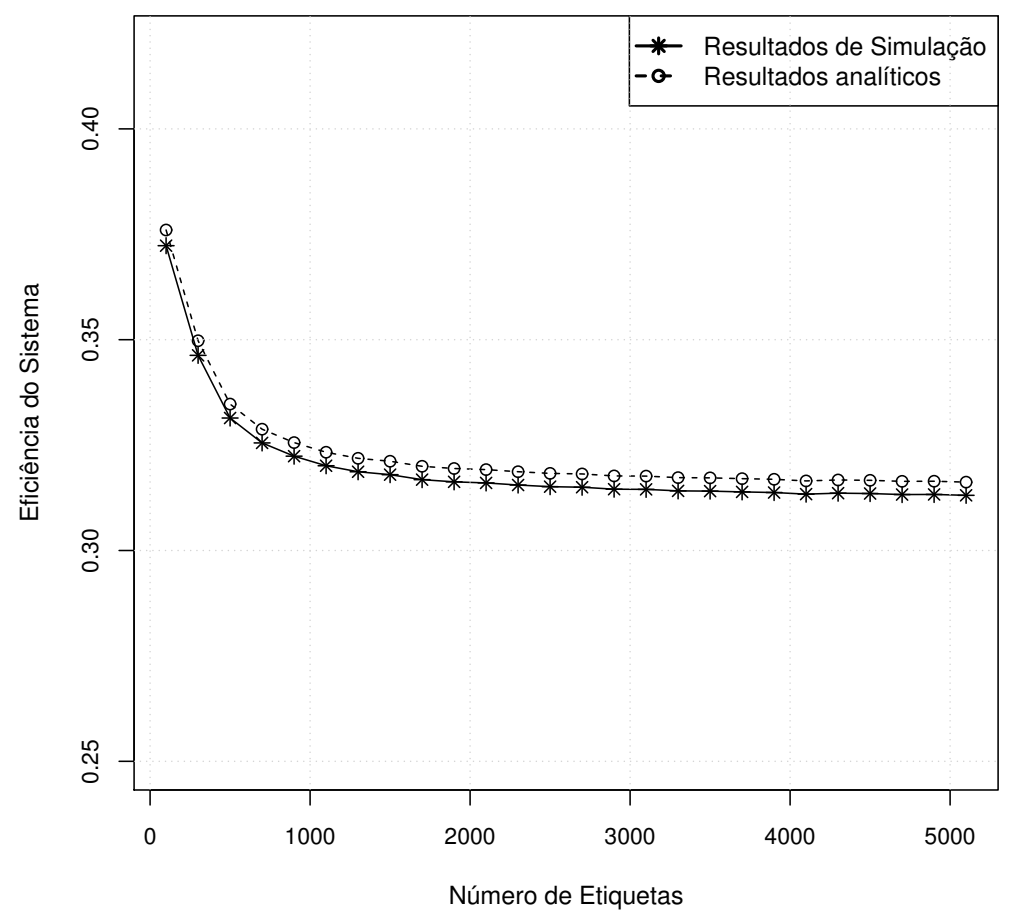

Figura 5.29: Eficiência do Sistema (Schoute). 


\subsubsection{Os testes de benchmark do jRFIDsim}

Os testes de benchmark visam comparar os algoritmos implementados no jRFIDsim em relação às principais métricas adotadas na literatura, como eficiência do sistema $\left(S_{e f}\right)$, eficiência de uso do canal $\left(C_{e f}\right)$, tempo de identificação, energia consumida e número total de slots. Para cada métrica é gerada uma tabela resumo com o valor correspondente da métrica para determinada quantidade de etiquetas (linhas) e algoritmo (colunas). Cada tabela gerada encontra-se em formato para fácil geração de gráfico comparativo, conforme amplamente utilizado na literatura. A Tabela 5.6 ilustra a saída do jRFIDsim com relação a métrica eficiência do sistema. As linhas representam a quantidade de etiquetas e as colunas os métodos simulados. Por exemplo, o método DBTSA possui eficiência do sistema de 0.40 quando a temos a quantidade de etiquetas em 1500. O mesmo formato de tabela é gerado para as métricas tempo (total e desperdiçado), energia (total e desperdiçado), eficiência de uso do canal e quantidade de slots utilizados (total e desperdiçados), o que permite uma comparação abrangente entre os métodos implementados, o que pode aumentar a confiabilidade das comparações entre as propostas da literatura.

\subsubsection{Conclusões parciais}

Esta seção apresentou um software que almeja ser uma ferramenta de benchmark para avaliação de mecanismos anti-colisão para sistemas RFID passivos. O jRFIDSim está disponível sob licença General Public License (GPL) e pode ser obtido no sítio eletrônico em https://bitbucket.org/rafaelperazzo/jrfidsim. O jRFIDSim também é apresentado no trabalho científico (Mota, 2015a). 
Tabela 5.6: Exemplo de tabela da métrica Eficiência do Sistema.

\begin{tabular}{|c|c|c|c|c|c|c|c|c|}
\hline Qtde & Lower & Schoute & Eom-Lee & C1G2 & ODFSA & DBTSA & NEDFSA & IDFSA-IcolR \\
\hline 100 & 0.367 & 0.372 & 0.371 & 0.338 & 0.378 & 0.406 & 0.382 & 0.396 \\
300 & 0.335 & 0.346 & 0.349 & 0.338 & 0.371 & 0.407 & 0.393 & 0.406 \\
500 & 0.317 & 0.331 & 0.344 & 0.338 & 0.370 & 0.406 & 0.393 & 0.408 \\
700 & 0.309 & 0.326 & 0.345 & 0.338 & 0.370 & 0.408 & 0.397 & 0.409 \\
900 & 0.305 & 0.322 & 0.342 & 0.339 & 0.369 & 0.407 & 0.395 & 0.409 \\
1100 & 0.303 & 0.320 & 0.347 & 0.339 & 0.369 & 0.408 & 0.397 & 0.411 \\
1300 & 0.301 & 0.319 & 0.354 & 0.339 & 0.369 & 0.408 & 0.398 & 0.411 \\
1500 & 0.300 & 0.318 & 0.357 & 0.339 & 0.369 & 0.407 & 0.398 & 0.412 \\
1700 & 0.298 & 0.317 & 0.357 & 0.339 & 0.369 & 0.407 & 0.398 & 0.412 \\
1900 & 0.298 & 0.316 & 0.357 & 0.338 & 0.368 & 0.408 & 0.397 & 0.411 \\
2100 & 0.297 & 0.316 & 0.355 & 0.338 & 0.369 & 0.408 & 0.400 & 0.412 \\
2300 & 0.297 & 0.316 & 0.353 & 0.338 & 0.368 & 0.409 & 0.398 & 0.411 \\
2500 & 0.296 & 0.315 & 0.351 & 0.339 & 0.369 & 0.408 & 0.399 & 0.411 \\
2700 & 0.296 & 0.315 & 0.349 & 0.339 & 0.368 & 0.407 & 0.399 & 0.413 \\
2900 & 0.296 & 0.315 & 0.347 & 0.339 & 0.369 & 0.408 & 0.398 & 0.412 \\
3100 & 0.296 & 0.315 & 0.346 & 0.339 & 0.368 & 0.408 & 0.399 & 0.413 \\
3300 & 0.295 & 0.314 & 0.344 & 0.339 & 0.368 & 0.407 & 0.399 & 0.413 \\
3500 & 0.295 & 0.314 & 0.343 & 0.339 & 0.368 & 0.409 & 0.399 & 0.413 \\
3700 & 0.295 & 0.314 & 0.342 & 0.339 & 0.368 & 0.406 & 0.399 & 0.412 \\
3900 & 0.294 & 0.314 & 0.341 & 0.339 & 0.368 & 0.408 & 0.399 & 0.412 \\
4100 & 0.295 & 0.313 & 0.341 & 0.339 & 0.368 & 0.408 & 0.398 & 0.412 \\
4300 & 0.294 & 0.314 & 0.340 & 0.339 & 0.368 & 0.408 & 0.399 & 0.412 \\
4500 & 0.294 & 0.314 & 0.340 & 0.339 & 0.369 & 0.407 & 0.399 & 0.412 \\
4700 & 0.294 & 0.313 & 0.340 & 0.340 & 0.368 & 0.408 & 0.400 & 0.413 \\
4900 & 0.294 & 0.313 & 0.340 & 0.340 & 0.368 & 0.409 & 0.399 & 0.413 \\
5100 & 0.294 & 0.313 & 0.339 & 0.340 & 0.368 & 0.410 & 0.399 & 0.413 \\
\hline
\end{tabular}

\subsection{Um algoritmo eficiente em energia e tempo para RFID}

Em algoritmos DFSA, duas etiquetas que não colidem em determinado quadro podem vir a colidir em quadros subsequentes ao selecionarem um novo slot para responder em quadros posteriores. Se as colisões fossem tratadas imediatamente enquanto os demais slots "esperassem", essa situação seria evitada, o que é bom para o sistema pois reduz a quantidade de colisões desnecessárias.

Nesta Seção é apresentado o IDFSA-IColR (Improved DFSA with Instant Collision Resolution), um algoritmo anti-colisão para sistemas RFID passivos que trata a colisão no momento em que a mesma é detectada. Como consequência, o algoritmo mostra-se eficiente quando a métrica avaliada é o tempo necessário para identificar as etiquetas e, também, em termos de consumo de energia. A Subseção 5.5.4 apresenta os resultados que comprovam a 
eficiência deste algoritmo.

O IDFSA-IColR é formado por duas fases. A fase de estimativa e a fase DFSA principal. O algoritmo de estimativa, primeira fase do IDFSA-IColR, apresentado na Figura 5.30 funciona como a seguir: o leitor consulta as etiquetas 5 vezes. Se o leitor detecta pelo menos 3 slots de colisão (provavelmente o valor de Q é pequeno), então o valor de $Q$ é incrementado em uma unidade e o procedimento é reiniciado.

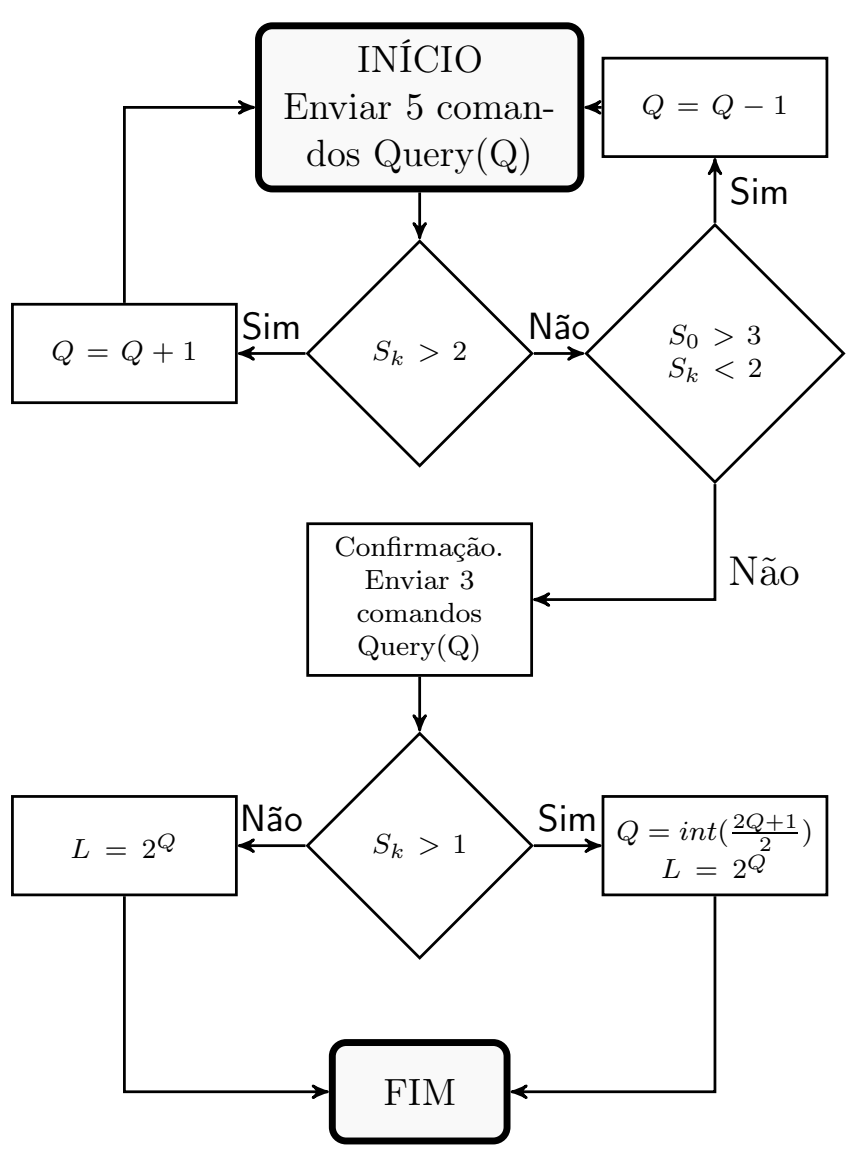

Figura 5.30: IDFSA-IcolR: Fluxograma.

Caso contrário, se o leitor recebe pelo menos 4 slots vazios e menos que 2 colisões (provavelmente o valor de Q é grande), então o valor de Q é decrementado em uma unidade e o procedimento é também reiniciado. Caso contrário o algoritmo entra na fase de confirmação. O leitor então consulta as etiquetas 3 vezes. Se o número de slots de colisão for maior do que 1 (provavelmente um ponto médio) então o procedimento retorna o valor médio entre $Q$ e $Q+1$. Caso contrátio o atual valor de $\mathrm{Q}$ é retornado, pois provavelmente este valor é o mais próximo da quantidade real de etiquetas.

O algoritmo completo do IDFSA-IcolR é mostrado no Algoritmo 9. 


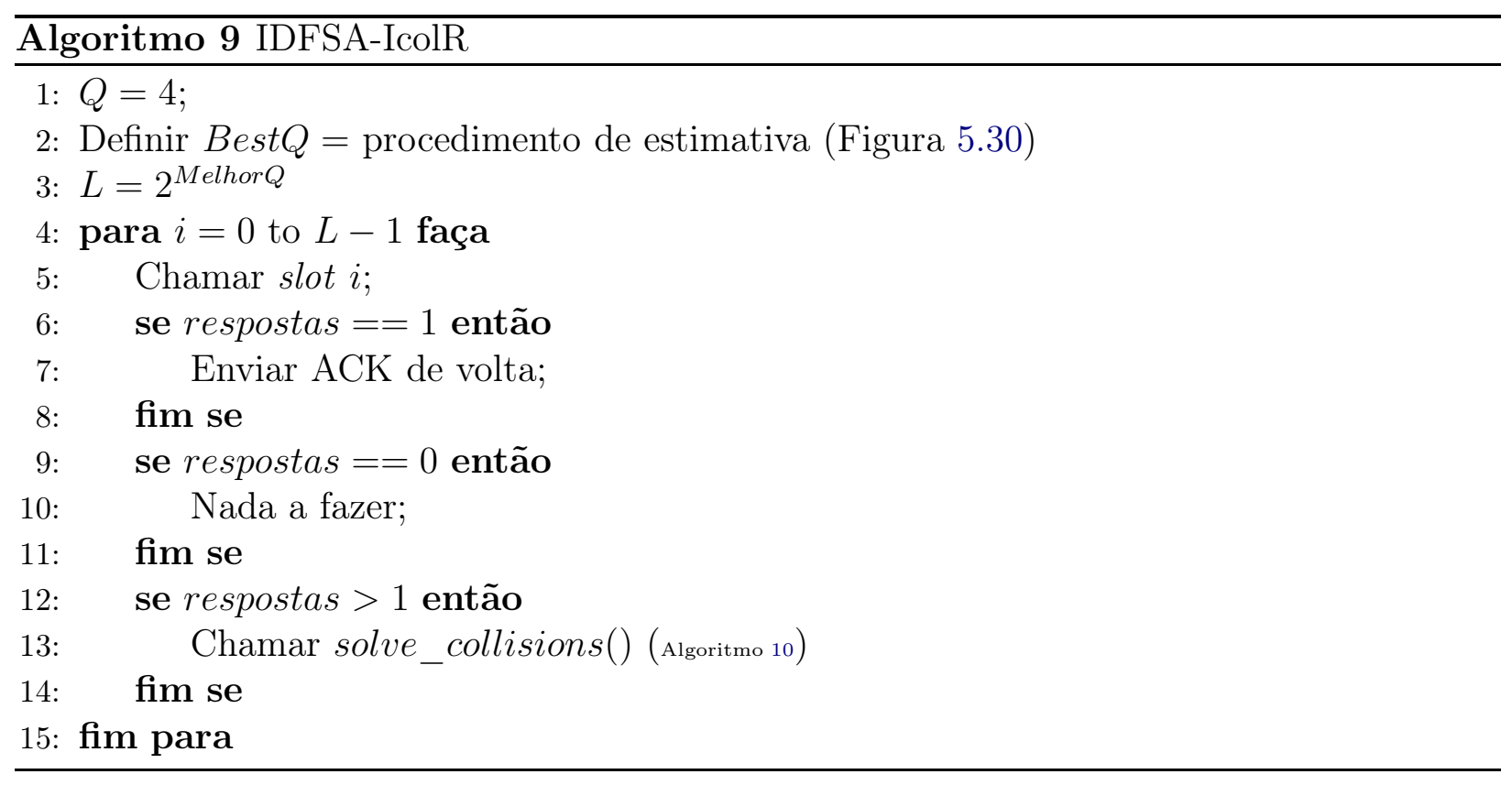

Inicialmente, a estimativa do número de etiquetas é realizada de acordo com o fluxograma da Figura 5.30. Como este passo ajusta o tamanho inicial do quadro para um valor razoável em relação ao número de etiquetas, a quantidade de colisões e colisões por slot são minimizadas. Posteriormente o algoritmo proposto inicia o procedimento principal DFSA (linhas 4-5). Quando um slot de colisão é encontrado (linha 12), este é tratado através do procedimento solve_collisions() (linha 13) (Algoritmo 10). Os slots de colisão são tratados pelo método de Schoute (Schoute, 1983) com tamanho inicial de quadro definido como 4 (Algoritmo 10 - linha 2). O algoritmo continua chamando os slots subsequentes até o final do quadro. Quando uma nova colisão é detectada, o procedimento solve_collisions() é novamente chamado.

O procedimento solve_collisions, descrito no Algoritmo 10, inicia definindo o tamanho inicial de quadro como 4 (linha 2).

Posteriormente o mesmo inicia o contador de respostas e colisões (linhas 3 e 4). As etiquetas em colisão do momento devem selecionar um número aleatório entre 0 e 3 (linha 6). Então o leitor chama os novos slots observando os tipos de respostas (linhas 8-16). Se nenhuma colisão é detectada em um quadro, o procedimento termina. Caso contrário, um novo quadro deve ser criado. Se mais de um slot de colisão for detectado, então o procedimento utiliza o método de Schoute (Schoute, 1983) para selecionar o tamanho do próximo quadro (linha 21). Se apenas uma colisão for observada, então o próximo quadro é 


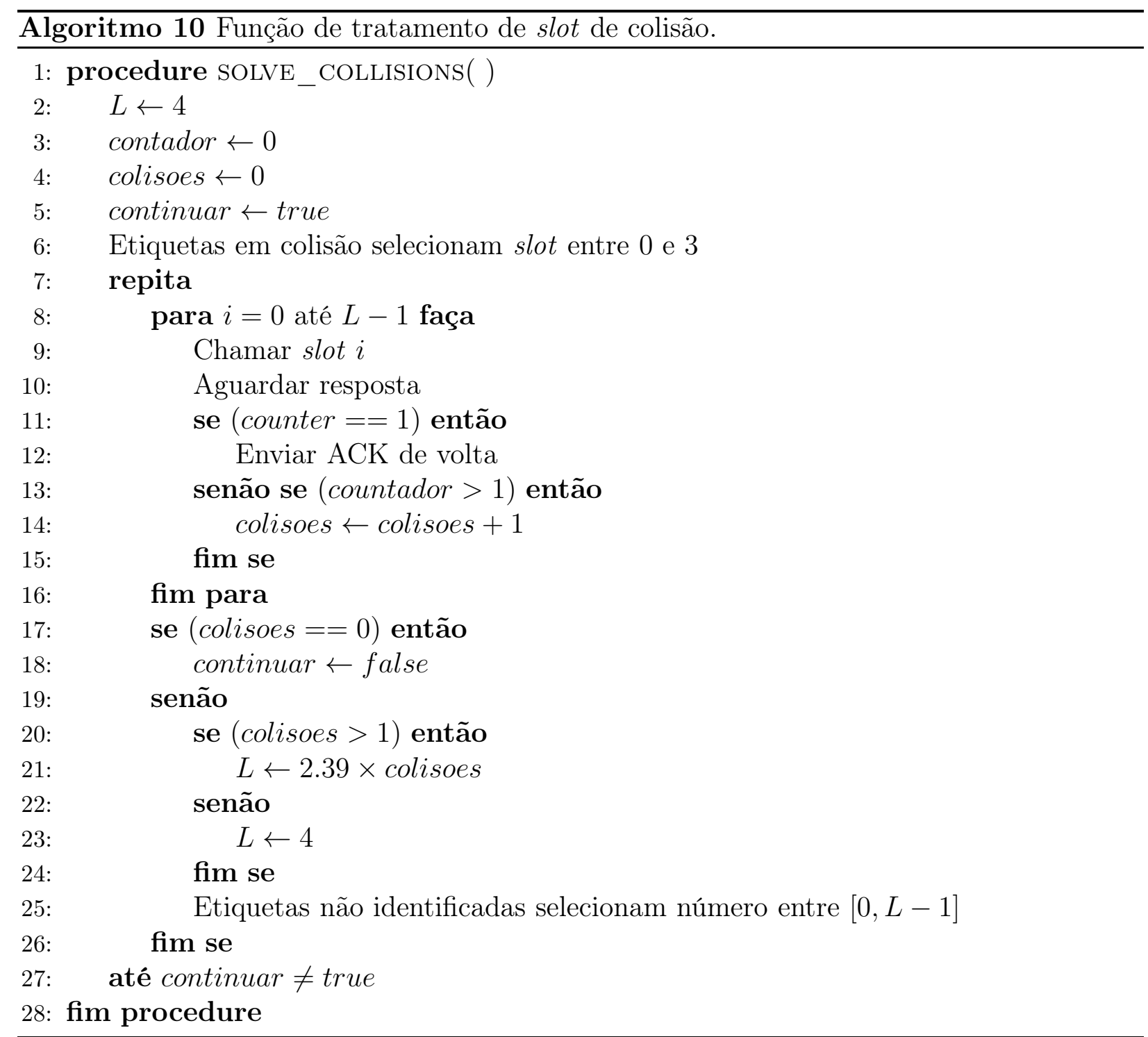

novamente definido como 4 (linha 23). As etiquetas em colisão restantes devem selecionar novamente um novo slot (linha 25) e o procedimento é reiniciado (linha 7).

Como pode-se perceber, o algoritmo proposto não possui aumento na complexidade computacional em relação ao Algoritmo Q e o método de Schoute apresentados em (EPCglobal, GS1 Inc. , 2015) e (Schoute, 1983), é simples e fácil de implementar, uma vez que funciona como um DFSA no procedimento principal, a função tratadora de colisões é baseada em operações aritméticas básicas e a estimativa é baseada nos comandos do algoritmo Q (C1G2) (EPCglobal, GS1 Inc., 2015). 


\subsubsection{Análise de desempenho}

Nesta seção, a teoria do acesso aleatório (Spiegel et al., 2002) é analisada para provar que o procedimento de estimativa inicial é preciso e que a função tratadora de colisões pode atingir um desempenho desejável através da definição do tamanho inicial de quadro com tamanho 4 .

Primeiramente considera-se $n$ etiquetas que serão identificadas utilizando um tamanho de quadro $L$. Baseado na teoria do acesso aleatório, para um conjunto com $n$ etiquetas as seguintes definições podem ser feitas:

Definição 2. O número de etiquetas alocadas em um dado slot de tempo segue uma distribuição binomial com n experimentos de Bernoulli e $\frac{1}{L}$ de probabilidade de ocupação (Wu et al. , 2013)Chen (2014). A probabilidade de encontrar-se $k$ etiquetas em quaisquer dos slots é dessa forma definido pela Equação (5.6).

$$
p(L, n, k)=\left(\begin{array}{l}
n \\
k
\end{array}\right) \times\left(\frac{1}{L}\right)^{k} \times\left(1-\frac{1}{L}\right)^{n-k}
$$

Definição 3. O número esperado de slots de sucesso, vazio e colisão em um quadro é dado pelas Equações (5.7), (5.8),(5.9) e (5.10).

$$
\begin{gathered}
E(L, n, k)=L \times\left(\left(\begin{array}{l}
n \\
k
\end{array}\right) \times\left(\frac{1}{L}\right)^{k} \times\left(1-\frac{1}{L}\right)^{n-k}\right) \\
E(L, n, 0)=L \times\left(1-\frac{1}{L}\right)^{n} \\
E(L, n, 1)=L \times\left(n \times\left(\frac{1}{L}\right) \times\left(1-\frac{1}{L}\right)^{n-1}\right) \\
E(L, n, k)=L \times(1-p(L, n, 0)-p(L, n, 1))
\end{gathered}
$$

Lema 4. Se $p(L, n, k) \geq 0.6$ então o valor esperado para 5 tentativas do procedimento de estimativa será $\geq 3$ (maioria dos slots de colisão). Isto significa que a estimativa precisa incrementar o valor de $Q$ em uma unidade. Além disso, esta probabilidade é atingida quando 
a relação $\frac{n}{L} \geq 2$ é satisfeita.

Demonstração.

Aplicando $n \cong 2 L$ em $(5.10)$ tem - se $p(L, n, k)=\left(1-\frac{1}{L}\right)^{2 L}-2 \times\left(1-\frac{1}{L}\right)^{2 L-1}$

Buscando-se o limite da função temos que $\lim _{L \rightarrow \infty}\left(1-\frac{1}{L}\right)^{2 L}-2 \times\left(1-\frac{1}{L}\right)^{2 L-1} \cong 0.6$

Corolário 1. O procedimento de estimativa calcula $L$ como um valor no intervalo $\left[Q_{0}, Q_{1}\right]$ onde $Q_{0}$ significa o valor de $Q$ (potência de 2) mais próximo de $n / 2\left(n / 2<Q_{0}\right)$ e $Q_{1}$ (potência de 2) o valor mais próximo de $n\left(n<Q_{1}\right)$.

Demonstração. O valor esperado do número de colisões $E(L, n, k)$, definido em (5.10), é incrementado quando a relação $z=\frac{n}{L}$ cresce. Substituindo $z$ em (5.10) tem-se (5.11). Uma vez que a função de estimativa inicia com um valor baixo $\left(Q=4, L=2^{4}=16\right)$, quando esta relação atinge um valor menor e próximo de $\left(2^{Q}>\frac{n}{2}\right)$ tem-se $p(L, n, k)<0.6$ fazendo com que $E(L, n, k)<3$. Quando esta situação ocorre, é mais provável que o estimado defina o valor de $Q$ no intervalo $\left[Q_{0}, Q_{1}\right]$, baseado nos valores esperados, conforme Equação 5.10.

$$
p(L, n, k)=L \times\left(1-\left(n \times\left(\frac{1}{L}\right) \times\left(1-\frac{1}{L}\right)^{z L-1}\right)-\left(1-\frac{1}{L}\right)^{z L}\right)
$$

Com o objetivo de comparar os resultados analíticos e de simulação ${ }^{4}$ relacionados ao desempenho da função de estimativa, a Figura 5.31 mostra o comportamento do procedimento de estimativa quando a quantidade de etiquetas varia de 100 até 5200.

Como o Corolário 1 prevê, os valores estimados que mais ocorrem (pontos pretos no gráfico) estão situados entre $Q_{0}$ (linha azul) e $Q_{1}$ (linha amarela). As linhas verde (valor real) e vermelha (média) estão quase sobrepostas, confirmando a precisão do estimador. Além disso, utilizando-se a Equação (5.5.1) pode-se calcular que o processo de estimativa representa aproximadamente $1 \%$ do total de slots utilizados. A Figura 5.32 mostra que o número de slots utilizados durante o processo de estimativa converge para um valor pequeno

\footnotetext{
${ }^{4}$ Foram realizados 1000 experimentos para tomar-se a média.
} 


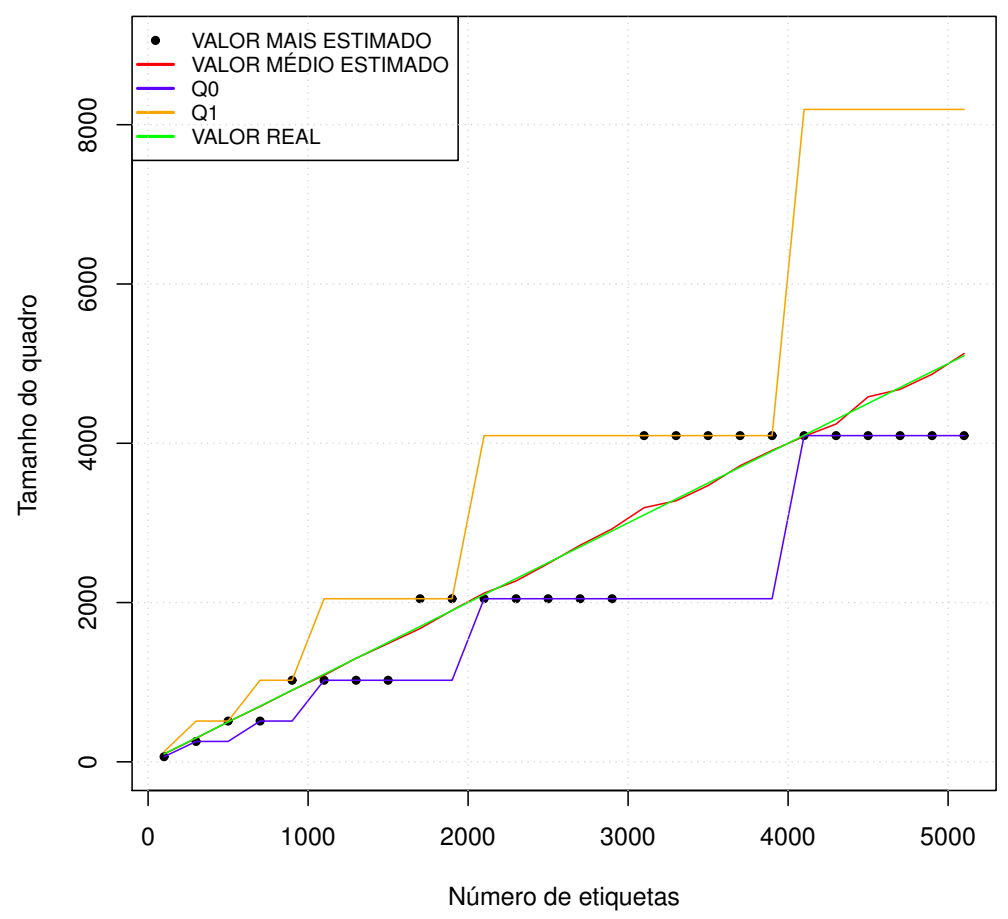

Figura 5.31: Desempenho do procedimento de estimativa.

em relação à quantidade de etiquetas. Em outras palavras, quando aumenta-se a quantidade de etiquetas, o overhead do estimador permanece baixo uma vez que seu aumento ou diminuição ocorrem exponencialmente.

Lema 5. Um tamanho razoável do tamanho do quadro (próximo à quantidade de etiquetas) gera menos etiquetas por slot de colisão. O percentual estimado de 2 ou 3 etiquetas por slot de colisão varia de $77 \%$ até $96 \%$. Esta prévia informação permite a definição de um tamanho inicial de quadro para 4 no procedimento solve_collisions.

Demonstração. Usando-se a Equação (5.6) pode-se obter 18\%, 6\% e 1\% de probabilidade de colisão com 2, 3 e 4 etiquetas respectivamente quando a relação $\frac{n}{L}$ é próxima de 1 . Neste caso 96\% das colisões serão disputadas por duas ou três etiquetas. Se o limite inferior é estimado $\left(L=\frac{n}{2}\right)$, este valor vai a $77 \%$ de colisões com duas ou três etiquetas. Finalmente se o valor médio é estimado $90 \%$ das colisões serão disputadas por duas ou três etiquetas. Independente do valor estimado estar no limite inferior, superior ou ponto médio de $\left[Q_{0}, Q_{1}\right]$, a maioria das colisões ocorrerão entre 2 ou 3 etiquetas, fazendo com que o tamanho de quadro igual a 4 para o procedimento solve_collisions seja um bom valor para aumentar a eficiência da 


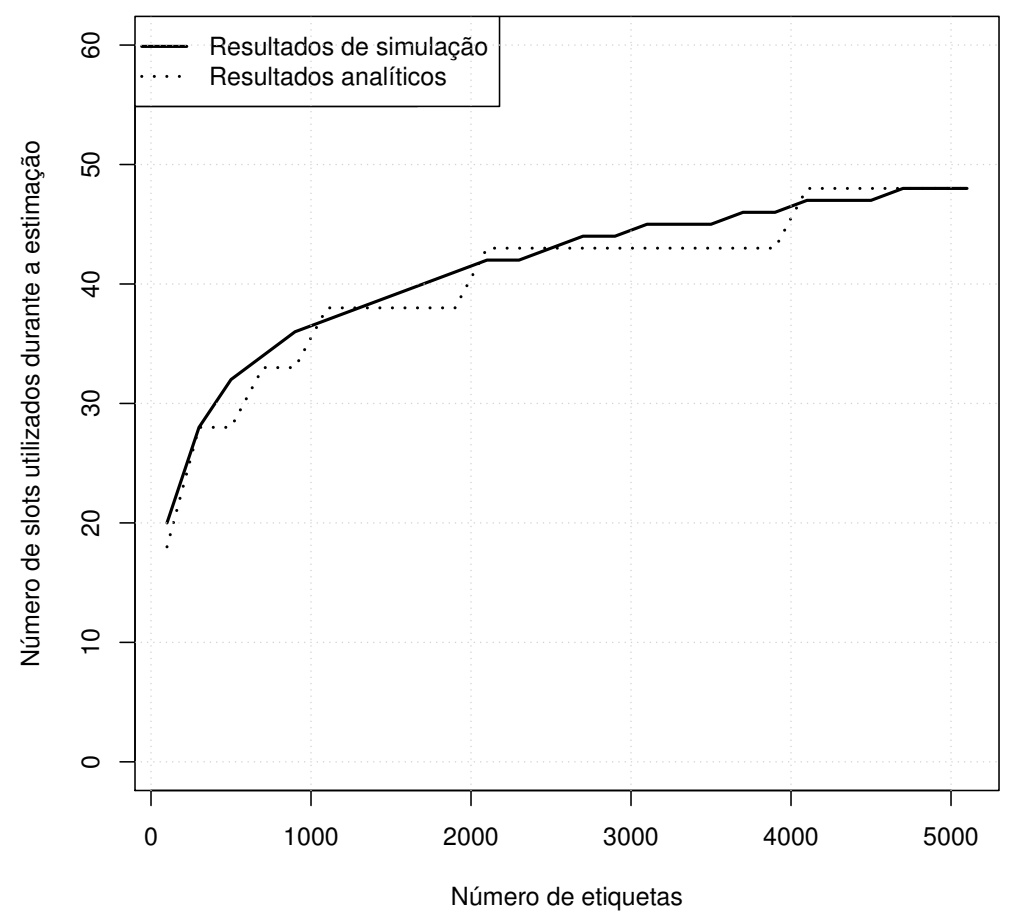

Figura 5.32: Overhead (em slots) do procedimento de estimativa.

identificação.

Lema 6. O valor esperado da eficiência média do sistema do IDFSA-IColR é

$$
S E \cong 0,41
$$

Demonstração. Dado o número total de slots gastos na estimativa $T_{\text {est }}$, o valor estimado do tamanho inicial do quadro $L$, o número de slots utilizados para identificar $k$ etiquetas em colisão $T_{\text {col }}(k)$, o número de slots gastos pelo algoritmo IDFSA-IColR para identificar $n$ etiquetas é dado por (Wu et al., 2013) (Wang et al., 2014)

$$
T(L, n)=T_{\text {est }}+L+\sum_{k=2}^{n} E\left(L, k, c_{k}\right) T_{\text {col }}(k)
$$

$T_{\text {est }}$ é calculado a partir da Equação (5.5.1) e representa aproximadamente $1.5 \%$ de $T(L, n)$.

$$
T_{e s t}=\left(\frac{\ln (n)}{\ln (2)}-4\right) * 5+3
$$


Como $L=1.15 n$ ou $L=0.85 n$, a partir da Equação (5.7), Corolário 1 e Figura 5.33, então (Wu et al., 2013)

$$
T(L, n) \cong T_{\text {est }}+L+1.15(\text { ou } 0.85) L \sum_{k=2}^{n} \frac{T_{\text {col }}(k)}{\times} p((1.15 n \text { ou } 0.85 n), n, k) \cong 2.42 n
$$

Como $S E$ é calculada por $\frac{n}{T(L, n)}$ então temos $\frac{1}{2.428} \cong 0.41$.

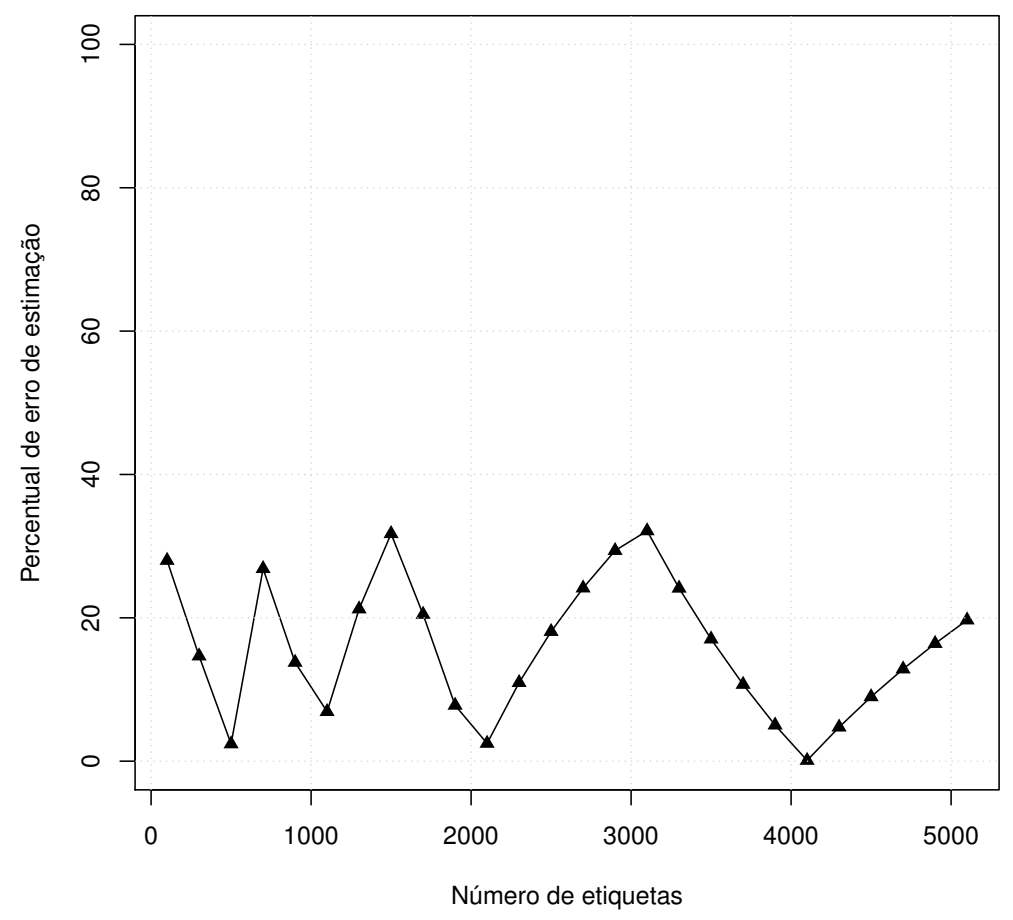

Figura 5.33: Erro de estimativa (\%).

\subsubsection{Parâmetros de simulação}

O sistema consiste de um leitor RFID e $n$ etiquetas sob sua área de cobertura. O modelo do leitor é baseado nas especificações definidas por SkyeTek SkyeModule M9 Compact Flash RFID Reader 9 que opera na frequência de 860-960 MHz, velocidade (data rate) de 40kbps(Namboodiri et al., 2012) e potência em modo leitura $(P)$ (em Watts) de $2250 \mathrm{~mW}$ (Namboodiri et al., 2012) (Klair et al., 2009). 
Os tempos dos slots foram tomados como $8 m s\left(t_{\text {suc }}\right), 1,9 \mathrm{~ms}\left(t_{\text {col }}\right)$ e $0,61 \mathrm{~ms}\left(t_{\text {idl }}\right)$ para os slots de sucesso, colisão e vazio respectivamente. Na prática, a duração dos slots são variáveis e dependem de seu tipo. Dessa forma, os protocolos foram também avaliados levando-se em consideração estas variações com o objetivo de gerar-se também resultados mais confiáveis (Namboodiri et al., 2012). Para fins de comparações com outras propostas, utilizaram-se os valores $0.4 m s\left(t_{\text {suc }}\right), 0.05 m s\left(t_{c o l}\right)$ e $0.05 m s\left(t_{i d l}\right)$, conforme Shakiba et al. (2014), nas comparações na qual a métrica $C_{e f}$ foi levada em consideração.

Assume-se que o leitor RFID fornece energia até que todas as etiquetas sejam identificadas. Apesar das etiquetas serem energizadas simultaneamente, esta tese mede o consumo energético (do leitor) durante o processo de identificação, ignorando o consumo prévio a esse processo (Klair et al., 2009; Namboodiri et al., 2012). Assume-se também que as etiquetas selecionam seus respectivos slots baseados em uma distribuição normal entre 0 e $L-1$ (tamanho do quadro).

Os resultados de simulação que incluem tempo omitem o atraso associado com a energização das etiquetas, propagação e processamento. Além disso a as análises consideram um canal livre de interferência e sem o efeito captura, o que significa que as perdas de pacotes ocorrem devido a colisões apenas. O leitor detecta uma colisão quando a checagem do CRC falha e a confirmação é enviada apenas quando o identificador da etiqueta é recebido sem erros. O tamanho do ID é de 96 bits, incluindo os 16-bits do CRC. Os atrasos devido às confirmações (ACKs) e (CRCs) já estão computados nos tempos dos slots (Klair et al., 2009; Namboodiri et al., 2012). Finalmente, assume-se que as etiquetas são passivas, não possuem fonte de energia própria, não estão em movimento e não sairão da área de cobertura do leitor durante o processo de identificação. Nenhuma nova etiqueta entra na área de cobertura do leitor enquanto um procedimento de identificação não for finalizado.

\subsubsection{Simulações}

O desempenho experimental do algoritmo proposto e dos métodos comparados foram avaliados através de extensivas simulações utilizando-se do método Monte Carlo (Chen , 2014). Para assegurar a convergência dos resultados, cada algoritmo foi simulado 1000 vezes para obter-se a média. Comparou-se o algoritmo proposto com as propostas Schoute 
(Schoute, 1983), lower bound DFSA (Vogt, 2002), Eom-Lee (Eom e Lee, 2010), NEDFSA (Seção 5.3), ODFSA ${ }^{5}$ e Algoritmo Q (C1G2) (EPCglobal, GS1 Inc., 2015). Para fins de comparação com algoritmos de maior complexidade, comparou-se também com os algoritmos Birthday Paradox Estimation - BPE e Bayesian Tag Estimate - BTE (Shakiba et al., 2014; Wu e Zeng, 2010) em relação à métrica $C_{e f}$. Os experimentos foram realizados usando o jRFIDsim.

Todas as simulações foram executadas para uma quantidade de etiquetas variando de 100 a 5200 com variações de 200. O intervalo de confiança foi de 95\% e encontrou-se variações no intervalo de $0.5 \%$ a $0.9 \%$ do valor da média para todos os pontos ${ }^{6}$. O tamanho inicial de quadro foi definido em 16, isto é, valor de $Q=4$, para o Algoritmo Q e DBTSA, 128 para Lower Bound, Schoute, Eom-Lee e ODFSA. A proposta NEDFSA não utiliza tamanho inicial de quadro fixo pois o mesmo faz uma estimativa deste valor. Além disso, o tamanho inicial de quadro do algoritmo proposto também é estimado para um valor próximo da quantidade de etiquetas (no procedimento de estimativa, definiu-se o valor inicial de $Q=4$ ).

Como diferentes métricas podem sinalizar diferentes ganhos ou perdas, os algoritmos foram avaliados em diferentes métricas. Baseado em cada tipo de slot observado (colisões, sucesso ou vazio), após o processo de identificação foram computados o total de slots utilizados $\left(\right.$ Total $\left._{\text {slots }}\right)$, tempo $\left(T_{\text {total }}\right)$ e eficiência do sistema $\left(S_{\text {ef }}\right)$.

Outra métrica importante a ser avaliada é a quantidade de energia desperdiçada durante o processo de identificação das $n$ etiquetas. Conforme mostrado no Capítulo 4, a energia consumida por um leitor RFID para identificar um conjunto de $n$ etiquetas é proporcional ao tempo em modo leitura, em outras palavras, depende do tempo gasto para ler todos os slot usados no processo de identificação (Klair et al., 2009). Dessa forma, utilizando-se dos tempos gastos para leitura de cada tipo de slot, pode-se calcular o consumo total e desperdiçado (Klair et al., 2009).

\footnotetext{
${ }^{5}$ Algoritmo teórico - o leitor conhece a quantidade de etiquetas ao final de cada rodada.

${ }^{6}$ Para facilitar a leitura dos gráficos, não foram incluídos os intervalos de confiança, uma vez que os mesmos são pequenos o suficiente para serem seguramente ignorados.
} 


\subsubsection{Resultados de simulação}

Os resultados obtidos mostram que o correto ajuste do tamanho inicial do quadro pode melhorar o desempenho de diferentes métricas. A seguir serão mostradas comparações abrangentes entre o algoritmo proposto e propostas clássicas da literatura baseadas em resultados experimentais.

i. Eficiência do Sistema e total de slots. A Figura 5.34 mostra a eficiência do sistema $\left(S_{e f}\right)$ de diferentes propostas da literatura. A métrica $S_{\text {ef }}$ denota a relação entre a quantidade de etiquetas e a quantidade de slots utilizados conforme mostrado na Equação 4.2. Pode-se observar que a eficiência dos métodos de Schoute, lower bound, ODFSA, Algoritmo Q e NEDFSA convergem para a estabilidade a medida que a quantidade de etiquetas aumenta. Dentre estes algoritmos, o NEDFSA obtém a mais alta eficiência. Os métodos de Schoute, lower bound e Eom-Lee são menos estáveis devido à definição fixa do tamanho inicial de quadro. No entanto, o algoritmo proposto nesta seção, o IDFSA-IcolR, supera os demais algoritmos e atinge a mais alta eficiência uma vez que utiliza um tamanho inicial de quadro mais razoável e implementa um mecanismo de resolução de colisões mais eficiente. A curva de eficiência do IDFSA-IcolR é próxima de ser horizontal aproximadamente em 0, 41 enquanto todas as demais curvas estão na faixa de 0,30 até 0,39. Os ganhos atingem percentuais de até $3,2 \%, 17 \%$ e $21 \%$ quando comparados respectivamente com o NEDFSA, Algoritmo Q e Schoute. Os resultados mostram também que, a variação do número de etiquetas resultam em diferenças na eficiência para as propostas Lower Bound, Schoute e Eom-Lee, enquanto esta variação pouco afeta ou não afeta o Algoritmo Q, NEDFSA e o IDFSA-IColR.

Além disso pode-se observar que a eficiência do algoritmo proposto é reduzida quando tem-se um número pequeno de etiquetas. A razão para este fato é que quando tem-se poucas etiquetas, o overhead do procedimento de estimativa é mais significativo. A Figura 5.35 exibe a quantidade de slots utilizados em função do número de etiquetas. Nota-se que a quantidade de slots utilizados pelo IDFSA-IcolR é a menor dentre todos os algoritmos comparados, o que é um bom resultado dada a relação direta entre número de slots e tempo de identificação, bem como entre número de slots e consumo de energia.

ii. Tempo de identificação. Como na prática cada tipo de slot possui duração variável, a métrica $S_{\text {ef }}$ nem sempre representa a melhor forma de avaliar um algoritmo anticolisão. As- 


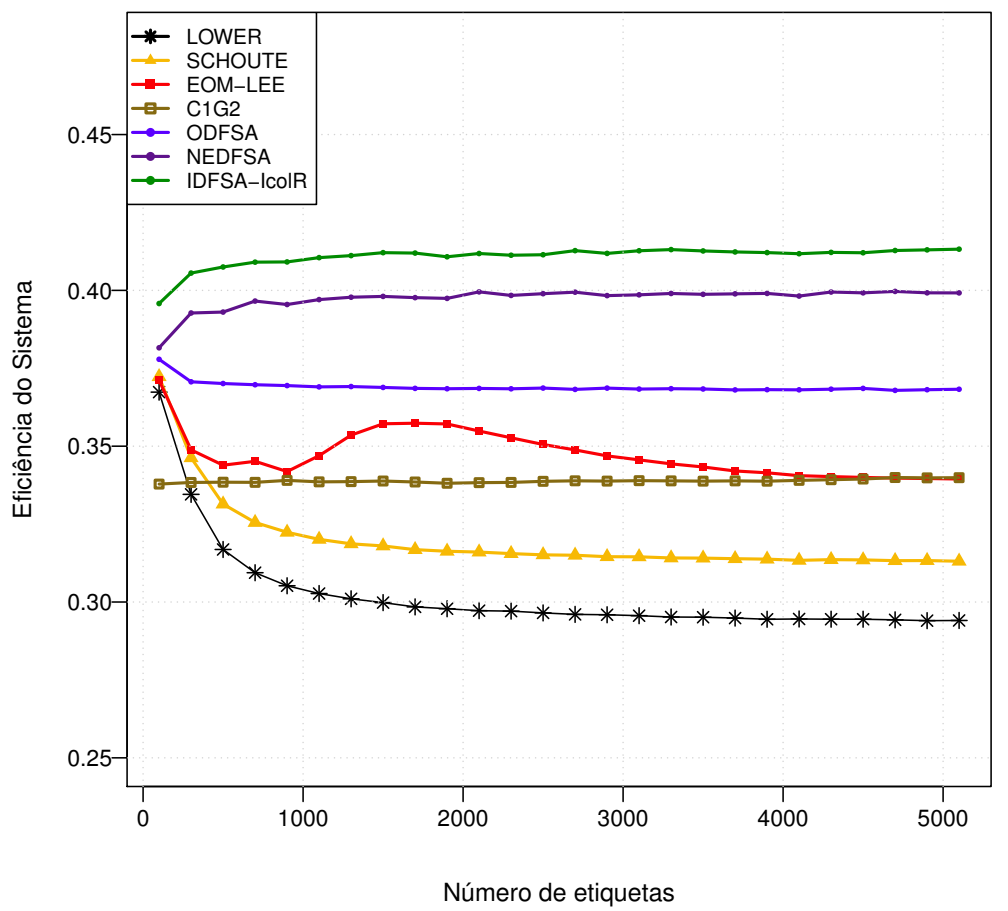

Figura 5.34: Eficiência do Sistema.

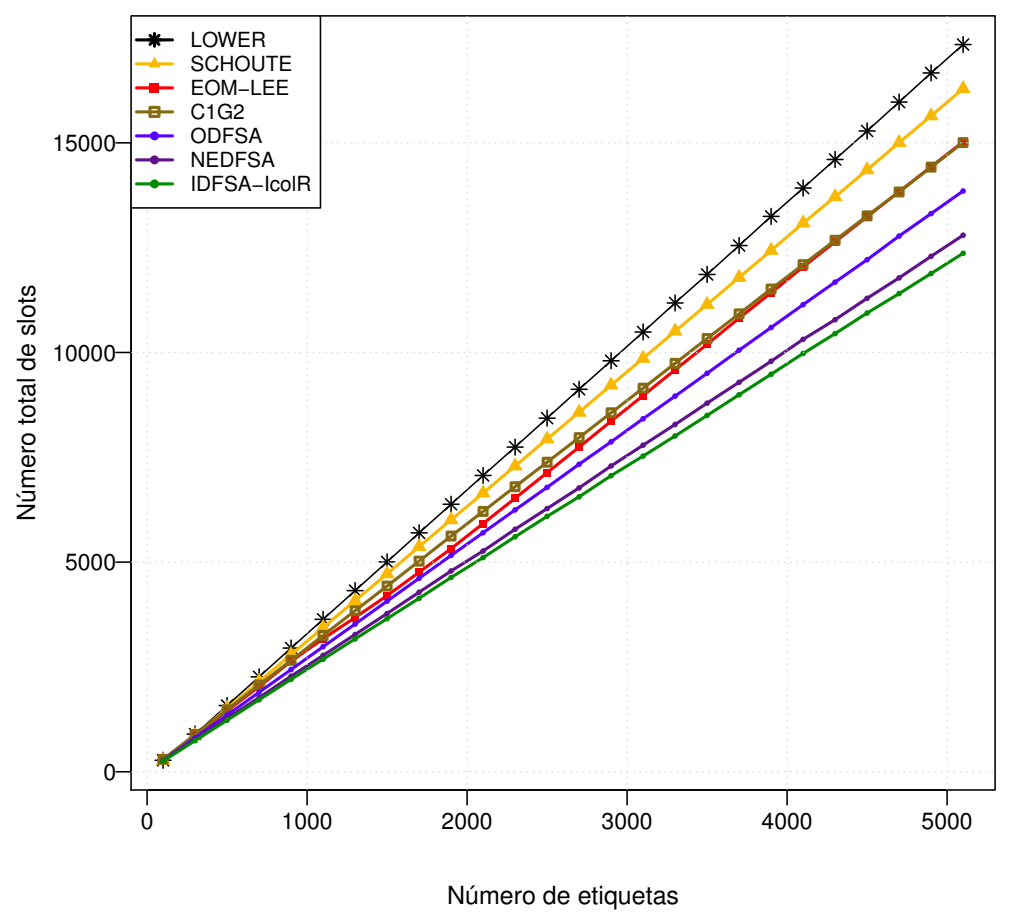

Figura 5.35: Número total de slots utilizados. 
sim, apresenta-se na Figura 5.36 o tempo total de identificação (latência) para a identificação do número de etiquetas definido no eixo $x$.

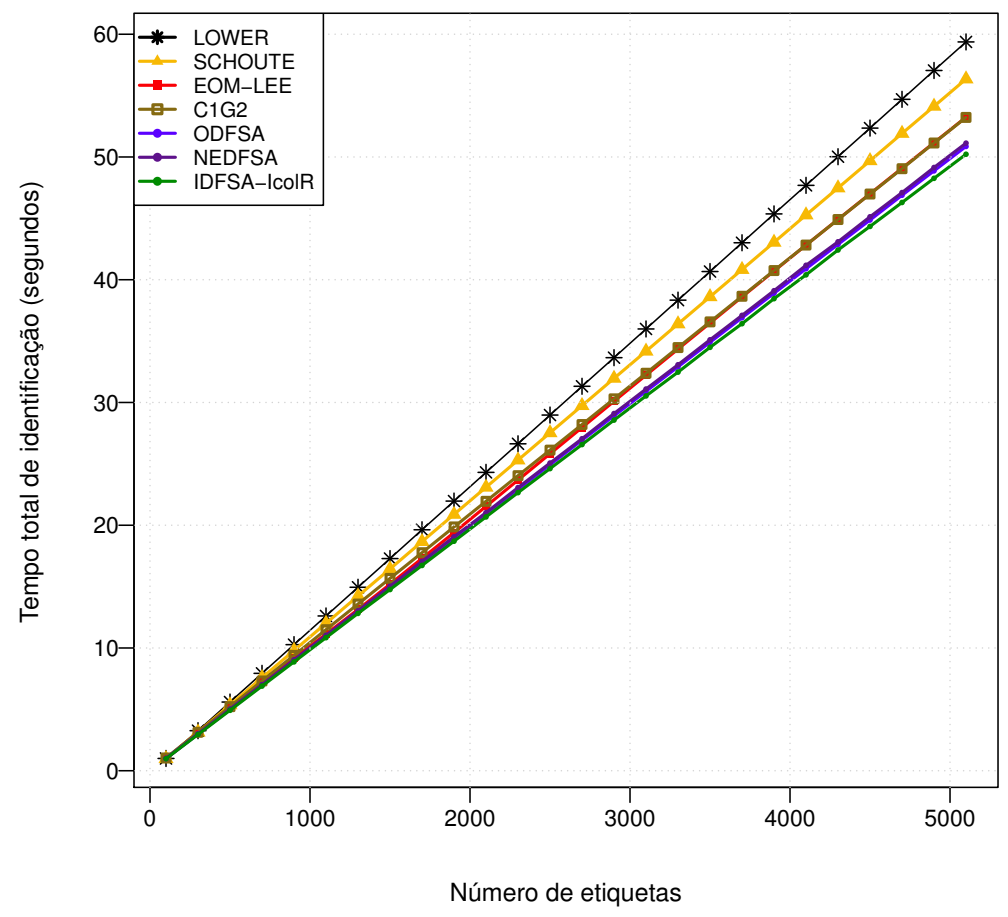

Figura 5.36: Tempo total de identificação.

A figura supracitada mostra que o tempo de identificação dos algoritmos Schoute e lower bound são os maiores, enquanto Eom-Lee e o Algoritmo Q estão na faixa intermediária e o ODFSA, NEDFSA e IDFSA-IColR possuem os mais baixos tempos. O algoritmo proposto identifica o conjunto de etiquetas no menor tempo, aproximadamente $5,7 \%$ e $9,7 \%$ melhor que o Algoritmo Q e Schoute respectivamente. Por exemplo, o IDFSA-IColR pode identificar um conjunto de 3900 etiquetas em aproximadamente 38 s enquanto o Algoritmo Q faria a mesma tarefa em 40s. Se levarmos em consideração vários processos de identificação, por exemplo 100 vezes, o IDFSA-IColR poderia economizar 200 segundos, economia esta que poderia economizar também energia e bateria no caso de leitores móveis.

iii. Energia desperdiçada. A Figura 5.37 mostra a energia desperdiçada (energia gasta devido a colisões e slots vazios) para os diferentes algoritmos.

Nesta figura pode-se claramente observar que o algoritmo proposto economiza mais energia que os demais comparados. Este fato significa que o IDFSA-IColR produz menos slots 


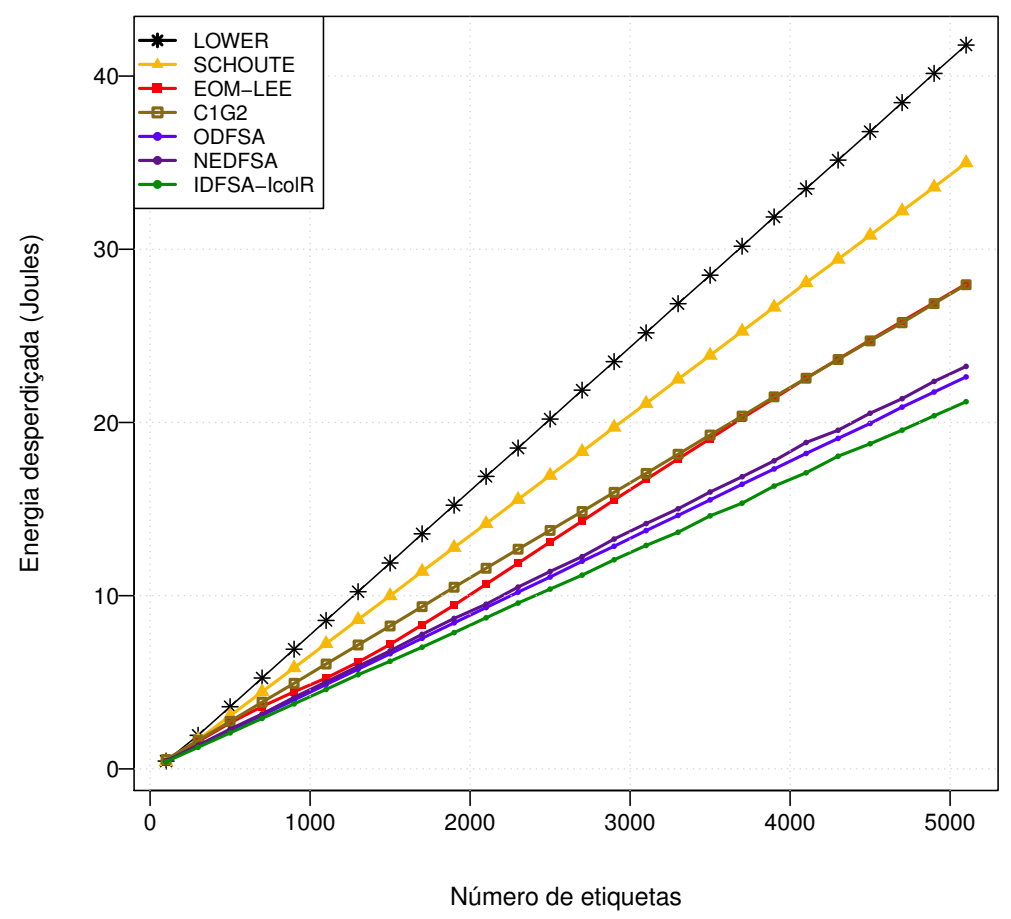

Figura 5.37: Total de energia desperdiçada devido a colisões e slots vazios.

de colisão que os demais (slots de colisão possuem duração maior que os vazios), sendo desta forma mais eficiente em energia, especialmente quando a quantidade de etiquetas aumenta. Por exemplo, na situação em que tem-se 4900 etiquetas a serem identificadas. Utilizando-se o IDFSA-IColR tem-se um desperdício de $20.38 J$ de energia enquanto com o Algoritmo Q desperdiçam-se 26.86 J. Em outras palavras, o algoritmo proposto pode diminuir o consumo de energia em até $24 \%$ comparado com os leitores atuais C1G2.

iv. Eficiência de uso do canal. A Figura 5.38 mostra a métrica $C_{e f}$ das propostas IDFSA-IColR, Birthday Paradox Estimation - BPE e Bayesian Tag Estimate - BTE (Shakiba et al., 2014; Wu e Zeng, 2010). Para o caso desta métrica específica foram utilizados os tempos dos slots de acordo com as definições de Shakiba et al. (2014) (0,4ms-t suc $_{\text {, }}$ 0,05ms- $t_{c o l}$ e $\left.0,05 m s-t_{i d l}\right)$. Como ilustrado na Figura 5.38, o mais alto valor de $C_{e f}$ pertence ao algoritmo IDFSA-IColR com valor de $85 \%$ contra $81 \%$ do BPE e $80 \%$ do BTE. Além disso como a métrica $C_{e f}$ depende to tempo de duração de cada tipo de slot, um valor maior da métrica $C_{e f}$ também implica em economia de energia. 


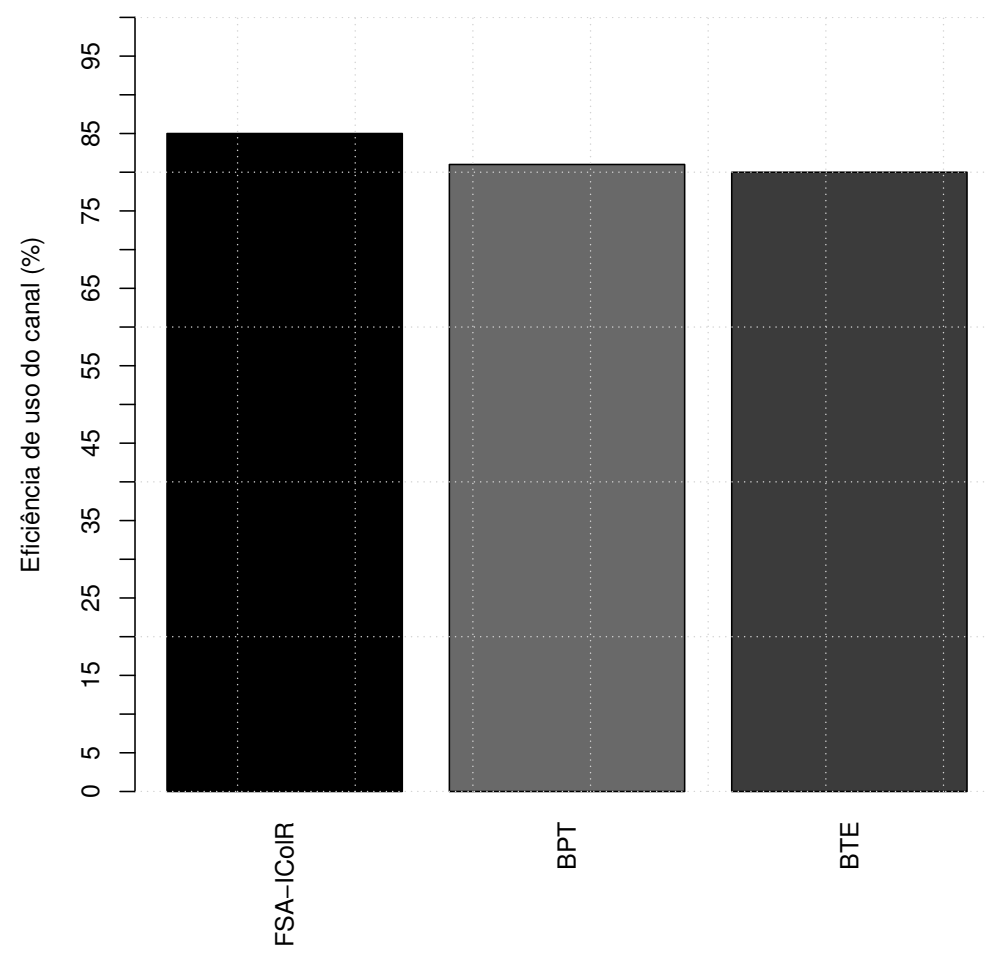

Figura 5.38: Ganhos na eficiência de uso do canal em relação a outras propostas.

\subsubsection{Conclusões parciais}

Nesta seção foi apresentado um algoritmo baseado no padrão DFSA com o objetivo de diminuir a quantidade de colisões e consequentemente o consumo de energia. Desenvolveu-se um algoritmo de estimativa para definição do tamanho inicial de quadro para a melhoria da eficiência geral do sistema. Então definiu-se um procedimento principal com resolução imediata de colisões utilizando quadros com tamanho 4, valor este baseado na análise da teoria do acesso aleatório. Resultados de simulação mostraram que a quantidade total de slots do algoritmo proposto foi a menor em comparação com as demais propostas. Além disso, o IDFSA-IColR consegue diminuir em até $24 \%$ o tempo e energia desperdiçados em comparação com o Algoritmo Q (C1G2) além de ter o custo de redução de apenas $5 \%$ na métrica $S_{\text {ef }}$ em relação ao máximo teórico da categoria BTSA (Wu et al., 2013). 


\subsection{Um algoritmo anticolisão para sistemas RFID sob o efeito captura}

Muitos algoritmos anticolisão (Chen, 2015, 2014; Cui e Zhao, 2009, 2010; Eom e Lee , 2010; He e Wang, 2013; Schoute, 1983; Wu et al., 2013) (Seções 5.3 e 5.5) assumem a existência de três possíveis tipos de slots: sucesso, vazio e colisão. No entanto esta suposição na prática pode ser inválida (Wu e Zeng, 2015). O sinal de uma etiqueta mais próxima do leitor pode ser corretamente decodificada mesmo na situação na qual outras etiquetas transmitam seus identificadores ao mesmo tempo. Dessa forma, as respostas de duas ou mais etiquetas não consistirão necessariamente em uma colisão. Chama-se esta situação de fenômeno do efeito captura (Maguire e Pappu, 2009).

Para o problema do efeito captura, algumas soluções estão propostas na literatura (Cerciello et al. , 2014; Choi et al., 2012; Li e Wang, 2011; Maguire e Pappu, 2009; Natali et al., 2010; Wu e Zeng , 2015). No entanto, estas soluções possuem problemas relacionados a eficiência devido aos seguintes fatores: primeiramente, quando o efeito captura ocorre, a(s) etiqueta(s) não identificada(s) escolherão outro slot para responder em quadros subsequentes, o que leva à ocorrência excessiva de slots de colisão. Além disso, o dimensionamento do tamanho do próximo quadro é prejudicado, uma vez que slots que deveriam ser considerados como colisão passam a ser identificados como sucesso, prejudicando o cálculo do backlog. No caso da ocorrência do efeito captura, os mecanismos anticolisão devem não apenas basear-se na quantidade de etiquetas mas também na probabilidade de ocorrência do efeito captura. Como geralmente esta probabilidade é relacionada ao ambiente de identificação, este valor não é fácil de ser obtido (Bekkali et al., 2015).

O algoritmo apresentado na Seção 5.5 não pode ser utilizado em ambientes onde o efeito captura ocorre, ocasionando a não identificação de um percentual de etiquetas. Isso acontece devido a identificação de um slot de colisão como sendo de sucesso, evitando a chamada do procedimento de resolução de colisões. Esta seção estende a proposta IDFSA-IcolR da Seção 5.5 para o ambiente do efeito captura propondo assim o novo algoritmo chamado de IDFSAIcolR-EC. O algoritmo proposto primeiramente estima o tamanho inicial de quadro baseado no mesmo procedimento apresentado na Seção 5.5. Posteriormente o mesmo chama slot 
por slot. Quando um slot de sucesso é identificado, o leitor sempre envia um ACK para a etiqueta identificada (EPCglobal, GS1 Inc., 2015). No caso de um efeito captura, uma ou mais etiquetas que responderam no mesmo slot detectarão que o ACK não pertence a elas e imediatamente informam ao leitor, que identifica o efeito captura. Dessa forma o leitor então inicia o procedimento resolvedor de colisões com tamanho inicial de quadro (um). Como a maioria dos slots de colisão são disputados por duas etiquetas, consegue-se assim uma diminuição de cerca de $27 \%$ de colisões, de acordo com a teoria do acesso aleatório.

\subsubsection{O IDFSA-IcolR-EC}

O algoritmo de estimativa, primeira fase do IDFSA-IcolR-EC, é o mesmo do IDFSAIcolR e funciona como a seguir: o leitor consulta as etiquetas 5 vezes. Se o leitor detecta pelo menos 3 slots de colisão (provavelmente o valor de Q é pequeno), então o valor de $Q$ é incrementado em uma unidade e o procedimento é reiniciado. Caso contrário, se o leitor recebe pelo menos 4 slots vazios e menos que 2 colisões (provavelmente o valor de Q é grande), então o valor de Q é decrementado em uma unidade e o procedimento é também reiniciado. Caso contrário o algoritmo entra na fase de confirmação. O leitor então consulta as etiquetas 3 vezes. Se o número de slots de colisão for maior do que 1 (provavelmente um ponto médio) então o procedimento retorna o valor médio entre $Q$ e $Q+1$. Caso contrário o atual valor de Q é retornado, pois provavelmente este valor é o mais próximo da quantidade real de etiquetas.

Após a conclusão da estimativa do tamanho inicial de quadro (linha 1 - Algoritmo 11), a segunda fase do IDFSA-IcolR-EC é iniciada (Algoritmo 11). O leitor então começa a consultar cada um dos slots (laço das linhas 3-20). Ao identificar um slot de colisão (linhas 16-19), o leitor chama um procedimento de resolução de colisões (linha 18) que irá identificar as etiquetas do slot atual antes de chamar os demais slots do quadro principal. Uma vez que a maioria das colisões ocorre com a disputa entre duas ou três etiquetas e assume-se a ocorrência de efeito captura, o tamanho inicial de quadro do procedimento de resolução de colisões é definido com tamanho três (linha 18), ao invés de quatro. Já no caso de um slot de sucesso ser identificado (linhas 5-11), o leitor envia um ACK para identificar a etiqueta decodificada (linha 6). Caso o leitor receba uma ou mais respostas logo em seguida 
ao envio do comando ACK, o mesmo detecta a ocorrência de um efeito captura. Baseado na probabilidade de que a maioria das colisões ocorre entre duas etiquetas, e se uma foi identificada pelo efeito captura, então o leitor chama o procedimento resolvedor de colisões com tamanho inicial de quadro igual a 1 (linha 9), obtendo assim a melhor eficiência possível nestes casos.

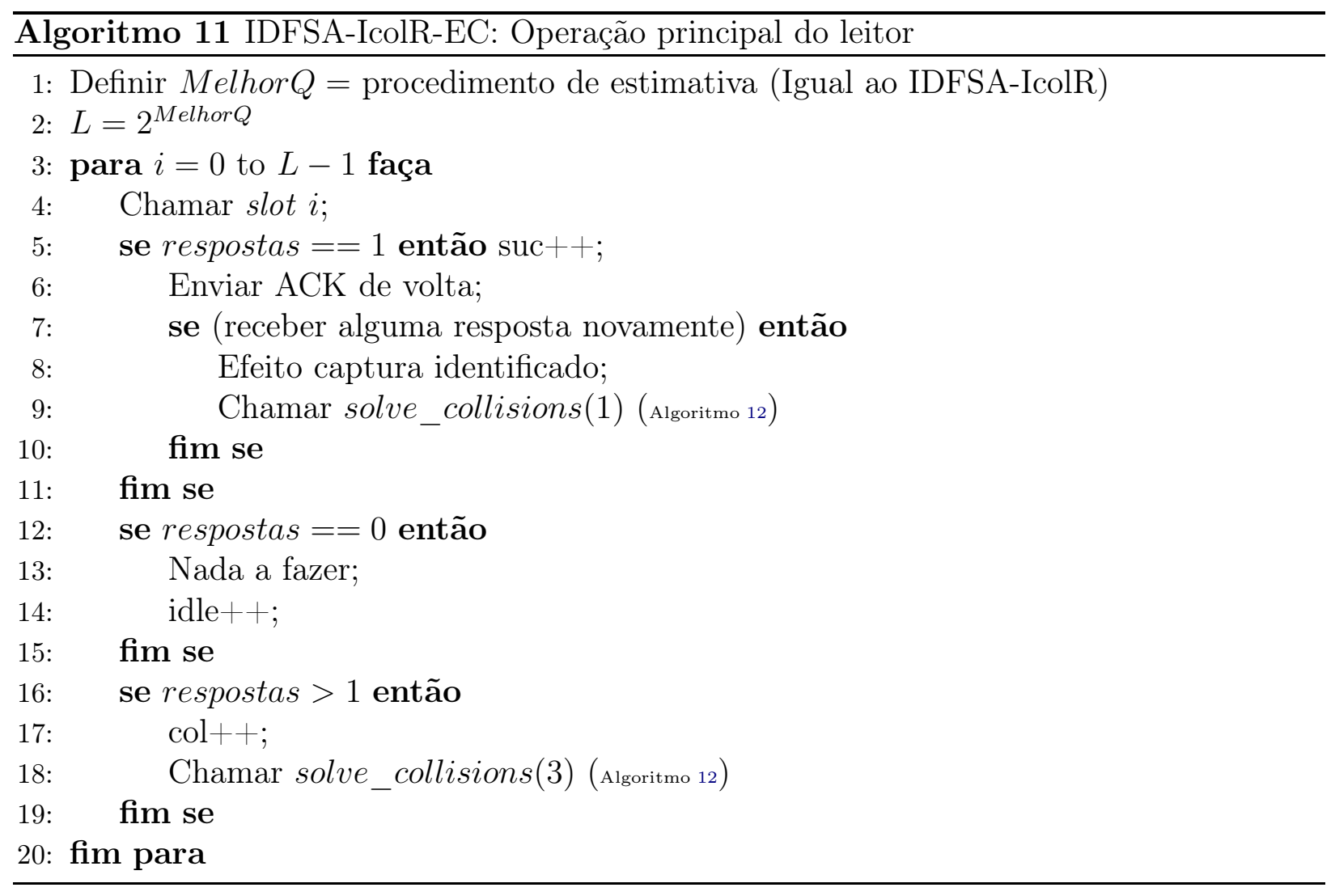

O procedimento de resolução de colisões (Algoritmo 12) funciona da mesma forma do IDFSA-IcolR apresentado na Seção 5.5, com a diferença que o mesmo inclui instruções para lidar com o efeito captura (linhas 14-17). Caso o efeito captura seja detectado na resolução de colisões (o leitor recebe respostas logo após o envio do ACK - linha 14), o leitor registra na variável ec o valor $T R U E$ (linha 16), para que o cálculo do tamanho do próximo quadro seja adequadamente computado (linhas 25-30).

Para que os Algoritmos 11 e 12 funcionem adequadamente, as etiquetas devem passar por pequenas modificações em seu mecanismo de funcionamento, conforme ilustra o Algoritmo 13. Uma etiqueta que responde no slot atual e recebe um ACK direcionado para outra etiqueta deve imediatamente responder ao leitor informando o ocorrido (linha 7). 

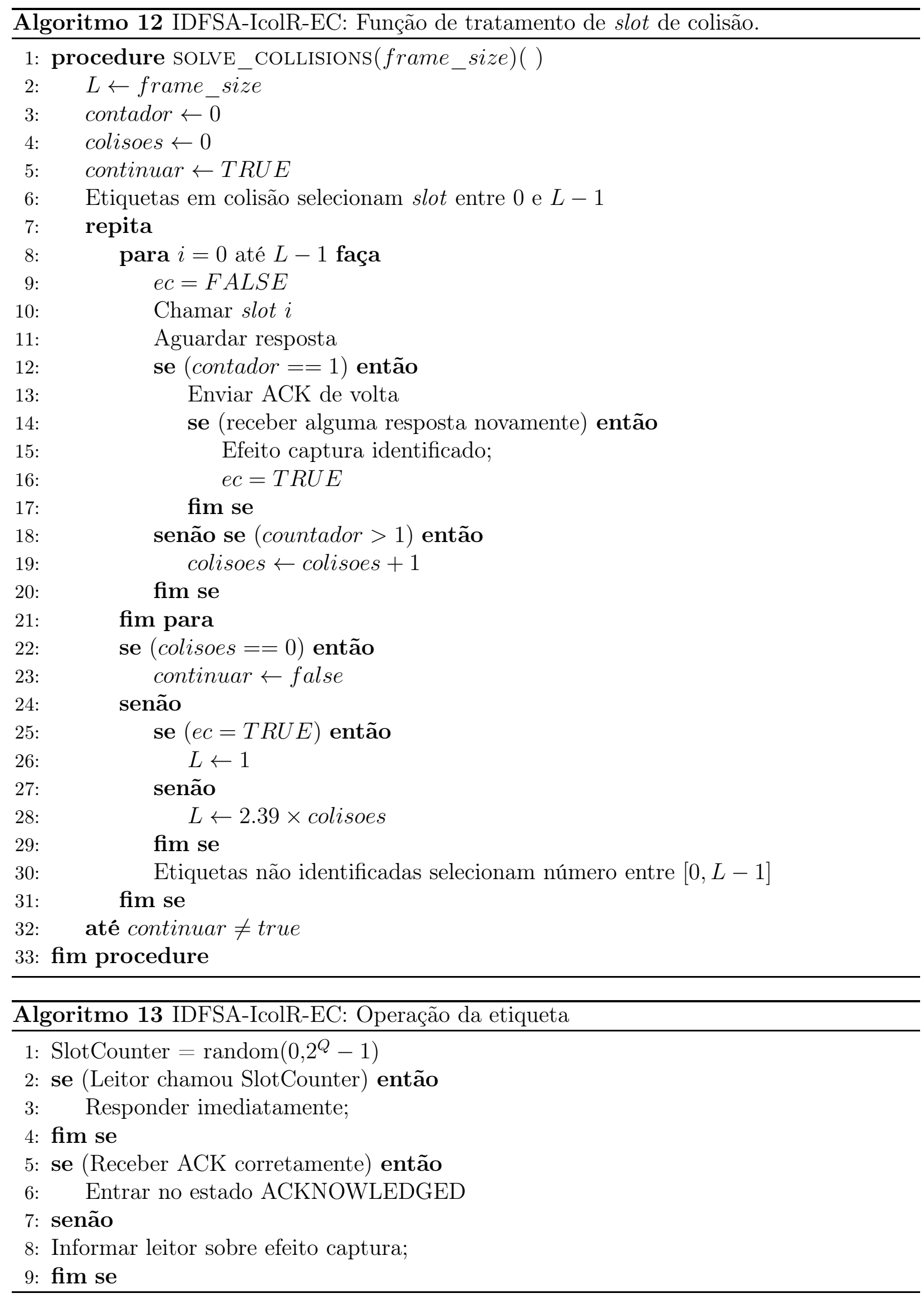


\subsubsection{Parâmetros de simulação}

O sistema consiste de um leitor RFID e $n$ etiquetas sob sua área de cobertura. O modelo do leitor é baseado nas especificações definidas por SkyeTek SkyeModule M9 Compact Flash RFID Reader 9 que opera na frequência de 860-960 MHz, velocidade (data rate) de 40kbps(Namboodiri et al., 2012) e potência em modo leitura $(P)$ (em Watts) de $2250 \mathrm{~mW}$ (Namboodiri et al., 2012) (Klair et al., 2009).

Os tempos dos slots considerados como $1,375 m s\left(t_{S_{1}}\right), 0,3375 m s\left(t_{S_{k}}\right)$ e $0,0675 m s\left(t_{S_{0}}\right)$ para os slots de sucesso, colisão e vazio respectivamente (Chen, 2015).

Assume-se que o leitor RFID fornece energia até que todas as etiquetas sejam identificadas. Apesar das etiquetas serem energizadas simultaneamente, esta tese mede o consumo energético (do leitor) durante o processo de identificação, ignorando o consumo prévio a esse processo (Klair et al., 2009; Namboodiri et al., 2012). Assume-se também que as etiquetas selecionam seus respectivos slots baseados em uma distribuição normal entre 0 e $L$ (tamanho do quadro).

Os resultados de simulação que incluem tempo omitem o atraso associado com a energização das etiquetas, propagação e processamento. Além disso as análises consideram um canal livre de interferência, o que significa que as perdas de pacotes ocorrem devido a colisões apenas. O efeito captura considerado varia de 5 a $56 \%$, de acordo com a quantidade de etiquetas em colisão (conforme Tabela 5.7 da subseção seguinte). O leitor detecta uma colisão quando a checagem do CRC falha e a confirmação é enviada apenas quando o identificador da etiqueta é recebido sem erros. O tamanho do ID é de 96 bits, incluindo os 16-bits do CRC. Os atrasos devido às confirmações (ACKs) e (CRCs) já estão computados nos tempos dos slots (Klair et al., 2009; Namboodiri et al., 2012). Finalmente, assume-se que as etiquetas são passivas, não possuem fonte de energia própria, não estão em movimento e não sairão da área de cobertura do leitor durante o processo de identificação. Nenhuma nova etiqueta entra na área de cobertura do leitor enquanto um procedimento de identificação não for finalizado. 


\subsubsection{Simulações}

Os experimentos foram realizados através de simulação computacional. Os algoritmos foram implementados em linguagem R (R Core Team, 2013), versão 3.0.2. O desempenho experimental do algoritmo proposto e dos métodos comparados foram avaliados através do método Monte Carlo (Chen, 2014). Os simuladores nsRFIDsim e jRFIDsim não foram utilizados pois no momento da escrita desta tese ainda não possuiam o conceito de efeito captura implementado. Para assegurar a convergência dos resultados, cada algoritmo foi simulado 500 vezes para obter-se a média. Comparou-se o algoritmo proposto com o Algoritmo Q (C1G2) (EPCglobal, GS1 Inc., 2015), e ABTSA (Wu e Zeng, 2015), uma vez que o Algoritmo Q é o atual padrão utilizado em sistemas RFID passivos e o ABTSA é um dos algoritmos da mesma taxonomia mais eficiente conhecido da literatura que trata do efeito captura.

Todas as simulações foram executadas para uma quantidade de etiquetas variando de 100 a 4000 com variações de 500. O intervalo de confiança estatístico foi de $95 \%$ e encontrou-se variações no intervalo de $0.3 \%$ a $0.8 \%$ do valor da média para todos os pontos ${ }^{7}$. O tamanho inicial de quadro foi definido em 16, isto é, valor de $Q=4$, para o Algoritmo Q e ABTSA. Além disso, o tamanho inicial de quadro do algoritmo proposto também é estimado para um valor próximo da quantidade de etiquetas (no procedimento de estimativa, definiu-se o valor inicial de $Q=4)$.

Para avaliar o desempenho dos algoritmos, consideraram-se as seguintes métricas utilizadas nas simulações do IDFSA-IColR na seção 5.5.

O efeito captura foi simulado a partir dos parâmetros que mais se aproximam de cenários reais, de acordo com Bekkali et al. (2015). Dessa forma, considerou-se que as probabilidades de ocorrência do efeito captura variam de $5 \%$ a $56 \%$, de acordo com a quantidade de etiquetas por slot de colisão. Quanto menor a quantidade de etiquetas, maior probabilidade de ocorrer o efeito captura (Sanchez e Smith, 1999; Zorzi, 1997). Baseado nas definições de Zorzi (1997), a Tabela 5.7 mostra as probabilidades de acordo com o tamanho da colisão.

\footnotetext{
${ }^{7}$ Para facilitar a leitura dos gráficos, não foram incluídos os intervalos de confiança, uma vez que os mesmos são pequenos o suficiente para serem seguramente ignorados.
} 
Tabela 5.7: Probabilidades de efeito captura baseado na quantidade de etiquetas envolvidas na colisão.

\begin{tabular}{|c|c|}
\hline Quantidade & Probabilidade \\
\hline 2 & $56 \%$ \\
\hline 3 & $19 \%$ \\
\hline 4 & $5 \%$ \\
\hline$>4$ & $0 \%$ \\
\hline
\end{tabular}

\subsubsection{Resultados}

As Figuras 5.39 e 5.40 apresentam a eficiência do sistema e o total de slots respectivamente, para o IDFSA-IcolR-EC e o Algoritmo Q (C1G2). Para todas as quantidades de etiquetas o IDFSA-IcolR-EC supera o Algoritmo Q, sendo que os ganhos são menores quando a quantidade de etiquetas está próxima de 100. Este fato é justificado pelo fato do algoritmo de estimativa produzir uma sobrecarga maior em relação à quantidade total de slots utilizados. Os ganhos percentuais em relação a estas métricas atingem valores aproximados de até $12 \%$. Algumas razões justificam estes ganhos. Primeiramente, o IDFSA-IcolR-EC inicia com um tamanho de quadro mais razoável para identificação do conjunto de etiquetas. Além disso, boa parte das colisões que são identificadas como efeito captura são automaticamente convertidas em sucesso (colisões com duas etiquetas em que ocorre o efeito captura), o que aumenta o desempenho de identificação, uma vez que diminui-se a quantidade total de colisões.

As métricas anteriores não levam em consideração o fato de que a duração em tempo de cada tipo de slot é diferente. Dessa forma, um algoritmo que produza menos colisões tem maior chance de ser mais eficiente, desde que o mesmo não produza slots vazios excessivos. Para medir o desempenho do IDFSA-IcolR-EC e o Algoritmo Q considerando os tempos dos slots, a Figura 5.41 apresenta a comparação dos algoritmos em relação à métrica eficiência de uso do canal (Chen, 2015; Shakiba et al., 2014). Pode-se observar claramente que o IDFSAIcolR-EC também supera o Algoritmo Q. Os ganhos médios chegam a $6 \%$ de melhoria.

Este fato pode ser justificado pelo fato de que o IDFSA-IcolR-EC gera muito menos slots de colisão que o Algoritmo Q, conforme mostra a Figura 5.42. O Algoritmo Q gera em média, $50 \%$ a mais de colisões, o que leva o mesmo a ser $6 \%$ menos eficiente que o IDFSA-IcolR-EC, quando consideramos os tempos dos slots. 


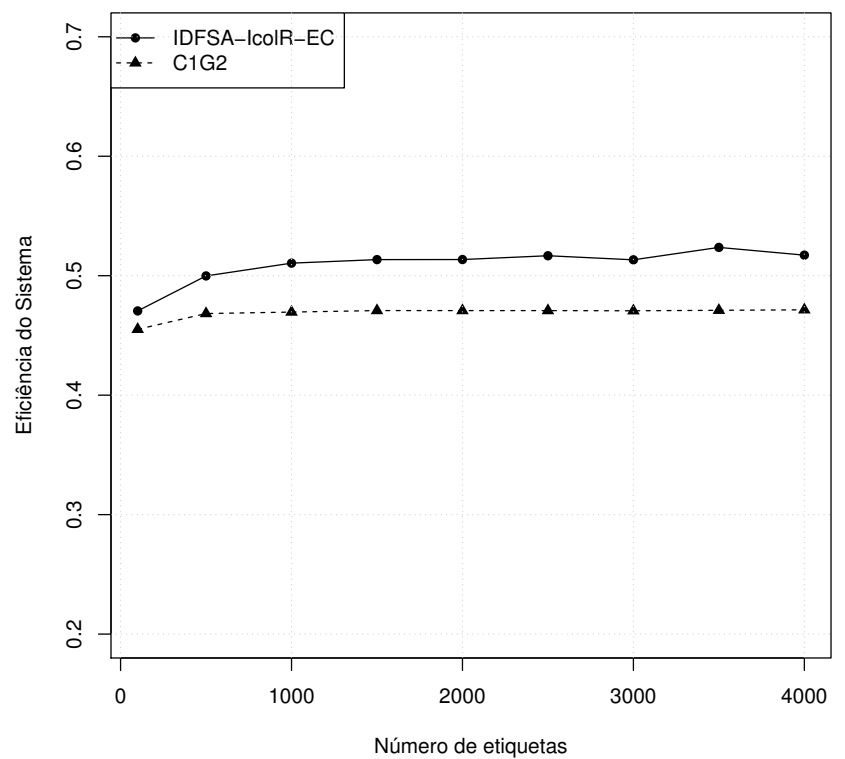

Figura 5.39: Eficiência do Sistema.

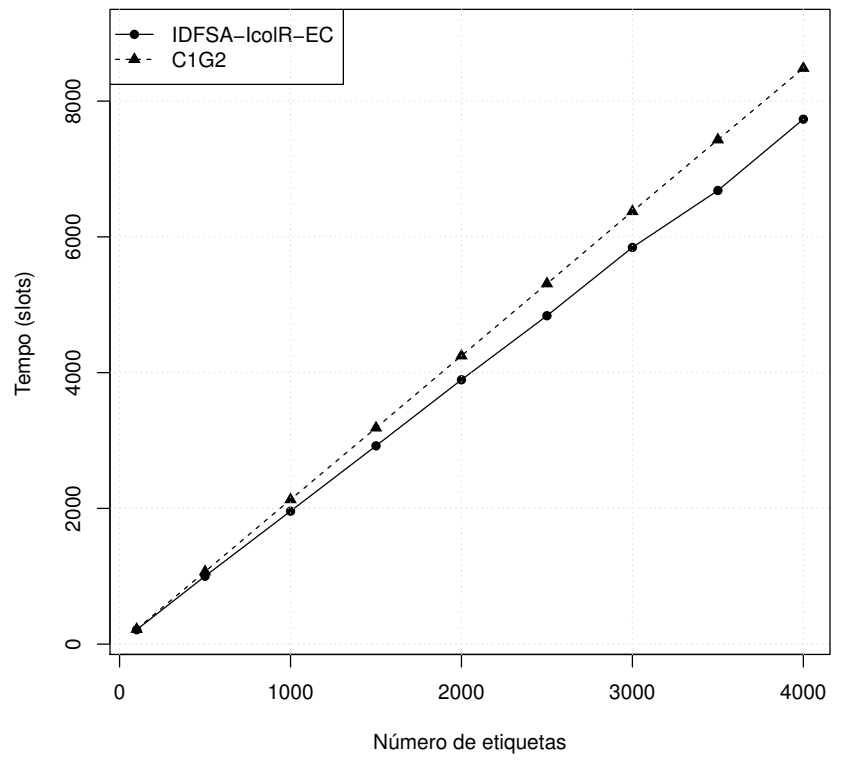

Figura 5.40: Total de slots.

A Figura 5.43 complementa a Figura 5.42 mostrando o total de slots desperdiçados (soma dos slots vazios e de colisão). O Algoritmo Q produz até $25 \%$ mais slots desperdiçados.

Como o consumo de energia está diretamente ligado ao tempo de identificação, os ganhos em consumo de energia do IDFSA-IcolR-EC em relação ao Algoritmo Q também atingem os 6\% aproximadamente, conforme mostra a Figura 5.44. Por exemplo, quando temos 4000 


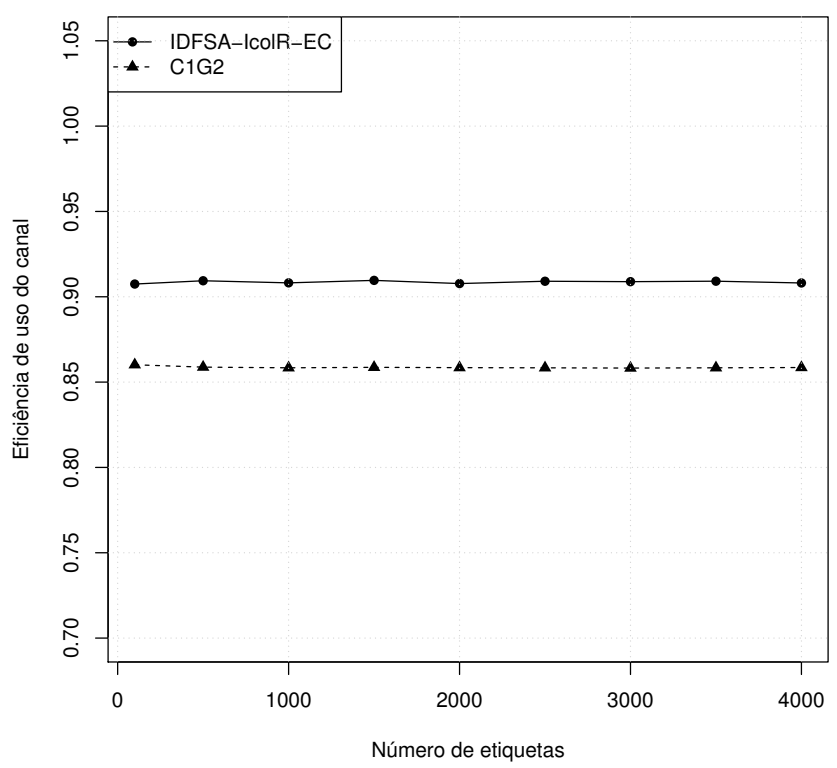

Figura 5.41: Eficiência de uso do canal (\%).

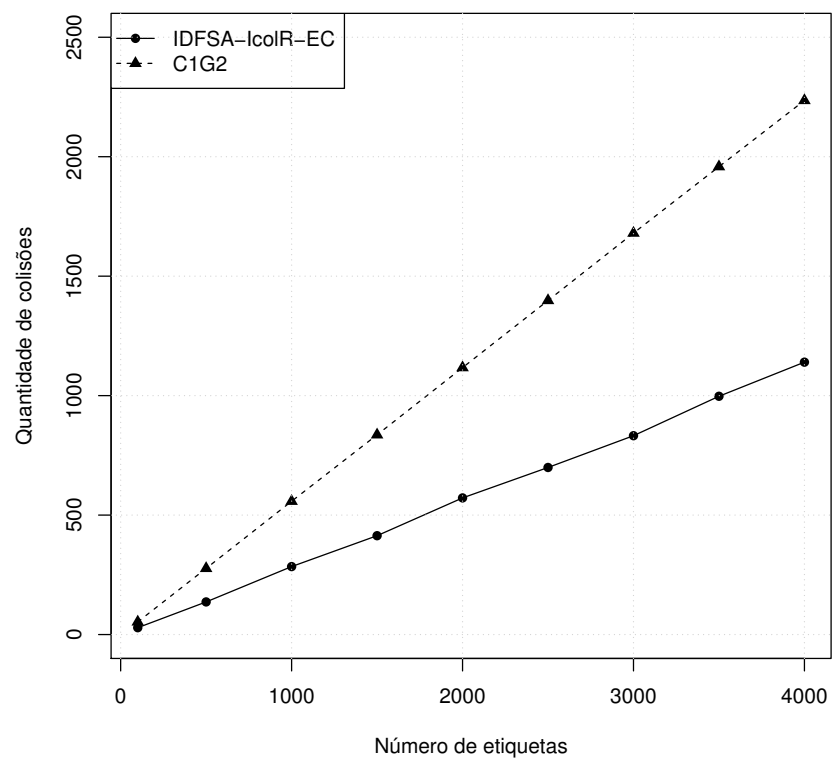

Figura 5.42: Quantidade de colisões.

etiquetas, o leitor utilizando o algoritmo Q gasta cerca de 6,41 Joules enquanto o IDFSAIcolR-EC gasta para as mesmas 4000 etiquetas 6,06 Joules.

A Figura 5.45 apresenta a métrica tempo total para identificação do conjunto de etiquetas para o IDFSA-IcolR-EC, Algoritmo Q e o ABTSA (Wu e Zeng, 2015). O ABTSA foi incluído nesta métrica pois o mesmo forneceu informações suficientes apenas para esta métrica. O 


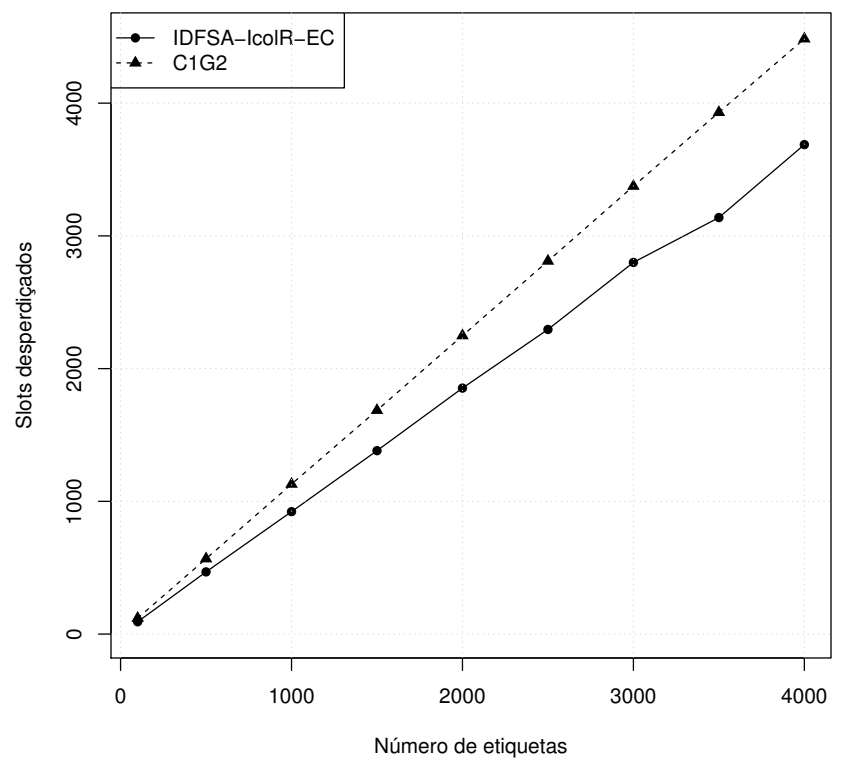

Figura 5.43: Slots desperdiçados.

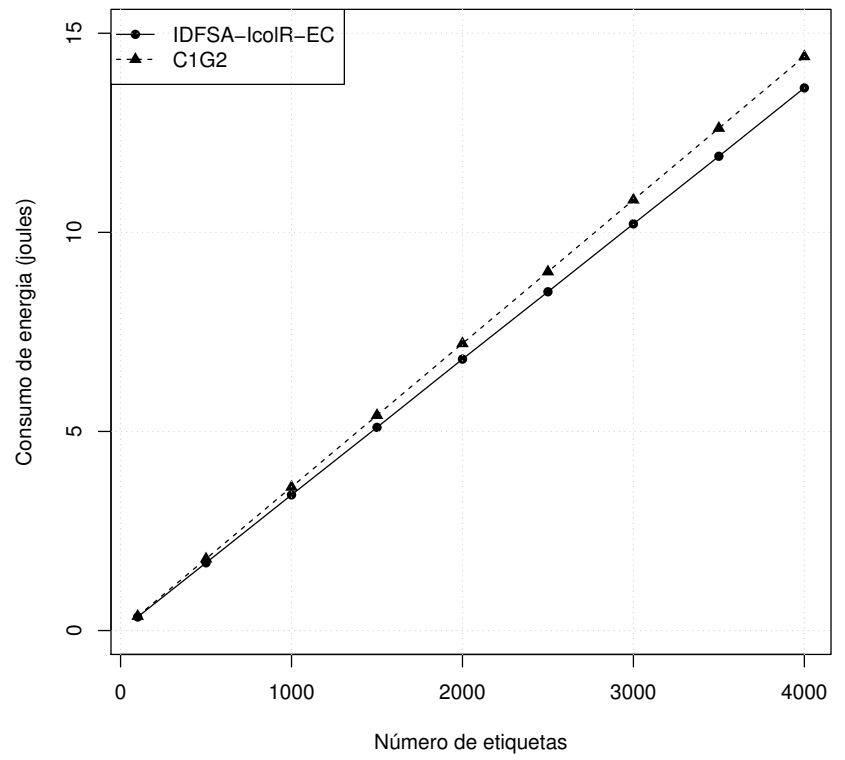

Figura 5.44: Consumo de energia (Joules).

ABTSA-40 refere-se a ocorrência do efeito captura em $40 \%$ das colisões e o ABTSA-100 em 100\% das colisões. O IDFSA-IcolR-EC supera as demais propostas para todas as quantidades de etiquetas simuladas. O IDFSA-IcolR-EC supera o Algoritmo Q em 6\%, o ABTSA-40 em $51 \%$ e o ABTSA-100 em 35\%. Estes ganhos podem ser justificados pelo fato de que o método ABTSA utiliza um procedimento resolvedor de colisões que gera colisões excessivas, 
mesmo no caso em que a probabilidade de efeito captura é de 100\% (ABTSA-100). Além disso, situações em que o efeito captura é constante não correspondem à situações reais em comunicações sem fio (Sanchez e Smith, 1999; Zorzi, 1997).

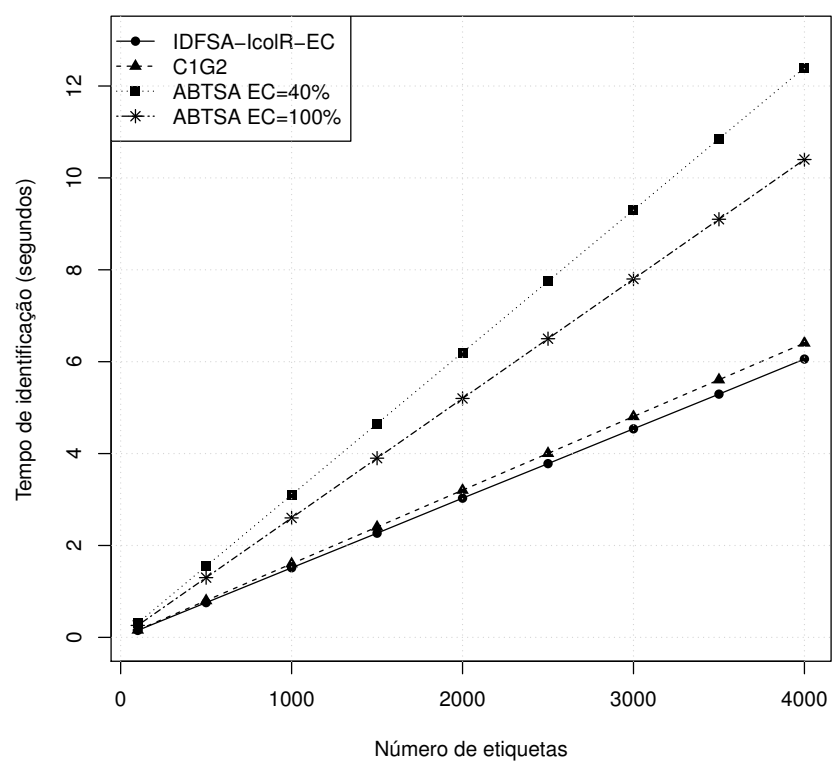

Figura 5.45: Tempo total de identificação.

\subsubsection{Conclusões parciais}

Nesta seção, apresentou-se um novo algoritmo anticolisão para ambientes onde o efeito captura ocorre. O algoritmo proposto, IDFSA-IcolR-EC, adota a estratégia de estimativa do tamanho inicial de quadro para um valor próximo à quantidade de etiquetas. Posteriormente chama cada um dos slots e resolve as colisões assim que as mesmas ocorrem. Resultados de simulação mostraram que o algoritmo proposto supera, em relação à várias métricas, o algoritmo Q (EPCglobal, GS1 Inc., 2015) e o ABTSA (Wu e Zeng, 2015) quando as probabilidades de efeito captura seguem os parâmetros e definições apresentados por Bekkali et al. (2015); Sanchez e Smith (1999); Zorzi (1997). 


\section{Capítulo 6}

\section{Conclusões}

Esta tese conduziu um estudo abrangente sobre os protocolos anticolisão baseados em FSA para sistemas RFID. Este tipo de estudo é necessário para a determinação se os atuais algoritmos da literatura são adequados e se podem ser melhorados para a próxima geração de aplicações. Além disso, observou-se que a maioria das propostas da literatura não abordam aspectos como o consumo de energia e tempo de identificação, fatores estes importantes devido à utilização cada vez maior de leitores operados à bateria. A partir do estudo do Capítulo 3, identificou-se uma série de métricas utilizadas para a avaliação de desempenho de protocolos RFID baseados em FSA e chegou-se a novas métricas, como o tempo de identificação e consumo de energia, que podem ser calculados a partir da contagem dos tipos de slots utilizados durante o tempo de identificação. A utilização apenas da eficiência do sistema $\left(S_{e f}\right)$ ou da contagem total de slots muitas vezes pode não ser suficiente para uma justa e correta avaliação de desempenho, uma vez que diferentes algoritmos podem atingir desempenhos semelhantes nas métricas supracitadas, mas com ganhos maiores em outras métricas. Dessa forma, o Capítulo 4 analisou e propôs uma taxonomia de métricas de desempenho que podem ser utilizadas na avaliação de desempenho mais abrangente e correta dos mecanismos anticolisão propostos. Esta tese contribuiu assim para o correto entendimento a respeito de como melhor avaliar os protocolos anti-colisão propostos, uma vez que estes protocolos são responsáveis por aumentar ou reduzir o consumo total de energia de um sistema RFID.

No Capítulo 5 foram apresentados os resultados obtidos com diversos mecanismos an- 
ticolisão novos que constituem as principais contribuições desta tese. Com o objetivo de minimizar o consumo energético em diferentes situações nas quais um sistema RFID é utilizado, quatro mecanismos foram propostos. O primeiro eliminou respostas desnecessárias em cenários de localização e rastreamento. Os dois seguintes, NEDFSA e IDFSA-IcolR, atacaram diretamente o problema chave do aumento do consumo energético em leitores RFID, ou seja, as colisões. Para este fim observou-se que as propostas da literatura possuem uma preocupação maior em como ajustar os tamanhos de quadros intermediários sem preocuparse com o quadro inicial. A partir desta observação propôs-se um novo algoritmo anticolisão (NEDFSA) que ajusta o tamanho do quadro inicial e resolve as colisões com um método parecido com o de Schoute (Schoute, 1983). A abordagem conseguiu melhorar o desempenho em relação ao Algoritmo Q em até 14\%. A partir do estudo da teoria do acesso aleatório, pode-se melhorar os mecanismos de estimação do tamanho inicial do quadro assim como o mecanismo de resolução de colisões, gerando um novo algoritmo, o IDFSA-IcolR, que por sua vez melhorou o desempenho em relação ao Algoritmo Q em até 21\%. Além disso, através da análise do consumo de energia, verificou-se que o IDFSA-IcolR supera também os mais modernos algoritmos anticolisão em termos de consumo de energia (Wu et al., 2013), uma vez que o mesmo gera menos colisões, implicando em diminuição deste consumo. Finalmente após estudo e análise das propostas que lidam com o efeito captura, estendeu-se o algoritmo IDFSA-IcolR para funcionar em ambientes onde ocorre o efeito captura, gerando o IDFSAIcolR-EC, que também eleva o desempenho de identificação em relação ao Algoritmo Q e ao recente ABTSA (Wu e Zeng, 2015). Apesar dos ganhos obtidos com os mecanismos propostos parecerem pequenos, quando consideram-se cenários em que múltiplas leituras (na casa das milhares de leituras) são realizadas por dezenas e centenas de leitores ao redor do mundo, a economia de energia, principalmente em leitores operados a bateria, passa a tornar-se significativa. Este fato reforça a relevância da pesquisa condizida por esta tese.

Além dos quatro mecanismos propostos, dois softwares livres também foram resultados desta tese, servindo como base para o desenvolvimento e avaliação dos mecanismos propostos: O nsRFIDsim, um módulo para simulação de eventos discretos para o simulador ns-2 e o jRFIDsim, uma suíte de benchmark para avaliação de desempenho de algoritmos anticolisão para sistemas RFID. Esta tese, portanto, contribui para avançar o estado da arte acerca de 
novas formas de se minimizar as colisões durante a identificação das etiquetas, bem como contribui com os pesquisadores da área que podem avaliar suas propostas de forma mais padronizada com a utilização de um softwares que podem tornar-se padrão para avaliação de desempenho na área. 


\section{Capítulo 7}

\section{Trabalhos Futuros}

Apesar de tornar a tecnologia RFID mais factível, os protocolos apresentados no Capítulo 3 ainda precisam de esforços em vários aspectos. Este capítulo apresenta os desafios em aberto que ainda necessitam de esforços de pesquisa, com o objetivo de tornar a tecnologia mais robusta e praticável no mundo real. A Tabela 7.1 resume os desafios em aberto à pesquisa e o porquê dos mesmos serem especificamente cruciais para a tecnologia RFID.

\section{$7.1 \quad$ Mobilidade}

As propostas de protocolos anticolisão para sistemas RFID atuais não levam em consideração a movimentação das etiquetas, o que pode causar falhas na identificação devido à mobilidade das mesmas, resultando em uma taxa de identificação reduzida. Também é um aspecto importante de ser analisado com maior profundidade, pois muitos cenários exigem a movimentação dos objetos identificados. Um dos poucos trabalhos que leva em consideração este aspecto é o proposto por Han et al. (2012). No entanto o desafio ainda necessita de esforços para ser melhor entendido e analisado.

\subsection{Múltiplos leitores e ambientes densos}

Sistemas RFID baseados em C1G2 utilizando múltiplos leitores são necessários em muitas situações e cenários, nos quais leitores são posicionados em locais específicos. Neste tipo de cenários, interferências entre leitores podem ocorrer e os mesmos podem degradar 
Tabela 7.1: Desafios em aberto

\begin{tabular}{ll}
\hline Desafio & Descrição \\
\hline Mobilidade & Em cenários que envolvem mobilidade das etiquetas, o \\
& leitor deve rapidamente identificar o conjunto de etique- \\
& tas dentro de um tempo limitado. A maioria dos proto- \\
& colos anticolisão para RFID não levam em consideração \\
& este aspecto importante. (Han et al., 2012).
\end{tabular}

Múltiplos leitores e Por razões de desempenho, cenários que utilizam uma ambientes densos quantidade muito grande de etiquetas geralmente necessitam de mais de um leitor abrangendo a área de cobertura desejada (Dong et al., 2008). O balanceamento de carga entre leitores torna-se assim um problema muito relevante a ser estudado, principalmente devido à emergente Internet das Coisas, que poderá aumentar a quantidade de etiquetas necessárias de forma significativa (Perera et al., 2015).

Interferência

Vários níveis de interferência podem afetar o processo de identificação do leitor (Namboodiri et al., 2012). Assim como o efeito captura, a interferência ocorre em cenários reais, tornando seu estudo também muito importante e relevante, uma vez que poucos estudos tem sido realizado a respeito.

o desempenho geral do sistema. Cenários como este são denominados de ambientes densos (Bueno-Delgado et al., 2013). Portanto, com o objetivo de minimizar as colisões e interferências entre os leitores assim como aumentar a eficiência em termos de energia e tempo, o estudo dos mecanismos que lidam com este tipo de situação ainda necessitam de estudos e esforços para serem melhor investigados, especialmente para reduzir a quantidade de leitores, o que também diminui o consumo energético.

\subsection{Interferência}

A utilização de sistemas RFID em larga escala pode potencialmente resultar em efeitos não desejados, como a interferência de sinal. Como múltiplos leitores e outros equipamentos sem fio podem existir próximos uns dos outros, os sinais que trafegam no meio compartilhado podem interferir uns com os outros, resultando em erros de identificação. Além disso, devido às limitações de potência, propagação e obstáculos físicos, o sinal recebido pelos 
leitores podem tornar-se muito fraco, fazendo com que a perda de pacotes seja inevitável (WANG et al., 2013).

O desempenho da maioria das propostas da literatura é usualmente avaliado em cenários livres de interferências (WANG et al., 2013), mas é bem sabido que este desempenho é degradado em relação à cenários que não são livres de interferências (Zhang et al., 2013). As implicações da interferência descritas por Namboodiri et al. (2012) necessitam ser melhor estudadas e analisadas com o objetivo de se avaliar o desempenho de protocolos anticolisão em cenários mais realísticos, uma vez que cenários livres de erros não são comuns. 


\section{Apêndice A}

\section{Exemplos}

Exemplo A.0.1. Suponha que na identificação de 3100 etiquetas, utilizando o algoritmo de Mota (2015b), sejam produzidas as seguintes informações: Total $_{\text {slots }}=7530 ; \mathrm{col}=2363$; $i d l=2038 ; s u c=3100$. Estimar a eficiência do sistema $\left(S_{\text {ef }}\right)$ e o ganho em relação ao algoritmo Q $\left(G_{q}\right)$ durante a identificação do conjunto de etiquetas. Considere a $S_{e f}=0,34$ para o algoritmo Q.

$$
\begin{gathered}
S_{\text {ef }}=\frac{n}{\text { Total }_{\text {slots }}}=\frac{\text { suc }}{\text { Total }_{\text {slots }}} \\
=\frac{3100}{7530}=0,4116 \\
S_{\text {ef }} \cong 0,412 \\
G_{q}=\mid \frac{S_{e f}^{q}-S_{e f}^{X}}{S_{e f}^{q}|=| \frac{0,34-0,412}{0,34} \mid} \\
=\left|\frac{-0,072}{0,34}\right|=0,2117 \\
G_{q} \cong 21,2 \%
\end{gathered}
$$

Exemplo A.0.2. Suponha que o leitor RFID seja do tipo SkyeModule M8 ${ }^{1}$, com velocidade de $26 k b p s$, corrente em modo scan de $450 m A$ e voltagem de $5 V$ (Klair et al., 2009, p.963).

\footnotetext{
${ }^{1}$ http://www.skyetek.com/docs/commonblade/stpv3guide.pdf. Acessado em Agosto de 2015
} 
Dessa forma temos que a potência de energia para leitura de uma etiqueta $(P=$ $V I(W))$ é de $P=5 \times 0,45=2,25 W$. O valor de $D=\frac{I D}{\text { data-rate }}$ é igual a $D=0,0036 s$. Assim o consumo $E=8,1 m J$.

$$
\begin{gathered}
P=V I(W) \\
P=5 \times 0,45=2,25 \mathrm{~W} \\
D=\frac{I D}{\text { data }- \text { rate }}=\frac{96}{26000}=0,0036 \mathrm{~s} \\
E=2,25 \times 0,0036=8,1 \mathrm{~mJ} \\
E=8,1 \mathrm{~mJ}
\end{gathered}
$$

Exemplo A.0.3. Suponha um leitor móvel possua uma bateria de $1,5 \mathrm{~V}$ e capacidade de $6000 \mathrm{mAh}$. Deseja-se saber qual a energia armazenada $S E$ (em Joules) nesta bateria.

Utilizamos os seguintes cálculos abaixo²:

$$
\begin{gathered}
S E=\left(\frac{(m A h \times V)}{1000}\right) \times 3600=\left(\frac{(6000 \times 1.5)}{1000}\right) \times 3600=\left(\frac{9000}{1000}\right) \times 3600=9 \times 3600=32,4 k J \\
S E=32,4 k J
\end{gathered}
$$

Exemplo A.0.4. Suponha que na identificação de 2600 etiquetas, utilizando-se o algoritmo de Schoute, 1983, foram gastos 8252 slots. Destes, 3400 foram de colisão, 2252 vazios e 2600 de sucesso. Calcular o consumo de energia total $\left(E_{\text {total }}\right)$ e disperdiçado $\left(E_{\text {waste }}\right)$ na identificação deste conjunto de etiquetas. Considerar a utilização do equipamento Skyetek M8 RFID Reader, 5V, $2250 \mathrm{~mW}$ (scan mode) e $26 \mathrm{kbps}$.

\footnotetext{
${ }^{2}$ http://www.rapidtables.com/convert/electric/mah-to-wh.htm
} 


$$
T_{\text {total }}=t_{\text {suc }} \times n+t_{\text {col }} \times c o l+t_{i d l} \times i d l=8 \times 2600+1,9 \times 3400+0,61 \times 2252
$$

$$
=20800+6460+1374=28634 m s \cong 28 s
$$

$$
T_{\text {total }} \cong 28 s
$$

$$
T_{\text {waste }}=t_{c o l} \times c o l+t_{i d l} \times i d l=1.9 \times 3400+0,61 \times 2252
$$

$$
=6460+1374=7834 m s \cong 7,8 s
$$

$$
T_{\text {waste }} \cong 7,8 s
$$

$$
\begin{gathered}
E_{\text {total }} \cong P \times T_{\text {total }}=2250 \times 28 \cong 63000 \mathrm{~mJ}=63 \mathrm{~J} \\
E_{\text {total }} \cong 63 \mathrm{~J} \\
E_{\text {waste }} \cong P \times T_{\text {waste }}=2250 \times 7,8 \cong 17550 \mathrm{~mJ}= \\
E_{\text {waste }} \cong 17,55 \mathrm{~J}
\end{gathered}
$$

Exemplo A.0.5. Suponha que um leitor RFID utilize uma bateria de capacidade igual ao do Exemplo A.0.3. Estimar a quantidade de vezes que o conjunto de etiquetas do Exemplo A.0.4 poderia ser identificado antes de uma recarga.

$$
\text { Autonomia } a_{\text {leituras }} \cong \frac{32400}{63} \cong 514 \text { vezes }
$$

Autonomia $a_{\text {tempo }} \cong 4$ horas de funcionamento em modo leitura (scan)

Exemplo A.0.6. Suponha que um quadro de tamanho 128 esteja definido para identificar 80 etiquetas. Qual o valor esperado para cada um dos tipos de slots (colisão, sucesso e vazio)? 


$$
(\text { Vazio }) E(L, n, 0)=L \times\left(1-\frac{1}{L}\right)^{n}
$$

$128 \times\left(1-\frac{1}{128}^{8} 0\right) \Rightarrow 128 \times 0.534 \cong 68$

(Sucesso $) E(L, n, 1)=L \times\left(n \times\left(\frac{1}{L}\right) \times\left(1-\frac{1}{L}\right)^{n-1}\right)$

$128 \times\left(80 \times\left(\frac{1}{128}\right) \times\left(1-\frac{1}{128}\right)^{79}\right) \cong 43($ Coliso $) E(L, n, k)=L \times(1-p(L, n, 0)-p(L, n, 1))$

$128 \times(1-p(128,80,0)-p(128,80,1)) \Rightarrow 128 \times(1-0.534-0.336)$

$128 \times 0.13 \cong 16$

Exemplo A.0.7. Suponha que precise-se calcular a energia consumida do exemplo A.0.4 e quantas vezes $\left(N_{\text {protocol }}\right)$ uma bateria de $450 \mathrm{mAh}, 1.5 \mathrm{~V}$ pode ser utilizada para ler o conjunto de etiquetas. Temos então:

$$
\begin{gathered}
E=P \times T_{\text {total }}=2.25 \times 28=63 J \\
E=63 J \\
B=\left(\frac{(m A h \times V)}{1000}\right) \times 3600=\left(\frac{(450 \times 1.5)}{1000}\right) \times 3600=2430 J \\
N_{\text {protocol }}=\frac{B}{E_{\text {total }}}=\frac{2430}{63} \cong 38 \\
N_{\text {protocol }} \cong 38
\end{gathered}
$$




\section{Referências Bibliográficas}

Andrade e Goncalves (2013) Julio Andrade e Paulo Goncalves. Um Estimador Acurado para o Protocolo DFSA em Sistemas RFID. Em Proceedings of the SBRC 2013 (Brazilian Symposium on Computer Networks and Distributed Systems), páginas 325-338. Citado na pág. 37

Arjona et al. (2015) Laura Arjona, Hugo Landaluce, Asier Perallos, Pedro Lopez-Garcia e Nikola Cmiljanic. Analysis of rfid anti-collision protocols based on the standard epcglobal class-1 generation-2. Em European Wireless 2015; 21th European Wireless Conference; Proceedings of, páginas 1-6. Citado na pág. 29, 32

Baloch e Pendse (2013) Fariha Baloch e Ravi Pendse. Comparison of Transmission Control Protocols Based on EPC C1G2 Standard. Int. J. Com. Net. Tech, 1(1):83-94. Citado na pág. $85,87,89$

Bekkali et al. (2015) A. Bekkali, Sicheng Zou, A. Kadri, M. Crisp e R. Penty. Performance analysis of passive uhf rfid systems under cascaded fading channels and interference effects. Wireless Communications, IEEE Transactions on, 14(3):1421-1433. ISSN 1536-1276. doi: 10.1109/TWC.2014.2366142. Citado na pág. 56, 127, 132, 137

Bouras et al. (2013) Christos Bouras, Savvas Charalambides, Michalis Drakoulelis, Georgios Kioumourtzis e Kostas Stamos. A Tool for Automating Network Simulation and Processing Tracing Data Files. Simulation Modelling Practice and Theory, 30:90-110. ISSN 1569-190X. doi: \{10.1016/j.simpat.2012.09.001\}. Citado na pág. 23

Bueno-Delgado et al. (2013) M.V. Bueno-Delgado, R. Ferrero, F. Gandino, P. PavonMarino e M. Rebaudengo. A Geometric Distribution Reader Anti-Collision Protocol for RFID Dense Reader Environments. Automation Science and Engineering, IEEE Transactions on, 10(2):296-306. ISSN 1545-5955. doi: 10.1109/TASE.2012.2218101. Citado na pág. 144

Campbell (2011) Anita Campbell. Qr codes, barcodes and rfid: What is the difference ?, Fevereiro $2011 . \quad$ URL http://smallbiztrends.com/2011/02/qr-codes-barcodes-rfid-difference.html. Citado na pág. 2

Capetanakis (1979) J. Capetanakis. Tree algorithms for packet broadcast channels. Information Theory, IEEE Transactions on, 25(5):505-515. ISSN 0018-9448. doi: 10.1109/TIT.1979.1056093. Citado na pág. 18

Cerciello et al. (2014) E. Cerciello, G. Massei e L. Paura. Optimization of tag anti-collision algorithm for epc gen2 rfid. Em Euro Med Telco Conference (EMTC), 2014, páginas 1-6. doi: 10.1109/EMTC.2014.6996631. Citado na pág. 32, 127 
Chen (2015) W. Chen. Optimal frame length analysis and an efficient anticollision algorithm with early adjustment of frame length for rfid systems. Vehicular Technology, IEEE Transactions on, PP(99):1-1. ISSN 0018-9545. doi: 10.1109/TVT.2015.2441052. Citado na pág. Xv, 3, 35, 58, 62, 63, 127, 131, 133

Chen (2012) Wen-Tzu Chen. A New RFID Anti-Collision Algorithms for the EPCglobal UHF Class-1 Generation-2 standard. Em Proceedings of the 9th International Conference on Ubiquitous Intelligence $\&$ Computing and 9th International Conference on Autonomic \&6 Trusted Computing (UIC/ATC), páginas 811-815. IEEE COMPUTER SOC. ISBN 978-0-7695-4843-2. doi: $\{10.1109 /$ UIC-ATC.2012.73\}. Citado na pág. 87, 89

Chen (2014) Wen-Tzu Chen. A Feasible and Easy-to-Implement Anticollision Algorithm for the EPCglobal UHF Class-1 Generation-2 RFID Protocol. Automation Science and Engineering, IEEE Transactions on, 11(2):485-491. ISSN 1545-5955. doi: 10.1109/TASE. 2013.2257756. Citado na pág. vii, xv, 33, 34, 35, 36, 58, 68, 115, 120, 127, 132

Choi et al. (2012) Sunwoong Choi, Jaehyuk Choi e Joon Yoo. An efficient anti-collision protocol for tag identification in RFID systems with capture effect. Em Ubiquitous and Future Networks (ICUFN), 2012 Fourth International Conference on, páginas 482-483. doi: 10.1109/ICUFN.2012.6261756. Citado na pág. 25, 127

Cui e Zhao (2009) Yinghua Cui e Yuping Zhao. A modified q-parameter anti-collision scheme for rfid systems. Em Ultra Modern Telecommunications Workshops, 2009. ICUMT '09. International Conference on, páginas 1-4. doi: 10.1109/ICUMT.2009.5345419. Citado na pág. vii, xvi, $6,46,47,57,58,92,93,127$

Cui e Zhao (2010) Yinghua Cui e Yuping Zhao. A fast zero estimation scheme for RFID systems. Computer Communications, 33(11):1318 1324. ISSN 0140-3664. doi: http://dx.doi.org/10.1016/j.comcom.2010.03.012. URL http://www.sciencedirect.com/science/article/pii/S0140366410001210. Citado na pág. vii, xvi, 39, 58, 127

Das (2014) Raghu Das. Passive RFID grows by 1.12 billion tags in 2014 to 6.9 billion. http://goo.gl/cuQvnT, 2014. [Online; accessed 30-July-2015]. Citado na pág. 2

Das e Harrop (2014) Raghu Das e Peter Harrop. RFID Forecasts, Players and Opportunities 2014-2024. http://goo.gl/o9X0xB, 2014. [Online; accessed 30-July-2015]. Citado na pág. 2

Dobkin (2012) Daniel M. Dobkin. The RF in RFID: Passive UHF RFID in Practice. Newnes, Newton, MA, USA. ISBN 0750682094, 9780750682091. Citado na pág. 1, 3, 9, 14

Dong et al. (2008) Qunfeng Dong, Ashutosh Shukla, Vivek Shrivastava, Dheeraj Agrawal, Suman Banerjee e Koushik Kar. Load balancing in large-scale RFID systems. Computer Networks, 52(9):1782 - 1796. ISSN 1389-1286. doi: http://dx.doi.org/10.1016/j.comnet.2008.03.003. URL http://www.sciencedirect.com/science/article/pii/S138912860800090X. Citado na pág. 144

Eom e Lee (2010) Jun-Bong Eom e Tae-Jin Lee. Accurate tag estimation for dynamic framed-slotted aloha in rfid systems. Communications Letters, IEEE, 14(1):60-62. ISSN 1089-7798. doi: 10.1109/LCOMM.2010.01.091378. Citado na pág. 37, 50, 58, 81, 98, 100, 101, $106,121,127$ 
EPCglobal, GS1 Inc. (2015) EPCglobal, GS1 Inc. EPCTM Radio-Frequency Identity Protocols Class-1 Generation-2 UHF RFID Protocol for Communications at $860 \mathrm{MHz}-960 \mathrm{MHz}$ Version 2.0.1. Relatório técnico, EPCGlobal Inc. URL http://www.gs1.org/epcrfid/epc-rfid-uhf-air-interface-protocol/2-0-1. Citado na pág. vii, 23, $28,29,32,48,50,58,61,62,66,81,84,98,101,114,121,128,132,137$

Finkenzeller et al. (2010) K. Finkenzeller et al. RFID Handbook: Fundamentals and Applications in Contactless Smart Cards, Radio Frequency Identification and Near-field Communication. Wiley. Citado na pág. 1, 2, 3, 9, 10, 11, 12, 13, 14, 16, 17, 36, 56, 75

Floerkemeier e Sarma (2009) C. Floerkemeier e S. Sarma. Rfidsim: A physical and logical layer simulation engine for passive rfid. Automation Science and Engineering, IEEE Transactions on, 6(1):33-43. ISSN 1545-5955. doi: 10.1109/TASE.2008.2007929. Citado na pág. 23, 106, 107

Freitag e da Fonseca (2007) J. Freitag e N.L.S. da Fonseca. Wimax module for the ns-2 simulator. Em Personal, Indoor and Mobile Radio Communications, 200\%. PIMRC $200 \%$. IEEE 18th International Symposium on, páginas 1-6. doi: 10.1109/PIMRC.2007.4394155. Citado na pág. 24

Fritz et al. (2012) G. Fritz, V. Beroulle, O. Aktouf e D. Hely. Evaluation of a new rfid system performance monitoring approach. Em Design, Automation Test in Europe Conference Exhibition (DATE), 2012, páginas 1439-1442. doi: 10.1109/DATE.2012.6176591. Citado na pág. 23

Hakeem et al. (2014) Mohammed J Hakeem, Kaamran Raahemifar e Gul N Khan. Novel modulo based Aloha anti-collision algorithm for RFID systems. Em RFID (IEEE RFID), 2014 IEEE International Conference on, páginas 97-102. IEEE. Citado na pág. 4

Han et al. (2012) Heesun Han, Jongho Park e Tae-Jin Lee. RFID anti-collision protocol for monitoring system of tags in motion. Em Ubiquitous and Future Networks (ICUFN), 2012 Fourth International Conference on, páginas 318-321. doi: 10.1109/ICUFN.2012.6261720. Citado na pág. 143, 144

Hanada e Kudou (2013) Eisuke Hanada e Takato Kudou. Effective use of rfid in medicine. Em Medical Information and Communication Technology (ISMICT), 2013 7th International Symposium on, páginas 76-80. doi: 10.1109/ISMICT.2013.6521703. Citado na pág. xi, 13

He e Wang (2013) Yejun He e Xiaoye Wang. An ALOHA-based improved anti-collision algorithm for RFID systems. Wireless Communications, IEEE, 20(5):152-158. ISSN 1536-1284. doi: 10.1109/MWC.2013.6664486. Citado na pág. vii, xvi, 19, 52, 55, 58, 127

Huebner et al. (2012) Andreas Huebner, Christian Facchi e Helge Janicke. Rifidi toolkit: Virtuality for testing rfid. Em ICSNC 2012, The Seventh International Conference on Systems and Networks Communications, páginas 1-6. Citado na pág. 24

Kang et al. (2012) Donghoon Kang, Wei Wang, Byung Eon Park, Lin Xing e Sung Shin. Ns2 based wireless vehicular network performance study with high speed urban mobile relays. Em Communications and Networking in China (CHINACOM), 2012 7th International ICST Conference on, páginas 591-595. doi: 10.1109/ChinaCom.2012.6417552. Citado na pág. 24 
Khandelwal et al. (2006) Girish Khandelwal, A. Yener, Kyounghwan Lee e S. Serbetli. ASAP : A MAC Protocol for Dense and Time Constrained RFID Systems. Em Communications, 2006. ICC '06. IEEE International Conference on, volume 9, páginas 4028-4033. doi: 10.1109/ICC.2006.255711. Citado na pág. 55

Klair et al. (2009) Dheeraj Klair, Kwan-Wu Chin e Raad Raad. On the energy consumption of pure and slotted aloha based $\{$ RFID $\}$ anti-collision protocols. Computer Communications, 32(5):961 - 973. ISSN 0140-3664. doi: http://dx.doi.org/10.1016/j.comcom.2008.12. 019. URL http://www.sciencedirect.com/science/article/pii/S0140366408006555. Citado na pág. $3,38,86,119,120,121,131,147$

Klair et al. (2010) D.K. Klair, Kwan-Wu Chin e R. Raad. A Survey and Tutorial of RFID Anti-Collision Protocols. Communications Surveys Tutorials, IEEE, 12(3):400-421. ISSN 1553-877X. doi: 10.1109/SURV.2010.031810.00037. Citado na pág. xi, 2, 4, 5, 12, 13, 14, 16, $17,18,27,64$

Kodialam e Nandagopal (2006) Murali Kodialam e Thyaga Nandagopal. Fast and Reliable Estimation Schemes in RFID Systems. Em Proceedings of the 12th Annual International Conference on Mobile Computing and Networking, MobiCom '06, páginas 322-333, New York, NY, USA. ACM. ISBN 1-59593-286-0. doi: 10.1145/1161089.1161126. URL http://doi.acm.org/10.1145/1161089.1161126. Citado na pág. 33, 39

Lai e Hsiao (2010) Yuan-Cheng Lai e Ling-Yen Hsiao. General binary tree protocol for coping with the capture effect in rfid tag identification. Communications Letters, IEEE, 14(3):208-210. ISSN 1089-7798. doi: 10.1109/LCOMM.2010.03.092208. Citado na pág. 56

Lee et al. (2005) Su-Ryun Lee, Sung-Don Joo e Chae-Woo Lee. An enhanced dynamic framed slotted aloha algorithm for rfid tag identification. Em Mobile and Ubiquitous Systems: Networking and Services, 2005. MobiQuitous 2005. The Second Annual International Conference on, páginas 166-172. doi: 10.1109/MOBIQUITOUS.2005.13. Citado na pág. 53

Lessmann et al. (2008) J. Lessmann, P. Janacik, L. Lachev e D. Orfanus. Comparative study of wireless network simulators. Em Networking, 2008. ICN 2008. Seventh International Conference on, páginas 517-523. doi: 10.1109/ICN.2008.97. Citado na pág. 23

Li e Wang (2011) Bo Li e Junyu Wang. Efficient Anti-Collision Algorithm Utilizing the Capture Effect for ISO 18000-6C RFID Protocol. Communications Letters, IEEE, 15(3): 352-354. ISSN 1089-7798. doi: 10.1109/LCOMM.2011.011311.101332. Citado na pág. 25, 127

Li et al. (2009) Bo Li, Yuqing Yang e Junyu Wang. Anti-Collision Issue Analysis in Gen2 Protocol. Auto-ID Labs White Paper WP-HARDWARE-047, 3. URL http://www.autoidlabs.org/uploads/media/AUTOIDLABS-WP-HARDWARE-047.pdf. Citado na pág. 85

Li et al. (2010) Tao Li, S. Wu, Shigang Chen e M. Yang. Energy Efficient Algorithms for the RFID Estimation Problem. Em INFOCOM, 2010 Proceedings IEEE, páginas 1-9. Citado na pág. 62

Lin et al. (2015) Xiao-Hui Lin, Hui Wang, Yu-Kwong Kwok, Bin Chen, Mingjun Dai e Li Zhang. Exploiting the prefix information to enhance the performance of FSA-based RFID systems. Computer Communications, 56(0):108 - 
118. ISSN 0140-3664. doi: http://dx.doi.org/10.1016/j.comcom.2014.10.006. URL http://www.sciencedirect.com/science/article/pii/S0140366414003351. Citado na pág. xvi, 45,58

Maguire e Pappu (2009) Y. Maguire e R. Pappu. An optimal q-algorithm for the iso 18000-6c rfid protocol. Automation Science and Engineering, IEEE Transactions on, 6 (1):16-24. ISSN 1545-5955. doi: 10.1109/TASE.2008.2007266. Citado na pág. 56, 127

Mota (2015a) Rafael Perazzo Barbosa Mota. Simulator and Benchmark for RFID AntiCollision Evaluation. Em Proceedings of the Application of Information and Communication Technologies (AICT), 2015 IEEE 9th International Conference on, AICT '15, páginas 614-618, Rostov on Don, Rostov, Russia. IEEE. ISBN 978-1-4673-6856-8. doi: 10.1109/ICAICT.2015.7338634. Citado na pág. 7, 110

Mota (2015b) Rafael Perazzo Barbosa Mota. An Easy to Implement and Energy-saving Anti-Collision Algorithm for RFID Systems. Em Proceedings of the Application of Information and Communication Technologies (AICT), 2015 IEEE 9th International Conference on, AICT '15, páginas 367-372, Rostov on Don, Rostov, Russia. IEEE. ISBN 978-1-4673-6856-8. doi: 10.1109/ICAICT.2015.7338581. Citado na pág. 7, 147

Mota (2014) Rafael Perazzo Barbosa Mota. Student research abstract: Mechanisms to ensure quality of service for the internet of things. Em Proceedings of the 29th Annual ACM Symposium on Applied Computing, SAC '14, páginas 713-714, New York, NY, USA. ACM. ISBN 978-1-4503-2469-4. doi: 10.1145/2554850.2555189. URL http://doi.acm.org/10.1145/2554850.2555189. Citado na pág. 7

Mota e Batista (2013) Rafael Perazzo Barbosa Mota e Daniel Batista. Um Mecanismo para Garantia de QoS na "Internet das Coisas"com RFID. Em Proceedings of the SBRC 2013 (Brazilian Symposium on Computer Networks and Distributed Systems), páginas 297-310. Citado na pág. 6, 80

Mota e Batista (2014) Rafael Perazzo Barbosa Mota e Daniel M. Batista. A Dynamic Frame Slotted ALOHA Anti-collision Algorithm for the Internet of Things. Em Proceedings of the 29th Annual ACM Symposium on Applied Computing, páginas 686-691. ACM. ISBN 978-1-4503-2469-4. doi: 10.1145/2554850.2555003. Citado na pág. 7, 58, 61

Namboodiri et al. (2012) Vinod Namboodiri, Maheesha DeSilva, Kavindya Deegala e Suresh Ramamoorthy. An extensive study of slotted alohabased $\{$ RFID $\}$ anti-collision protocols. Computer Communications, 35(16): 1955 - 1966. ISSN 0140-3664. doi: 10.1016/j.comcom.2012.05.015. URL http://www.sciencedirect.com/science/article/pii/S0140366412001776. Citado na pág. $2,62,106$

Namboodiri et al. (2012) Vinod Namboodiri, Maheesha DeSilva, Kavindya Deegala e Suresh Ramamoorthy. An Extensive Study of Slotted Aloha-based RFID Anti-collision Protocols. Computer Communications, 35(16):1955-1966. ISSN 0140-3664. doi: $\{10.1016 /$ j.comcom.2012.05.015\}. Citado na pág. 62, 85, 86, 106, 119, 120, 131, 144, 145

Natali et al. (2010) C.-F. Natali, N.F. Vaidya e V.K.Y. Wu. Expanding Horizon and Capture Effect in RFID Singulation. Em Global Telecommunications Conference (GLOBECOM 2010), 2010 IEEE, páginas 1-5. doi: 10.1109/GLOCOM.2010.5683908. Citado na pág. 25, 127 
Ou e He (2013) Chia-Ho Ou e Wei-Lun He. Path planning algorithm for mobile anchorbased localization in wireless sensor networks. Sensors Journal, IEEE, 13(2):466-475. ISSN 1530-437X. doi: 10.1109/JSEN.2012.2218100. Citado na pág. 24

Perazzo Barbosa Mota e Batista (2013) R. Perazzo Barbosa Mota e D.M. Batista. An ns-2 module for simulating passive rfid systems. Em High Performance Computing and Communications 2013 IEEE International Conference on Embedded and Ubiquitous Computing (HPCC-EUC), 2013 IEEE 10th International Conference on, páginas 22632270. doi: 10.1109/HPCC.and.EUC.2013.325. Citado na pág. 6, 90

Perazzo Barbosa Mota e Batista (2013) Rafael Perazzo Barbosa Mota e Daniel M. Batista. A RFID QoS mechanism for IoT tracking applications. Em 8th International Symposium on Wireless and Pervasive Computing 2013 (ISWPC 2013), Taipei, Taiwan, Taiwan. Citado na pág. 6, 80

Perera et al. (2015) C. Perera, C.H. Liu e S. Jayawardena. The Emerging Internet of Things Marketplace From an Industrial Perspective: A Survey. Emerging Topics in Computing, IEEE Transactions on, PP(99):1-1. ISSN 2168-6750. doi: 10.1109/TETC.2015.2390034. Citado na pág. 144

Prodanoff (2010) Zornitza Genova Prodanoff. Optimal frame size analysis for framed slotted ALOHA based RFID networks. Computer Communications, 33(5):648 - 653. ISSN 0140-3664. doi: http://dx.doi.org/10.1016/j.comcom.2009.11.007. URL http://www.sciencedirect.com/science/article/pii/S0140366409002965. Citado na pág. 25

R Core Team (2013) R Core Team. R: A Language and Environment for Statistical Computing. R Foundation for Statistical Computing, Vienna, Austria, 2013. URL http://www.R-project.org/. Citado na pág. 132

Sanchez e Smith (1999) J. Sanchez e D.R. Smith. Capture effect in rician fading channels with application to slotted aloha. Em Global Telecommunications Conference, 1999. GLOBECOM '99, volume 5, páginas 2390-2394 vol.5. doi: 10.1109/GLOCOM.1999.831730. Citado na pág. 56, 132, 137

Sanchez M e Ramos R (2012) Leonardo D. Sanchez M e Victor M. Ramos R. Adding Randomness to the EPC Class1 Gen2 Standard for RFID Networks. Em Proceedings of the 23rd IEEE PIMRC, páginas 609-614. ISBN 978-1-4673-2569-1. Citado na pág. 87, 89

Schoute (1983) F.C. Schoute. Dynamic frame length aloha. Communications, IEEE Transactions on, 31(4):565-568. ISSN 0090-6778. doi: 10.1109/TCOM.1983.1095854. Citado na pág. 34, 35, 36, 40, 43, 50, 57, 58, 81, 98, 100, 101, 106, 107, 113, 114, 121, 127, 140, 148

Schwartz (1987) Mischa Schwartz. Telecommunication networks: protocols, modeling and analysis, volume 7. Addison-Wesley Reading. Citado na pág. 18

Shakiba et al. (2014) Masoud Shakiba, Mandeep Jit Singh, Elankovan Sundararajan, Azam Zavvari e Mohammad Tariqul Islam. Extending Birthday Paradox Theory to Estimate the Number of Tags in RFID Systems. PLoS ONE, 9(4):e95425. doi: 10.1371/journal. pone.0095425. Citado na pág. xvi, 41, 58, 63, 120, 121, 125, 133

Shih et al. (2006) Dong-Her Shih, Po-Ling Sun, David C. Yen e Shi-Ming Huang. Taxonomy and Survey of RFID Anti-Collision Protocols. Computer Communications, 29(11):2150 2166. ISSN 0140-3664. doi: http://dx.doi.org/10.1016/j.comcom.2005.12.011. Citado na pág. 5,18 
Shin e Kim (2009) Woo Jin Shin e Jeong Geun Kim. A capture-aware access control method for enhanced RFID anti-collision performance. Communications Letters, IEEE, 13(5):354-356. ISSN 1089-7798. doi: 10.1109/LCOMM.2009.081970. Citado na pág. 25

Song et al. (2013) Y. Song, B. Wang, Z. Shi, K. Pattipati e S. Gupta. Distributed algorithms for energy-efficient even self-deployment in mobile sensor networks. Mobile Computing, IEEE Transactions on, PP(99):1-1. ISSN 1536-1233. doi: 10.1109/TMC.2013.46. Citado na pág. 24

Spiegel et al. (2002) Murray Spiegel, John Schiller e Alu Srinivasan. Schaum's Easy Outline of Probability and Statistics. McGraw Hill Professional. Citado na pág. 20, 115

Swedberg (2015) Claire Swedberg. RFID Market for Retailers Forecast to Grow 39\% Annually. http://www.rfidjournal.com/articles/view?13104, 2015. [Online; accessed 30July-2015]. Citado na pág. 2

Tanenbaum (2010) Andrew Tanenbaum. Computer Networks. Prentice Hall Professional Technical Reference, 5th ed. ISBN 0130661023. Citado na pág. 15, 28

Tong et al. (2012) Qiaoling Tong, Qiao Zhang, Run Min e Xuecheng Zou. Bayesian estimation in dynamic framed slotted ALOHA algorithm for RFID system. Computers and Mathematics with Applications, 64(5):1179 - 1186. ISSN 0898-1221. doi: http://dx.doi.org/10.1016/j.camwa.2012.03.060. URL http://www.sciencedirect.com/science/article/pii/S0898122112002659._Advanced Technologies in Computer, Consumer and Control. Citado na pág. vii, xvi, 40, 42, 58

Ullah et al. (2012) S. Ullah, W. Alsalih, A. Alsehaim e N. Alsadhan. A Review of Tags Anti-collision and Localization Protocols in RFID Networks. Journal of Medical Systems, 36(6):4037-4050. ISSN 0148-5598. doi: 10.1007/s10916-012-9876-5. URL http://dx.doi.org/10.1007/s10916-012-9876-5. Citado na pág. 5, 18

Verma et al. (2013) Suraj Verma, Prashant Pillai e Yim-Fun Hu. Performance evaluation of alternative network architectures for sensor-satellite integrated networks. Em Advanced Information Networking and Applications Workshops (WAINA), 2013 27th International Conference on, páginas 120-125. doi: 10.1109/WAINA.2013.56. Citado na pág. 24

Violino (2005) Bob Violino. The history of rfid technology, Janeiro 2005. URL http://www.rfidjournal.com/articles/view?1338. Citado na pág. 10

Vogt (2002) Harald Vogt. Efficient object identification with passive rfid tags. Em Proceedings of the First International Conference on Pervasive Computing, Pervasive '02, páginas 98-113, London, UK, UK. Springer-Verlag. ISBN 3-540-44060-7. URL http://dl.acm.org/citation.cfm?id=646867.706691. Citado na pág. 37, 40, 42, 43, 101, 107, 121

Waktola (2015) Enu Waktola. Internet of Things Promises Huge RFID Growth. https://goo.gl/CaCkfx, 2015. [Online; accessed 30-July-2015]. Citado na pág. 2

Wang et al. (2009) Chonggang Wang, Mahmoud Daneshmand e Kazem Sohraby. Optimization of tag reading performance in generation-2 RFID protocol. Computer Communications, 32(11):1346 - 1352. ISSN 0140-3664. doi: http://dx.doi.org/10.1016/j.comcom. 2009.02.004. URL http://www.sciencedirect.com/science/article/pii/S0140366409000541. Special Issue of Computer Communications on Heterogeneous Networking for Quality, Reliability, Security, and Robustness - Part I. Citado na pág. xv, 33, 34, 58 
Wang et al. (2014) Hui Wang, Shengliang Xiao, Feiyu Lin, Tianchu Yang e LaurenceT. Yang. Group improved enhanced dynamic frame slotted ALOHA anti-collision algorithm. The Journal of Supercomputing, 69(3):1235-1253. ISSN 0920-8542. doi: 10.1007/s11227-014-1189-7. URL http://dx.doi.org/10.1007/s11227-014-1189-7. Citado na pág. vii, xvi, 53, 54, 58, 118

Wang et al. (2012) Shuai Wang, Weijun Hong, Liang Yin e ShuFang Li. A novel fast tag estimate method for dynamic frame length aloha anti-collision algorithms in rfid system. Em Vehicular Technology Conference (VTC Fall), 2012 IEEE, páginas 1-5. doi: 10.1109/ VTCFall.2012.6399144. Citado na pág. 9

WANG et al. (2013) Shuai WANG, Wei jun HONG, Biao PENG e Shu fang LI. Novel error resilient tag estimation for RFID system in interference scenarios. The Journal of China Universities of Posts and Telecommunications, 20(4):116 - 121. ISSN 1005-8885. doi: http://dx.doi.org/10.1016/S1005-8885(13)60079-5. URL http://www.sciencedirect.com/science/article/pii/S1005888513600795. Citado na pág. 145

Wang et al. (2010) Wei Wang, H. Sharif, M. Hempel, Ting Zhou, P. Mahasukhon e Tao Ma. Implementation and performance evaluation of a complete, accurate, versatile and realistic simulation model for mobile wimax in ns-2. Em Communications (ICC), 2010 IEEE International Conference on, páginas 1-5. doi: 10.1109/ICC.2010.5502328. Citado na pág. 24

Wang et al. (2007) Zhongxiang Wang, Dan Liu, Xiaofang Zhou, Xi Tan, Junyu Wang e Hao Min. Anti-Collision Scheme Analysis of RFID System. Auto-ID Labs White Paper. URL http://www.autoidlabs.org/uploads/media/AUTOIDLABS-WP-HARDWARE-045.pdf. Citado na pág. 85

Want (2006) R. Want. An introduction to rfid technology. Pervasive Computing, IEEE, 5 (1):25-33. ISSN 1536-1268. doi: 10.1109/MPRV.2006.2. Citado na pág. 1

Weingartner et al. (2009) E. Weingartner, H. vom Lehn e K. Wehrle. A performance comparison of recent network simulators. Em Communications, 2009. ICC '09. IEEE International Conference on, páginas 1-5. doi: 10.1109/ICC.2009.5198657. Citado na pág. 23, 24

Welbourne et al. (2009) E. Welbourne, L. Battle, G. Cole, K. Gould, K. Rector, S. Raymer, M. Balazinska e G. Borriello. Building the Internet of Things Using RFID: The RFID Ecosystem Experience. Internet Computing, IEEE, 13(3):48 -55. ISSN 1089-7801. doi: 10.1109/MIC.2009.52. Citado na pág. 15, 71, 72

Wu e Zeng (2010) Haifeng Wu e Yu Zeng. Bayesian Tag Estimate and Optimal Frame Length for Anti-Collision Aloha RFID System. Automation Science and Engineering, IEEE Transactions on, 7(4):963-969. ISSN 1545-5955. doi: 10.1109/TASE.2010.2042957. Citado na pág. $58,121,125$

Wu e Zeng (2015) Haifeng Wu e Yu Zeng. Passive RFID Tag Anticollision Algorithm for Capture Effect. Sensors Journal, IEEE, 15(1):218-226. ISSN 1530-437X. Citado na pág. 25, 26, 56, 67, 127, 132, 135, 137, 140

Wu et al. (2013) Haifeng Wu, Yu Zeng, Jihua Feng e Yu Gu. Binary tree slotted aloha for passive rfid tag anticollision. Parallel and Distributed Systems, IEEE Transactions on, 24 
(1):19-31. ISSN 1045-9219. doi: 10.1109/TPDS.2012.120. Citado na pág. vii, xiii, xvi, 3, 6, $10,16,21,27,30,48,49,50,51,56,58,85,86,87,88,89,91,101,102,106,115,118,119$, $126,127,140$

Wu et al. (2010) Miao Wu, Ting-Jie Lu, Fei-Yang Ling, Jing Sun e Hui-Ying Du. Research on the architecture of internet of things. Em Advanced Computer Theory and Engineering (ICACTE), 2010 3rd International Conference on, volume 5, páginas V5-484-V5-487. doi: 10.1109/ICACTE.2010.5579493. Citado na pág. 63

Xu e Chen (2015) Ying Xu e Yifan Chen. An improved dynamic framed slotted aloha anti-collision algorithm based on estimation method for rfid systems. Em RFID (RFID), 2015 IEEE International Conference on, páginas 1-8. doi: 10.1109/RFID.2015.7113066. Citado na pág. xvi, 55, 57, 58

Yao et al. (2014) Junmei Yao, Tao Xiong e Wei Lou. Beyond the limit: A fast tag identification protocol for RFID systems. Pervasive and Mobile Computing, (-):-. ISSN 1574-1192. doi: http://dx.doi.org/10.1016/j.pmcj.2014.10.003. URL http://www.sciencedirect.com/science/article/pii/S1574119214001667. Citado na pág. vii, xvi, $43,44,58$

Zhang et al. (2013) Linchao Zhang, Renato Ferrero, Filippo Gandino e Maurizio Rebaudengo. Evaluation of single and additive interference models for RFID collisions. Mathematical and Computer Modelling, 58(5?6):1236 - 1248. ISSN 0895-7177. doi: http://dx.doi.org/10.1016/j.mcm.2013.01.011. URL http://www.sciencedirect.com/science/article/pii/S0895717713000307. The Measurement of Undesirable Outputs: Models Development and Empirical Analyses and Advances in mobile, ubiquitous and cognitive computing. Citado na pág. 145

Zhang et al. (2012a) Tiancheng Zhang, Yifang Yin, Dejun Yue, Qian Ma e Ge Yu. A simulation platform for rfid application deployment supporting multiple scenarios. Em Computational Intelligence and Security (CIS), 2012 Eighth International Conference on, páginas 563-567. doi: 10.1109/CIS.2012.132. Citado na pág. 23

Zhang et al. (2012b) Yanjun Zhang, Siye Wang, Zhenyu Liu, Wenbiao Zhou e D. Liu. Performance analysis of wireless sensor network based on ns-2. Em Systems and Informatics (ICSAI), 2012 International Conference on, páginas 1445-1448. doi: 10.1109/ICSAI. 2012.6223308. Citado na pág. 24

Zhou et al. (2013) Lei Zhou, Ying Zhu, Ai Chen e Cong Liu. A simulation platform for zigbee-umts hybrid networks. Communications Letters, IEEE, 17(2):293-296. ISSN 1089-7798. doi: 10.1109/LCOMM.2012.121912.121974. Citado na pág. 24

Zhu e Yum (2011) Lei Zhu e T.-S.P. Yum. A critical survey and analysis of RFID anticollision mechanisms. Communications Magazine, IEEE, 49(5):214-221. ISSN 0163-6804. doi: 10.1109/MCOM.2011.5762820. Citado na pág. 18

Zhu e Yum (2010) Lei Zhu e T.P. Yum. Optimal Framed Aloha Based Anti-Collision Algorithms for RFID Systems. Communications, IEEE Transactions on, 58(12):35833592. ISSN 0090-6778. doi: 10.1109/TCOMM.2011.102910.090390. Citado na pág. xvi, 5, 38, 58 
Zorzi (1997) M. Zorzi. Capture probabilities in random-access mobile communications in the presence of rician fading. Vehicular Technology, IEEE Transactions on, 46(1):96-101. ISSN 0018-9545. doi: 10.1109/25.554741. Citado na pág. 56, 132, 137 